\title{
An ethnographic study of factors associated with poorly controlled Type-2 diabetes and its management among patients accessing primary care services in Jordan
}

\author{
Al-Sahouri, Amer M.
}

How to cite:

Al-Sahouri, Amer M. (2019) An ethnographic study of factors associated with poorly controlled Type-2 diabetes and its management among patients accessing primary care services in Jordan. Doctoral thesis, Swansea University. http://cronfa.swan.ac.uk/Record/cronfa50314

Use policy:

This item is brought to you by Swansea University. Any person downloading material is agreeing to abide by the terms of the repository licence: copies of full text items may be used or reproduced in any format or medium, without prior permission for personal research or study, educational or non-commercial purposes only. The copyright for any work remains with the original author unless otherwise specified. The full-text must not be sold in any format or medium without the formal permission of the copyright holder. Permission for multiple reproductions should be obtained from the original author.

Authors are personally responsible for adhering to copyright and publisher restrictions when uploading content to the repository.

Please link to the metadata record in the Swansea University repository, Cronfa (link given in the citation reference above.) 


\section{Title of thesis}

An ethnographic study of factors associated with poorly controlled

Type-2 diabetes and its management among patients accessing primary care services in Jordan

Amer Moh'd Ahmad Al-Sahouri

Submitted to Swansea University in fulfilment of the requirements for the Degree of Doctor of Philosophy

Swansea University

2019 


\begin{abstract}
Poorly controlled Type-2 diabetes is considered a major public health problem and associated with adverse outcomes in Jordan. However, there is a shortfall in qualitative research relating to diabetes. There are no published studies that have sought to explore the meaning behind people's health behaviours and the influence of socio-cultural norms on the management of Type- 2 diabetes in Jordan. This study aimed to explore the reasons why glycaemic control was poor among patients with Type- 2 diabetes who used primary care services provided by a selected health care comprehensive centre in Jordan.
\end{abstract}

An ethnographic approach was used encompassing a mixed methods data collection strategy which included: 80 hours of participant observation, semi-structured interviews with 10 health care workers and 6 family carers, 8 focus groups with 38 patients with poorly controlled Type2 diabetes and documentary review of health promotion resources.

The study findings suggested that influences on patients' diabetes management occurred at multiple levels rather than one level. Intrapersonal factors included the difficulty in maintaining adherence to the management plan, fatalistic attitudes and misconceptions related to diabetes. Sociocultural factors included: eating habits, the stigma of the disease, social embarrassment with physical activity and medication and seeking complementary therapies. The health care system factors included: medication insecurity, health insurance policies and unavailability of laboratory tests. Staff shortages, patient-staff communication and being task-focused were challenges relating to health care workers. Continuity of care and health education were suggested as facilitators and using the mass media, schools and religion were also proposed to promote the health of the public.

Effective health promotion interventions and health education need to be tailored to meet the individual, social and cultural needs of patients. A patient-centred, and holistic approach is recommended. Interventions at multiple levels from the individual to the policy level are required in order to address the complexity of factors affecting patients' adherence to their diabetes management plan in Jordan. This study has particular relevance for primary care clinics in Jordan but may also more generally contribute towards an understanding of poor glycaemic control in patients with Type- 2 diabetes in countries with similar socio-cultural norms. 


\section{DECLARATION}

This work has not previously been accepted in substance for any degree and is not being concurrently submitted in candidature for any degree.

Signed (candidate)

Date

\section{STATEMENT 1}

This thesis is the result of my own investigations, except where otherwise stated. Where correction services have been used, the extent and nature of the correction is clearly marked in a footnote(s).

Other sources are acknowledged by footnotes giving explicit references. A bibliography is appended.

Signed (candidate)

Date

\section{STATEMENT 2}

I hereby give consent for my thesis, if accepted, to be available for photocopying and for inter-library loan, and for the title and summary to be made available to outside organisations.

Signed (candidate)

Date 
NB: Candidates on whose behalf a bar on access has been approved by the University (see Note 7), should use the following version of Statement 2:

I hereby give consent for my thesis, if accepted, to be available for photocopying and for inter-library loans after expiry of a bar on access approved by the Swansea University.

Signed (candidate)

Date 


\section{Contents Page}

Abstract.

Declaration.

.2

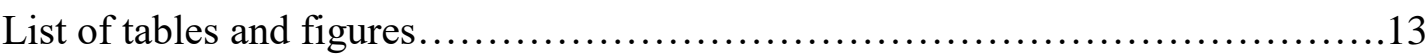

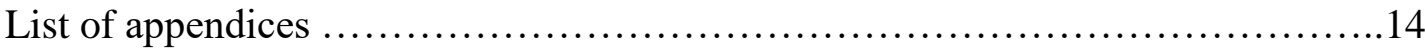

Abbreviations.............................................................. 16

Acknowledgement....................................................... 17

\section{CHAPTER ONE}

INTRODUCTION............................................................18-31

1.1 Introduction............................................................18

1.2 Introduction to the research topic........................................18

1.3 Aim and objectives of the study.........................................20

1.4 Overview of the health care system in Jordan............................21

1.5 Location of study setting..............................................24

1.5.1 The patient's journey through the centre ............................27

1.6 The layout of the thesis................................................30 


\section{CHAPTER TWO}

LITERATURE REVIEW ...................................................32-70

2.1 Introduction...............................................................32

2.1.2 Literature search strategy............................................. 32

2.2 The extent of Type-2 diabetes and its complications in Jordan.............36

2.3 Diabetes self-management behaviours.................................43

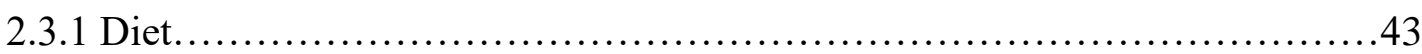

2.3.2 Physical activity................................................. 47

2.3.3 Adherence to medications...........................................50

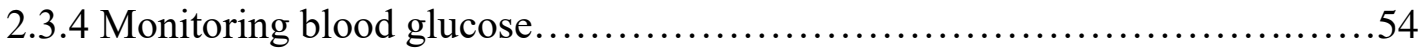

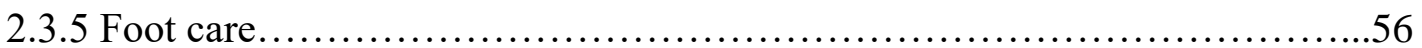

2.4 Attitudes, knowledge and perceptions of patients with Type-2 diabetes regarding diabetes......................................................................59

2.5 Complementary medicine and diabetes...................................64

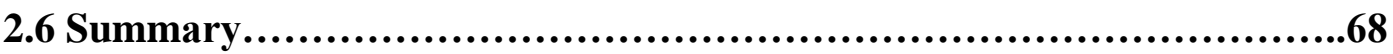

CHAPTER THREE

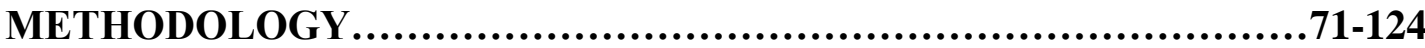

3.1 Introduction.............................................................71

3.1.1 Aim and objectives of the study....................................... 71 


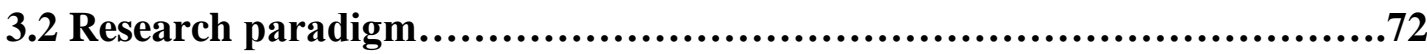

3.2.1 Ontological and epistemological frameworks of this study....................74

3.3 Qualitative research approach.............................................75

3.3.1 Ethnographic design............................................... 78

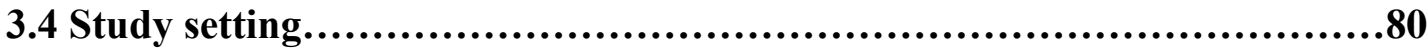

3.5 Sampling method.....................................................80

3.5.1 Sampling for participant observation.................................. 82

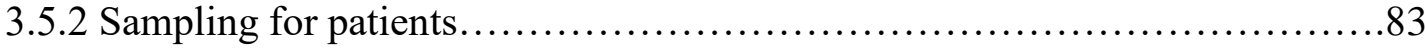

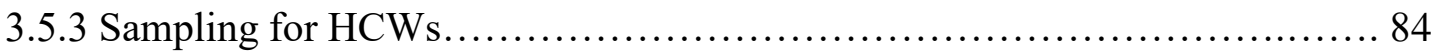

3.5.4 Sampling for Family carers........................................... 84

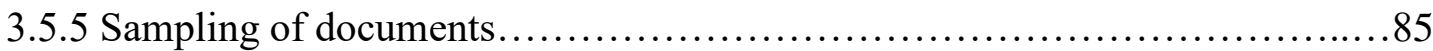

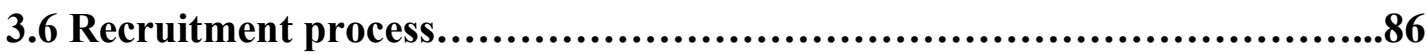

3.6.1 Initial approach.................................................... 86

3.6.2 Recruitment of samples........................................... 86

3.7 Methods of data collection...............................................88

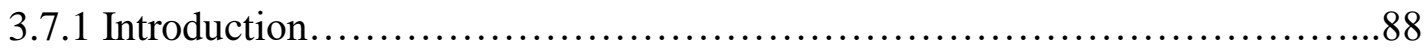

3.7.2 Participant observation.......................................... 88

3.7.2.1 Roles of the researcher during participant observations...................89

3.7.3 Focus groups.................................................92

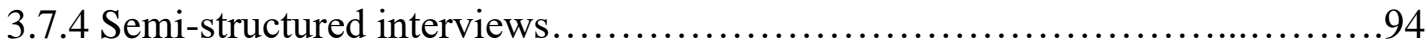


3.8 Ethical considerations................................................. 96

3.8.1 Informed consent...............................................97

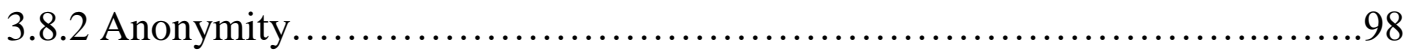

3.8.3 Confidentiality ................................................ 98

3.8.4 Harm to participants...............................................99

3.8.5 Ethical approval............................................... 100

3.9 Data handling and data analysis......................................100

3.9.1 Data handling ................................................... 100

3.9.2 Data analysis................................................. 101

3.10 Methodological rigour.................................................103

3.10.1 Lincoln and Guba's evaluation criteria (1985).......................... 103

3.10.2 Hammersley's evaluation criteria (1992)................................107

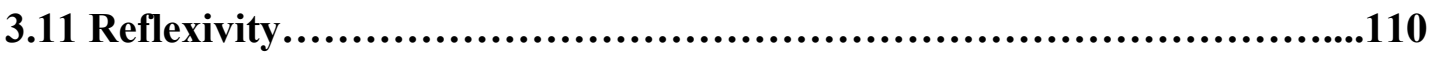

3.12 Overview of Theories...............................................113

3.12.1 Socio-ecological model (SEM) ................................ 113

3.12.2 Medical model of health care ...................................... 118

3.12.3 Health belief model (HBM) ...................................... 120

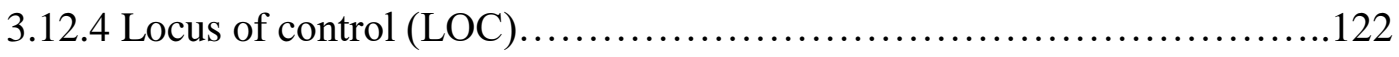

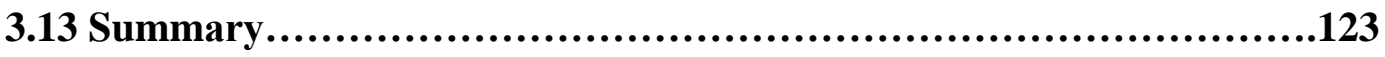




\section{CHAPTER FOUR}

INTRODUCTION TO RESULTS.....................................125-129

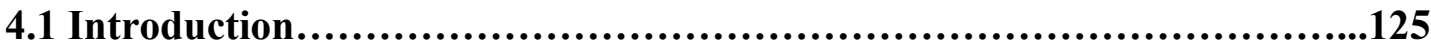

4.2 Characteristics of the sample............................................125

4.3 Main Themes of the Study.......................................................129

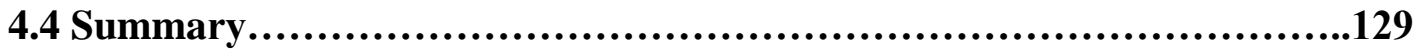

CHAPTER FIVE

ATTITUDES, KNOWLEDGE AND PERCEPTIONS OF PATIENTS REGARDING

TYPE-2 DIABETES.................................................130-165

5.1 Introduction............................................................130

5.2 Reasons for developing diabetes from the patients' perspectives...........130

5.2.1 Whatever happens, happens........................................... 131

5.2.2 Proactive screening for diabetes.....................................136

5.2.3 The reaction to classic symptoms of diabetes.......................... 138

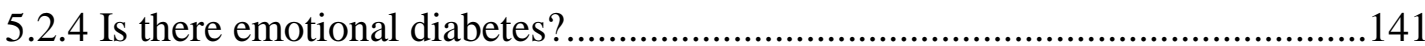

5.3 Patients' knowledge of Type-2 diabetes and its complications..............145

5.3.1 Patients' knowledge of Type-2 diabetes................................. 145

5.3.2 Patients' perception of diabetes complications.......................... 148 
5.4 Participants' perceptions of insulin and self-monitoring blood glucose....152

5.5 Patients' misconceptions of diabetes and its management....................156

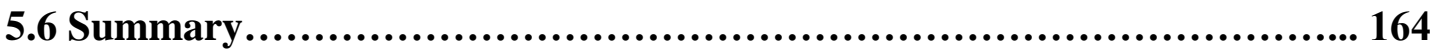

\section{CHAPTER SIX}

INFLUENCE OF THE JORDANIAN SOCIO-CULTURAL CONTEXT ON PATIENTS' DIABETES MANAGEMENT PLAN...............................166-196

6.1 Introduction..........................................................................166

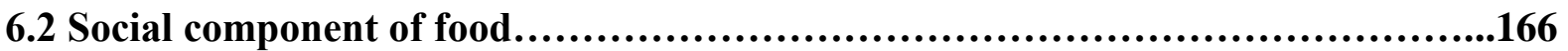

6.2.1 The role of food in the Jordanian socio-cultural context............................ 166

6.2.2 Emotional component of food 'forbidden phenomenon..........................175

6.3 Social factors influencing physical activity ....................................180

6.4 Complementary therapies..........................................................183

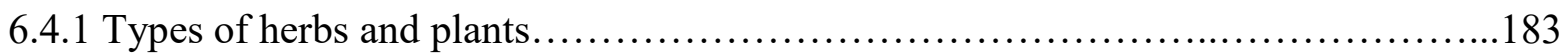

6.4.2 Reasons for seeking complementary medicine............................... 188

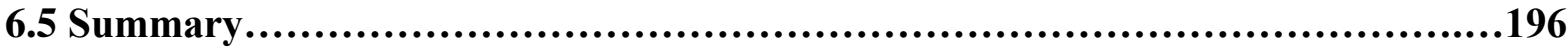

\section{CHAPTER SEVEN}

CHALLENGES TO GOOD GLYCAEMIC CONTROL RELATED TO THE HEALTH 
7.2.1 Impact of health insurance.

\section{CHAPTER EIGHT}

Facilitators to good glycaemic control levels.

8.2.1.1 Overview of the previous health education programme at the comprehensive health care centre. .229 
8.2.3 Sources of health information.

8.3 Facilitators at the health care system (HCS) level.

\section{CHAPTER NINE}

CONCLUSION

9.1 Introduction .267

9.2 Summary of main findings.............................................268

9.3 Limitations of this study $\ldots \ldots \ldots \ldots \ldots \ldots \ldots \ldots \ldots \ldots \ldots \ldots \ldots \ldots \ldots \ldots \ldots \ldots \ldots \ldots \ldots . . . . \ldots 271$

9.4 Relevance of the study findings............................................ 273

9.4.1 Relevance and recommendations for patients..........................273

9.4.2 Relevance and recommendations for health care workers and managers.....274

9.4.3 Relevance and recommendations for the wider community................277

9.4.4 Relevance and recommendations for policy makers. 
Appendices............................................................282-327

References................................................................328-353 


\section{List of Tables}

Table 1. The number of HCWs on duty per day in the studied comprehensive health care centre. 25

Table 2. The English, local and scientific names of herbs that were used by patients with diabetes in Jordan in the literature .66

Table 3. Sociodemographic and relevant characteristics of patients with Type-2 diabetes $(n=38)$ 125

Table 4. All the plants and herbs that have been reportedly used by patients with diabetes in my study compared to the previous studies in Jordan .187

\section{List of Figures}

Figure 1. Map of Jordan .24

Figure 2. The process of movement of the patient between different departments within the centre for receiving care

Figure 3. The barriers that patients may experience during their visit to the centre...29

Figure 4. PRISMA literature search flowchart. .35

Figure 5. Roles of researcher during participant observations .91

Figure 6. Levels of influences in the socio-ecological health model with examples from my study findings.

Figure 7. Pictures and the English translation of the dialogue between Abu Sami and his family taken from the educational show 'Abu Sami' from the previous educational programme in the centre. 


\section{List of appendices}

Appendix 1. CHHS Research Ethics Committee approval letter.................282

Appendix 2. The Ministry of Health ethical approval letter.....................283

Appendix 3. The Ministry of Health covering letter...........................286

Appendix 4. Invitation letter to group discussion participants.................287

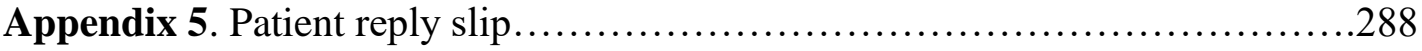

Appendix 6. Participant information sheet (group discussion).................289

Appendix 7. Participant information sheet (Health care workers)................292

Appendix 8. Participant information sheet (Family carers) $\ldots \ldots \ldots \ldots \ldots \ldots \ldots \ldots \ldots \ldots 295$

Appendix 9. Participant consent form....................................298

Appendix 10. Group discussion topic guide...............................300

Appendix 11.Semi structured interview topic guide HCWs................... 303

Appendix 12. Semi structured interview topic guide (Family carers) ........... 306

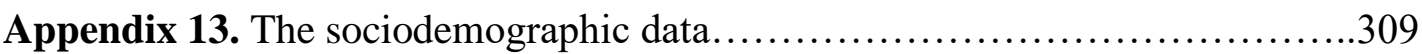

Appendix 14. Debrief sheet.......................................... 311

Appendix 15. Participant observation poster............................... 312

Appendix 16. General information of the focus groups.......................313

Appendix 17. General Information of Health Care Workers who were interviewed in this study. 315

Appendix 18. General Information of Family Carers who were interviewed in this study.... 316 
Appendix 19. All of the keywords used in the search process................... 317

Appendix 20. Example of thematic development...........................3 318

Appendix 21. Description of studies included in the literature review..............321

Appendix 22. Publications and conference presentations from this study...........327 
Abbreviations

-ADA: American Diabetes Association

-BMI: Body Mass Index

-FG: Focus group

-HCWs: Health care workers

-IDF: International Diabetes Federation

-HBM: Health Belief Model

-MOH: Ministry of Health

-NCDEG: The National Centre for Diabetes, Endocrinology and Genetics

-NHS: National Health Service

-PRISMA: Preferred Reporting Items for Systematic Reviews and Meta-Analyses

-SMBG: Self-monitoring blood glucose

-SEM: Socio-ecological model

-TRHAS: The Royal Health Awareness Society

-T2DM: Type-2 diabetes mellitus

-UAE: United Arab Emiratis

-UK: United Kingdom

-UNRWA: United Nations Relief and Works Agency for Palestine refugees

-USA: United States of America

-WHO: World Health Organisation 


\section{Acknowledgement}

I would like to thank my supportive supervisor Professor Joy Merrell who guided me through all the stages of my research and for her hard work and valuable feedback. Special thanks also to Doctor Sherrill Snelgrove for offering supervision, granting me the time, and sustained interest throughout my PhD journey.

Special thanks to my wife, Faith, for support and encouragement that I have received during my $\mathrm{PhD}$.

I would like to thank all my family members, particularly my sister Asma and her husband Moutaz for their care and support.

Finally, special thanks to all study participants, particularly patients with poorly controlled Type2 diabetes who generously gave their time and efforts to take part in my research. 


\section{CHAPTER ONE}

\section{INTRODUCTION}

\subsection{Introduction}

This study sought to gain a better understanding of the factors associated with the management of Type-2 diabetes mellitus (T2DM) among patients within the socio-cultural context of Jordan. This chapter presents an introduction to the research study and provides background information. This includes an overview of the health care system in Jordan, aims and objectives of this study, the location of the study setting and finally, the organisation of the thesis. First, an introduction to the research study is presented.

\subsection{Introduction to the research topic}

Type-2 diabetes is a chronic condition and the most prevalent type of diabetes (IDF, 2017). It occurs when an inadequate amount of insulin is produced by the body, or the body cells do not react adequately to insulin (recognised as insulin resistance), resulting in high blood glucose levels (Hillson, 2015). Abnormalities in blood glucose levels in T2DM influence multiple organs such as the liver, skeletal muscles, adipose tissue and pancreatic beta cells (Unger, 2012). Types of diabetes include Type- 2 diabetes, Type- 1 diabetes and gestational diabetes (Hillson, 2015). T2DM is diagnosed by two results of glycated haemoglobin (HbA1c) levels of $6.5 \%$ or more (ADA, 2017).

Globally, there are 425 million people who have diabetes, and this number is expected to increase to 629 million by 2045 (IDF, 2017). $80 \%$ of patients with diabetes live in low- and middle- income countries. In 2017, four million people died from diabetes, and it is estimated that the financial burden of diabetes worldwide in 2017 was 727 Billion US dollars (IDF, 2017). Globally, it is estimated that 212.4 million people are unaware of their disease, and are therefore considered as undiagnosed cases, with $84.5 \%$ of them are in low- and middle- income countries (IDF, 2017).

Diabetes mellitus is a significant health problem in the Middle East region. The prevalence rate of diabetes is expected to increase from 39 million people in 2017 to 82 million people in 2045 , and it is considered the second highest prevalence among the world regions after North 
America and the Caribbean (IDF, 2017). In 2016, 11.8\% of the population in Jordan is reported to have diabetes (IDF, 2017).

According to the American Diabetes Association (2017), glycaemic control levels are essential to monitor diabetes management. Good glycaemic control levels have been shown to decrease rates of diabetes microvascular complications such as retinopathy, nephropathy and neuropathy (Hillson, 2015). By contrast, poorly controlled diabetes leads to premature death and significant complications such as cardiovascular, neurological and renal diseases (WHO, 2016a; ADA, 2018).

HbA1c is a laboratory test that reflects the average blood glucose levels over the last three months, and it has a strong predictive value for developing diabetes-related complications (ADA, 2014). HbA1c refers to glycated haemoglobin which is a protein in the red blood cells that carries oxygen from the lungs to the body cells. Glycated haemoglobin is formed when haemoglobin binds to glucose. The higher the blood glucose levels, the more glycated haemoglobin is formed (Hillson, 2015). The American Diabetes Association (2017) recommends all patients with $\mathrm{T} 2 \mathrm{DM}$ to perform a HbA1c test twice a year for patients with good glycaemic control levels and four times a year for patients with poorly controlled T2DM. HbA1c levels less than 7\% (good glycaemic control) is recommended for people with diabetes, as it is linked with a reduction in microvascular and long-term macrovascular complications (ADA, 2013). HbA1c results also are reported in millimoles per mol (mmol/mol) which $\mathrm{HbA1c}$ results of $6.5 \%$ and $7 \%$ equal to $48 \mathrm{mmol} / \mathrm{mol}$ and $53 \mathrm{mmol} / \mathrm{mol}$ respectively (Diabetes UK, 2017b).

Poorly controlled T2DM is considered a major public health problem and is associated with adverse outcomes (Ajlouni, K., Khader, Y., Batiehab, A., Ajlouni, H., \& El-Khateeb, M., 2008). As the prevalence rate of poorly controlled T2DM among Jordanian people with T2DM is high, a study was needed to gain a better understanding of the factors associated with poor control of diabetes from the perspectives of people living with T2DM, their family carers and health care professionals. For the purposes of this study, an HbA1c higher than or equal to $7 \%$ was considered as poorly controlled diabetes (ADA, 2013).

In Jordan, although most of the people with T2DM receive their diabetes treatment through primary care services, there is a scarcity of published research about patients and health care professionals' perceptions of T2DM in primary health care (Al-Omari et al., 2009). This study 
was conducted in a primary care setting to add to the limited existing body of knowledge regarding the needs of people with T2DM in Jordan.

I am writing in the first person as I sought to adopt a reflexive approach throughout the conduct of my study. I also refer to people with T2DM who participated in my study as patients as this is how they were referred to by themselves and by the HCWs. Also, I only use the term diabetic patients in the literature review chapter as during my review of research studies about diabetes in Jordan, the majority of them referred to the study participants as diabetic patients, diabetic subjects or study subjects.

The aim and objectives of the study are presented now.

\subsection{Aim and objectives of the study}

Aim

This study aimed to explore the reasons why glycaemic control was poor among a sample of patients with T2DM who used primary care services provided by a selected health care comprehensive centre in a large city in Jordan.

\section{Objectives}

The following objectives were set:

1-To explore the knowledge, attitudes and behaviours of patients with poorly controlled Type2 diabetes and factors affecting adherence to their treatment plans.

2-To identify barriers to Type-2 diabetic care among patients with poor glycaemic control.

3- To explore patients', family carers' and Health care workers' (HCWs) perceptions of the factors that contribute to poor glycaemic levels among patients with T2DM.

4-To identify patients', their family carers' and HCWs' views on factors which might facilitate improved glycaemic control levels among people with T2DM in Jordan.

5-To inform the development of an educational package for patients with T2DM based on information derived from HCWs, patients with poorly controlled T2DM and their family carers to ensure patients' needs and concerns are addressed. 
In order to set the context for the study and to aid understanding of the chapters which follow, I now provide the reader with an overview of the health care system in Jordan.

\subsection{Overview of the health care system in Jordan}

This section provides background information about Jordan and an overview of the health care system, focusing on the primary health care sector in Jordan. Jordan is located in the Middle East region (Figure 1), and in 2017 had a population of 10,053,000 people. Amman is the capital, and it is the largest city in Jordan with a population of 4,226,700 in 2017 (Department of Statistics, 2018). An extended family system constitutes the core of family structures in Jordan. The extended family includes grandparents, aunts, uncles and sometimes other relatives and there is a strong respect and loyalty for older people. In 2015 the average number of family members in Jordan was 4.8 persons (Department of Statistics, 2017). Life expectancy rates for males and females are 72.8 and 74.2 years respectively (Department of Statistics, 2017). Whereas, life expectancy in the UK is 81 years in 2016 (World Bank, 2016). Jordan has witnessed multiple waves of migrations and refugees fleeing in the last 50 years as a result of armed conflicts and insecurity in neighbouring countries. Many Palestinians entered Jordan in 1967 from the West Bank as a result of the Six Days War, and there were 2,175,491 registered Palestinians refugees with the United Nations Relief and Works Agency in Jordan (UNRWA) (UNRWA, 2016).

In 2018, more than 657,628 Syrians and 66,262 Iraqi were registered with the United Nations Refugee Agency in Jordan because of insecurity and war conflict (The UN Refugee Agency, 2018). The Ministry of Health provides free health services to all Syrian refugees who live in the camps (Nazer \& Tuffaha, 2017). All of these crises and events have contributed to an increased demand on the health care system services and facilities in Jordan. Other challenges in the health care system in Jordan are the increasing number of older adults and the increase in non-communicable conditions such as diabetes and hypertension (The Higher Health Council, 2015).

The health care system in Jordan consists of six main sectors: The Ministry of Health (MOH), Royal Medical Services (military), the private sector, public university hospitals, National Centre for Diabetes and the United Nations Relief and Works Agency (UNRWA) (MOH, 2018a). The Ministry of Health is mainly responsible for delivering health care for the majority of people at low costs in Jordan. Home health care is limited in Jordan and most of the patients 
cannot afford home care services and they are not covered by public and private health insurance. Long-term care facilities are not available in Jordan and patients may stay for long periods in the hospitals which increases demand on the health care system services (Nazer \& Tuffaha, 2017).

The majority of primary care centres are managed by The Ministry of Health and are accessible to people living with T2DM in rural and urban areas in Jordan. Primary health care services in Jordan are delivered by 673 health care centres distributed throughout the country. These centres include 110 comprehensive health centres, 377 primary care centres and 186 peripheral health centres $(\mathrm{MOH}, 2018 \mathrm{~b})$. My study was undertaken in one of the comprehensive care centres in a large city. The total number of health care centres in the city under study that provided primary care services was 114 in 2017. They were divided into 23 comprehensive health centres, 63 primary care centres and 28 peripheral care centres ( $\mathrm{MOH}, 2018 \mathrm{~b})$.

The comprehensive health care centres provide general medical services and the main medical specialities such as internal medicine, family medicine, respiratory and infectious diseases along with maternal and child health, dental and public health services (MOH, 2009). The primary care centres only provide general medical services along with maternal and child health, dental and public health services ( $\mathrm{MOH}, 2009)$. The peripheral care centres offer general medical services and public health services (MOH, 2009).

There are multiple types of health insurance available for people in Jordan. The Ministry of Health, Royal Medical Services, university hospitals, UNRWA and private insurance are the main health care bodies that provide health care insurance coverage for people (Nazer \&Tuffaha, 2017). However, some patients have two or more types of insurance which is considered as one of the reasons behind medication wastage and the high national spending on medications mainly for chronic conditions in Jordan (The Higher Health Council, 2015). In addition to having more than one type of health insurance, lack of a shared computerised system adds more burden on the pharmaceutical bill in Jordan. People who work in public and government institutions are provided with insurance for themselves and their family members or 'dependents such as parents, husband, wife and children under age of 18 years as long as these 'dependents' do not have any other insurance' (MOH, 2016). People who are older than 80 years, children under the age of six years, people in less advantaged areas and families with a monthly income of less than 300 Jordanian Dinar (equivalent to £300) who are considered 'poor' have free health insurance (The Higher Health Council, 2015). People who do not have 
The Ministry of Health (MOH) insurance can pay to have annual insurance to receive health care services in hospitals and health care centres managed by the Ministry. For example, people who are older than 60 years have to pay 73 Jordanian Dinar (approximately £75) per year to secure health insurance. $70 \%$ of Jordanians are insured, and the majority of them have insurance with the public sector (Nazer \& Tuffaha, 2017).

The medical exemption is an alternative method for patients who do not have any other type of health insurance. This group of people can apply for the medical exemption provided by the Royal Court. This exemption is mainly offered to people with chronic conditions such as diabetes, heart diseases and hypertension, and the patient is only able to visit the health care centres or hospitals as provided and written in the exemption (Al-Nsour, 2017).

The National Centre for Diabetes, Endocrinology and Genetics (NCDEG) is located in the capital Amman, and it is not accessible to all people, especially those who live in rural areas. NCDEG is considered as the central specialised health care facility for diabetes in Jordan. It is also appointed as an educational institution providing post-graduate studies in multiple fields of diabetes. The vast majority of research studies about diabetes in Jordan are conducted in this centre because of the availability of patients and facilities such as laboratory tests and multiple health care workers. The health care services are integral and provided by a multidisciplinary team of health care providers such as nurses, physicians, clinical nutritionists, educators and podiatrists. Medications and Laboratory tests are available in the centre. Patients who have public insurance can receive care in the centre by paying a percentage fee for the treatment cost (The National Centre for Diabetes, Endocrinology and Genetics, 2018).

The central public health laboratory is the main laboratory in Amman, and it is located in the centre of the city. Patients should visit the central laboratory to do blood tests that are unavailable in their health care centres. The primary documents that a patient has to provide while visiting the health care centre are the insurance card and white card. The patients only have access to one health centre as provided on the white card. At the public health care facilities, all medications are provided free of charge for patients with health insurance (Nazer $\&$ Tuffaha, 2017). The dependants of the insured person have to pay $5 \%$ of the cost of any medication written in the prescription paper (MOH, 2016). 


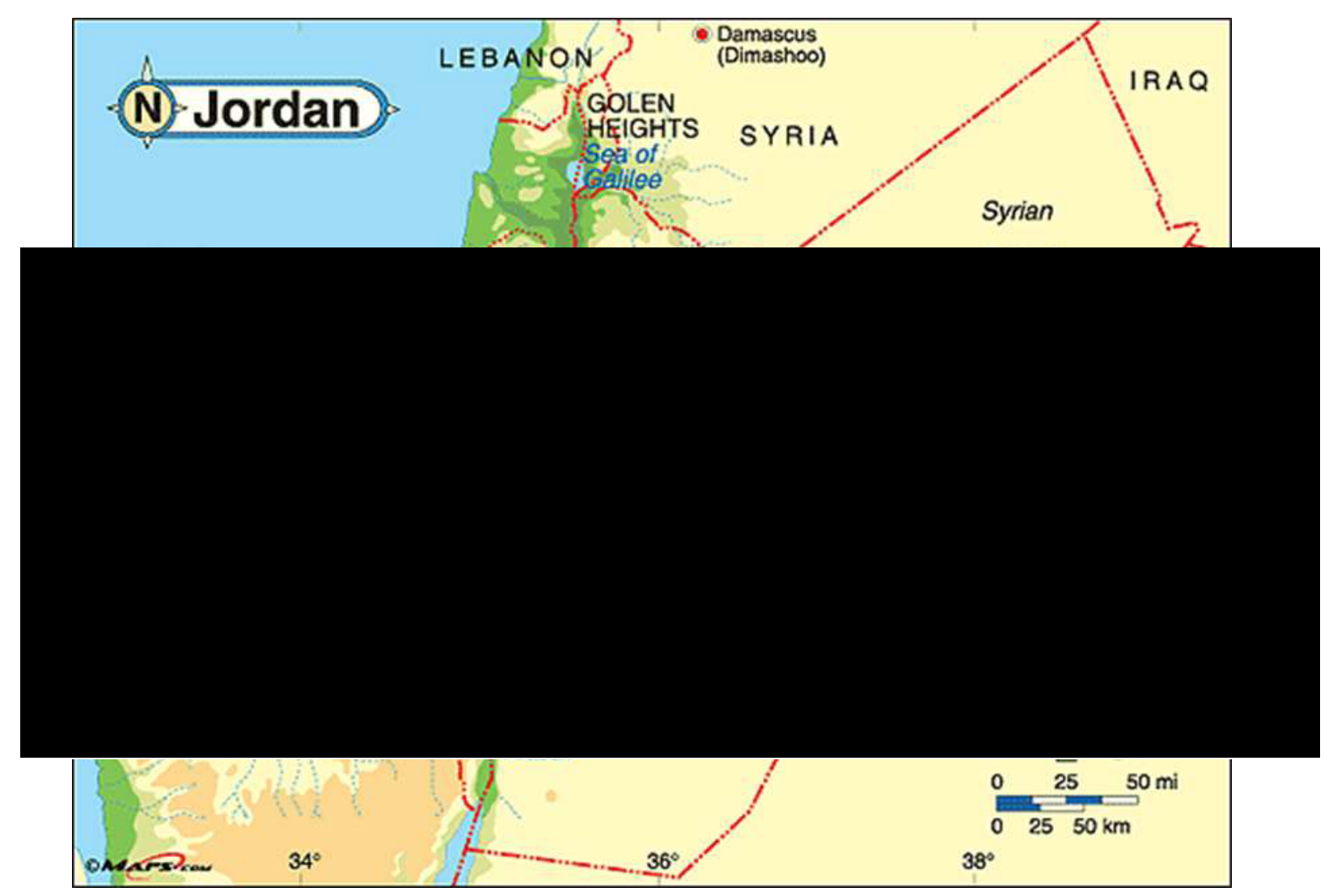

Figure 1 illustrates the map of Jordan (Maps.com, 2011)

This study was conducted in a comprehensive health care centre managed by the Ministry of Health in Jordan. The location of the study setting is presented now.

\subsection{Location of study setting}

A preliminary analysis of the study setting is recommended for researchers before selecting it. This includes: contact with people who are gatekeepers or knowledgeable about the settings, reviewing documents related to the setting and visiting the setting (Hammersley \& Atkinson, 1995). I made initial contact and gained verbal agreement from the director of the selected centre to conduct the research there. The centre director held a positive attitude towards research, and there were positive collegial relationships, and I was introduced to other health care workers, which facilitated field work and enabled me to spend more time in the study setting. This centre was located a short distance away from my home which reduced travel expenses and time and enabled me to visit it multiple times a week.

I have had a clinical background in an Intensive Care unit in one of the largest hospitals in Zarqa, a city in Jordan. I had not worked in the selected centre under study or indeed in any primary care centre in Jordan. The primary care setting was interesting for me, as it is different 
from hospital settings. The system of care, the severity of cases, patients and context are examples of differences between hospitals and primary care centres. My professional experience being a nurse and having worked with patients with T2DM in Jordan stimulated my interest in T2DM. The research topic which is within the area of my professional expertise, and my personal characteristics such as having three family members with Type-2 diabetes, gave me insight into factors that may impact on the management of T2DM.

Availability, feasibility, fewer travel costs and accessibility all may influence a researcher's decision in the selection of research settings (Hammersley \& Atkinson, 1995). This particular centre was appropriate for this study because of the availability of people with poorly controlled T2DM and accessibility to conduct detailed ethnographic fieldwork. The chronic conditions care indicators in the centre, with 55\% $(\mathrm{n}=162)$ of 295 patients with diabetes having their $\mathrm{HbA1c}$ at more than 7\% between January and August 2016 indicated that the centre had a sufficient sample of patients who were poorly controlled. However, incomplete medical records were one of the main concerns observed and mentioned by participants later in the Findings chapter.

The selected centre was a large-sized comprehensive health care centre in a large city, with a considerable number of people accessing its health care services and facilities. 120,578 patients received primary care services from this centre between January and December 2017 of whom 44,651 patients were aged $\geq 45$ years $(\mathrm{MOH}, 2018 \mathrm{~b})$. This centre provided comprehensive health care services for inhabitants who live in three regions of the city.

The centre was established in September 1998 with three floors, and the fourth floor was built in 2004 . The centre provided 24 hours emergency care services, and the family medicine clinics operate from 0800 until 1600. General medical services, family medicine services, maternal and child health services, dental and public health services are examples of services provided by the centre. This centre was also registered as a training centre that provided placements and assessments for nursing students, junior doctors and dentists (Ministry of Health, 2018). The average number of health care workers (HCWs) in the centre on duty per day is provided in table 1:

\begin{tabular}{|l|l|}
\hline Health care workers & Number \\
\hline Family physicians & 5 \\
\hline General practitioners & 11 \\
\hline Lab technicians & 6 \\
\hline
\end{tabular}




\begin{tabular}{|l|l|}
\hline Staff nurses & 5 \\
\hline Auxiliary nurses & 12 \\
\hline Gynaecologist & 1 \\
\hline Midwife & 1 \\
\hline Pharmacists & 6 \\
\hline Dietitians & 2 \\
\hline Receptionists & 5 \\
\hline Diabetic nurse & 1 \\
\hline Orthopaedic doctor & 1 \\
\hline Total & 56 \\
\hline
\end{tabular}

Table 1 illustrates the number of HCWs on duty per day in the studied comprehensive health care centre.

The centre had two access points; the main entrance and the connecting corridor for the use of ambulance staff. There was one lift and staircase to reach all floors. The emergency department and vaccination room were located on the ground floor. Reception, pharmacy, maternal and child department, accounting and laboratory were on the first floor.

The pharmacy and reception departments had two small windows which patients presented at and were in close proximity to each other for female and male patients. There were no seats available on the first floor, so patients had to stand in long queues waiting to receive their medications or their medical records. There was a waiting area with 15 seats on the second floor for patients who were waiting to enter the consultants' rooms. The nursing station was on the second floor next to the family medicine clinics. Each family medicine clinic had an examination bed, two chairs, a desk, and a hand-wash basin. The conference room where I conducted all of the focus groups and interviews was located on the third floor. There were eight family medicine clinics in the centre providing services for people with chronic conditions. During my data collection, there were only 4 or 5 clinics receiving patients due to staff shortages. On average, each clinic provided services for 30 patients with chronic conditions, mainly diabetes and hypertension, every day.

To aid the reader in understanding how the centre functioned the patient's journey through the centre is provided now. 


\subsubsection{The patient's journey through the centre}

During the patient's visit to the centre, the patient had to move between multiple areas within the centre as illustrated in Figure 2. This lengthy process of receiving care added more complexity to the patient's visit to the centre. Furthermore, the patient usually had to stand in a queue in all departments (except in the waiting area on the second floor) which was tiring, especially for older patients.

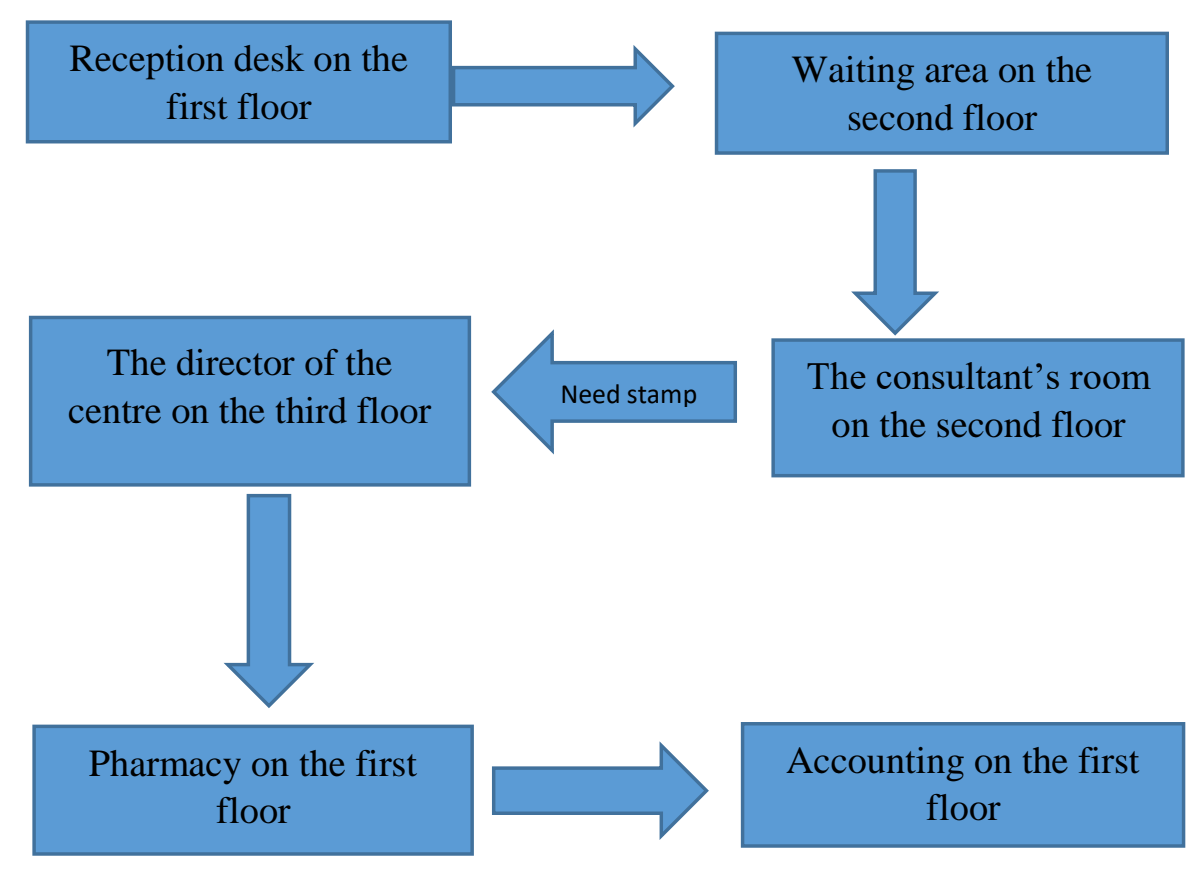

Figure 2 illustrates the process of movement of the patient between different departments within the centre for receiving care.

The patient was usually given a monthly appointment to attend the centre unless he or she was asked to do laboratory tests that needed to be completed and the results shown to the doctor before his or her appointment. The date of the incoming patient's appointment was written by the doctor on his/her appointment card. There was no exact time of the appointment, so patients were seen on a first come first served basis. The patient was able to attend the centre one day before or one day after his/her appointment. For example, if the patient was given an appointment for the 27th of June, he/she could come to the centre on the 26th, 27th or the 28th of June. On the day of appointment the patient had to go through the following process:

First, the patient provided the reception desk (where all patients' medical records were kept) with his/her insurance card and white card (the treatment card that belongs to the selected 
centre). Then the receptionist brought the patient's record and transferred his/her insurance number, type of insurance and other demographic data to the prescription paper (the doctor writes down the patient's medications on it). The number of the clinic where the patient had to go was written on the top of the prescription paper by the receptionist. Then the receptionist gave the patient his/her record and the prescription paper to be handed over to another receptionist (referred to as a 'maid') on the second floor. The patient was asked to stay in the waiting area until the receptionist called his/her name to enter the consultant's room. When the patient entered the consultant's room, he/she had to provide his/her medical record, treatment booklet (it is an official document that encompasses a summary of main demographic data of a patient, diagnosis, time of the visit and medications), prescription paper and appointment card. When the doctor wrote a request for laboratory tests to be done mainly in the central laboratory, the patient had to obtain a stamp from the director of the centre. Additionally, if the request included a vitamin $\mathrm{D}$ test, the patient had to receive a signature from the orthopaedic consultant who worked only on Monday and Thursday. The majority of laboratory tests such as $\mathrm{HbA1c}$, kidney function test, liver function test and vitamin levels were done in the central laboratory.

After visiting the doctor, the patient went to the pharmacy for costing the medications or prescriptions then he/she attended the accounting department before going back to the pharmacy to collect his or her medications. The average length of a patient's visit to any health care centre in Jordan according to the Ministry of Health official website (2018) is 20 minutes without requesting laboratory tests. However, during my data collection, I found that the patients experienced different types of barriers (Figure 3) and they usually spend 2 to 4 hours in the centre which was more than what is expected according to the Ministry's website.

There was a short cut for patients with disabilities, older patients and patients with chronic renal failure. With doctors' permission, these patients were given their records rather they visited the reception desk to facilitate their access to be seen by the doctors. This group of patients should not visit the reception desk as they went directly to the waiting area on the second floor until they were called by the receptionist to enter the consultant's room. After visiting the doctor, these patients were given priority over other patients for receiving their medications from the pharmacy to reduce the length of their visit to the centre. 


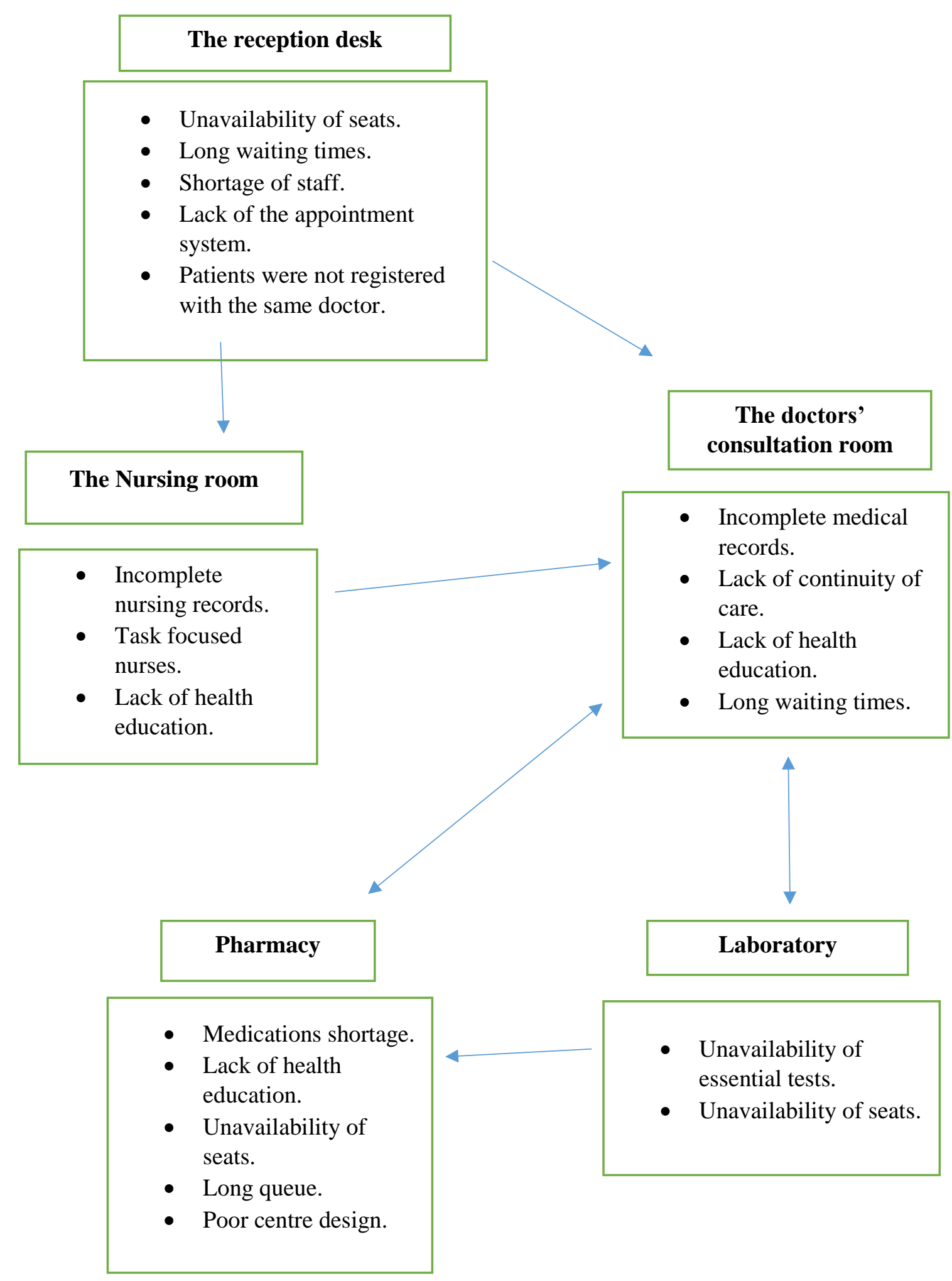

Figure 3 illustrates the barriers that patients may experience during their visit to the centre.

Having orientated the reader to the research setting and the process of the patient's visit to the centre, I now orientate the reader to research design and layout of the thesis. 


\subsection{The layout of the thesis}

This study is divided into nine chapters. Chapter Two provides a critical review of the literature.

Chapter Three describes the methodological approach used in this study and the tools used to collect data. It describes the rationale for choosing ethnography, the aims and objectives, research paradigm, study design, study setting, sampling method, ethical considerations, recruitment process, methods of data collection, study participants, data analysis and methodological rigour. Chapter Three also provides a description of the theoretical perspectives that were considered to offer appropriate analytical insights into my data. It presents a number of theories that have been drawn upon to aid understanding of the factors associated with poor glycaemic control amongst patients with T2DM.

Chapter Four presents the characteristics of the participants in this study and provides an overview of the four main themes which constitute the four findings and discussion chapters.

Chapter Five titled 'Attitudes, knowledge and perceptions of patients regarding Type-2 diabetes' presents and discusses the subthemes and categories related to the patients' attitudes, knowledge and perceptions regarding diabetes. It also provides data related to patients' perceptions towards insulin and self-monitoring blood glucose levels (SMBG), and misconceptions about diabetes and its management.

In Chapter Six, the social consequences of diabetes on patients and the influence of sociocultural factors on patients' diabetes management behaviours (including diet, medications and physical activity) are presented and discussed. Food traditions and customs in Jordan and social factors influencing physical activity and adherence to the medication regimen are presented. Data related to herbal usage, types of plants and herbs and the reasons for utilising them are also presented and discussed.

In Chapter Seven, the challenges that hindered the management of diabetes at two main levels of care; the health care system and health care workers (HCWs) in the comprehensive health care centre are presented. Chapter Eight discusses the subthemes and categories related to the facilitators as suggested by patients, family carers and health care workers to improve blood glucose levels among patients. 
Finally, in Chapter Nine, a summary of the main findings is presented, and the limitations of the study are discussed. This is followed by a discussion of the relevance and implications of study findings and recommendations for future research, practice, education and policy.

In summary, I have provided the reader with an introduction to the thesis, an overview of the health care system, research setting and process of the patient's visit to the centre. The next chapter reviews the literature on Type- 2 diabetes and its management. 


\section{CHAPTER TWO}

\section{LITERATURE REVIEW}

\subsection{Introduction}

This chapter includes information about the literature that was reviewed and accessed critically during the conduct of this study. This chapter begins with describing the literature search strategy, the critiquing tool used, the aim of the literature review, inclusion and exclusion criteria and shortfalls identified in the literature. This review is organised by thematic separation into four major themes. The first theme discusses the extent of T2DM and significant diabetes related-complications in Jordan. The second theme addresses diabetes selfmanagement behaviours (diet, physical activity, adherence to medications, monitoring blood glucose and foot care). The third theme focuses on the knowledge, perceptions and attitudes of patients towards their condition, scarcity of qualitative studies and factors associated with negative and positive attitudes. The fourth theme concentrates on the literature about complementary medicine and the reasons for herbal use among patients in the previous studies. In order to conduct the literature review, I used the following literature search strategy which is discussed now.

\subsubsection{Literature search strategy}

The literature review began in October 2015 when I enrolled in my PhD studies, and has been ongoing with regular updating and reviewing of the literature to ensure up-to-date information has been included. The review used a narrative approach and synthesised the evidence from research and literature reviews published between 1998 and 2018.

The references retrieved from the literature searches were carefully evaluated and analysed to identify all references meeting the inclusion criteria for the literature review. Critical Appraisal Skills Programme (CASP) tools were used to analyse, critique and evaluate the published quantitative and qualitative literature. These tools consist of questions and sub-questions explicitly designed to assess the quality of published research according to the research design (Critical Appraisal Skills Programme, 2018).

This literature review aimed to identify the literature regarding factors associated with poorly controlled T2DM. The search strategy used keywords related to the aims and objectives of this 
study. During this research study, more themes and keywords have emerged which resulted in revising, expanding and adding more keywords to the search strategy. Appendix 19 illustrates all the keywords and the search terms used in the search process.

The key electronic databases used for literature searching included MEDLINE, Google, Google Scholar, Swansea University IFind research, Web of Science, PsycInfo, PubMed, International Bibliography of the Social Sciences (IBSS) and Applied Social Sciences Index and Abstracts (ASSIA). Research articles, abstracts, literature reviews, the reference list of included studies, reports and documents related to the research subject were reviewed carefully in these databases. Also, the official publications of organisations such as the World Health Organisation (www.who.int), Diabetes UK (www.diabetes.org.uk), American Diabetes Association (www.diabetes.org) and International Diabetes Association (www.idf.org) were also accessed. Also, my supervisors, as experts in the primary care and chronic conditions helped me in identifying relevant articles.

The search relating to the aims and objectives of this study was conducted between October 2015 and October 2018. The primary literature search language was in English. However, literature in the Arabic language was also reviewed. I reviewed and translated Arabic published reports about the health care system, primary care services, health promotion and cultural norms that were found on the Jordanian government websites such as the Ministry of Health (www.moh.gov.jo) and Department of Statistics (www.dos.gov.jo). National Centre for Diabetes, Endocrinology and Genetics in Jordan (www.ncd.org.jo), fact sheets, policy statements and Jordanian newspapers were also accessed for published records about diabetes in Jordan.

The literature search yielded 667 studies in total. Titles and abstracts were examined to identify eligibility for full screening. Studies were screened by title as the first step, then the abstract. A total of 620 studies were excluded from the review based on the subsequent sifting process and inclusion criteria set out previously. Exclusion reasons varied, for example, having irrelevant aims, including participants who are not diagnosed with diabetes or chronic conditions and including patients with Type-1 diabetes only were excluded. Studies were also excluded due to unavailability of full access to the study, the quality of the study, the inclusion of irrelevant participants such as pregnant women and women with gestational diabetes.

A total of 47 studies were eligible for the literature analysis (Figure 4) focusing on barriers to glycaemic control and adherence to diabetes management in T2DM in Jordan. A total of 31 
studies were identified from Jordan and 16 studies were included from: Kuwait (one study), Malaysia (one), Saudi Arabia (four), Turkey (one), Israel (one), Iraq (one), and the United Arab Emirates (five). One study was conducted across the European countries, and one study was identified from the United States of America. Also, a full description of the studies included in the literature review is provided in Appendix 21.

To broaden my literature search, I adopted multiple search strategies. Firstly, all studies related to the aim and objectives of this study among adults with diabetes in Jordan and Middle Eastern countries were eligible for inclusion. The inclusion criteria required that the study population be adults (18 years and older) with diabetes. Secondly, I divided the research topic into parts, concepts and terms. Thirdly, I used different forms of keywords for each concept such as synonyms, different terminology, alternative words and alternative spellings. Fourthly, I reviewed literature from multiple regions and countries throughout the world. When there was limited identified literature about a particular topic area such as patients' perceptions and attitudes towards their condition in Jordan, I modified and broadened my search to include relevant publications in the neighbouring countries that share similar cultures such as Saudi Arabia, Oman, Iraq, Iran, Kuwait and the United Arab Emirates (UAE).

The literature search and review identified that a cross-sectional survey is the most popular research design in the published articles and papers related to diabetes in Jordan (e.g. Khatib et al., 2006; Al-Bdour, Al-Till, \& Abu Samra, 2008; Rabiu et al., 2015; El-Qudah, 2016; Bakkar, Haddad, \& Gammoh, 2017). Also, most of these studies focused on assessing the prevalence of diabetes and behaviours such as adherence to diabetes management behaviours, T2DM and diabetic foot complications (e.g. Ajlouni et al., 2008; Albikawi \& Abuadas, 2015; Obeidat, Ahmad, Haddad, \& Azzeh, 2015; Al-Shudifat et al., 2017; Khawaja et al., 2018). There is a shortfall in qualitative research in exploring the factors associated with the management of T2DM among patients, and few qualitative studies are also available in the literature in Jordan.

Few studies were identified that had researched diabetes in primary and comprehensive care settings in Jordan. Furthermore, no published qualitative studies that explored attitudes and perceptions of patients with T2DM were found in the Jordanian literature. To address this, I expanded my search to include literature from other Middle East countries with similar cultural and social backgrounds, for example, Saudi Arabia, Oman, Iran, Kuwait and the UAE. 


\section{Summary of the literature search procedure}

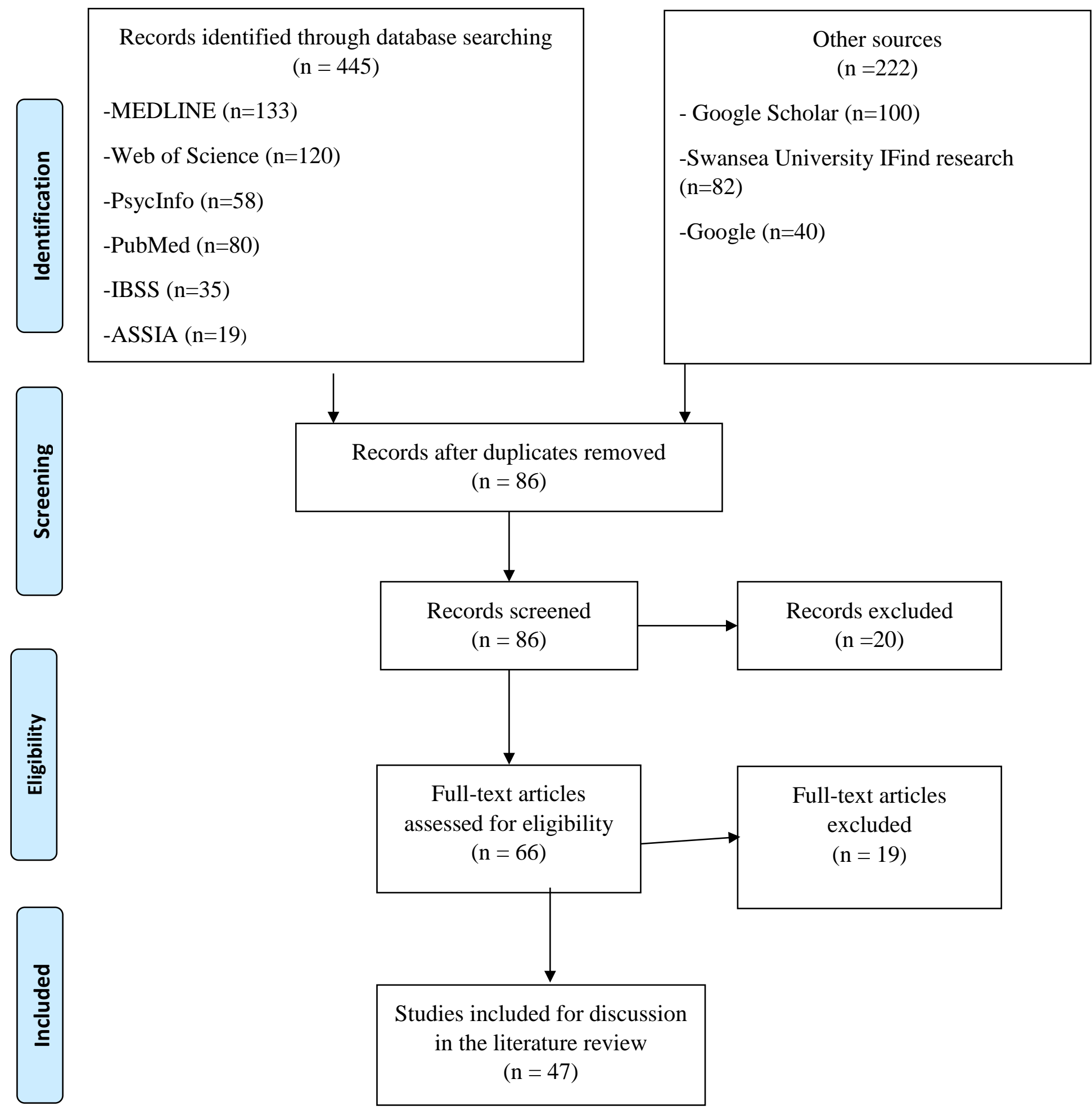

Figure 4 PRISMA flow diagram showing the selection process of the included studies in the literature review 
The next section I review the literature that has investigated the extent of T2DM and its complications in Jordan.

\subsection{The extent of Type-2 diabetes and its complications in Jordan}

Chronic conditions are considered to be the leading cause of mortality in Jordan (Nazer \& Tuffaha, 2017). Jordan has witnessed demographic changes such as increasing life expectancy rates and an epidemiological transition as the burden of communicable diseases has reduced compared with the high prevalence rates of non-communicable conditions namely cardiovascular diseases and diabetes (Al-Nsour et al., 2012). These changes in the Jordanian health care profile have an impact on the public health policies and priorities in Jordan to shift the focus more towards the non-communicable conditions. For instance, prevention of diabetes mellitus is addressed as one of the research priorities in Jordan (The Higher Council for Science and Technology, 2010). Diabetes mellitus was the third leading cause of mortality after ischaemic heart disease and stroke in Jordan in 2015 (WHO, 2015).

A review of the epidemiological literature in Jordan and international health organisations showed that the high prevalence rate of diabetes among Jordanians is higher than the prevalence rate of diabetes globally (8.5\%) (WHO, 2016a). Estimations of the prevalence of diabetes in Jordan were reported variably to range from 9-17.1\% (Ajlouni et al., 2008). For instance, the prevalence rate of diabetes in Jordan increased from 13\% in 1998 (Ajlouni, Jaddou, \& Batieha, 1998) to $17.1 \%$ in 2008 (Ajlouni et al., 2008). The World Health Organisation (2016) also reported that the prevalence rates of diabetes and diabetes-associated risk factors such as being overweight and obesity in Jordan were 13.1\%, 62.3\% and 28.1\% respectively. For example, the prevalence rate of diabetes was more than $7 \%$ and more than half of adults were overweight or obese and $48 \%$ of adults were being treated for a chronic illness in Wales (Wallace \& Kenkre, 2015; Diabetes Wales, 2018). Based on a report provided by International Diabetes Federation in 2017, it is estimated that the prevalence rates of diabetes in Jordan and its neighbouring Arab countries such as Saudi Arabia, Qatar and the UAE were $9.5 \%, 18.5 \%, 14.1 \%$ and $15.6 \%$ respectively (IDF, 2017).

During the last 30 years, people in Jordan have experienced behavioural and lifestyle changes (Boutayeb et al., 2012). These changes have led to a rise in the prevalence of all types of diabetes in Jordan and also an increase in the risk factors associated with diabetes such as poor quality nutrition, more consumption of fast food, low intake of fruits and vegetables, reduced physical activity, obesity, smoking and poor awareness of a healthy weight among people in 
Jordan (Zindah, Belbeisi, Walke, \& Mokdad, 2008; Boutayeb et al., 2012; Al-Shudifat et al., 2017). Additionally, inviting guests for sharing meals, which include high-calorie content, is considered as a highly valued socio-cultural habit in the Arab world and may contribute to the high prevalence of diabetes in the Arab region (Boutayeb et al., 2012). Lack of knowledge about healthy behaviours and the role of social activities also contributed to a sedentary lifestyle in Arab countries (Sharara, Akik, Ghattas, \& Obermeyer, 2018). Previous studies (AlBdour et al., 2008, Ali, Hajeri, Khader, Shegem, \& Ajlouni, 2008; Yasein \& Masa'd, 2011; Obeidat et al., 2015; Khawaja et al., 2018) reported that the high prevalence rate of diabetes is associated with many negative consequences among patients with diabetes in Jordan. These complications are contributors to the burden of the condition in Jordan. For instance, metabolic syndrome, sexual dysfunction, periodontal disease, diabetic neuropathy and diabetic retinopathy are examples of complications among patients with diabetes in Jordan.

Metabolic syndrome (MS) is a group of cardiovascular risk factors including T2DM, prediabetes, high blood pressure, abdominal obesity and high cholesterol (IDF, 2013). The prevalence rate of metabolic syndrome in Jordan increased from 36.3\% in 2008 (Khader, Khatatbeh, El-Salem, Amarin, \& Bateiha, 2008) to $37.4 \%$ in 2010 and 51\% in 2015 (Yasein \& Masa'd, 2011; Obeidat et al., 2015). These studies will be evaluated and discussed later in this section. These studies used different sets of criteria as cut-off points for metabolic abnormalities instead of using one criterion. The methodologies of these studies are reported in more details later in this chapter.

A cross-sectional survey of 730 patients recruited by a random sampling from all patients aged $\geq 25$ years attending the family practice clinic at Jordan University Hospital showed that prevalence rate of metabolic syndrome was $37.4 \%$ in 2010 and that T2DM was one of the contributing factors to metabolic syndrome among 157 patients in Jordan (Yasein \& Masa'd, 2011). Yasein and Masa'd (2011) revealed in their study findings that T2DM was one of the most common chronic conditions among patients with metabolic syndrome. However, the sampling strategy was random by recruiting patients from one clinic and not from other health care settings which limits the generalisability of the findings.

These findings are similar to another cross-sectional study with a sample size of 280 patients with T2DM which showed that $83.2 \%$ of participants had metabolic syndrome according to the International Diabetes Federation criteria (Alhabashneh, Khader, Herra, \& Asa'Ad, 2015). The findings from previous studies (Khader et al., 2008; Yasein \& Masa'd, 2011; Alhabashneh et 
al., 2015; Obeidat et al., 2015) regarding metabolic syndrome in Jordan added valuable information to the existing literature. However, using different cut-off points of defining metabolic syndrome impedes comparing the findings of these studies and makes it difficult to generalise.

Conducting studies regarding the sexual life of patients with diabetes may face challenges. For instance, patients may feel embarrassed when they talk about their sexuality or sexual difficulties in front of health care providers (Khatib et al., 2006). Erectile dysfunction is one of the common complications of diabetes among men, and it is a sign of cardiovascular diseases and premature mortality (Hillson, 2015). However, there is a scarcity of published studies regarding sexual function among patients with diabetes in Jordan. I only found two published papers (Khatib et al., 2006 \& Ali et al., 2008). Both of them were prevalence studies focusing on physiological factors.

Khatib et al. (2006) conducted a survey to identify the prevalence rate of erectile dysfunction among 988 patients with all types of diabetes. The mean age of participants was 57 years. The structured interviews were used and based on the International Index of Erectile Function (Rosen, Brown, Heiman, \& Leib, 2000). This questionnaire assesses erectile function, intercourse satisfaction, orgasmic function, sexual desire and overall satisfaction. A local committee evaluated the appropriateness and clarity of the questionnaire for the Jordanian culture. $62 \%$ were found to have erectile dysfunction and $75 \%(n=741)$ had a HbA1c $>7 \%$. The high prevalence of erectile dysfunction (62\%) was due to the high number of poorly controlled cases and the old ages of participants. Female sexual dysfunction among people with diabetes has also been studied in Jordan.

Female Sexual dysfunction was higher in diabetic female patients with diabetes than females without diabetes (Ali et al., 2008). The Female Sexual Function Index questionnaire (Rosen et al., 2000) was used to collect data about desire, arousal, lubrication, orgasm, satisfaction and pain from 613 female patients with all types of diabetes and 524 women without diabetes. The criteria for defining sexual dysfunction was not explained, and the tool used was not tested or piloted by female Jordanians. The findings of this study were superficial, providing numbers without proper rationale, and this study also did not consider the factors that may lead to sexual dysfunction. Furthermore, administration of the Female Sexual Function Index questionnaire raises concerns regarding the appropriateness of this American questionnaire for the Jordanian context especially in culturally sensitive topics such as sex. These studies were included in this 
review to show that sexual dysfunction complications are prevalent among patients with diabetes in Jordan (Khatib et al., 2006; Ali et al., 2008).

Diabetic retinopathy (an eye disease) is a common microvascular complication among patients with diabetes due to unstable blood glucose levels and longer duration of diabetes (Hillson, 2015). Diabetic retinopathy (DR) formed $2.6 \%$ of causes of blindness worldwide in 2010 (WHO, 2016a). Prevalence of diabetic retinopathy in Jordan is high, 34.1\% in 2008 and 48.4\% in 2015 (Al-Bdour et al., 2008, Al-Amer et al., 2008; Rabiu, Al Bdour, Abu Ameerh, \& Jadoon, 2015). These three studies identified more than half of their participants had a $\mathrm{HbA1c} \geq 7 \%$ which is considered as a risk factor for the development of diabetic retinopathy (IDF, 2017). Al-Bdour et al., (2008) conducted a cross-sectional study of 1961 diabetic patients living in Jordan. $64.1 \%(n=1257)$ were found to have one type of diabetic retinopathy, and there was a significant association between diabetic retinopathy with poorly controlled diabetes and longer duration of disease (Al-Bdour et al., 2008). Full eye examination, biochemical measurements and medical assessment were used to identify the main risk factors for diabetic retinopathy. The sampling method was not explained in this study which may impede transferability of the findings to other patients with diabetes in Jordan.

Al-Amer et al. (2008) reported that $34.1 \%$ of their random sample of 1000 patients with T2DM had diabetic retinopathy. The study was a cross-sectional survey and data were collected by eye examination and biochemical measurements. The mean $\mathrm{HbA1c}$ was $8.3 \%$. $77.5 \%$ were found to have a $\mathrm{HbAlc} \geq 7 \%$, the mean age was 57.8 years, and the mean duration of disease was 9.6 years. The high number of poorly controlled cases, the old ages of participants and the long duration of T2DM might explain the high prevalence of diabetic retinopathy amongst the sample in this study.

Another cross-sectional study aimed to identify the prevalence of blindness, visual impairment, diabetes and diabetic retinopathy in north Jordan (Rabiu et al., 2015). A total of 3638 out of the 3780 eligible participants were examined. Untreated cataract and diabetic retinopathy were the major causes of blindness, accounting for $46.7 \%$ and $33.2 \%$ of total blindness cases, respectively. The prevalence of diabetes mellitus was $28.6 \%$ among the study participants. The prevalence of diabetic retinopathy among patients with diabetes was 48.4\% (Rabiu et al., 2015).

By contrast, another cross-sectional survey reported a lower prevalence of diabetic retinopathy of $7.9 \%$ amongst a randomly selected sample of 127 people with T2DM recruited from the National Diabetes Centre and diabetes clinic in Jordan (Jammal et al., 2013). After comparison 
with the other published studies in Jordan, this lower prevalence rate may be explained by the differences within the samples with the participants in the latter study having only had T2DM for less than six months, and the mean age was $49.7 \pm 10$ years. Longer duration of diabetes and an older age are the main risk factors for the development of diabetic retinopathy (Hillson, 2015).

Obesity and being overweight are the main risk factors for developing diabetes and its complications (WHO, 2016a). 58\% of the global burden of T2DM has been attributed to obesity (Helman, 2007). Obesity is defined as an excessive accumulation of fat in the body. In addition to the percentage of body fat, the distribution of fat in the body (android or upper body obesity) is considered as a major contributor to the development of diabetes-related complications (Unger, 2012). Body mass index (BMI) is a simple formula used to categorise overweight and obesity. It is calculated as a person's weight in kilograms divided by the square of his/her height in meters $(\mathrm{kg} / \mathrm{m} 2)$. A person is considered obese if his/her BMI $\geq 30$ (Nuttall, 2015). However, BMI is only an indicator of excess weight. BMI does not also provide information about muscle mass, bone mass, the distribution and amount of fat in the body (NHS, 2015). Furthermore, age, gender and muscle mass are not taken into account in BMI measurement (NHS, 2015). For instance, people with high muscle mass may have a high BMI and classified as obese or overweight (Nuttall, 2015). Also, there are BMI reference scales for different ethnic groups are likely to significantly affect the interpretation of BMI data (Nuttall, 2015).

According to the World Health Organisation statistics, in 2016, $21 \%$ of male Jordanians and $35.6 \%$ of female Jordanians were considered obese (WHO, 2016b). The prevalence rate of overweight is also high at $58.9 \%$ for males and $66 \%$ for females in Jordan in 2016 (WHO, 2016b). Several factors contribute to an increase in the prevalence of obesity and overweight among people in Jordan (Al Nsour et al., 2012). For instance, lack of knowledge about healthy behaviours and the role of social activities that are associated with consuming a high caloriedense food, sedentary lifestyle and physical inactivity (Al Nsour et al., 2012; Sharara et al., 2018). As populations in Jordan become more urbanised, and as lifestyles shift towards increased physical inactivity and increased food consumption, the prevalence of obesity and overweight is expected to rise (El-Qudah, 2016). There is also a cultural belief regarding a positive image of plumpness among most people in Arab countries (Helman, 2007; Madanat, Troutman, \& Al-Madi, 2008). This form of obesity is called 'cultural obesity', and for women, it means that a woman with a large body size is viewed as being a symbol of beauty and an 
indication of fertility and healthiness (Helman, 2007). For men, it indicates a high socioeconomic status and good health (Madanat et al., 2008).

The high prevalence of obesity among patients with diabetes suggests there is a significant association between diabetes and obesity in Jordan (Al Nsour et al., 2012 \& El-Qudah, 2016).). Patients with T2DM rely on using cars and other methods of transportation instead of walking even for short distances (Irshaid, 2014). These features of a sedentary lifestyle besides physical inactivity and poor diet may contribute to high levels of obesity among patients with T2DM. $67.2 \%$ of 195 diabetic subjects were classified as obese (Ajlouni et al., 2008). This result is similar to the findings from another cross-sectional study which aimed to identify the factors associated with poorly controlled T2DM in Jordan which reported that $69.5 \%$ of 532 obese patients with T2DM were poorly controlled cases (Khattab, Khader, Al-Khawaldeh, \& Ajlouni, 2010). Another cross-sectional study explored dietary knowledge among a convenience sample of 180 females with diabetes recruited by convenience sample from different hospitals in Jordan (El-Qudah, 2016). Most of the patients had diabetes for more than two years $(78.3 \%)$ and had a positive family history of diabetes as a first-degree relative. Approximately a half of them suffered from diabetes-related health diseases and about half of the patients were overweight, and about 32\% were obese (El-Qudah, 2016). Only women with diabetes were included in this study which limited generalisability of findings to other patients.

Over recent years Jordan has witnessed not only the high prevalence of T2DM but also an increase in the number of poorly controlled T2DM cases.

Several studies have been conducted in Jordan which showed the prevalence of poorly controlled diabetes, of all types among patients with diabetes, was high (MOH \& Arbaji, 2005; Khatib et al., 2006; Al-Amer, Sobeh, Zayed,\& Al-domi, 2011; Alhabashneh et al., 2015). Other authors (Ajlouni et al., 2008; Al Omari et al., 2009; Khattab et al., 2010; Al-Khawaldeh, AlHassan, \& Froelicher, 2012; Bawadi, Abu-Jamous, \& Tayyem, 2014; Khawaja et al., 2018) aimed to conduct their studies including only patients with T2DM. These studies reported that the prevalence rate of poorly controlled T2DM was also high ranging from 43.9 to $78.2 \%$. These studies are reported and criticised throughout this chapter.

Of the Jordanian studies that reported the prevalence of poorly controlled diabetes, only a few were conducted in primary care settings such as diabetic clinics at the primary care level, comprehensive care centres and primary care centres (MOH \& Arbaji, 2005; Ajlouni et al., 2008; Al Omari et al., 2009). Furthermore, the criteria for defining poorly controlled diabetes 
was variable among these studies with two studies using an $\mathrm{HbA} 1 \mathrm{c}>7.5 \%$ as the cut-off point for determining poorly controlled diabetes (Ajlouni et al., 2008; Al-Khawaldeh et al., 2012).

In comparison, a primary care survey (2005) showed that $63.4 \%$ of 1150 patients with diabetes were poorly controlled cases; poorly controlled diabetes in this survey was defined as an $\mathrm{HbA} 1 \mathrm{c} \geq 7 \%$ (MOH and Arbaji, 2005). Five further studies also used a HbAlc $\geq 7 \%$ as a cutoff point for poor glycaemic levels (Khatib et al., 2006; Al Omari et al., 2009; Khattab et al., 2010; Al-Amer et al., 2011 \& Khawaja et al., 2018). Only one study used a HbA1c > 8\% as a cut-off point to define poorly controlled diabetes and found that $50 \%$ of 750 patients with T2DM had poor glycaemic control levels (Bawadi et al., 2014). Alhabashneh et al. (2015, p. 3) used the term "well controlled" T2DM to refer to a HbAlc $\leq 6.5 \%$. In her study findings, she reported only $21.8 \%$ of 280 patients with T2DM were well controlled. Using different cutoff points for HbA1c impedes comparing the findings of these studies.

Ajlouni et al. (2008) conducted a cross-sectional survey to discover the prevalence rate of T2DM among Jordanians over ten years in a comprehensive health centre in Sarih a town in the north of Jordan, with a randomly selected sample of 1121 participants who were all aged $\geq 25$ years. Data were collected by sociodemographic questionnaire, anthropometric (weight, height, hip and waist circumferences) and biochemical (HbA1c, fasting blood glucose and lipids profile) measurements. The study findings identified 195 participants who had T2DM, of whom 79 (54\%) were poorly controlled cases. Unsatisfactory glycaemic control was defined in the study as a $\mathrm{HbA} 1 \mathrm{c}>7.5 \%$.

A longer duration of T2DM and type of treatment have been found to be associated with poor glycaemic control levels in Jordan (Al-Omari et al., 2009). Khattab et al. (2010) claimed poor adherence to self-care management behaviours, negative attitudes toward diabetes and longer duration of T2DM were associated with poor glycaemic control levels among people with T2DM. This study was conducted in the National Diabetes Centre in Amman, Jordan with a systematic random sample of 917 patients with T2DM. Sociodemographic data, clinical characteristics and diabetes self-management behaviours were collected. Barriers to diabetes care adherence scale, attitudes towards diabetes scale, family and friends support about diabetes scale and medication adherence index were also used in the study. Additionally, all blood glucose levels and lipids profiles were taken from patients' files. $65.1 \%$ of participants were identified as having poorly controlled T2DM with a $\mathrm{HbA} 1 \mathrm{c} \geq 7 \%$. 
T2DM is a chronic condition which requires daily care and following multiple self management behaviours. Diabetes self-management behaviours include diet, physical activity, adherence to medications, monitoring blood glucose and foot care (Albikawi \& Abuadas, 2015). These behaviours will be discussed next.

\subsection{Diabetes self-management behaviours}

\subsubsection{Diet}

Poor diet is one of the modifiable risk factors in addressing the increase of prevalence of T2DM among people living in Jordan. T2DM dietary management aims to improve insulin sensitivity, lose weight and maintain good glycaemic and cholesterol levels (Unger, 2012; Hillson, 2015). Diet is not only what patients with diabetes eat, but also the portion of food that they consume in each meal (Hillson, 2015). There have been many studies which have explored the diet among patients with diabetes in Jordan (Al Omari et al., 2009; Khattab et al., 2010; Al-Amer et al., 2011; Al Jamal \& Ibrahim, 2011; Al-Sarihin, Bani-Khaled, Haddad, \& Althwabia, 2012; Bawadi et al., 2014; El-Qudah, 2016).

A review of the literature in Jordan (Khattab et al., 2010; Al-Amer et al., 2011) showed that most of the patients with diabetes did not follow a healthy, balanced diet as recommended by the dietitian and being reluctant to follow the diet was associated with poorly controlled diabetes. Cultural, religious, social and personal factors may influence patients' adherence to a healthy diet in Jordan and Arab countries (Sharara et al., 2018). These factors will be discussed later in this section. Furthermore, since there is a lack of national dietary guidelines for patients with diabetes in Jordan (Bawadi et al., 2014), patients with T2DM may be unaware of what constitutes a healthy diet.

Khattab et al. (2010) conducted a cross-sectional study to identify the factors associated with poorly controlled T2DM in Jordan. The study findings reported that poor adherence to the recommended diabetic diet was associated with poor glycaemic control levels among patients with T2DM and $81.4 \%$ of the 917 participants did not follow the diabetic diet. This result is similar to another cross-sectional study with a sample size of 649 patients with diabetes in Jordan which showed $64.7 \%$ of participants did not follow the diabetic diet and $70.9 \%$ of the participants were poorly controlled (Al-Amer et al., 2011). Following the recommended diabetic diet in both studies referred to people with diabetes who followed the diabetic diet as 
recommended by a dietitian $\geq$ three days in the last seven days. The reason for using this criterion was not justified in these studies. Furthermore, the data regarding diabetic diet was collected by yes or no answer (superficial data) and based on a self-report which might lead to recall bias. The exclusion criteria in Khattab et al. (2010)'s study was also not fully described which means it might have included people with cognitive impairment having difficulty in remembering their diet plans in the previous seven days.

Adherence to dietary management plan is an essential part of a patients' daily routine and can be the only treatment needed for some patients with T2DM. Al Omari et al. (2009) in his crosssectional study reported that $13 \%$ of 337 Type- 2 diabetic patients managed their diabetes at a good level (the mean HbA1c was $6.4 \%$ through having a diabetic diet alone. The data were collected by reviewing the medical records of all Type- 2 diabetic patients who attended a diabetic centre at the primary care level in the university teaching family health centre between September 2007 and December 2008.

Al-Sarihin et al. (2012) also reported similar findings in his cross-sectional survey. These study findings revealed $12 \%$ of a convenience sample of 100 diabetic patients (Type $1 \& 2$ ) controlled their diabetes by having a diabetic diet only. This study did not provide information regarding the percentage of people with T2DM in the selected sample. Therefore, this result is not clear because it should be $12 \%$ of people with T2DM since people with Type -1 diabetes are not able to manage their diabetes through diet alone. Overall the sample size is small which limits the generalisability of the findings.

The human body needs fats that should principally come from monounsaturated and polyunsaturated sources, for instance, fish oils, avocado oil and olive oil (Hillson, 2015). Olive oil is a key component of the Jordanian daily diet due to cultural and religious beliefs (Bawadi et al., 2014). Olive oil is a good source of monounsaturated fats, which are considered 'good' fats, which can reduce the levels of cholesterol (ADA, 2015b) and can improve insulin sensitivity among people with diabetes (Unger, 2012). Bawadi et al. (2014) found a high intake of monounsaturated fats was attributed to olive oil consumption among patients with diabetes in Jordan. Al Jamal and Ibrahim (2011) conducted a pre/post randomised trial over four weeks to study the impact of olive oil on blood glucose and lipids levels among a randomly selected sample of 45 subjects with T2DM. Study participants were asked to consume $30 \mathrm{ml}$ of olive oil distributed after the three meals, breakfast, lunch and dinner. Data were collected by biochemical (fasting blood glucose and lipids profile) measurements at the starting day and the 
end of week 4. The study findings identified a significant improvement in fasting blood glucose and lipids levels due to the consumption of olive oil. However, this study did not contain information regarding key features of the sample such as the duration of diabetes, HbA1c levels and type of treatment which might have an impact on the study findings. The author also used high levels of fasting blood glucose, $160-320 \mathrm{mg} / \mathrm{dl}$, as a marker for poorly controlled T2DM to recruit patients with T2DM. Fasting blood glucose does not reflect the long-term management of diabetes since it varies from day to day and reflects the level of blood glucose in one day only (Hillson, 2015). HbA1c readings would be more accurate which would mean that this study needs to be conducted over 3 to 4 months to reveal more accurate and stable results. Furthermore, multiple confounding variables were not controlled in this study such as diet, exercise, type of treatment and adherence to medications which might influence the impact of olive oil on blood glucose and lipids levels. This study could be improved if these variables were controlled and the collected data were expanded to include data about these factors.

People in Jordan tend to consume a lot of a famous Jordanian cuisine called mansaf, and it is considered to be integral to certain occasions and celebrations such as weddings, funerals and a graduation ceremony (MOH, 2012). Lunch is considered the main meal of the day and generally contains rice, bread and vegetables, and sometimes meat (Madanat et al., 2008). Visitors and friends are traditionally offered mansaf which is a sign of respect and generosity to guests throughout Jordan (MOH, 2012). Mansaf is the national dish of Jordan, and it is made of lamb, rice and dried yoghurt, and it is high in fats and carbohydrates ( $\mathrm{MOH}, 2012)$. These high fat and carbohydrate contents make mansaf unsuitable for patients with T2DM. A crosssectional study was conducted at a university hospital to evaluate the dietary patterns among a convenience sample of 750 patients with T2DM (Bawadi et al., 2014). The study results found cereals such as rice and wheat were the main components of the daily diets among patients with T2DM. High consumption of fruits and vegetables were also found, and sodium intake was high in the form of added salt among patients with T2DM. Furthermore, simple sugar and carbohydrate intakes were high, and most of the consumed sugar was in the form of added sugar (Bawadi et al., 2014). Female participants $(n=459,61.2 \%)$ were more represented in the study which may limit generalisation of this study findings. Also, the study was conducted in a teaching hospital, which might not be representative of other hospitals in Jordan, where patients receive medical advice from university students of different specialities.

These results were similar to the dietary habits among the general population in Jordan (Bawadi et al., 2014) since Jordan has witnessed a nutrition transition towards a diet high in sugar, fat 
and refined carbohydrates over the past 40 years (Madanat et al., 2008). As the studies by Khattab et al. (2010) and Al-Amer et al. (2011), which have previously been reported, found that most of the people with T2DM did not also follow the diabetic diet as recommended by the dietitian (Khattab et al., 2010; Al- Amer et al., 2011). Nutrition transition and changes in dietary habits in Jordan might be associated with the high intake of sugar, salt and carbohydrates among patients with T2DM (Bawadi et al., 2014).

People in Jordan insist on consuming honey because of the cultural and religious values of these foods. Honey is mentioned in the Holy Qur'an as blessing foods and source of healing (Purbafrani, Hashemi, Bayyenat, Moghaddam, \& Saeidi, 2014). People believe drinking honey daily can protect them from illness and can be a cure for diseases. Honey is one of the main ingredients in Islamic medicine, and it is used as medicine to treat some patients (Purbafrani et al., 2014). Honey contains ingredients such as hydrogen peroxide (H2O2) and Nitric oxide (NO) which play an important role in the wound healing process (Alam, Islam, Gan, \& Khalil, 2014). A cross-sectional survey in Saudi Arabia reported that honey was the most popular complementary and alternative medicine (CAM) in the treatment of diabetic foot disorders among people with diabetes. The findings reported that $56.6 \%$ of a convenience sample of 346 people with diabetic foot disorders used honey either alone or in combination with other CAM products (Bakhotmah \& Alzahrani, 2010).

A review of literature in neighbouring countries (Al-Saeedi, Elzubier, Al-Dawood, \& Bahnasi, 2002; Khatib, Efrat, \& Deeb, 2007) which share similar cultural norms with Jordan showed that people with diabetes believed that honey does not affect blood glucose levels. Honey has a high glycaemic index (GI) value which is absorbed rapidly increasing blood glucose levels (Hillson, 2015).

However, a cross-sectional study with a randomly selected sample of 1039 conducted in different primary health care centres in Saudi Arabia sought to explore dietary misconceptions among diabetic patients (all types). The survey findings revealed that $34.3 \%$ and $17 \%$ of the participants believed that consumption of honey and dates respectively is beneficial for patients with diabetes (Al-Saeedi et al., 2002). Khatib et al. (2007) also concluded in their crosssectional survey that $56 \%$ of the 150 Arab Israeli people with diabetes in their convenience sample believed that consumption of honey had no impact on their blood glucose levels. The reasons behind the consumption of honey in Saeedi (2002)'s and Khatib et al. (2007)' studies were attributed to cultural and religious factors in Arab society. 
Evidence shows that the Jordanian diet has altered to be low in fruits and vegetables, and high in fats and sugar, and this alteration in Jordanian diet is likely associated with the rise in noncommunicable conditions such as T2DM and cardiovascular diseases (MOH, 2012; Al Nsour et al., 2012; El-Qudah, 2016). Arab Middle East countries including Jordan have witnessed this nutritional transition in food choices from the Mediterranean diet to the fast food pattern during the past 40 years (Takruri \& Alkurd, 2014; El-Qudah, 2016).

Studies show sugar intake is also high among people living in Jordan (Madanat et al., 2008; Al-Khawaldeh et al., 2012; Bawadi et al., 2014; Sharara et al., 2018). Total fat intake rose from $87 \mathrm{~g}$ per person in 2006/2007 to $100 \mathrm{~g}$ per person in 2010 (Takruri \& Alkurd, 2014). The daily intake of energy also increased from $3031 \mathrm{Kcal} /$ day per capita in 2006/2007 to $3325 \mathrm{Kcal} /$ day per capita in 2010 in Jordan (Takruri \& Alkurd, 2014). These figures show there has been a significant alteration in the Jordanian diet to be low in fruits and vegetables, and high in energy, fat, cholesterol, sugar and salt which are considered as the main characteristics of 'Western' diet (Takruri \& Alkurd, 2014: 120). Furthermore, the increasing number of fast food restaurants across Jordan leads to an increase in the availability of fast foods. Fast food, cakes, cookies, pies and fried foods contain trans fats which can lead to high levels of blood cholesterol and contribute to weight gain (Unger, 2012; Al Nsour et al., 2012). Weight gain and hyperlipidaemia are considered the main risk factors for diabetes and diabetes-related complications (ADA, 2015b). Abdel-Aal et al. (2008) reported in their cross-sectional study that $90 \%$ of a convenience sample of 702 patients with T2DM had one or more types of dyslipidaemia in Jordan. This study was conducted in the National Diabetes Centre, and the study participants were more likely to have severe diabetes and complications. Therefore, there may be an overestimation of the prevalence of dyslipidaemia among the study patients which may not reflect all patients with T2DM in Jordan.

In addition to diet, another diabetes self-management behaviour, which has been studied and reported in the literature, is the physical activity, which is now presented and discussed.

\subsubsection{Physical activity}

Physical activity has benefits for people with T2DM (WHO, 2016a). For instance, physical activity may reduce blood glucose levels and the risk of developing diabetes-related complications such as cardiovascular diseases (ADA, 2016). Furthermore, physical activity helps the body cells to use insulin effectively, increases the amount of glucose utilised by muscles and helps patients to lose weight (Hillson, 2015). In contrast, physical inactivity is 
considered as to be the fourth risk factor for global mortality in 2009 (WHO, 2009a). The World Health Organisation (2016a) reported that the Eastern Mediterranean Region, including Jordan, had the highest prevalence of physical inactivity among the World regions in 2010.

The recommended physical activity guidelines for people with diabetes are 30 minutes of moderate to vigorous intensity exercise per day on five days a week, and muscle-strengthening exercises on two or more days a week (WHO, 2016a; ADA, 2015c). Moderate exercise means a person can be able to talk during activity and vigorous exercise implies a person cannot say more than a few words without pausing for a breath during the exercise (ADA, 2015c). People with diabetes should seek advice from health care providers and sports experts before starting any physical activities. Unplanned physical exercise may cause exercise-induced hypoglycaemia especially among people with diabetes who take sulfonylureas or insulin in the management of their diabetes (Hillson, 2015).

The rapid changes in physical activity and food habits have led to the coexistence of many nutritional problems that underlies many chronic conditions such as T2DM in Jordan (ElQudah, 2016). A review of Jordanian literature found that a rise in the prevalence of noncommunicable conditions in Jordan is attributed to high levels of physical inactivity and a sedentary lifestyle (Gallagher, Gebhard, Nash, Occhipinti, \& Walke, 2008). The features of a sedentary lifestyle such as watching television, using a computer, sitting at work and modern means of transportation have led to increasing levels of physical inactivity among people in Jordan (MOH, 2012). People in Jordan perceive physical activity and joining a gym as leisure and secondary activities. Physical exercise is not considered to be a priority within Jordanians' daily routine activities (Gallagher et al., 2008). According to the findings of a cross-sectional survey of a randomly selected sample of 3196 men and women who were all aged $\geq 18$ years, $12.5 \%$ were physically active and $55.9 \%$ of the participants recognised the recommended physical activity guidelines in Jordan (Barghouti, Jaghbir, AbuRmaileh, Jallad, \& Abd- Qudah, 2015).

Despite the publication of research findings that have shown the importance of physical activity, the recommended physical activity among patients with diabetes in Jordan is still low. Low levels of physical activity are reported as being common among patients with poorly controlled diabetes (Khattab et al., 2010; Al- Khawaldeh et al., 2012; Darawad et al., 2016). Darawad et al. (2016) conducted a cross-sectional study which explored exercise behaviours among a convenience sample of 115 patients with both types (Type 1\& 2) diabetes in Jordan. 
The findings revealed that $51(45.1 \%)$ patients reported walking as the most common exercise and lack of desire, time and having diabetes were reasons for not exercising (Darawad et al., 2016). The study findings claimed that the advanced age of the participants hindered their ability to exercise. However, this claim is inaccurate in this study as the mean age of participants was 43.2 years. The use of self-reported measures may be leading to recall bias and data on exercise were also collected only once and may not reflect long-term adherence to the recommended physical activity.

A previously reported cross-sectional study conducted by Khattab et al. in 2010 to identify the factors associated with poorly controlled T2DM in Jordan also found that poor adherence to recommended physical activity guidelines be associated with poor glycaemic control among patients with T2DM. $32.1 \%$ of the 917 patients with T2DM achieved the recommended physical activity guidelines for patients with T2DM (Khattab et al., 2010). The recommended physical activity in this study referred to people with T2DM who walked at least 30 minutes $\geq 3$ days in the last seven days. However, walking alone is not sufficient to meet WHO recommended physical activity levels and this study did not provide information about the reasons for using this criterion and also the intensity of physical exercise. The data about physical exercise were also collected by a yes or no answer and based on self-report which might be subject to recall bias. Furthermore, the exclusion criteria in this study were not fully described, which might include people with cognitive impairment having difficulty in remembering their physical activities in the previous seven days.

Social and cultural norms influence patients with diabetes to be able to do the recommended physical activity. Women face disapproval from society to join health clubs or to exercise in public places (Barghouti et al., 2015). Therefore, females with T2DM have to find other exercise options that they could engage in. Socio-cultural barriers restricted the women to engage in outdoor physical activities such as jogging and running. A previously reported Jordanian survey of 1647 men and 1549 women identified $16.2 \%$ of men, and 8.6\% of women were physically active (Barghout et al., 2015). Low levels of physical activity among women were attributed to barriers such as social and cultural norms and caring for children or older people (Barghout et al., 2015). Omani women with T2DM were also less likely to exercise than men (Alrahbi, 2014). Additionally, most of the sports facilities in Jordan are accessible to men only, and a few sports clubs allow limited times for women to exercise. This issue is also considered as a barrier for women in other Arabic countries (Al-Kaabi et al., 2009; Badran \& Laher, 2012; Alrahbi, 2014). 
Sulaiman et al. (2018) conducted a cross-sectional survey to identify the prevalence of T2DM and physical activity among a randomly selected sample of 872 participants and 204 of them had T2DM in the UAE. Physical activity was defined according to American Diabetes Association guidelines (2015) previously reported in this section. The study findings revealed that $9.8 \%$ of the 204 people with T2DM were physically active according to the American Diabetes Association guidelines (Sulaiman et al., 2018). This study focused only on the frequency of physical activity and did not provide any data about the reasons for the low level of physical activity among patients with T2DM. Al-Kaabi et al. (2009) in their cross-sectional survey of a randomly selected sample of 390 patients with T2DM found that having another disease, lack of time, cultural issues especially for women, lack of interest, family responsibilities, lack of knowledge and experience were considered as barriers to doing exercise in the UAE (Al-Kaabi et al., 2009).

The literature would suggest that patients with diabetes also believe that adherence to recommended physical activity is less important than adherence to medications. Previous studies in Jordan and other Arab countries reported that people with diabetes showed more adherence to medications than physical activity (Khattab et al., 2010; Al-Khawaldeh et al., 2012; Sabbah \& Al-Shehri, 2014). T2DM requires an application of all diabetes management behaviours to control blood glucose levels and delay or prevent complications. Adherence to medications is one of these behaviours, and it is discussed now.

\subsubsection{Adherence to medications}

$\mathrm{T} 2 \mathrm{DM}$ is a complex condition requiring an application of several management treatments and behaviours. Patients have to be able to cope with these diabetes management behaviours over a long period. Taking medications at the scheduled time and the right dose is considered an essential part of diabetes management behaviours (IDF, 2017). Adherence to the medication regimen is challenging for people with diabetes, especially for people who have more than one chronic condition such as diabetes and hypertension. The World Health Organisation and Sabatâe (2003, p. 3) defines adherence as 'the extent to which a person's behaviour - taking medication, following a diet, and/or executing lifestyle changes, corresponds with agreed recommendations from a health care provider.' Taking multiple pharmacological treatments and regular dosages may negatively influence patient's adherence to T2DM medication regimen (Antoine, Pieper, Mathes, \& Eikermann, 2014; Jarab, Mukattash, Al-Azayzih, \& Khdour, 2018). 
Diabetic pharmacological treatments consist of oral hypoglycaemic agents and insulin. Oral hypoglycaemic agents such as metformin, sulfonylureas and thiazolidinedione are only effective in the presence of insulin in the body (Hillson, 2015). Metformin is usually considered as a first drug choice and a cornerstone medication in the management of T2DM (Unger, 2012). When metformin is unable to control glycaemic levels, the second agent such as sulfonylureas is added. If glycaemic levels remain poorly controlled, third agents such as thiazolidinedione or insulin should be added (IDF, 2017).

Insulin is a hormone that is produced in the pancreas in response to high blood glucose levels. Insulin helps the body to use or to store the glucose that is gained from food (ADA, 2015a). Frederick Banting was the first person who introduced insulin to be used to control diabetes in humans (IDF, 2013). The body has to produce insulin, but insulin therapy is not usually the first course of action in treating T2DM (IDF, 2013). Most people with T2DM consider adding insulin therapy to their treatment regimen as an indicator of the unsatisfactory progress of the disease (Unger, 2012). The complexity of insulin therapy, the sign of poor progress and negative beliefs towards insulin are associated with psychological insulin resistance phenomenon (Holmes-Truscott, Blackberry, O’Neal, Furler, \& Speight, 2016). This phenomenon was also identified among people with poorly controlled T2DM in Jordan as illustrated by Khattab et al. (2010) who found that the high prevalence of poorly controlled T2DM among patients who were taking a combination therapy of oral hypoglycaemic agents and insulin contributed to the delay in adding insulin to the medication regimen by doctors in Jordan. Therefore, these patients associated insulin therapy with a severe stage of diabetes and the development of complications. Patients' perceptions of insulin therapy are supported by another cross-sectional study conducted with a convenience sample of 223 patients with T2DM (Al-Khawaldeh et al., 2012). This study's findings found that patients perceived insulin therapy as an indicator of poor glycaemic control. This perception was attributed to more than half of the patients who used insulin, who had a $\mathrm{HbA1c}$ greater than 7 and insulin was added as a result of high blood glucose levels among patients.

Adherence to pharmacological treatment is influenced by several factors. Antoine et al. (2014) reported that the ability of people with T2DM to follow their medication regimen was compromised by multiple barriers such as long-term multi-therapies, adverse effects of medications and patients not understanding the information given by health care providers. A qualitative study was conducted with 36 patients with T2DM recruited by a purposeful sampling approach from an outpatient diabetes clinic in Jordan (Jarab et al., 2018). Focus group 
method was used to collect data from participants in the study. This study's findings showed that concerns about side effects of the medications, lack of confidence in the effectiveness of diabetes medications and fear from insulin injections affected participants' adherence to the medication regimen (Jarab et al., 2018).

Arifulla, John, Sreedharan, Muttappallymyalil and Basha (2014) conducted a cross-sectional survey to identify adherence rate to medications among a purposive sample of 132 patients with T2DM in the United Arab Emirates. The findings reported that forgetfulness, lack of finance, interfering with patients' meal plans and multiple medications were the main barriers to adherence to the medication regimen. The subjective nature of the data on medication adherence in this study which may be influenced by recall bias. Also, the self-reported adherence rate was not confirmed with blood glucose levels or HbA1c values.

This study's findings were consistent with another cross-sectional survey conducted in Jordan by Awwad, Akour, Al-Muhaissen and Morisky (2015) who revealed that $38.4 \%$ of the 902 people with chronic conditions including hypertension, asthma, osteoporosis, diabetes mellitus and chronic kidney disease in their convenience sample reported forgetfulness was the main barrier to adherence to the medication regimen and $13.1 \%$ stated that they did not like taking medications. Therefore, providing a medication reminder is considered one of the effective methods to improve diabetes management behaviours among people with diabetes (WHO, 2016a). This study was based on self-reported measures, so it is liable to recall bias. Also, adherence to medications might be subjected to the influence of a socially desirable answer, especially as this study was a face-to-face structured interview.

In addition to forgetfulness and aversion, adverse effects of medications and multiple medications were other main barriers to adherence to the medication regimen in this study. Furthermore, another cross-sectional survey also reported that difficulties with having prescription refills on time, multiple medications, adverse effects of medications and negative beliefs were the main factors associated with poor adherence rate (Basheti, El Hait, Qunaibi, Aburuz, \& Bulatova, 2016). 53.9\% of a random sample of 167 of people with chronic conditions including cancer and diabetes mellitus were adherent to medications, and people with diabetes had the lowest adherence rate among all patients with chronic conditions in Jordan (Basheti et al., 2016). Data on adherence to medications were collected only once during the year in this study and may not reflect long-term adherence to medications. 
The literature would suggest that Jordanian patients with diabetes believe that adherence to medications is enough to manage their condition (Khattab et al., 2010; Al-Amer et al., 2011; Al-Khawaldeh et al., 2012; Albikawi \& Abuadas, 2015). According to people with diabetes, adherence to medications is more important than other diabetes management behaviours such as diet, physical activity and monitoring blood glucose levels. The Jordanian literature showed that adherence to medications was high among people with diabetes in Jordan compared with other diabetes management behaviours (Khattab et al., 2010; Al-Amer et al., 2011; AlKhawaldeh et al., 2012; Albikawi \& Abuadas, 2015). Khattab et al. (2010) used a questionnaire to study diabetes self-management behaviours among 917 patients with T2DM in the previous seven days. The study results illustrated that $91.9 \%$ of participants were adherent to medication regimen compared with $38.1 \%$ who monitored blood glucose levels at home, $18.6 \%$ followed a diabetic diet and $32.1 \%$ of participants engaged in physical activity. These figures reflect a high level of adherence to medications among patients and indicate patients' preference to adhere to behaviours that require the least effort and are less challenging.

Other researchers (Al-Khawaldeh et al., 2012; Albikawi \& Abuadas, 2015) explored the reasons behind the highest adherence rate of taking medications among all diabetes management behaviours. Al-Khawaldeh et al. (2012) found in their cross-sectional survey with a convenience sample of 223 patients with T2DM that high adherence to the medication regimen among patients was attributed to patients' perceptions towards diabetes management and potential barriers to adhering to other diabetes management behaviours such as social, cultural, financial and medical factors that were not measured in this study. Furthermore, Albikawi and Abuadas (2015) found in their cross-sectional survey with 149 patients with T2DM recruited by a convenience sampling that lifestyle modification was the main barrier to adhere to other T2DM management behaviours such as diet and exercise, foot care and blood glucose monitoring in Jordan. The data regarding adherence to medications were based on a self-report which might be subject to recall bias. Also, patients were overestimating their adherence to medication or the method used to assess medication adherence might be subjected to the influence of a socially desirable answer leading to unreliable results.

Pharmacological agents such as oral hypoglycaemic agents and insulin are adjusted to blood glucose levels obtained via finger prick. Monitoring blood glucose is a skill that each person with diabetes should be able to accomplish (Hillson, 2015). The literature which has explored this aspect of diabetes management behaviour is reviewed now. 


\subsubsection{Monitoring blood glucose}

Glycaemic control is essential to monitor diabetes management and tight glycaemic control has been shown to decrease the rates of diabetes-related complications (Diabetes UK, 2013). Selfmonitoring blood glucose (SMBG) is an important component of diabetes management behaviours that enables people with diabetes to evaluate their blood glucose level, interpret glucose readings and adjust their treatment and lifestyle (Hillson, 2015). SMBG should be part of daily routine among people with T2DM who manage their diabetes through insulin or sulfonylureas due to dosage adjustment and avoiding hypoglycaemia (Diabetes UK, 2013; Hillson, 2015). However, before incorporating SMBG into patients' diabetes care plan, training is required, and only patients who have completed formal training sessions can monitor their blood glucose at home (Hillson, 2015).

The American Diabetes Association (2018) suggest that an SMBG education programme should include multiple components, for instance, the correct technique of doing SMBG, reading and interpreting the results, adjusting diabetes management behaviours based on glucose readings, and proper documenting and reporting the results to the health care providers. Education should also contain "motivational knowledge", that empowers patients to take part in diabetes management and shows the importance of good glucose control (Diabetes UK 2013, p. 6).

Jordanian studies (Khattab et al., 2010; Al-Amer et al., 2011) have assessed adherence to SMBG based on general and unexplained criteria. Adherence to SMBG referred to people with diabetes who measured their blood glucose level at home $\geq$ five days in the last seven days (Khattab et al., 2010; Al-Amer et al., 2011). This criterion was not based on prescribed or recommended patterns of SMBG for each individual with diabetes. There is also no evidence regarding the optimal frequency of SMBG, and the level of adherence to prescribed SMBG varies from individual to another and what type of treatment that the patient takes (ADA, 2013). For instance, patients with T2DM who are on diet or metformin alone with good controlled diabetes do not need to do SMBG on a daily basis (Hillson, 2015).

Khattab et al. (2010) conducted a cross-sectional study to identify the factors associated with poorly controlled T2DM in Jordan. The study results illustrated that $61.9 \%$ of the 917 patients with T2DM did not monitor their blood glucose at home and poorly controlled T2DM was also high among them. Due to unclear criteria as to whether routinely collected SMBG was required, it could be argued that as $62 \%$ of participants in the sample were on oral 
hypoglycaemic agents alone, that criteria of an SMBG at home $\geq 5$ days in the last seven days might not apply to them since non-insulin-treated T2DM patients do not need to measure SMBG routinely (Hillson, 2015). Also, there is no recommendation for the frequency or the timing of the measuring BG levels as the evidence is still insufficient (ADA, 2018).

This result is similar to another cross-sectional study with a systematic random sampling of 649 patients with diabetes (90\% of them had T2DM) in Jordan which showed that $24 \%$ of participants measured their blood glucose at home for five days or more in the previous seven days (Al-Amer et al., 2011). The reason for using this criterion was not justified in these studies. Furthermore, the data regarding SMBG was collected by yes or no answer and based on a selfreport rather than actual measurements, which might be subject to recall bias.

Adherence to SMBG includes correct performance and frequency of SMBG. Studies in Jordan (Khattab et al., 2010; Al-Amer et al., 2011; Albikawi \& Abuadas, 2015) focused only on frequency and did not assess the performance. These studies did not mention whether the participants received proper training about SMBG or not, which is considered as a cornerstone, before SMBG is practised by people with diabetes (Hillson, 2015).

Albikawi and Abuadas (2015) found that SMBG was the least performed behaviour among the diabetes management behaviours and low motivation was the main barrier to SMBG regimen. This cross-sectional survey used more specific criteria than the previous studies (Khattab et al., 2010; Al-Amer et al., 2011) to asses SMBG among 149 patients with T2DM recruited by a convenience sampling from a Diabetes Specialized Centre. Patients were asked to report monitoring glucose levels as prescribed by their health care provider in the previous seven days. $11 \%(n=17)$ of the participants performed SMBG in accordance with what their health provider prescribed. Furthermore, Al- Maskaril et al. (2013) aimed to identify the main barriers associated with SMBG among 575 people with diabetes randomly selected from two teaching hospitals in the United Arab Emirates. The cost, being a waste of time, lack of knowledge about reading results and being painful were reported as the main barriers to adhering to the SMBG regimen.

A cross-sectional survey went beyond focusing on the prevalence of SMBG to identify SMBG attitude and practice among 1079 patients with diabetes (Type $1 \& 2$ ) recruited by convenience sampling from 18 hospitals and health care centres in Jordan (Al-Keilani, Almomani, AlSawalha, \& Shhabat, 2017). Of all participants, 59\% ( $\mathrm{n}=636)$ were SMBG adherent and patients who received health education about SMBG showed higher SMBG adherence. This 
study also found that patients on oral hypoglycaemic tablets and/or insulin therapy were more likely to adhere to SMBG as compared to those on diet or lifestyle modifications (Al-Keilani et al., 2017). This study reported that half of the participants $(n=548)$ received proper training about SMBG. However, the data regarding frequency and reasons for doing SMBG were collected by self-report which might be subject to recall bias. Also, the reason for using the criterion to assess adherence to SMBG was not justified in this study. Patients were asked to report the frequency of monitoring glucose levels (daily, weekly, monthly and occasionally).

In addition to the previous diabetes management behaviours, foot care is also important as poorly controlled diabetes can lead to diabetic foot complications. The literature on diabetic foot care is reviewed now.

\subsubsection{Foot care}

Diabetic foot complications easily develop among patients who suffer from peripheral arterial disease (poor blood supply) or diabetic neuropathy which reduces the feeling of pain, temperature, touch and position of feet, or both (Hillson, 2015). Diabetic foot problems such as amputation, infection and foot ulceration are considered as the significant causes of disability and morbidity among patients with diabetes (ADA, 2013). Foot complications also have a financial impact on the health care system; for instance, $£ 650$ million was spent on the management of diabetic foot problems in the United Kingdom in 2012 (National Institute for Health and Care Excellence, 2015). However, diabetic foot problems can be prevented or delayed by foot care education, well-controlled diabetes and regular self-monitoring of foot health (ADA, 2013). In addition to other diabetes management behaviours, adherence to foot hygiene, proper footwear and foot care on a daily basis at home are important activities among patients with diabetes (WHO, 2016a). A small blister on the toe of a patient with diabetes may lead to leg amputation and long-term disability (Hillson, 2015; Khawaja et al., 2018). This is illustrated in the following prospective cohort study in Jordan which showed how foot infection progressed to leg amputation.

Only one longitudinal cohort study was identified which had followed up 60 patients with a diabetic foot infection between 2005 and 2007 to identify the progression of diabetic foot infection (Salayta \& Aleyadeh, 2010). Diabetic foot treatment protocol in the selected study setting consisted of three phases of interventions. The first phase of treatment begun with debridement and when debridement failed, the amputation of one or more toes was considered. When infection remained uncontrolled, a below-knee amputation procedure was needed to 
eradicate the infection. The results showed that $17 \%(n=10)$ of participants had below- knee amputation which was not linked to the duration of diabetes or type of treatment. It was associated with neuropathy in which $70 \%(n=41)$ of the study participants had neuropathy. Patients with neuropathy are asymptomatic which means that they are prone to insensate foot complications (Khawaja et al., 2018). The sample size in this study was small and conducted in only one study setting. Sampling methods and factors such as glycaemic levels and adherence to diabetes management behaviours were also not explored in this study. However, this study added valuable results to the Jordanian literature regarding the prevalence of leg amputation. The high prevalence of below-knee amputation might be due to a lack of patient education and lack of a multidisciplinary diabetes specialist foot care team in the study setting. Incorporating an interdisciplinary team in the management of diabetic foot has been shown to reduce the incidence of leg amputation (Hillson, 2015).

Diabetic neuropathy is considered the leading risk factor in the development of diabetic foot problems (WHO, 2016a). A cross-sectional study was conducted to estimate the prevalence rate of diabetic neuropathy among 1003 patients with T2DM in Jordan recruited by convenience sampling from the National Diabetes Centre (Khawaja et al., 2018). The overall prevalence of diabetic neuropathy was $39.5 \%$ out of the 1003 patients, and $57.9 \%$ of the patients $(\mathrm{n}=581)$ had a HbA1c more than 7\% (Khawaja et al., 2018). The study participants were recruited from the National Diabetes Centre and not from a community setting. The National Centre is not accessible to all people, especially those who live in rural areas, which may limit the generalisation of the study findings.

Bakri, Allan, Khader, Younes and Ajlouni (2012) also recruited a large sample size of a randomly selected sample of 1000 patients with diabetes to identify the prevalence rate of diabetes foot complications in Jordan. The findings of this cross-sectional survey illustrated that the prevalence of foot ulcers was $4.6 \%$, neuropathy $14.9 \%$, ischemia $7.5 \%$ and amputation $1.7 \%$. Poorly controlled diabetes and obesity were high $77 \%$ and $50 \%$ among participants respectively. However, the study findings were not linked to poor glycaemic levels or obesity. The findings were mainly associated with duration of diabetes, neuropathy and male gender. Longer duration of diabetes was also found as a significant factor in the development of diabetic foot disorder among patients with diabetes in Saudi Arabia (Alzahrani, Wang, Alzahrani, \& $\mathrm{Hu}, 2015)$. 
Al-Khawaldeh et al. (2012) and Albikawi and Abuadas (2015) in their cross-sectional surveys found that feet washing was the most performed foot care behaviour and inspecting the inside of shoes was the least performed among patients with T2DM in Jordan. The high adherence to feet washing was attributed to Muslim participants who have to wash their feet at least once a day as a religious practice before prayer (Albikawi \& Abuadas, 2015). A diabetes self-care activities scale was used in these cross-sectional studies. Participants were asked to report the number of days of performing foot care behaviour in the previous seven days (Al-Khawaldeh et al., 2012; Albikawi \& Abuadas, 2015). The data regarding adherence to foot care were based on a self-report which might be subject to recall bias.

A qualitative study was conducted with seven patients with diabetes recruited by a purposeful sampling approach from six hospitals in Jordan (Abu-Qamar \& Wilson, 2011). Unstructured interview method was used to collect data from participants in the study. This study found that health promotion and prevention regarding foot health are not addressed effectively in the health care system in Jordan, which mainly focuses on management of acute diabetic foot problems (Abu-Qamar \& Wilson, 2011). For instance, health care providers did not examine the feet of a person with diabetes on a regular basis in Jordan (Abu-Qamar \& Wilson, 2011; Albikawi \& Abuadas, 2015). These findings are in accordance with other findings in Saudi Arabia and the United Arab Emirates (Al Odhayani, Tayel, \& Al-Madi, 2017; Al-Kaabi, Al Maskari, Cragg, Afandi, \& Souid, 2015). 20.6\% of 350 patients with diabetic foot complications reported that they never had their feet examined by health care providers in Saudi Arabia (Al Odhayani et al., 2017). A cross-sectional study with 422 patients with diabetes aimed to identify foot care and problems in the UAE (Al-Kaabi et al., 2015). The findings showed that $20 \%(n=83)$ of the participants reported receiving formal foot care training (Al-Kaabi et al., 2015). Both of the studies were cross-sectional surveys and based on a self-report which might be subject to recall bias.

There is a scarcity of data regarding the availability of necessary diabetic foot examination procedures such as foot vibration perception by tuning fork and foot vascular status by Doppler in the primary care settings in Jordan (WHO, 2016b). If these basic procedures are not available in rural areas in Jordan, patients have to travel to hospitals to seek foot examination or not have their feet examined which may result in a delay in seeking foot care (Abu-Qamar \& Wilson, 2011). Furthermore, participants did not practice preventive foot care behaviours as they believed that regular self-monitoring of the foot was not an element of diabetes management behaviours unless a foot ulcer was present (Abu-Qamar \& Wilson, 2011; Albikawi \& Abuadas, 
2015). Foot care was not seen as a preventive measure, but only something to be practised once a problem had occurred.

A cross-sectional survey in Saudi Arabia reported that honey was the most popular complementary and alternative medicine (CAM) in the treatment of diabetic foot disorders among patients with diabetes. These survey findings indicated that $56.6 \%$ of 346 people with diabetic foot complications used honey either alone or in combination with other CAM products in the treatment of diabetic foot complications (Bakhotmah \& Alzahrani, 2010). The healing properties of honey such as being an antimicrobial, antioxidant and anti-inflammatory have been shown to improve the wound healing process (Al-Hariri, 2013). However, only approved honey by the local authority should be used in the treatment of diabetic foot wounds because using commercially produced honey may lead to purchasing diluted processed honey which lacks natural components (Alam et al., 2014).

Having reviewed the literature, which has explored diabetes self-management behaviours, a further theme derived from the literature related to the attitudes, knowledge and perceptions of patients of T2DM regarding their condition which is now presented and discussed.

\subsection{Attitudes, knowledge and perceptions of patients with Type-2 diabetes regarding diabetes}

Knowledge of T2DM is one of the important elements in a diabetes management plan. Knowledge about diabetes includes many aspects such as knowledge about causes, complications, healthy behaviours, medications and diet (Hillson, 2015). Good diabetes knowledge may facilitate adaptation of healthy behaviours and improve patients' adherence to their diabetes management plans. For instance, patients with T2DM should be able to monitor and interpret blood glucose readings accurately. This may help to reduce acute complications such as diabetic ketoacidosis (Unger, 2012).

Several studies have shown that diabetes knowledge is important to change management behaviours among patients in Jordan (Al-Sarihin et al., 2012, Al-Khawaldeh et al., 2012; Adwan \& Najjar, 2013; El-Qudah, 2016; Jarab et al., 2018). However, knowledge alone is not enough to change behaviours, and it should be complemented by behaviours and positive attitudes towards diabetes and the treatment plan (Naidoo \& Wills 2009).

In Jordanian literature, most of the researchers evaluated either diabetes knowledge (Al-Sarihin et al., 2012; Habashneh, Khader, Hammad, \& Almuradi, 2010) or patients' behaviours 
(Albikawi \& Abuadas, 2015) alone, which is identified as a shortfall. Knowledge, attitudes and behaviours are required to be explored together to provide a holistic understanding of the reasons behind poorly controlled T2DM.

Al-Khawaldeh et al. (2012) found in their cross-sectional survey with a convenience sample of 223 patients with T2DM that poorly controlled T2DM was high among insulin users which might be attributed to limited knowledge or abilities or skills. Another cross-sectional study aimed to identify the relationship between glycaemic control and education among 164 patients with T2DM in Turkey (Ozcelik et al., 2010). The study results revealed that HbA1c and knowledge levels were better among patients who received diabetes education than patients who did not receive an education. The nature and content of the health education programme were not explained in this study.

A review of the literature also showed patients with diabetes often score poorly on diabetes knowledge tests in Jordan (Al-Sarihin et al., 2012; Habashneh et al., 2010). In a cross-sectional study of 100 diabetic patients recruited by convenience sampling from one military medical centre, the average score was $49.8 \pm 13.4 \%$ using Fitzgerald's (1998) questionnaire to assess general diabetes knowledge and insulin knowledge (Al-Sarihin et al., 2012). This questionnaire consists of 23 questions, and the results are categorised as: less than 52\% is considered as poor, $52-78 \%$ is an average, and greater than $78 \%$ is good knowledge. However, the author did not identify the main gaps in knowledge among participants. This study included patients who were aged over 18 years and approximately three-quarter of the participants were aged over 51 years. However, there was no statistically significant differences between different age groups of the participants in both general diabetes knowledge test and insulin knowledge test (AlSarihin et al., 2012). The low levels of knowledge in this study were attributed to the lack of a diabetes education programme which is essential in improving diabetes knowledge among patients. Additionally, low literacy rates were identified due to approximately $80 \%(n=81)$ of the participants only completing up to secondary school education (Al-Sarihin et al., 2012). Knowledge deficit in this study suggested the need for a diabetes education programme.

There are also other factors which may attribute to low levels of knowledge among patients with diabetes. Several studies reported that low literacy rates, low family income and low selfcare were the factors which contribute to poor knowledge levels among patients with diabetes (Al-Sarihin et al., 2012; Al-Adsani, Moussa, Al-Jasem, Abdella, \& Al-Hamad, 2009). Although, the sample size was small (7 participants), Abu-Qamar and Wilson (2011) 
highlighted that patients with diabetes sought their information about diabetes from family members or friends rather than health care professionals, which may have contributed to poor knowledge levels among them.

The family member with diabetes may be considered as a good source of information. Good support and a positive relationship with health care professionals also have been shown to have a positive impact on levels of knowledge among patients (Al-Sarihin et al., 2012; Al-Qazaz, Hassali, Shafie, Sulaiman, \& Sundram, 2011). A cross-sectional study aimed to evaluate the awareness of diabetic retinopathy among a sample of 237 patients with T2DM randomly selected from three main cities in Jordan (Bakkar et al., 2017). This study reported that $88.2 \%$ $(n=209)$ of the participants were aware that diabetes could affect the retina and $81.9 \%(n=194)$ patients mentioned that diabetic retinopathy could lead to blindness. Despite the high level of awareness about diabetic retinopathy in the study, the level of patients' compliance with behaviours to reduce the risk of retinopathy was low, with a total of $29.5 \%$ of participants having had an eye examination in the previous year (Bakkar et al., 2017). Therefore, knowledge alone was not enough to stimulate and trigger the patients to take action and change their habits. This study's findings also reported that general practitioners, interpersonal relationships and the mass media were the main sources of information about diabetes among the study sample (Bakkar et al., 2017).

Furthermore, a longer duration of T2DM and attending diabetes education were associated with good levels of knowledge of diabetes (Al-Adsani et al., 2009; Ozcelik et al., 2010). Al-Adsani et al. (2009) conducted a cross-sectional survey to identify the level of diabetes knowledge among 5114 people with T2DM. The Michigan diabetes knowledge test (1998) was used as a tool to collect data which consists of 23 questions. The score is categorised as greater than 17 is good, 11-17 is average and less than 11 is considered as poor knowledge. The results showed that people with a duration of diabetes of more than 20 years had a mean score of $14.2 \pm 3.2$ while the mean score of patients with less than five years of diabetes was $13.1 \pm 3.4$ which is linked to people with T2DM learning and expanding their knowledge and experience with time. The family history of diabetes, poor glycaemic control levels, more family income and having more complications had also contributed to better knowledge of the condition (Al-Adsani et al., 2009). The data were collected based on a self-report which might be subject to recall bias. Also, Kuwait is wealthier than Jordan and has different education provision for patients with T2DM compared with Jordan. These factors may impact the level of knowledge among patients in Kuwait compared with patients' knowledge of diabetes in Jordan. 
Effective management of T2DM needs to combine the implementation of both lifestyle modifications and pharmacological treatment to achieve the target glycaemic goals and prevent further complications (WHO, 2016a). Lifestyle changes and adherence to diabetes treatment plans are influenced by patients' perceptions, health beliefs' and their attitudes towards T2DM (Alsairafi, Taylor, Smith, \& Alattar, 2016). Understanding patients' perceptions of their condition and their attitudes towards their treatment plans is considered an important element of effective diabetes care (IDF, 2012).

Understanding patients' attitudes is essential to provide comprehensive diabetes care to patients. This may enable health care workers to identify patients' attitudes, which may underpin their behaviours. Abolghasemi and Sedaghat (2014) reported that understanding of patients' attitudes is required to understand patients' behaviours and develop effective educational and treatment programmes. Therefore, this can lead to support for the essential components of a patient's health; physical, psychological and social aspects as proposed by the WHO (WHO, 1948).

From reviewing the literature, most of the studies about attitudes of people with diabetes in Jordan and neighbouring countries that share a similar cultural context, used the diabetes attitude questionnaires as the data collection tool (Saadia, Rushdi, Alsheha, Saeed, \& Rajab, 2010; Khattab et al., 2010; Al-Maskari et al., 2013; Asdaq, 2018), which is identified as a shortfall in the literature. Abolghasemi and Sedaghat (2014) also found in their review of Iranian literature that of the 12 studies about attitudes of people with diabetes, 11 studies used quantitative methods and only one study used a qualitative approach (grounded theory). I argue that to gain an in-depth understanding attitudes should be examined through qualitative methods to enable patients to explain the attitudes which underpin their behaviours and perceptions from their perspectives. Patients with T2DM need to have the opportunity to express their feelings and concerns regarding their condition.

Patients with diabetes express different kinds of attitudes towards their condition that may influence their treatment plans. The Diabetes Attitudes, Wishes, and Needs (DAWN) Program that is conducted in 13 different countries in Europe, Asia, Australia and the USA with 5000 patients with diabetes showed, upon diagnosis of diabetes, that $85.2 \%(n=428)$ of patients reported feeling high levels of distress including guilt, anxiety and anger (Skovlund \& Peyrot, 2005). Furthermore, Al-Adsani et al. (2009) conducted a cross-sectional survey to identify the level of knowledge among 5114 people with T2DM in Kuwait. The results showed that people 
with shorter duration of diabetes which was classified as less than five years had poor levels of knowledge due to emotional distress upon diagnosis of diabetes which may hinder them to retaining information. Patients with diabetes have also been reported as being in denial towards their condition which could be a barrier to adapting to a healthy lifestyle (Gazmararian, Ziemer, \& Barnes, 2009).

Patients' attitudes are associated with other factors such as glycaemic control, diabetes knowledge and diabetes-related behaviours. These four factors are connected and should be integral to any diabetes treatment plan. Khattab et al. (2010) found that the negative attitudes towards diabetes among patients were associated with poorly controlled T2DM in Jordan. This result is similar to the findings from a cross-sectional study with 575 patients in the United Arab Emirates which showed that negative attitudes towards diabetes were common among people with poorly controlled diabetes (Al-Maskari et al., 2013). Another cross-sectional study in Saudi Arabia conducted with 570 female patients with diabetes found that $17.5 \%$ of them scored higher than $50 \%$, which is an average score, on an attitude questionnaire. Positive attitudes towards diabetes might be achieved by conducting education and awareness programmes (Saadia et al., 2000). Abolghasemi and Sedaghat (2014) reported patients who had poor diabetes knowledge regarding the causes, complications and treatment of diabetes showed negative attitudes towards diabetes.

A patient's perception of diabetes is a key element in the diabetes treatment plan. Diabetes perception is how the patients identify and understand diabetes and the beliefs that they hold about their condition (Fitzgerald et al., 2008). A cross-sectional study with 177 American patients with poorly controlled diabetes was conducted to study patients' perceptions of diabetes. The findings reported that $23.7 \%$ of patients identified their diabetes control as being good and $39.6 \%$ of participants described their diabetes control as moderate in spite of the average levels of HbA1c among these two groups being 9.5\% and 9.8\% respectively (Gopalan, Moss, Tao, Zhu, \& Volpp, 2014). This low level of awareness was similar to findings from another cross-sectional study with a randomly selected sample of 386 diabetic patients in Saudi Arabia, which showed that $70.7 \%$ of the study participants reported a poor understanding of HbA1c levels and $61.1 \%$ of them were unaware of the type of diabetes they had (Sabbah \& AlShehri, 2014).

A qualitative study with 12 patients with T2DM in Malaysia was conducted to identify patients' perceptions regarding their condition and adherence to medication. Semi-structured 
interviews were used as a data collection method and convenience sample technique was utilised. The findings reported most of the patients were unaware of the type of diabetes they had and doctors did not offer enough information regarding the adverse effects of medication (Al Qazaz et al., 2011). This study was based on a limited sample of qualitative interviews with patients with diabetes attending the Malaysia University Health Clinic. The findings are of direct relevance to the setting in which the study was conducted. However, as the study was conducted at the Malaysia University Health Clinic, this clinic may not be representative of other clinics in Malaysia. In addition, all the interviews were conducted in the English language though all participants were Malaysian, so it may be that other issues and themes might have been identified from patients who cannot communicate in English (Al Qazaz et al., 2011). There are no published qualitative studies identified from Jordan regarding patients' perceptions of their condition.

The health care system and health care workers influence patients' perceptions of diabetes. Al Qazaz et al. (2011) reported that health care providers in Malaysia do not consider and pay attention to patients' perceptions regarding offered advice and information. Furthermore, effective communication between patients and health care providers influence patients' perception that may improve patients' understanding of glycaemic control levels and diabetes management among patients with poorly controlled diabetes (Gopalan et al., 2014).

Having reviewed the literature on the attitudes, knowledge and perceptions of patients towards their condition, a further common issue identified in the literature was the use of complementary medicine which is now reported.

\subsection{Complementary medicine and diabetes}

T2DM is a chronic condition that is managed by multiple approaches. As previously discussed, oral hypoglycaemic agents and insulin therapy are the core elements in the management of T2DM. However, patients tend to use herbal remedies as part of the management of their condition. In the Declaration of Alma-Ata, the WHO (1978) pointed out the importance of incorporating traditional medicine into the primary health care system and primary care services. There are multiple explanations for using complementary medicine among patients such as a primary source of treatment, cultural influences of herbs and used as a complementary treatment (WHO, 2014). Alternative or complementary medicine is a type of medicine that uses natural and herbal remedies in the treatment and management of diseases without adding any artificial ingredients (Alkhazrajy, Habib, \& Alsudani, 2014). 
Many of the current medications are derived from herbs. Plants provide the basic ingredients for making multiple medications (Alkhazrajy et al., 2014; Ahmed, 2016). In 2003, the WHO estimated that $25 \%$ of all medicines were extracted from plants (Helman, 2007). Metformin, a drug that is used to lower blood glucose levels, is derived from a plant called Galega officinalis (Bailey \& Day, 2004). However, herbal use is not only sought for medical reasons, but also represents the cultural heritage of people and the involvement of families and friends in patients' care (Helman, 2007). For instance, the main sources of herbal information identified from the research were the media, family and friends among patients with diabetes in Jordan and the United Arab Emirates (Wazaify, Afifi, El-Khateeb, \& Ajlouni, 2011; Wazaify, Alawwa, Yasein, \& Afifi, 2013). This illustrates the important role of the interpersonal and social factors in patients' health.

There are strong cultural and historical beliefs in complementary or alternative medicine (CAM) to be used in the management of chronic conditions including diabetes in Jordan (Wazaify et al., 2013). Accessibility, affordability and availability of herbs in Jordan encourage patients with diabetes to incorporate herbs into their diabetes management plan (Wazaify et al., 2011). Moreover, herbalism is an indigenous symbol in Jordanian culture (Wazaify et al., 2013). For example, a cross-sectional study was conducted to explore dietary knowledge among a convenience sample of 180 diabetic females in Jordan (El-Qudah, 2016). Approximately half of them $(n=88)$ had a strong belief in the benefits of herbal and traditional medicine in the management of diabetes (El-Qudah, 2016). In addition to the above-mentioned factors, there are several explanations for using herbal medicine in the management of diabetes in Jordan as presented in the following study. Wazaify et al. (2011) conducted a cross-sectional survey to identify the prevalence and the factors associated with herbal use among patients with diabetes in Jordan. The study findings illustrated that $16.6 \%(n=166)$ of a sample of 1000 patients with diabetes were users of herbal remedies in Jordan, and green tea was the most popular herb used by participants. The main reasons for using herbs in this study were attributed to relieving diabetes symptoms, curing diabetes, slowing disease progression and reducing the undesirable side effects of medications. The data were collected based on a selfreport which might be subject to recall bias. Also, the study was conducted in the National Diabetes Centre which might not be representative of other hospitals in Jordan. Also, the National Diabetes Centre is not accessible to all people, especially who live in rural areas, which may limit the generalisation of the study findings. Hoping to cure the disease was also mentioned as a reason behind using herbs among patients in Iraq (Ahmad, 2016). 
People in the Arab World are well influenced by instructions and recommendations mentioned by Prophet Muhammad (the Prophet of Islam) and in Islamic traditions. In addition to the cultural and heritage meaning of herbs, patients tend to use herbs due to religious beliefs. Ginger, olives, black cumin, fenugreek and garlic are examples of herbs mentioned in the Holy Quran and by Prophet Muhammad (Ahmad, 2016). For instance, zingiber officinale (ginger) was the most used herb by people in Kurdistan, Iraq due to the influence of Islamic beliefs on Kurdish society (Ahmad, 2016). Indigenous Arabic and Islamic Medicine incorporate healing practices such as medicinal plants that emerged from; Islamic religion, the medicine of the Prophet (also known as Tibb Nabawi in Arabic) and cultural norms of the inhabitants (Alrawi $\&$ Fetters, 2012). For instance, 26 plants have been used in the management of diabetes mentioned in Arab medicine (Saad, Azaizeh, \& Said, 2005). Examples of the used parts of plants are leaves, flowers, seeds and roots (Ahmad, 2016).

Research about the beneficial features of plants has attracted many researchers worldwide. I focused my review of the literature in relation to herbal use to the herbs most frequently used in Jordan. Furthermore, non-Jordanian studies that evaluated similar herbs used by patients in Jordan were reviewed. Experimental studies in vitro and animals have suggested multiple hypoglycaemic effects of using herbal remedies among patients with diabetes. For example, Abelmoschus esculentus (okra), Allium cepa (onion), Allium sativum (garlic), Cinnamon zeylaniucm blume (cinnamon), Nigella sativa (black cumin), Olea europaeal (olive leaves), Trigonella foenum-graecum (fenugreek) and Teucrium polium (germander) have beneficial properties such as improving insulin secretion, lowering fasting blood glucose, increasing glucose utilisation by body tissues and delaying gastric emptying of carbohydrates after eating (Sinjab \& Youssef, 2014; Ahmad 2016). These herbs are commonly used among patients with diabetes in Jordan (Wazaify et al., 2011; Wazaify et al., 2013). Cinnamon, olives, black cumin, fenugreek and ginger are also the main herbs prescribed by traditional healers to a patient with diabetes in Kurdistan, Iraq (Ahmad, 2016). Table 2 provides examples of herbs that have been used among patients with diabetes in Jordan in previous studies.

\begin{tabular}{|l|l|l|}
\hline English Name & Local Name & Scientific Name \\
\hline cinnamon & Kerfah & $\begin{array}{l}\text { Cinnamon } \\
\text { blume }\end{array}$ \\
\hline fenugreek & Helba & Trigonella foenum-graecum \\
\hline
\end{tabular}




\begin{tabular}{|l|l|l|}
\hline garlic & Thoom & Allium sativum \\
\hline germander & Geádah & Teucrium polium \\
\hline ginger & Zanjabeel & Zingiber officinale Rosc. \\
\hline okra & Bamia & Abelmoschus esculentus \\
\hline olive leaves & Waraq Zaytoon & Olea europaeal \\
\hline white lupine & Termos & Lupinus albus L \\
\hline
\end{tabular}

Table 2 illustrates the English, local and scientific names of herbs that were used by patients with diabetes in Jordan (adapted from Wazaify et al., 2013).

Helman (2007) suggests that health care workers are likely to underestimate the use of traditional medicine by showing negative attitudes towards it such as lack of trust and conviction towards using herbs as part of disease management. These negative attitudes of health care workers towards herbal use may hinder patients from discussing their herbal knowledge and usage with them.

A cross-sectional survey of 700 patients with chronic conditions including 260 patients with diabetes randomly selected from one hospital in Jordan aimed to explore the prevalence of herbal medicine use in Jordan (Wazaify et al., 2013). A semi-structured questionnaire method was used to collect data from participants. $7.6 \%(n=53)$ of 700 patients used herbs and $22.6 \%$ $(n=12)$ of the patients who used herbs informed their doctors about their use and the main sources of herbal information were family and friends (Wazaify et al., 2013). Health care providers could usefully increase patients' awareness of unsupervised herbal usage and encourage them to report using herbs. Herbal usage may lead to adverse effects among patients with chronic conditions in Jordan due to co-morbidities and plant-drug interactions (Wazaify et al., 2013). The data were collected based on a self-report which might be subject to recall bias. Also, the study was conducted in a teaching hospital, which might not be representative of other hospitals in Jordan where patients receive medical advice by university students of different specialities of the advantages and disadvantages of herbal use. Alkhazrajy et al. (2014) conducted a cross-sectional study with 400 health care workers in 10 primary care centres to assess their knowledge and attitudes towards herbal usage in Iraq. Most of the medical staff thought that herbs may be harmful and put the patient's health at risk. Only $50 \%$ of the participants $(n=200)$ asked the patients about herbal usage when completing their medical history. 
Different names such as folk healers, herbalists and traditional healers are given to a person who is an expert in preparing the appropriate recipes and mixtures of herbs for treating disease (Wazaify et al., 2013). In certain countries such as China, traditional practitioners have to complete official training to be qualified (Helman 2007; Payyappallimana 2010). By contrast, most of the local healers in the Arab World and developing countries including Jordan gained their experience from verbal instructions from their ancestors (Payyappallimana, 2010). Moreover, herbal knowledge is often inherited and transmitted across family members and generations without acquiring a formal education (Saad et al., 2005; Wazaify et al., 2011). For instance, $55 \%$ of 31 local healers have acquired their knowledge about plants from their older family members orally in the West Bank (Azaizeh, Fulder, Khalil, \& Said, 2003). As a result of that, herbal knowledge might be lost and distorted (Ahmad, 2016).

Safety and efficiency of herbs are important topics that are discussed in a number of different studies recently. Safety, undesirable effects, regulations, standards and preparations of herbs are examples of the main challenges that concern health care professionals and policymakers worldwide (WHO, 2014). Two Jordanian studies reported adverse effects of using plants and herbs. For instance, co-morbidities, interactions with other medications, contamination, purchasing herbs from open containers and inaccurate dosing are examples of risks associated with herbal usage in Jordan (Wazaify et al., 2011; Wazaify et al., 2013). Wazaify et al. (2013) reported that $13.2 \%$ of the 53 patients with chronic conditions in Jordan used plants and herbs in pharmacological dosage form (Wazaify et al., 2013). A scarcity of supporting clinical data for herbs and plants in comparison to conventional drugs is also considered as the main challenge of using herbs (Bone, 2013). It is recognised that many conventional medicines are derived from plants.

This section highlighted that patients tend to use herbal remedies as part of the management of their condition in Jordan. However, there is very little research conducted on herbal use among patients with diabetes or the reasons for the herbal use in Jordan. This literature review has addressed this significant shortfall; moreover, most of the patients did not inform their doctors regarding their herbal use.

\subsection{Summary}

This chapter includes information about the literature that was reviewed and accessed during the conduct of this study. This chapter has discussed the literature search strategy, critiquing 
tools and inclusion criteria used to obtain the literature which was reviewed. This literature review was organised into four themes. The first theme discusses the extent of T2DM. The second theme discusses diabetes self-management behaviours (diet, physical activity, adherence to medications, monitoring blood glucose and foot care). The third theme focuses on the knowledge, perceptions and attitudes of patients towards their condition. The fourth theme concentrates on the literature about complementary medicine and the reasons for herbal usage among patients in the previous studies.

A number of research studies were reviewed in this literature. Critiques and limitations of these studies have also been discussed. Despite the importance of social, cultural and religious factors in the Jordanian context, there is very little research conducted on the influence of these factors on the management of diabetes in Jordan. There is a shortfall in qualitative research in exploring the factors associated with the management of T2DM among patients and few qualitative studies are also available in the literature in Jordan.

This literature search and review identified that the cross-sectional survey is the most popular research design in the published articles and papers related to diabetes (27 out of 31) in Jordan and (42 out of 47) in all studies included in the literature review. This may raise concerns that cross-sectional studies represent a single point in time and may not be able to provide information about cause-and-effect relationships. Cross-sectional design or survey design is a research design in which data is collected at a single point of time by using a questionnaire or a structured interview to identify the association between two or more variables (Bryman, 2012). Also, cross-sectional studies do not address the possibility of various changes over a period of time especially adherence to long-term diabetes management behaviours such as diet, exercise, SMBG, foot care and medications which is considered as a shortfall in the literature. In the qualitative studies $(n=3)$ in this review, the small sample size was reported as a limitation which limits the generalisability of the findings of these studies. Also, there is a shortfall in research in exploring patients' family carers' perceptions of the factors that contribute to poor glycaemic levels among patients with T2DM. Although there is an important role of the family carers in patient's management of diabetes, none of the studies in this review included patients' family cares.

The data regarding adherence to diabetes management behaviours in most of the studies $(n=26)$ were based on a self-report which might be subject to recall and socially desirable answers biases. Also, another concern was the use of the same tool by asking participants to report the 
number of days of performing the diabetes management behaviours in the previous seven days. Therefore, the use of self-reported measures may be leading to recall bias and data on diabetes management behaviours were collected only once, and may not reflect long-term behaviours. In Jordan, although most of the patients with T2DM receive their diabetes treatment through primary care services, only two studies in this review were conducted in primary care settings. The sampling strategy in the most of the studies (22 out of 47 ) was convenience followed by random selection (21 out of 47 ) by recruiting patients from one clinic and not from other health care settings which limits the generalisability of the findings. Approximately a third of the studies in Jordan (11 out of 31) were conducted in the National Diabetes Centre, and the study participants were more likely to have severe diabetes and complications. Therefore, there may be an overestimation of the prevalence of diabetes complications among the study patients which may not reflect all patients with T2DM in Jordan. The National Diabetes Centre is not accessible to all people, especially who live in rural areas, which may limit the generalisation of the study findings. Also, the high prevalence of poorly controlled diabetes might explain the high prevalence of diabetes-related complications amongst the samples in the studies in this review.

The following chapter describes the methodological approach used in this study and the tools used to collect data. 


\section{CHAPTER THREE}

\section{METHODOLOGY}

\subsection{Introduction}

This chapter describes the rationale for choosing ethnography to explore the reasons behind poor glycaemic control levels in patients with Type-2 diabetes in Jordan. The aim and objectives, research paradigm, study design, study setting, sampling method, ethical considerations, recruitment process, methods of data collection, study participants, data analysis, methodological rigour and overview of theories are also discussed in detail in this chapter. Additionally, my role as a reflexive researcher throughout all phases of the research study is discussed in this chapter. The aim and objectives of the study are presented now.

\subsubsection{Aim and objectives of the study}

Aim

This study aimed to explore the reasons why glycaemic control was poor among a sample of patients with Type- 2 diabetes who used primary care services provided by a selected health care comprehensive centre in a large city in Jordan. Poor glycaemic control is defined as an HbA1c greater than or equal to 7\% (ADA, 2013).

\section{Objectives}

The following objectives were set:

1-To explore the knowledge, attitudes and behaviours of patients with poorly controlled Type2 diabetes and factors affecting adherence to their treatment plans.

2-To identify barriers to Type-2 diabetic care among patients with poor glycaemic control.

3- To explore patients', family carers' and Health care workers' (HCWs) perceptions of the factors that contribute to poor glycaemic levels among patients with Type-2 diabetes.

4-To identify patients', their family carers' and HCWs' views on factors which might facilitate improved glycaemic control levels among patients with Type-2 diabetes in Jordan. 
5-To inform the development of an educational package for patients with Type-2 diabetes based on information derived from HCWs, patients with poorly controlled Type-2 diabetes and their family carers to ensure patients' needs and concerns are addressed.

Having presented the aim and objectives of the study, the research approach that was taken is now discussed.

\subsection{Research paradigm}

This section outlines the ontological and epistemological frameworks that were followed in this research and their relationships with research design and research methods. Ontology is the philosophical study of the nature of social reality (Bryman, 2016), whereas epistemology is the theory of knowledge, relationship between researcher and the knowledge and how the knowledge can be explored and gained (Mason, 2002). In addition to ontology and epistemology, the methodology is the decisions and choices that researchers make about the research approach, participants, setting, methods of data collection, data analysis procedures and data handling, and storage in planning and conducting a study (Silverman, 2011).

The research approach consists of three interconnecting components: research paradigm, research design and research methods (Creswell, 2014). Research paradigm refers to a set of beliefs that guide the researcher's choices and decisions of research methods and interpretation of results (Bryman, 2012). The research paradigm circumscribes what is important to be examined by the researcher in a given field of inquiry (Glanz, 2002). Moreover, the research paradigm brings together beliefs and assumptions about ontology, epistemology and methodology (Denzin \&Lincoln, 2005). To explain my philosophical views, I discuss the three main theoretical paradigms: positivism, post-positivism and constructionism, and the rationale behind choosing the constructionism paradigm and subtle realism in this study.

Positivism is a study of social reality through an application of natural science methods (Bryman, 2016). Positivists believe that there is a sole, known, approachable reality, an objective reality that exists out there, independent of people's activities (Denzin \& Lincoln, 1994). This means there is a separation between researcher and the social reality in a positivism paradigm (Lincoln \& Guba, 1985). The reality in positivism is well known as "naïve realism" and can be understood through the use of appropriate methods and tools (Bryman, 2012, p. 29). In naïve realism, studying a particular social phenomenon can lead to a full understanding of 
the phenomenon's reality (Lincoln \& Guba, 1985). Within this paradigm, physical science is considered a model for social research. Research methodology in positivism tends to be experimental and manipulative in nature. The generalisation is sought within positivism and validity, and reliability is also considered as criteria to evaluate research findings (Silverman, 2011). Research in a positivism paradigm involves the development of a hypothesis to verify and to test the cause-effect relationship (linear causality) as well as between two variables, and this needs control to be exercised over the variables (Hammersley \& Atkinson, 1995).

The post-positivist paradigm has been developed in response to criticisms of positivism that there cannot be a complete truth of knowledge when studying human behaviours (Creswell, 2014). Post positivism also implies the concept of a cause-effect relationship that is found in experiments (Creswell, 2014). The ontological assumptions in post positivism propose the existence of reality out there (critical reality), but our abilities to understand this reality are imperfect and partial (Denzin \&Lincoln, 2005). Critical and naïve realists adopt the same notion which is that reality is present outside people's activities. However, critical realists believe that we are only able to gain a partial, incomplete understanding of reality, whereas naïve realists believe in the notion that we can gain a complete understanding of reality (Lincoln \& Guba, 1985).

Constructionism is an alternative research paradigm to positivism and post-positivism and attached primarily to the qualitative approach (Creswell, 2013). Researchers visit the social context where people live and act. Researchers also speak to people and interpret what has been found within the real world or in natural situations (Creswell, 2014). From this approach, knowledge, interpretations and meanings are as varied from one person to another, created through interaction with the social world and informed from historical and cultural norms and heritage (Bryman, 2012). By adopting this paradigm, the researcher tries to understand the social world that individuals live in and interpret people's meanings, views and attitudes towards their social world (Creswell, 2014). Constructionists seek to understand the meanings and interpretations beyond people's behaviours (Silverman, 2011). Unlike naïve and critical reality, the social reality in this paradigm is not viewed as a permanent external phenomenon to human beings, but in an ongoing state of accomplishments and revisions, not as a static event (Bryman, 2016). Constructionists believe that there are multiple realities with an understanding of the social world as constructed and reconstructed by human beings. Grounded theory, phenomenology and ethnography are examples of research approaches that are underpinned by constructionism (Glanz, 2002). 
Hammersley (1992) rejects naïve realism and proposes subtle realism as a way forward especially for ethnography. Hammersley (1992) postulates that subtle realism lies midway between naïve realism and anti-realism (multiple degrees of reality). Hammersley shares the concept of external reality with positivists. However, Hammersley (1992) has argued that there is no separation between the researcher and social reality; both the researcher and the participants construct and engage in the formation of this reality. Additionally, there is no direct complete, reliable access to social reality which means we can never be certain about the truth of the studied social phenomenon or people (Bryman, 2012).

Hammersley (1992) challenged three elements of realism: firstly, that knowledge is defined “as beliefs whose validity is known with certainty" (pg. 50). In this case, researchers cannot have any knowledge because they cannot be absolutely sure about the truth of our claims. Hammersley (1992) offered an alternative definition of knowledge that is " beliefs about whose validity we are reasonably confident', (pg. 50). That is, no knowledge is certain, and claims can only be judged or based on credibility, plausibility and assumptions which the researcher holds about the world currently as being beyond reasonable doubt. Secondly, researchers' claims do not alter individuals' realities which may or may not represent their realities accurately. In other words, the social reality is not independent of us and we engage in the construction of it. Thirdly, that social research aims to represent rather than reproduce the reality, and the same phenomenon can be addressed and presented from multiple perceptions (Hammersley, 1992).

\subsubsection{Ontological and epistemological frameworks of this study}

Neither the ontological or epistemological stance of positivism and post-positivism were consistent with the aim of this research because they both focus on objectivity and control. The aim and objectives of this study could not be achieved through manipulative and experimental methods derived from positivism and post-positivism paradigms. Therefore, the constructionism paradigm and subtle realism were relevant and deemed most appropriate for addressing the aim of this study.

To date, there are no identified, published studies that have sought to explore the subjective meaning behind patients' health behaviours and the influence of socio-cultural norms on the management of T2DM and the high prevalence of poorly controlled Type-2 diabetes in Jordan. Therefore, the constructionism paradigm and incorporating subtle realism supported my 
research approach (qualitative) to generate rich and holistic information to gain an in-depth understanding of participants' meanings and interpretations influencing their behaviours.

The paradigm of constructionism and subtle realism also supported my research methods aimed at identifying and understanding individuals' meanings, views, experiences and the influence of their social and cultural world in which they live on their management of Type-2 diabetes. My epistemological stance and research methods such as participant observation, focus groups and interviews were based on interaction and communication with study participants and using open-ended questions to identify and share their concerns and ideas. This indicates that I was not embracing an objective/subjective stance with participants as in positivism and postpositivism whereby the researcher and participants are independent and separated (Lincoln \& Guba, 1985) and the role of the researcher is to remain objective and neutral. In contrast, I acknowledge that my beliefs, attitudes and knowledge regarding T2DM and its management have influenced the choice of topic to be studied, the aim and objectives which have been set, and the methodology and methods to be used. In order for the reader to judge how my beliefs and values have influenced the entire research study, I adopted a reflexive approach. By doing this, the reader can judge whether the findings of this study are credible and plausible.

My philosophical views which are consistent with the constructionism paradigm and subtle realism, besides the aim of the study, influenced choosing qualitative research as an appropriate approach to conduct this research study. The rationale for choosing a qualitative approach and the ethnographic design is discussed in the following section.

\subsection{Qualitative research approach}

Bryman (2012, p. 714) defines qualitative research as an approach that, ' usually emphasises words rather than quantification in the collection and analysis of data.' Qualitative research focuses on collecting data in natural settings rather than artificial settings such as laboratories and experiments (Denzin \& Lincoln, 1994). Naturalism is achieved when the researcher tries to minimise using artificial methods of data collection (Bryman, 2012). Moreover, the researcher observes the participants' behaviours and communicates with them in their social setting (Creswell, 2014). This study sought to uncover rich information and allow patients and their family carers to share their concerns and needs about living with Type-2 diabetes in Jordan. 
Qualitative methods have been used to access areas in social life that are not available to quantitative methods (Pope, Ziebland, \& Mays, 2000). Creswell (2007), states that one of the significant sources of data is when people express themselves, and their needs and obstacles that affect their lives openly and without restrictions. Qualitative research enables HCWs and policymakers to focus more on the patient's health care needs which can be used to inform the health policy-making process (Bowling, 2014). In qualitative research, the developing theory is an inductive process which means the theory emerges and is generated from the data (Morse \& Field, 1996).

Most of the existing studies related to diabetes in Jordan used quantitative methods primarily and always used a questionnaire as the data collection method. A cross-sectional design has dominated most of the published Jordanian literature. Cross-sectional design or survey design is a research design in which data is collected at a single point of time by using a questionnaire or a structured interview to identify the association between two or more variables (Bryman, 2012). Cheaper, easier and quicker to conduct, the absence of interviewer's bias and limited influence on the participants' responses are the main advantages of the cross-sectional survey (Bryman, 2012). However, these approaches and methods would have provided me with a superficial understanding of people's behaviours and perceptions that would not be sufficient because my study's aim was to generate rich and holistic information about the factors contributing to poorly controlled Type- 2 diabetes from the patients' perceptions, their family carers' and HCWs' perceptions. Additionally, in order to develop a questionnaire, I would have needed to know great deal about the socio-cultural concerns of people with Type- 2 diabetes and this to date has not been sufficiently explored in Jordan.

To date, there are no identified, published studies that have sought to explore the meaning behind patients' health behaviours and the influence of socio-cultural norms on the management of Type- 2 diabetes and the high prevalence of poorly controlled diabetes in Jordan. Morse and Field (1996) suggested that qualitative research should be used when there is a lack of previous studies about a particular phenomenon. A qualitative approach was the most appropriate choice for this study because of the research aim and objectives were concerned with gaining an in-depth understanding of factors associated with poorly controlled Type-2 diabetes in Jordan.

The main qualitative research designs are grounded theory, case study, phenomenological research and ethnography (Morse \& Field, 1996). Although all qualitative research is 
concerned with developing and revising understanding, rather than purely verifying earlier conclusions of theories, different qualitative methodologies have diverse foci, and many accomplish their aims in different ways. Grounded theory and case study designs were not considered for this study for the following reasons.

Grounded theory is a qualitative method where the researcher analyses the data to generate a theory that is grounded and derived from the research data (Bryman, 2012). The researcher should eliminate any preconceptions or previous thoughts or prejudice before approaching the study field and allow the collected data to guide and generate the developed theory (Morse \& Field, 1996). However, the researchers may experience difficulties in eliminating their awareness of existing theories and literature until the late stages of the study (Bryman, 2012). I had my preconceived ideas about the social world of patients with Type-2 diabetes which could not be avoided and excluded. My study also aimed to add valuable contributions to the existing literature and fill a shortfall in the literature regarding poorly controlled Type-2 diabetes in Jordan. This means I conducted a detailed literature review to identify what was already known about Type-2 diabetes in Jordan. Additionally, the goal of grounded theory design is to generate inductive theory (Silverman, 2011) which was not one of the objectives of this study.

The case study is another qualitative research design which focuses on one to three cases for extensive and detailed exploration (Bryman, 2012). Participant observation and unstructured interviews are considered the most appropriate methods to collect detailed data in the case study design (Bryman, 2012). Though aspects of the case study design were evident in my study as I looked at one comprehensive health care centre which could have been considered as one case. However, the focus of my study was not the centre per se but the perceptions of the patients, their family carers and HCWs regarding the factors which facilitated and the barriers which impacted on poor glycaemic control. Also, focusing only on a single case study was not the objective of this study. My study aimed to collect data from multiple perspectives and a wide range of participants.

Two qualitative designs were considered as potentially being appropriate to address the study's aim and objectives namely phenomenology and ethnography. Phenomenological research is a research method and philosophy designed to explore and understand people's every day lived experiences about the particular phenomenon (Creswell, 2014). The goal of the phenomenological research is to provide a detailed description of people's experiences of the 
studied phenomenon with the aim of exploring the essence of meaning (Morse \& Field, 1996). As this design focuses on gaining a deeper understanding of the meaning of a particular human experience, it was not appropriate because my focus was on reasons rather than sense-making. This study also aimed to collect multidimensional data from different sources to gain a more fully comprehensive picture of the factors which influence glycaemic control of Type-2 diabetes within the context of Jordan which may only be achieved by prolonged engagement in the social setting (Mason, 2002). Additionally, my focus was not solely on the lived experiences, although that is an aspect I was interested in exploring. I was interested in understanding the wider social and cultural factors which may influence the management of Type-2 diabetes, and for these reasons, I rejected this approach. The following section includes a description of ethnography and the rationale for choosing it as the methodological design for this study.

\subsubsection{Ethnographic design}

An ethnographic design was used in this study to explore the factors associated with poorly controlled Type-2 diabetes in Jordan. The ethnographic design enabled me to understand shared and common experiences among patients with poor glycaemic control levels in their social, cultural and economic contexts.

The roots of ethnography are related to the early 1900s, to Malinowski and Radcliffe-Brown, who spent long periods of time in rural societies to document their social interactions, norms and belief systems (Reeves, Kuper, \& Hodges, 2008). Ethnography came from cultural anthropology with a focus on understanding shared common cultural practices among a group of people (Morse \& Field, 1996). The ethnographic design was later adopted by a group of sociologists at the Chicago School of Sociology and applied to study a variety of cultural groups (Creswell, 2007). The concept of ethnography consists of two words: ethno means 'folk' or people and graph refers to "writing"' (Silverman, 2011, p. 114). Ethnography is a qualitative design in which the researcher spends an extended period in studying shared cultural, social and behavioural patterns of a social group of people in their natural setting (Creswell, 2014). The ethnographer uses multiple methods of data collection mainly participant observation and semi-structured or unstructured interviews (Creswell, 2014). The term ethnography can be applied to a method of research and the written product of the research (Pellatt, 2003). The ethnographers describe, unfold and transfer "what is there" in the field to an interpretive 
written product that displays life experiences of people and their relationships/interactions with the society (Muecke, 1994, p. 191).

Ethnography focuses on the entire cultural group and the relationships between the people and their natural environment. In ethnography, the researcher is required to spend time immersed in the participants' social setting to observe their behaviours, beliefs and language (Creswell, 2007). So the focus is not only on the observed or visible behaviours but also attitudes, beliefs and communication, either verbal or non-verbal. Ethnographers usually choose only one setting or a few settings to conduct their detailed research studies due to the cost of travel to remote areas and limited resources available (Hammersley \& Atkinson, 1995). Additionally, choosing one setting enables the ethnographer to generate rich information about the participants and to spend more time in the selected setting (Hammersley \& Atkinson, 1995). Ethnographic studies aim to generate a rich and in-depth understanding of the shared social group's behaviours, beliefs' and perceptions' (Reeves et al., 2008). One of ethnography's strengths is that the researcher spends time exploring social behaviours through participant observation incorporated by interviewing the cultural group to gain an in-depth understanding of their behaviours (Hammersley \& Atkinson, 2007).

Culture is an important aspect of the patient's thoughts about health and illness (Helman, 2007) and ethnography is informed by the concept of culture (Morse \& Field, 1996). The concept of culture is known as a system of shared ideas, concepts, rules and meanings that are expressed in the ways that people live (Helman, 2007). Ethnography enabled me to understand the wider social and cultural factors which may influence the management of Type-2 diabetes in Jordan. Ethnography has become increasingly used in studying in-depth health care issues in their natural settings such as the delivery of health care, health beliefs and health and illness behaviours (Savage, 2000). Ethnography is being used to explore the impact of patients' cultural practices on their treatment plan, and the findings may aid HCWs in understanding patient's culture (Savage, 2000).

Focused ethnography is a common ethnographic design that is used widely in the nursing field. Nurses prefer to use focused ethnography rather than classical ethnography to improve specific nursing knowledge and particular practice (Muecke, 1994). Focused ethnography is a branch of ethnography characterised by the short and focused duration of participant observation that differs from the classical picture of ethnography with long-term fieldwork (Knoblauch, 2005). Data are collected in focused ethnography through episodes of participant observation which 
includes interviews (Muecke, 1994). Focused ethnography was chosen in my study and the main reasons for using focused ethnography were the constraints of time and resources. I could not have spent years in the study setting as I had to complete my $\mathrm{PhD}$ within a defined time frame. Also, this study was focused in the primary care setting as that is the setting where people access health care and their treatment plans are negotiated with HCWs. The study setting where I conducted my ethnographic study is discussed now.

\subsection{Study setting}

This study was conducted in a large comprehensive health care centre that provided primary care services for people who live in a large city in Jordan (section 1.5). Ethnographers usually choose one or a small number of settings to conduct their ethnographic studies (Hammersley \& Atkinson, 1995). The ethnographer has to take into account choosing the appropriate fieldwork site and the right period for conducting participant observation to provide him/her with good quality data (Silverman, 2011). In the selected centre, scheduled family medicine clinics were operating from Saturday through Friday between 0800 and 1400 which enabled me to generate good and rich observations regarding patients with T2DM. I also lived close to the centre which helped with reducing travel costs as I was self-funding my $\mathrm{PhD}$.

This study sought information from different groups of participants by using multiple methods of data collection which made the sampling method a complex process. The sampling method is discussed next.

\subsection{Sampling method}

The data collection process in qualitative research has to be efficient and effective due to multiple reasons such as the small sample size, generating too much data, time and cost constraints (Morse \& Field, 1996). The principle of random selection is inappropriate in qualitative research as randomly selected participants might provide me with superficial or no information about my research topic (Morse \& Field, 1996). In qualitative research, cases are often selected based on accessibility to them (Silverman, 2011). My study aim was to generate rich and holistic information regarding T2DM. Additionally, this study aimed to explore the reasons for poor glycaemic control levels particularly among patients with T2DM aged over 40 years, because of the prevalence rate of T2DM in Jordan rises after the age of 40 years in both sexes (Ajlouni et al., 2008). Additionally, to seek the views of patients' family carers and 
HCWs who provide direct care to patients with T2DM. For these reasons, therefore, purposive sampling was the most appropriate sampling method for this study. Purposive sampling is a type of non-probability sampling when the researcher selects study participants with a purpose, so the selected participants have the knowledge and experience to address the research aim and objectives (Bryman, 2012).

As qualitative research aims to generate rich, in-depth data, sample sizes tend to be smaller than in quantitative studies (Creswell, 2014). However, it is difficult to suggest a sufficient number of participants that must be included in qualitative research (Bryman, 2012). Bernard (2000) states that most ethnographic studies include between 30-60 participants. Guest, Bunce, and Johnson (2006) have argued that qualitative research should include at least 15 participants. The sample size for participants in the focus groups and interviews will be discussed later in this section.

Morse and Field (1996) introduced two main principles to enable researchers to select study participants in qualitative research: appropriateness and adequacy. Qualitative research findings can be superficial and of poor quality if the sampling criteria of appropriateness and adequacy have not been met (Morse \& Field, 1996). Appropriateness was addressed in this study through the selection of the most knowledgeable participants regarding this research topic, namely patients with poorly controlled T2DM, HCWs who provide formal care and family carers who provide informal care to patients with diabetes. These three groups have the knowledge to talk about their experiences and views regarding T2DM and the factors which may hinder and help in maintaining blood glucose levels within normal limits.

In addition to the appropriateness, the principle of adequacy was applied. The indicative total sample size for patients was 25 participants, for HCWs was 12 participants, and for patients' family carers, it was 10 participants with more if needed to reach data saturation. This sample size was considered adequate for addressing the research aim and objectives, although it was acknowledged that my intention was to develop a rich and full understanding of the phenomenon of poorly controlled Type-2 diabetes in Jordan and that my sample may need to be expanded in light of emerging issues arising from data analysis or until I reached data saturation. According to Bryman (2012), data saturation is the endpoint in the data collection process when no new material or comments are generated. The principle of purposive sampling was applied in this study relating to participants, observations, time, place and documents 
found in the study field (Bryman, 2012). First of all, sampling during participant observation will be discussed.

\subsubsection{Sampling for participant observation}

The initial plan was to conduct participant observation in the waiting area in the selected comprehensive health care centre. However, it became evident that patients were only sitting in the waiting area speaking to each other and waiting to be called to enter the doctor's consultation room. Therefore, I did not conduct participant observation in the waiting area as I would not have been able to generate rich and adequate data.

I adopted Hammersley and Atkinson (1995, p. 45) principle of "sampling within the case." I had my predetermined agenda that guided participant observation. This agenda is mentioned later in the methods of data collection section. The sampling of time is another feature of participant observation as to which observations should be conducted on different occasions and different periods of time (Bryman, 2012). I aimed to conduct my observation episodes at different times of the day and on different days in the family medicine clinics. I was observing participants in different areas such as family medicine clinics, the pharmacy and the nurse's room focusing on the length of time that patients spent in the centre, length of time that patients spent with doctors or nurses, the discussed topics, patient-doctor communication, provision of health education, whether patients attended alone or with others and accessibility to the centre. I also participated in the diabetes education programme provided in another centre by taking notes about the information given and observing the style of the information given. In total, I conducted 80 hours over a four month period which included all the days of the week the family medicine clinics were open.

With participants' permission, all data related to patients' HbAlc levels were obtained from patients' files by the HCWs. That enabled me to recognise patients with poorly controlled T2DM and facilitated observations of patients with poor glycaemic control levels. During participant observation, I conducted informal conversations with the HCWs, patients with poorly controlled Type- 2 diabetes and their family carers. In addition to sampling during participant observation, patients for focus groups were sampled based on inclusion and exclusion criteria. The sampling process for patients is discussed now. 


\subsubsection{Sampling for patients}

The aim and objectives of this study were concerned with patients with Type 2 diabetes who had poor glycaemic control. Inclusion criteria included patients diagnosed with Type 2 diabetes who had a HbA1c greater than or equal to $7 \%$. According to the American diabetes association (2013), a HbA1c reading greater than or equal to $7 \%$ is considered to be poorly controlled Type- 2 diabetes.

Inclusion criteria included patients with Type- 2 diabetes who lived in the selected city and attended the selected health care centre to receive diabetic care. It has been reported that patients with Type-2 diabetes need one year since diagnosis to understand and monitor their disease (Al-Khawaldeh et al., 2012). In order to avoid an unnecessary additional burden for patients who had been newly diagnosed, the inclusion criteria included patients who had been diagnosed with Type-2 diabetes for more than one year.

Ajlouni et al. (2008) reported that the prevalence rate of Type 2 diabetes in Jordan rises after the age of 40 years in both sexes. Globally, 374 million out of a total of 425 million people with diabetes (all types) are aged more than 40 years old (IDF, 2017). This study included patients with Type-2 diabetes who were older than 40 years. Additionally, the study participants needed to be able to communicate in Arabic, to understand and be able to give informed consent and be willing and able to participate in a focus group and/or an interview. The exclusion criteria are listed below including people who had severe speech or hearing difficulties and who had a serious cognitive impairment.

\section{Patients' exclusion criteria}

1- Patients with Type-2 diabetes who had severe speech or hearing difficulties.

2- Patients with Type-2 diabetes aged less than 40 years old.

3- Patients with Type- 1 diabetes or gestational diabetes.

4- Patients with Type- 2 diabetes with good glycaemic control levels HbA1c less than 7\%.

5- Patients with a serious cognitive impairment who were unable to give informed consent.

6- Patients who received their diabetic care from hospitals or another diabetic care centre.

7- Patients with Type-2 diabetes who have been diagnosed with diabetes for less than one year. 
Besides patients, family carers and HCWs were crucial sources of information regarding the management of Type-2 diabetes among patients. Sampling for HCWs and family carers will be discussed next.

\subsubsection{Sampling for HCWs}

In order to gain different perspectives, I interviewed HCWs from different specialities including nurses, doctors, pharmacists, reception staff, phlebotomists, dentists and dietitians. My potential participants were HCWs who provided formal care to patients with Type-2 diabetes and who had worked for more than six months in the selected centre. I selected participants with more than six months of experience in providing care for patients so that they had knowledge and experience of providing care to this patient group and because all patients with Type-2 diabetes should have a HbA1c test done every 3-6 months (ADA, 2013). More than six months of experience was considered appropriate and adequate, which enabled me to select HCWs who provided care for all patients with Type-2 diabetes who had this length of experience. The average number of HCWs in the selected health care centre was 55 people. The total sample size for HCWs was 10 participants. To gain different perspectives and views, I conducted interviews with two nurses, one doctor, one pharmacist, one reception staff, one diabetic nurse, one dentist, one lab technician and one dietitian. I also went to the Ministry of Health to interview the supervisor of Health Promotion programmes in the Ministry.

\subsubsection{Sampling for Family carers}

In addition to HCWs, I also interviewed patients' family carers. Patients spend most of their time with family carers, therefore, including family carers added different perspectives and views from the community that added another valuable source of information to address my research aim. My potential participants were people who provided informal care to patients with Type- 2 diabetes in the patients' home. Family carers were identified by patients with Type2 diabetes and included relatives, family members and significant others such as friends. The total sample size for patients' family carers was 6 participants. I interviewed patients' family carers with different caring relationships such as one husband, two sons and three wives.

The sampling process was not only limited to the sampling of people, but documents were also sampled purposively based on the aim of the study (Bryman, 2012). The sampling of documents is discussed now. 


\subsubsection{Sampling of documents}

Documentary data is the main method of data collection in classical sociology (Scott, 1990). Ethnographers should evaluate the data that is extracted and derived from evidentiary documents as they do with the collected data from other methods of data collection such as interviews and participant observation (Davies, 2008). Documentary data is also part of people's social setting and should be taken into account during conducting ethnographic research to gain more understanding of their culture (Hammersley \& Atkinson, 1995).

The documentary review aimed to gain an understanding of the study setting and also to identify whether the information in written documents was considered as a factor which hindered or facilitated in maintaining patients' blood glucose levels within normal limits.

The educational documents accessible for review included the current diabetes education programme, attendance registers and diabetes educational leaflets that were provided in the centre during the time of data collection. These written documents were reviewed and investigated based on Scott's evaluation criteria (1990). Scott (1990) proposed four criteria for judging the quality of documentary evidence; these are authenticity, credibility, representativeness and meaning.

Authenticity addresses the question, "is the evidence genuine and of unquestionable origin?' (Scott, 1990, p. 6). Were the documents that had been found in the centre used to educate patients with diabetes? Whether the documents available in the centre were actually evidence-based and from unquestionable sources or not? If yes, what was the original source and was there any editing or copying or translating from the original source? In addition to authenticity, credibility is another criterion to evaluate the evidence which refers to the accuracy and honesty of documents (Davies, 2008). I was interested in who developed the educational materials in the centre and why did he/she decide to select these types of information and exclude others? Additionally, were the materials free from errors and distortion?

The third criterion, which is representativeness means "'is the evidence typical of its kind, and, if not, is the extent of its untypicality known?" (Scott, 1990, p. 6). I was interested in the number and type of relevant documents that had been produced in the centre and whether current available documents were typical of those provided by the centre. The final criterion is 
meaning which is concerned with the clarity and comprehensibility of the evidence (Scott, 1990). The meaning of educational materials was evaluated by asking patients during the focus groups and participant observation whether they were able to read and understand it. Did the materials contain any technical terms or unfamiliar language? Were the educational materials appropriate for people in Jordan? Did the educational materials take into account the cultural and social norms in Jordan? In developing the educational materials, were patients involved in the process or not?

After providing the reader with information about the sampling strategy used, I discuss the recruitment process now.

\subsection{Recruitment process}

\subsubsection{Initial approach}

I had a meeting with the director of the comprehensive health care centre. During that meeting, I explained to him the aim and objectives of my study. I also conducted a presentation to HCWs in the selected health care centre. In the presentation, I explained the aim and objectives of the study and the methods of data collection. I also explained the inclusion and exclusion criteria for selecting the patients for the focus groups and all the HCWs were given the opportunity to ask questions, and all questions were answered. Then I gave information packs which included the invitation letter (Appendix 4), reply slip (Appendix 5) and participant information sheet for the patients for the focus group to HCWs, and they were asked to distribute the information packs to potential participants who met the inclusion criteria. I also invited the HCWs to participate in this study and gave them the participant information sheets. For participant observation, I, with permission, conducted participant observation in different areas in the centre such as the consultant's room, pharmacy, waiting area, nursing room and the conference room. The director also gave me the approval to attend any formal educational session about diabetes in the centre.

\subsubsection{Recruitment of samples}

For focus group participants, I provided patient information packs to HCWs who worked in the selected health care centre. My contact details (telephone and email) were written in the participant information sheet (Appendix 6). Patients who were interested in participating had two choices; either they preferred to contact me directly or if they preferred, I contact them by telephone or email. Participants were also asked to complete a short sociodemographic 
questionnaire when they attended the focus group session. At the first contact with participants, I gave more information about the date, venue and time of the focus group session. I informed the participants that they would receive a glucometer which was equivalent to 12 Jordanian Dinar (approximately 12 pounds sterling) as a token of appreciation for their time and assisting them with the management of their diabetes, as self-monitoring blood glucose levels is an important element in diabetes management behaviours and many patients did not have it. For patients who agreed to take part in a focus group, I contacted them two days before the scheduled focus group session to confirm attendance and remind them of the time and venue of the focus group. I also reminded them they could withdraw at any time and without giving a reason. During my time conducting participant observation, I also invited patients to take part in the research. Two patients who took part in the focus groups were recruited by me during participant observation. The rest were recruited by the HCWs.

I recruited HCWs who provided formal, direct care to patients with Type-2 diabetes. I gave the participant information sheets (Appendix 7) to HCWs during the planned presentation. For HCWs who did not attend the presentation, I handed out the participant information sheets when I had the opportunity to meet them. Nine HCWs who responded positively were given two weeks to the date of the interview to think about participating. I made it clear to the study participants that they had the right to withdraw at any time without any negative consequences.

For patients' family carers, I provided patient information sheets (Appendix 8) to HCWs who worked in the selected health care centre. Additionally, I asked the focus group participants if their family carers would be interested in taking part in this study. Focus group participants who responded positively, were given the participant information sheets to hand out to their family carers. Four family carers who took part in the study were recruited through focus group participants and two family carers were recruited by the HCWs. Patients' family carers were given the opportunity to choose the interview time and venue. Patients' family carers who agreed to participate were invited to interview. All the questions were answered before written consent (Appendix 9) was gained and the interview took place. The interview lasted approximately 30 minutes and all chose that the interview was conducted in a private room in the selected health care centre. In the following section, the methods of data collection are discussed, and the rationale for selecting them is also provided. 


\subsection{Methods of data collection}

\subsubsection{Introduction}

Data in this study were collected through multiple methods of data collection including participant observation, focus groups and semi-structured interviews, documentary review and informal conversations. A reflexive journal was kept and used as an additional source of information. Firstly, participant observation is discussed.

\subsubsection{Participant observation}

Participant observation is a method of data collection that enables the researcher to immerse himself/herself in the participants' research setting for a prolonged period. The researcher watches participants, records events and is involved in conversations with the study participants (Bryman, 2012). Participant observation was conducted in the different areas in the selected health care centre. Permission to conduct participant observation was secured from the director of the selected health care centre before the observations took place.

Information gained from participant observation was written up in field notes. In addition to participant observation, field notes might be used to record data from each method of data collection in ethnography (Davies, 2008). Field notes also encompassed any non-verbal responses in the focus group or interview discussion, a floor plan of the setting and a physical map of the selected health centre.

There are two types of field notes, descriptive and reflexive. Descriptive information is the objective part of the field notes and includes a description of the physical setting, people's behaviours and conversations between people. In addition to descriptive information, reflective information is the subjective part of field notes and focuses on feelings, impressions, concerns, and ideas of the observer (Bogdan \& Biklen, 2003).

I organised and maintained separate sets of field notes including written notes during each observation episode, immediate reflexive notes following each completed field session or focus group or interview, a full record of ideas and problems which emerged from each fieldwork session, and preliminary record for analysis and interpretation of field notes. An ethnographer should also keep the recorded subjective and objective field notes separately (Morse \& Field, 1996). Throughout conducting the study, I kept the written field notes as a key source of information enabling me to understand the reasons behind poorly controlled Type- 2 diabetes in Jordan. 
Throughout the data collection, I wore an identification badge saying, 'I am a $\mathrm{PhD}$ research student'. This identification badge was worn in a readable and visible manner and let patients know the purpose of my presence in the areas where I conducted participant observation. That enabled me to be introduced to and identified by patients in the selected areas as a research student who was conducting a research study. Furthermore, the identification badge aided recruitment of participants for the focus groups.

The observations focused on the length of time that people with poorly controlled Type- 2 diabetes spent in the centre, the length of time that patients spent with doctors or nurses, the discussed topics, patient-doctor communication, whether patients attended alone or with others, accessibility to the centre and whether patients read information material provided by the centre on notice boards and leaflet racks.

The health education programmes had stopped in the selected centre for more than six months before I conducted my study. Therefore, I participated in the nationally approved diabetes education programme provided in another centre by taking notes about the information given and observing the style of information giving. This health education programme was the same programme delivered across the country.

During the observations, I conducted informal conversations with the HCWs, patients with poorly controlled Type-2 diabetes and their family carers. During these discussions, I reminded those whom I spoke with that I was a researcher and involved in conducting a research study. The researcher can perform multiple roles in the field of the study during participant observation. These roles and examples of adopting these roles in my study are discussed in the following section.

\subsubsection{Roles of the researcher during participant observations}

Participant observation is commonly used to obtain data in ethnography (Bryman, 2012). During participant observation, there are four roles of field worker based on interactions with participants: complete observer, observer-as-participant, participant-as-observer and complete participant as illustrated in Figure 5 with different degrees of observations (Gold, 1958).

As a complete observer, there is no risk of going native and it is also suitable for the exploration of the field to prepare for more intensive study. However, there are no social interactions with participants which can result in misunderstanding issues (Gold, 1958). I did not aim to be a complete observer as I wanted to conduct informal conversations with participants to aid 
understanding of the meaning behind the behaviours observed and to recruit participants for focus groups. In contrast, there is a complete participant role whereby the field worker fully participates with participants which may be incorporated by covert observations (Gold, 1958). Data collection is also constricted by social practices and norms which may limit the ability of the field worker to optimise the data collection process (Hammersley \& Atkinson, 1995). Additionally, it is difficult for the researcher to act in dual roles as being a member of the studied group and a researcher who observes them objectively at the same time (Morse \& Field, 1996). Besides the above reasons, covert observation raises ethical issues and impedes data collection, so this role was considered inappropriate.

Between complete observer and complete participant, there are observer-as-participant and participant-as-observer (Hammersley \& Atkinson, 1995). As an observer-as-participant, there is superficial contact with participants, more formal observations and an opportunity for misunderstanding by both researcher and participants (Gold, 1958). As a participant-asobserver, there is mutual awareness between researcher and participants which means I was identified and introduced myself as a registered nurse and a researcher who was conducting the research study. However, there is the possibility of 'going native' (Gold, 1958, p.221) and the potential risk of developing an intimate form of relationship with participants (Gold, 1958). Therefore, the skills of the researcher are crucial in conducting fieldwork in two aspects; the ability of the researcher to create a good rapport with participants so they feel comfortable to engage in the study, and being aware not to immerse himself/herself deeply as an insider so he or she might lose his/her outsider perspective (Giddens \& Griffiths, 2006).

Going native refers to the situation when an ethnographer loses his/her identity as a researcher and adopts participants' views and perspectives (Bryman, 2012). The risk of going native may arise from an over rapport with the people being studied and from seeing the social world through their eyes (Bryman, 2012). The researcher should keep more or less a marginal position in his/her contact with participants (Hammersley \& Atkinson, 1995). During participant observation, the researcher should be able to access participants' perspectives but at the same time avoid over rapport with them (Hammersley \& Atkinson, 1995).

My role in the health care centre was both as a participant-as-observer and observer-asparticipant (Gold, 1958). Adjusting fieldwork roles is important during observations to achieve the objectives of the research (Gold, 1958). Adopting different fieldwork roles enables the researcher to gain access to different sorts of data and to make sense of types of bias in each 
adopted field role (Hammersley \& Atkinson, 1995). I moved between roles at different times to maximise gaining information. For instance, being a participant-as-observer, I conducted informal conversations with the HCWs, patients with poorly controlled Type-2 diabetes and their family carers. I also participated in the diabetes education programme provided in the centre by taking notes about the information given and observing the style of information giving. Being an observer-as-participant, I conducted observations focusing on the length of time that people with poorly controlled Type-2 diabetes spent in the centre, whether patients attended alone or with others, accessibility to the centre and patient-doctor communication.

Observer-as- participant

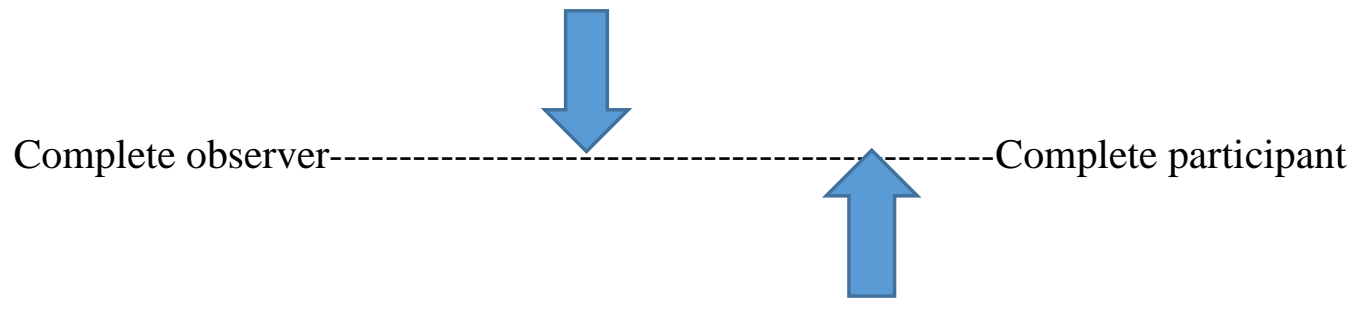

Participant-as- observer

Figure 5 illustrates roles of researcher during participant observations (Gold, 1958).

The focus group and interview guides were informed by the emergent findings gained from participant observation such as discontinuity of care and herbal usage. During participant observation it became evident that patients used herbs and plants for the management of diabetes. During observations, patients told me informally about the use of herbs. Exploration of herbal use among patients offered useful insights into the role of the socio-cultural factors on the self-management of diabetes, so an extra question was framed and added to the focus group guide: Do you use herbs for management of diabetes? If yes, why do you use herbs?

During participant observation it also became evident that discontinuity of care affected the patient-doctor relationship and patients' adherence to their management plan. It was also clear that discontinuity of care was time consuming for the patients and also for the doctors. I explored this during focus groups by adding an extra question to the focus group guide: How do you feel seeing different doctors every time you visit the centre?

Additionally, through my observations at the clinic I noted that there were long waits for patients in the different departments which was inconvenient for them. It was evidenced from participant observation and from patients' interviews that the long waiting times added stress 
and concern for patients and their family carers at an already distressing time. It was noted during participant observation that the nurses and doctors did not often engage in conversations with patients even when there seemed to be time, especially when the centre was quiet. These issues were explored further by asking patients and their family carers during focus groups and interviews: What are the challenges that patients face during their visit to the centre?

In addition to 80 hours of participant observation, eight focus group were also conducted which are now discussed.

\subsubsection{Focus groups}

According to Bryman (2012), a focus group is a qualitative technique that involves interviewing a small group of people sharing a particular interest and discussing a study topic with the guidance of a moderator or facilitator. Seeking rich data is considered as one of the main reasons behind conducting focus group discussion (Carey, 1994).

Focus group discussion encourages participants to express their beliefs, perceptions, concerns, feelings and attitudes behind their behaviours (Carey, 1994). It also facilitates social interaction between participants themselves and with the facilitator (Morse \& Field, 1996). The focus group discussion provides an excellent opportunity for participants to speak and listen to each other, ask questions, exchange knowledge and help each other. The facilitator can explore and probe participants' knowledge, interaction and experiences (Kitzinger, 1994). So focus group discussion was chosen as a method of data collection in this study for the previous reasons stated.

The recommended focus group size is 6 to 10 people (Morgan \& Krueger, 1998). Small group size stimulates discussion and encourages participants to share their ideas and enables the facilitator to explore any tensions, disagreement or conflicts between group participants. A minimum number of four focus groups were planned with more if needed to reach data saturation. Eventually, I conducted eight focus groups with 38 patients, and I aimed for 4 to 6 patients in each group. Focus groups included female and male patients together as this is culturally acceptable in Jordan. Thereby, I used a female dietitian as an assistant to aid me in conducting focus groups as the presence of a female assistant made female participants more comfortable during the focus group discussion.

Focus groups were conducted by me, aided by an assistant with a small group of patients (4-6) with poorly controlled Type- 2 diabetes. I acted as the facilitator who guided the focus group 
discussion and the other person acted as a focus group assistant who took notes during the discussion. The assistant did not participate in the focus group discussion, and she noted any non-verbal responses related to the questions posed. Additionally, the assistant ensured the audio-recorder was working effectively during the focus group session (Krueger \& Casey, 2002). Patients were asked to talk about their life experiences and perceptions in relation to T2DM, the barriers and facilitators to glycaemic control and their sources of information.

These topics had been derived from the aim of the study, reviewing the literature, expert recommendations and were informed by the participant observation. To test the clarity and relevance of the focus group topic guide (Appendix 10), it was piloted with a group $(n=3)$ of Jordanian patients with Type-2 diabetes who received care from the selected health centre. The focus group topic guide was translated from English to Arabic by me and was translated back into English by a bilingual translator to verify the accuracy of my translation. Before the focus group started, the focus group assistant and I arranged the room, prepared glucometers, the audio-recorder and all papers related to the focus group discussion. The audio recorder was used with permission from all focus group patients.

Ground rules were established to seek to preserve the confidentiality of the discussion between patients and all patients were asked to follow these rules and that they agreed to maintain the confidentiality of the discussion. Patients were given the opportunity to ask questions related to the information sheet, and all questions were answered before the focus group session took place. Then, all patients were asked to sign the consent form (Appendix 9) and complete an anonymous sociodemographic data questionnaire (Appendix 13).

A sociodemographic data questionnaire had been developed by me based on the Jordanian literature, the objectives of the study and expert recommendations. It was given to the patients at the beginning of the focus group session to collect data on the following: age, gender, highest level of education, duration of Type-2 diabetes, type of treatment, family history of diabetes, smoking, body mass index (BMI), weight, marital status, monthly income and attendance at a formal diabetes education programme in the last year.

I started the focus group session by explaining the purpose of this study, the focus group process and sought permission for the use of the audio recorder. I started the focus group discussions by following the focus group guide, and the FG assistant started taking notes and comments. After the topics had been covered, I asked all the patients if they wanted to add any additional information. If the patients did not have any more information to add, the focus 
group session ended. A debriefing session was conducted after each focus group, so patients could express their reactions and understandings of the discussions in the focus group and ensure that no one left feeling upset, and this aided in maintaining confidentiality. During the debriefing sessions, I used a whiteboard to write up the key issues discussed during the focus group including any misconceptions which were then addressed to ensure that all participants were aware of accurate information regarding the management of Type- 2 diabetes. As a qualified nurse and researcher, incorrect information and misconceptions were addressed during debriefing sessions after each focus group discussion. As a token of appreciation for patients' time, they received a blood glucose meter that is equivalent to 12 Jordanian Dinar (approximately $£ 12$ ). Each focus group session lasted approximately 60-90 minutes. The focus groups were conducted in the Arabic language because it is the formal language in Jordan. Patients during focus group discussions were found to be relaxed and easily engaging with each other which facilitated collecting data to answer the research questions. In addition to participant observation and focus group, a semi-structured interview was used to collect data from HCWs and patients' family carers. The semi-structured interview is discussed now.

\subsubsection{Semi-structured interviews}

The interview is the most common type of qualitative data collection method, and it is commonly used to obtain data in ethnography. There are three types of interview: structured, semi-structured and unstructured interview (Bryman, 2012).

Structured interviews are composed of a predefined list of questions. All of the questions are asked precisely to all participants in the same way and in the same order. A structured interview is easy to administer, commonly used in survey research and it is also suitable if the sample size is large (Bryman, 2012). Additionally, it is used to minimise the interviewers' variations in conducting the interview and increases the accuracy of the results (Bryman, 2012). However, structured interviews only permit participants to report on a specific and narrow range of questions pre-determined by the interviewer and are little used to gain rich information (Gill, Stewart, Treasure, \& Chadwick, 2008). Structured interviews were not suitable to be conducted in my study because it would not achieve the purpose of this study to generate rich, in-depth information and uncover issues which may not previously have been reported.

In unstructured interviews, the interviewer asks questions in an informal mode. The interviewer has only a list of topics that should be covered during the interview (Bryman, 2012). The words and the order of questions may vary from interview to interview (Bryman, 2012). Unstructured 
interviews are suitable in the exploration of new research topics where little is already known (Morse \& Field, 1996). However, unstructured interviews are time-consuming and the lack of a predefined questions guide makes this mode of interview challenging to manage (Gill et al., 2008). Unstructured interviews were not suitable for this study because I had set objectives which needed to be explored and unstructured interviews might yield irrelevant answers outside of the scope of this study, especially when conducted by a novice researcher. Unstructured interviews might also take too long to conduct which would add a time challenge because of the availability of HCWs and patients' family carers. Additionally, there were particular issues I wished to explore, namely barriers and facilitators to good glycaemic control, to inform the development of a health promotion programme for patients and therefore this form of an interview was inappropriate for addressing my research aim and objectives.

The semi-structured interview is more commonly used in anthropology (Davies, 2008). Semistructured interviews are more flexible than structured interviews and more controlled than unstructured interviews (Gill et al., 2008). The researcher has to prepare an interview guide. According to Bryman (2012), the interview guide is a list of questions to be covered during the interview. In semi-structured interviews, the sequence of the questions in the interview guide can be adjusted, and new questions can also be added to the interview guide based on participants' responses (Bryman, 2012).

A semi-structured interview approach was chosen to use in this study. The semi-structured interview compared with a structured interview, allowed for a more in-depth understanding of issues rather than superficial and limited answers (Bryman, 2012), and enabled the generation of rich information focused on the topics related to my study rather than provide general and too much data as in unstructured interviews.

A debriefing session was conducted after each interview had been completed. Any misconceptions by participants were addressed during this debriefing session. As a qualified nurse and within my knowledge and experience I was able to address patients' and their carers' questions about their diabetes and glycaemic control and suggested they speak with their doctors. I also directed participants to the National Centre for Diabetes, Endocrinology and Genetics in Jordan for more information and support.

Semi-structured interview guides for HCWs (Appendix 11) and patients' family carers (Appendix 12) were developed based on the aim and objectives of the study, from reviewing the literature, from expert recommendations and informed further from the participant 
observation. Both interview guides focused mainly on the factors associated with poor glycaemic control levels among people with Type-2 diabetes and potential solutions to improve glycaemic control levels. To test the clarity and relevance of the initial interview guides, they were piloted with one nurse who provided formal direct care to patients with Type-2 diabetes and one patient's son who attended the medical appointment with his mother. Both of them agreed to take part in the pilot interviews and there were no changes made after piloting.

Health education materials in the centre were also reviewed in this study. Documentary review as a method of data collection is discussed now.

\subsubsection{Documentary review}

The documentary review was conducted through an analysis of the current diabetes education programme and diabetes educational leaflets that were provided in the centre. Documentary data were sampled purposively based on the aim and objectives of the study. I explored the educational information, appropriateness of educational materials for the Jordanian context, assessed the readability of educational material using a specific test and used Scott's criteria (1990) to evaluate the authenticity, credibility, representativeness and meaning which has been discussed in the sampling of documents section, and if the existing educational materials contained up-to-date information or not. In addition to the analysis of the educational materials, I gathered numerical information about patients' attendance in the educational sessions and the number of patients who partially or fully completed the educational programme. With participants' permission, all data related to patients' attendance were obtained from patients' files by the HCWs.

My study was conducted in a health care setting and in particular with patients. Ethical issues were taken into account in all phases of the study. The following section discusses the ethical considerations of this study.

\subsection{Ethical considerations}

The main ethical principles related to participants' rights include informed consent, anonymity, confidentiality and avoiding harm to participants (Bryman, 2012). Firstly, informed consent is discussed now. 


\subsubsection{Informed consent}

Informed consent (Appendix 9) was gained before any data collection from participants and before commencing the focus groups and semi-structured interviews. Potential participants were given a period of two weeks between being given the information sheet and being interviewed. Before starting the focus group session or interview, I described the purpose of the study and the nature of the interview or the focus group carefully. All participants were given the opportunity and the time to ask questions and all questions were answered before they were asked to sign the consent form. All the study participants were given an information sheet (Appendix 6,7 and 8) which contained information about the aim of the study, location and length of interview/focus group, and any potential risks to the participants. A participant information sheet was worded to be suitable for all participants. All participants were informed that they had the right to accept or refuse the audio recording of their discussion and interviews. Patients were also informed that participation in this study was voluntary and refusing to take part would not affect the quality of care provided to them.

Verbal approval was secured from the director of the health care centre to conduct participant observation in different areas in the centre such as the consultant's room, pharmacy, waiting area, nurse's room and the conference room. The director also approved me to attend any formally conducted educational session about diabetes in the centre and have access to the educational documents in the selected centre. A covering letter (Appendix 3) was requested by the director of the centre from the Directorate of Health in the Ministry of Health. A covering letter is an official document provided by the Directorate of Health to the researcher to facilitate his or her access and movement for conducting research in the selected centre. The letter was secured, I had an informal discussion with the director of the centre, and the approval was granted.

During participant observation in the consultant's room, I introduced myself to the patients and asked for their verbal permission to conduct observations. Ten patients who did not wish to be observed informed me, and I did not include them in the participant observation. During the observations, I conducted informal conversations with the HCWs, patients with poorly controlled Type- 2 diabetes and their family carers. During these discussions, I wore an identification badge stating that ' $\mathrm{I}$ am a PhD research student'. That enabled me to be introduced and identified by patients in different areas of the centre as a research student who 
was conducting a research study. I then posted a poster about the purpose of the study in the waiting area in the centre in a visible and readable manner to all patients (Appendix 15).

The second essential principle is anonymity which is discussed now.

\subsubsection{Anonymity}

In order to guarantee participants' anonymity, any quotes from focus groups, participant observation and interviews were assigned pseudonyms. All audio tapes were translated and transcribed by me, and only anonymised data were provided to my supervisors. A professional translation service in the UK was used to conduct the back translation of a sample of the transcripts (10\%) and who were bound to maintaining anonymity and confidentiality. The study setting was also anonymised. In addition to anonymity, the assurances given regarding confidentially are presented now.

\subsubsection{Confidentiality}

Information gathered about study participants and the study context was carefully stored and protected. Data were saved in my password protected computer, with hard copies in a locked cabinet in a locked office. All steps to ensure confidentiality were reported in the participant information sheet and consent form. The audiotapes from the focus groups and interviews were identified by numbers and not by names or job titles for HCWs. I transcribed the audiotapes in a private room and nobody heard the conversations to ensure confidentially. I ensured all study participants that the audiotapes would be destroyed after this study is completed. All focus group sessions and interviews were conducted in a quiet, comfortable and private room.

Before beginning, the focus group session ground rules were established to seek to preserve the confidentiality of the discussion between patients and all the focus group patients were asked to follow these rules. I explained that each patient's words would be shared with other patients in the room. Patients were asked to respect other participants' confidentiality and not to share any information with people outside the focus group. After I had read out the ground rules, I sought verbal agreement from all focus group patients to the ground rules and maintaining confidentiality. I also explained to the patients whilst I, and the FG assistant who assisted with the focus groups would maintain confidentiality, I could not guarantee that other patients in the focus group would do so. At the end of the focus group, again I emphasised respect for maintaining other patients' confidentiality during a debriefing session and all were given a debriefing sheet (Appendix 14). Besides the above ethical principles, ensuring that the 
study is free of any kind of physical harm to participants is crucial. Harm to participants is discussed now

\subsubsection{Harm to participants}

It was not foreseen that there would be any kind of physical harm to the study participants. However, it was recognised that the topic of the study might involve issues that could be sensitive to patients with poorly controlled Type- 2 diabetes and their family carers. Patients with poorly controlled Type- 2 diabetes and their family carers might feel stressed and upset talking about the reasons behind poor glycaemic control levels and their experiences living with Type-2 diabetes.

For the focus group patients, the presence of other patients who had poorly controlled Type-2 diabetes might provide support which might aid a change of thoughts and opinions during the discussion. There was also the FG assistant attending the focus group who did not participate in the discussion. In case of someone becoming upset, the audio recording would be stopped, and I would seek to console the individual. If the upset person would prefer to leave the room, then the FG assistant would be asked to accompany the individual to be consoled and supported without breaching the ground rules of confidentiality. Two patients felt upset while talking about their condition during the focus group discussions. A female patient in focus group 1 felt stressed whilst talking about the reasons behind high blood glucose levels, and we asked her if she wanted to leave the room, but she refused and stayed with us. Another female patient in focus group 6 also became upset and cried whilst talking about how she had diabetes, and the audio recording was stopped, and I sought to console her. I asked her if she preferred to leave the room and the FG assistant would accompany her, but she stayed. At the end of the focus groups, I ensured that these participants did not leave the centre feeling distressed. Indeed, the focus groups participants reported how they had enjoyed the opportunity to talk about their experiences and share their views.

I am also a registered nurse and a member of the Jordanian Nurses and Midwifery Council (JNMC). As a registered member of the JNMC, I am a qualified nurse with experience of consoling people at times of distress. I am duty bound to keeping any patient's information and personal data confidential and secure. I also had the contact details of sources of support such as the National Centre for Diabetes, Endocrinology and Genetics and diabetes support groups in Jordan. Additionally, as the focus group was conducted in the health care centre patients had access to further sources of support if required. 
For the semi-structured interview participants, in case someone might become upset, I would stop the interview and sought to console and support them. I also assured the participant that his/her participation would be kept confidential and pseudonyms would be used for all names to protect participants' identities. I also had the contact details of sources of support such as National Centre for Diabetes, Endocrinology and Genetics and diabetes support groups for patients' family carers and the Occupational Health Department for HCWs. The last ethical principle is ethical approval which will be discussed next.

\subsubsection{Ethical approval}

Ethical approval was obtained from the College of Human and Health Sciences Ethics Committee at Swansea University in the United Kingdom (Appendix 1). This study was conducted in a comprehensive health care centre managed by the Primary Care Department of the Ministry of Health in Jordan. Ethical approval from the Ministry of Health (Appendix 2) and permission from the director of the selected health care centre were also gained.

The next section discusses the process of data handling and data analysis.

\subsection{Data handling and data analysis}

\subsubsection{Data handling}

The focus group sessions and semi-structured interviews were audio recorded. All were transcribed by me, which helped me to review the discussion and compare it with the handwritten notes provided by the assistant for verification. I organised and prepared the raw data such as focus group and interview transcripts, diary notes and participant observation notes for the analysis process. The researcher has to organise and sort the collected data physically to different boxes with numbers or uploaded electronically to computer files (Mason, 2002). I uploaded the transcripts and field notes to separate computer folders and files based on the focus groups with patients, interviews with $\mathrm{HCWs}$ and family carers and participant observation notes. A sample $(\mathrm{n}=2)$ of the Arabic transcripts translated into English by me was back-translated by a bilingual translator to verify the accuracy of my translation. The translator was asked to sign an agreement to preserve the confidentiality of the transcripts. Two copies of each transcript were made. I read and reread all written transcripts to gain a sense of the 
whole content and made notes. To further ensure that the meaning of the actual Arabic context was preserved in the English language, I performed coding and analysis of the English translated transcripts, whilst listening to the original Arabic audio-recordings of the focus groups and interviews. Also, during the process of translation, I made sure that I conveyed the actual meaning of what participants said in the interviews and focus groups.

My study's aim was to seek to understand the meaning behind people's health behaviours and their words. Thereby, I followed Mason's (2002) recommendations which qualitative researchers should read the written transcripts and field notes during the data analysis process literally, interpretively and reflexively. A literal reading of all transcripts was conducted to provide me with insight and understanding of the data (Mason, 2002). Interpretive reading was also sought to explore and gain the deep meaning behind people's words and comments about their life experiences with diabetes. In interpretive reading, the researcher represents people's interpretations, or summarises how people make sense of the social world, or constructs the meaning of data (Mason, 2002). Additionally, I undertook reflexive reading which enabled me to understand the effect of my personal perspectives and my role as the researcher on data generation and data analysis (Mason, 2002).

I immersed myself in the collected data by being a part of the patients' community which enabled me to have a better understanding of patients' perceptions, experiences and behaviours. The researcher should immerse and engage himself in the qualitative data which enables him to be familiar with the underlying meanings of it (Bryman, 2012). After reviewing the data, a data reduction process began in order to focus on meaningful and significant data that was related to the research aim and objectives. In the next section, I provide the reader with information about the data analysis process.

\subsubsection{Data analysis}

I started the data analysis process as soon as data collection commenced. Creswell (2014), states that data analysis is an ongoing process and should be conducted at the same time as data collection and writing study findings. Qualitative research often yields too much data, and thereby data analysis should be started early with the first data collection such as analysing the first transcribed interview (Silverman, 2011). In contrast, data analysis in quantitative methods should only be started after completing the data collection. Thematic analysis was employed to analyse the qualitative data. According to Bryman (2012), thematic analysis is " a term used in connection with the analysis of qualitative data to refer to the extraction of key themes in 
one's data' (p. 717). Thematic analysis is one of the most common approaches of analysis in qualitative methods (Bryman, 2012). I conducted interpretive, latent thematic analysis to identify the subjective meaning beyond patients' words and behaviours. The latent thematic analysis is a strategy that seeks to extract the underlying meanings of written and spoken words and is a common approach among constructionists (Braun \& Clarke, 2006). Braun and Clarke (2006), define a theme as something crucial and important in the researcher's data related to the aim and objectives of the study. Initial codes, categories, subthemes and themes were generated from reading and reviewing the transcripts and field notes many times (Bryman, 2012). An ethnographic design requires a full description of the research setting and participants to provide context followed by an extraction of the key themes from data (Creswell, 2014).

During the data analysis process, I moved backwards and forwards in a 'recursive process' between my collected data as needed to give me a comprehensive picture of people's meanings (Braun \& Clarke, 2006, p. 86). Additionally, I employed 'inductive and deductive data analysis' (Creswell, 2014, p. 186) strategies in which I extracted the themes and subthemes from the collected data inductively. Then deductively, I returned to the database to find out whether these themes needed more supporting evidence or gathering more information regarding the generated themes. To support the data analysis process, all transcripts were uploaded into the qualitative data analysis software pack NVivo 10, which facilitates storing, searching, categorising and coding of the data (Creswell, 2014). I grouped relevant words, sentences and phrases to create initial codes. Initial codes were generated manually by using Microsoft Word. I grouped the initial codes and the supporting data for each code. Initial codes only reflected what the participants actually said in this study without any interpretation by me, so I did not miss out on coding data. 87 initial codes were generated. Then, I reviewed all the created codes, and during that, more codes were added or dropped. The coding process is used in ethnographic data analysis to develop full descriptions of the setting, people and activities (Creswell, 2014). Then I summarised and developed concepts and categories by grouping these initial codes into categories and subcategories. Significant categories relating to the aim and objectives of this study were grouped together to generate themes, and these themes were labelled and connected to each other. These themes were compared with the existing literature. I provided in appendix 20 an example of how the key theme of 'Influence of the Jordanian socio-cultural context on patients' diabetes management plan' was derived from the initial codes and categories. Sociodemographic data were analysed manually using descriptive statistics including 
frequencies, mean, median, standard deviation and range (Creswell, 2014). In the next section, I will discuss the criteria that were used to evaluate my research process and findings.

\subsection{Methodological rigour}

Creswell (2014), states that demonstrating validity and reliability in qualitative research is different from quantitative research. The notion of validity in quantitative research is usually referred to measurement which is difficult to be applied to the qualitative approach (Bryman, 2012). Hammersley (1992) noted there was general disagreement among qualitative researchers about which criteria should be used to evaluate qualitative research. Some researchers use criteria derived from quantitative research to evaluate qualitative research while others have argued that qualitative research should not be evaluated using quantitative research evaluation criteria (Bryman, 2012). Other qualitative researchers offer alternative methods to evaluate qualitative research such as Lincoln and Guba. Lincoln and Guba (1985) proposed trustworthiness and alternative criteria for judging the quality of qualitative research; these are credibility, transferability, dependability and confirmability. In contrast, Hammersley (1992) veers between using quantitative research criteria and alternative methods to evaluate qualitative research (Bryman, 2012). Hammersley (1992) argues for "subtle realism" (p. 5), that recognises an independent external reality but also that different representations of reality exist (degrees of reality), and all are equally valid. Hammersley (1992) also introduced the concepts of validity and relevance as criteria to evaluate qualitative research findings. In this study, Lincoln and Guba's evaluation criteria (1985) together with Hammersley's evaluation criteria (1992) were used to evaluate my study findings, which I found to be convincing. Lincoln and Guba's evaluation criterion is now discussed.

\subsubsection{Lincoln and Guba's evaluation criteria (1985)}

Lincoln and Guba (1985) proposed credibility, transferability, dependability and confirmability for judging the quality of qualitative research.

Credibility is defined as confidence in the truth of research findings and is equivalent to internal validity (Bryman, 2012). Credibility is enhanced by using triangulation, prolonged engagement and the use of a reflexive journal (Lincoln \& Guba, 1985). Triangulation is a strategy that uses a combination of more than one research method or multiple theories, or different data sources in the study of the same social phenomenon (Silverman, 2011). If several methods of data 
collection yield similar issues and themes then validity can be claimed in the study (Creswell, 2014). Triangulation can also be undertaken by seeking information from different sources or using multiple investigators (Lincoln \& Guba, 1985). Triangulation in this study was sought in two ways; firstly, by applying four methods of data collection which were participant observation, focus group, documentary review and semi-structured interviews. Secondly, by seeking data from different groups of participants (patients with poorly controlled Type-2 diabetes, their family carers and HCWs) which provided rich, in-depth data. For example, participants from all groups reported that the discontinuity of care was a challenge to adherence to patients' management plan.

Prolonged engagement with participants and repeated observations have been argued to increase the validity of the claims (Lincoln \& Guba 1985; Creswell 2014). However, prolonged engagement in itself does not necessarily enhance credibility, and it is the extent to which the researcher develops rapport and trusting relationships with participants, learning about their culture and testing for misinformation introduced by researcher or participants (Lincoln \& Guba 1985). Prolonged engagement with participants and repeated observations enabled me to generate rich data, gain insight and familiarity with the setting. This study involved scheduled observations of patients with poorly controlled Type-2 diabetes in different areas in the selected health care centre that occurred over a four month period. However, a disadvantage of prolonged engagement in the setting is the possibility of going native and the potential risk of developing an intimate form of relationships with participants (Gold, 1958). I was able to develop a good rapport with participants, so they felt comfortable to engage with me but was aware not to immerse myself too deeply as an insider otherwise I might lose my outsider perceptive (Giddens \& Griffiths, 2006). Having regular supervision with my supervisors and keeping a reflexive journal enabled me to avoid going native and maintain a critical stance to what I observed and what participants said to me whilst also developing a rapport with participants. My use of a reflexive journal to enhance rigour is discussed later under the section titled confirmability.

Transferability is an alternative criterion to external validity and occurs when the research findings can be applied to other situations (Bryman, 2012). Lincoln and Guba (1985) suggest: "the degree of transferability is a direct function of the similarity between the two contexts" (p. 124). Transferability can be made if the researcher provides sufficient information relating to the study context which shows similarities with two different contexts (sending and receiving contexts) (Lincoln \& Guba, 1985). The reader can judge transferability when the researcher 
provides a "thick description" of the study (Lincoln \& Guba, 1985, p. 125). Muecke (1994) proposes a thick description as a criterion to evaluate ethnographic research. Thick description is known as a rich account of the studied culture and includes in-depth information about the social setting, events, behaviours, meaning, people and context (Bryman, 2012). The researcher should be precise regarding which information is relevant to be included in the thick description as too much data are often generated from qualitative research. Bryman (2012, p. 401) states that the researcher has to avoid 'descriptive excess' which means the researcher provides irrelevant information as the thick description which may overwhelm the account. Thick description enables other readers to make judgments about the potential to apply all or part of my study findings to other contexts, settings or people (Schwandt, Lincoln, \& Guba, 2007) or to the same context at a different time (Lincoln \& Guba, 1985). Transferability can only be made when the researcher has sufficient information about the two different contexts (Lincoln \& Guba, 1985). My responsibility is to provide sufficient detailed information about the context of the study and the findings so that the reader can judge the transferability of the study findings. However, I have to ensure that the anonymity of the study participants and setting are protected.

Generalisation is used in a limited way in qualitative research due to multiple factors such as small sample sizes, lack of random sampling and inability to generalise the study findings to other contexts (Bryman, 2012; Silverman, 2011). Despite that, the primary aim of the ethnographic research is to describe and explain what is observed in the particular study setting, so generalisations should be sought in an ethnographic study to claim relevance of findings (Hammersley, 1992). Mason (2002) also supports Hammersley's view which proposes that qualitative research should provide some forms of generalisation. There are two forms of generalisation in the qualitative approach, theoretical and analytical, which have much the same direction (Bryman, 2012). The study findings and inferences may be generalised theoretically (Mason, 2002) to other patients with poorly controlled Type-2 diabetes in similar contexts. Then claims for relevance and contributions to the cumulative body of knowledge can be made.

Dependability, according to Bryman (2012), is the stability of research findings over time and is a substitute for the positivist notion of reliability. Social reality from a social constructionism paradigm is negotiated, constructed and reconstructed by people (Bryman, 2012). Indeed, social reality is ongoing and changing and is not static; therefore, reliability in a positivist stance is not applicable to social science (Silverman, 2011). However, I seek dependability in 
my research to enhance the rigour of my study findings. In order to establish dependability, the researcher's decisions should be clear and transparent so that another researcher would be able to replicate the study (Noble \& Smith, 2015). Dependability will be demonstrated by providing an audit trail and through inter-rater reliability. An audit trail is a record of all the research phases, activities and decisions made by the researcher (Bowen, 2009). To provide an audit trail, the following data are kept: raw data, data analysis, decisions made, fieldwork notes, reflexive journal, study findings and final report, personal and methodological notes, and interview transcripts (Bryman 2012; Lincoln \&Guba 1985). In addition to the audit trail, an external researcher may review whether the research findings, interpretations and conclusions are supported by the research data (Cohen, Manion, \& Morrison, 2011).

There is a strategy called inter-rater reliability which was used to ensure the rigour of the data analysis process. Armstrong, Gosling, Weinman, and Marteau (1997) define inter-rater reliability as a strategy in which data are coded by different researchers or coders, and this coding process can be assessed by the degree of agreement among the researchers so the claim for reliability can be made. This strategy mainly relates to the coding process in the statistical analysis in quantitative data analysis, and it is used to verify consistency of the extracted codes from structured interviews and questionnaires between different coders (Bryman, 2012). Interrater reliability was used in my study during the data analysis process. A sample of data extracts $(n=2)$ from the focus groups and individual interviews transcripts was given to my supervisors to be read, analysed and coded according to the coding framework which I had developed. My supervisors share the same professional background (nursing) which means the selected transcripts were analysed by three people with the same professional background to ensure the reliability of the study findings. They were asked to extract the main codes and categories in these transcripts as the thematic analysis was used in this study. If similar codes and categories are identified among us, then reliability will be claimed in this study. $80 \%$ of inter-rater reliability was achieved and agreed by us.

Confirmability is concerned with the extent to which the research findings are derived from study participants and not from the researcher's interests (Lincoln \& Guba, 1985). Confirmability in this study will be achieved by using triangulation, an audit trail and maintaining a reflexive journal to ensure this study's findings are grounded in the data (Lincoln \& Guba, 1985). 
To ensure confirmability in this study, deviant cases were taken into account during data analysis and the reporting of the findings. Deviant or negative cases are a group of episodes that refute the general supposition regarding the studied phenomenon (Morse \& Field, 1996). Deviant, negative or disconfirming cases are atypical cases (Morse \& Field, 1996) and including them in data analysis provides another insight and further exploration of reasons behind poorly controlled Type-2 diabetes. For instance, when I asked patients questions to explore which topics they thought needed to be covered in any future health education sessions, one male patient perceived that the doctors were more aware of a patient's health care needs. None of the other patients reported that health education programmes should be designed by the doctor based on individual needs.

My use of triangulation and an audit trail to enhance the rigour of the study have been previously discussed in the sections on credibility and dependability. A reflexive journal includes daily diaries, methodological decisions made, biases, experiences, values, a full description of all the events that happen, and personal reflections (Lincoln \& Guba, 1985). Maintaining a reflexive journal claims to enhance not only confirmability but also credibility, transferability and dependability (Lincoln \& Guba, 1985). I have adopted a reflexive approach in all stages of my study and report all decisions which were made in the conduct of this study. For instance, in the sampling of areas for participant observation, the consultants' rooms were not considered in my initial selection of areas. However, from observing the clinics, it became apparent that having the opportunity to observe consultations between the consultants and their patients would enable me to see whether health promotion was offered and delivered during the consultations. Prolonged engagement enabled me to develop rapport and trusting relationships with the consultants who, with the permission of patients, invited me to observe their consultations

Hammersley (1992) proposes the concepts of validity and relevance as criteria with which to evaluate the rigour of ethnographic research findings. These criteria are discussed now.

\subsubsection{Hammersley's evaluation criteria (1992)}

Validity is mainly used in quantitative approaches as a criterion to claim and evaluate research findings (Silverman, 2011). However, Hammersley (1992) argued that qualitative research findings should be valid and relevant to human beings. Validity means " truth." (Hammersley, 1992, p. 69). The account is considered valid if it represents attributes of the social phenomena 
accurately. However, we can never be certain about the truth of any account because we do not have completely reliable access to social reality. Therefore, validity must be judged based on the adequacy of the evidence that is used to support the claims (Hammersley, 1992).

Hammersley (1992) proposed three considerations to evaluate the validity of qualitative research. Firstly, when the researcher provides a claim which is not sufficiently plausible, more supporting evidence should be provided to enable assessment of the claim's credibility. The supporting evidence needs to be sufficiently plausible and credible and be able to convince multiple audiences. Secondly, central or core claims such as the main findings and critical decisions need more evidence from the researcher than marginal claims. Thirdly, evidence must refer to the claims made and as such different kinds of evidence and resources are required to meet different types of claim.

Lincoln and Guba (1985) view credibility as being a crucial criterion to assess the trustworthiness of the study findings. However, the credibility of research findings and interpretations cannot be well-established without submitting the research findings to the people studied for confirmation (Lincoln \& Guba, 1985). Credibility should first be judged by research participants, and without their agreement, the credibility of research findings is threatened (Lincoln \& Guba, 1985). This strategy is known as member checking or respondent validation, and it is another strategy proposed to maximise the accuracy of study findings by taking the final report or specific descriptions or themes or interpretations back to participants to verify they are accurate (Creswell, 2014). However, conflicts of interest may vary among the participants (Hammersley, 1992; Lincoln \& Guba, 1985). Hammersley (1992) does not agree with Lincoln and Guba's stance regarding the judgement of the credibility of research findings based on respondent validation. Hammersley (1992) stated that credibility requires precise interpretation and should not be judged by the people studied. I agree with Hammersley's perspective regarding the credibility of my research findings and therefore did not seek respondent validation. Study participants may also be unaware of the key features of the social phenomenon particularly if it has future long-term consequences such as poorly controlled Type-2 diabetes and applications, not immediate ones (Hammersley, 1992). However, Hammersley (1992) has argued that respondent validation can be used as an essential source of information, but it cannot be used as a tool to assess the validity of claims. Member checking was not used as a strategy to assess the validity of this study findings for the previous reasons stated. Additionally, asking study participants (especially the focus group patients) to regroup would have been potentially difficult and time-consuming for patients. 
Hammersley (1992) also proposes relevance to human interest as an essential criterion to evaluate ethnographic research products. According to Hammersley (1992), there are two features of relevance: the importance of the topic which has to be within the substantive field and the contribution of the research findings it makes to the existing literature. I chose poorly controlled Type- 2 diabetes as a research topic because of the high prevalence rate of poorly controlled Type-2 diabetes, related complications and lack of published literature in Jordan. Research should be able to produce general, relevant findings rather than the solution to particular issues (Hammersley, 1992). Ethnographic research findings have to address the expectations of different potential audiences such as patients, the research community, policy makers and practitioners. Hammersley (1992) argues that fellow researchers are the immediate and most important audience for research findings.

In my opinion, patients with Type- 2 diabetes, particularly those with poorly controlled diabetes and practitioners, are the most important audience for my research rather than the research community. The findings of this study will specifically be used to improve the future care provided to patients with Type-2 diabetes, inform the development of an educational package for patients with Type- 2 diabetes and inform policy and practice

Ethnographic findings should also have relevance for policymaking (Hammersley, 1992). Hammersley (1992) recommended that findings from multiple studies may have a better influence on policymakers compared with particular study findings. Ethnographic findings can be relevant to a practitioner audience in a direct/indirect way, and it is anticipated that the findings from my study will have direct relevance for the HCWs and the health care setting in which the study was conducted. Ethnographic products may influence other research outcomes and have direct relevance or future long-term applications, not just immediate ones (Hammersley, 1992).

Throughout the presentation of the findings and discussion in this thesis, data extracts from a range of participants have been provided to support the claims made. Such data extracts can aid the reader in reaching decisions about the plausibility and credibility of my findings and interpretation, and that should be judged based on the adequacy of the evidence that is used in support of the claims (Hammersley, 1992). In the next section, I will discuss reflexivity and the reflexive approach that I followed during my research. 


\subsection{Reflexivity}

Ethnographers have begun to be more involved with participants in the study settings and gradually shifted away from the positivist stance since 1970 (Davies, 2008). These changes in ethnographic research led to incorporate reflexivity (self-recursive) as an important part of the ethnographic research process (Davies, 2008). Reflexivity allows the researchers to bring their own backgrounds, experiences and thoughts to the study (Creswell, 2013). Reflexivity also includes a regard for the influence of the researcher's background and role in data collection and interpretations (Creswell, 2013). Davies (2008) defines reflexivity as "a turning back on oneself, a process of self-reference" (p. 4). Reflexivity does not only refer to the influence of the researcher on data but also to the active role of the researcher in constructing and interpreting data (Davies, 2008).

The nature of my ethnographic research in which I had to immerse myself, talk to participants and be a part of the participants' setting necessitated that a reflexive approach was adopted. Bryman (2012) states that the qualitative researcher is a part of the participants' social world and is the instrument which gathers data from participants. Excluding the effect of the researcher's presuppositions, expectations and thoughts about participants' social world is an unfeasible aim for researchers (Hammersley \& Atkinson, 1995). Thereby, the notion of reflexivity should be acknowledged and taken into account in all stages of my study starting from choosing the topic about poorly controlled Type-2 diabetes to writing my study findings (Davies, 2008). Reflexivity is a broad, comprehensive notion which includes the whole research process. Reflexivity is an ongoing process of critical analysis and interpretation which should be incorporated in each stage of the research process (Guillemin \& Gillam, 2004).

Reflexivity has enabled me to acknowledge my taken for granted professional and personal values and experiences. I had developed and incorporated reflexivity into my research from the beginning with my interest in conducting research about Type-2 diabetes. The objectives of this study were derived from the existing literature and stimulated by my interest in the reasons for poorly controlled Type-2 diabetes in Jordan. Hammersley and Atkinson (1995) address that social events, professional and personal experience, surprising facts and literature are examples of stimulants in conducting research. My professional experience of being a nurse and having worked with patients with Type-2 diabetes in Jordan stimulated my interest in Type-2 diabetes. The research topic which is within the area of my professional expertise and my personal characteristics such as having three family members with Type-2 diabetes gave me insight into 
factors that may impact on the management of Type-2 diabetes. Throughout the write up of the thesis I have sought to demonstrate and make explicit to the reader how my personal and professional background, values and beliefs have influenced the conduct of the entire study.

The advantages of declaring my profession as a nurse and sharing similar experiences with health care workers and the same cultural background with all participants enabled me to gain a deeper understanding and build a better rapport, access and trust with study participants (Dwyer \& Buckle, 2009). Ethnographic research is currently conducted by people of the same culture or similar cultures rather than by people from different cultures as in early ethnographic studies (Pellatt, 2003). I have had the clinical background in an Intensive Care unit in one of the large hospitals in Zarqa city in Jordan. The primary care setting was interesting for me as it is different from hospital settings. The system of care, the severity of cases, patients and context are examples of differences between hospitals and primary and comprehensive care centres.

While I was conducting my research, I was not a complete insider or a complete outsider. An outsider researcher is defined as the researcher who is not a member of the cultural group under the study. On the other hand, the individual who has intimate knowledge of the population of the study and is part of the culture is known as an insider researcher (Dwyer \& Buckle, 2009). My status was both an insider and an outsider researcher. Being part of the participants' culture under the study poses challenges. Insider researchers may face difficulties to create enough distance between themselves and their own cultures. Sharing the same experiences with participants may also influence the researcher's perceptions (Dwyer \& Buckle, 2009). Though I am part of the culture, I might not understand subcultures and people are not homogeneous, and they also have different perspectives. Culture consists of subcultures, and they share common values and beliefs. However, each subculture has unique features (Helman, 2007). Culture is in an ongoing state of adaptation and revisions, not as a static event (Helman, 2007). I was an insider in the sense that I am of Jordanian origin, that I speak Arabic and as a professional nurse to some extent, I was an insider with the HCWs. However, I was also an outsider as I have not worked in the comprehensive care centre or had not lived or worked in Jordan for the last eight years. In many respects being both an insider and outsider was beneficial as I could speak the language, put participants at ease and easily gain rapport whilst also maintaining a critical stance to what I observed and heard as I was not familiar with the primary care centre setting. 
Maintaining a reflexive approach is an important aspect in the ethical process of the whole research study which enables the researcher to be aware of potential ethical issues and assure good quality research (Hammersley \& Atkinson, 1995). Being a reflexive researcher, I wrote in the ethical consideration section how the focus group and interview discussions might involve issues that could be sensitive to patients with poorly controlled Type-2 diabetes and their family carers before any actual research was conducted, and considered how I would respond to these issues appropriately. During the actual focus group or interview, I was better placed and prepared to be aware of emergent ethical issues as they happened with two patients while conducting focus groups.

At the data collection process, I was aware of the factors that might influence the nature of the collected data such as the interactions with participants, posing questions, listening to participants and preparation of study settings for focus groups and interviews. Following each focus group or interview, I recorded my personal reflections on the conduct of interviews and focus groups. Whilst conducting participant observations, I also wrote up the field notes observations after every visit where I conducted observations to enable me to have a full history record and to avoid missing valuable data. During the data analysis process, I adopted strategies for enabling reflexivity through reflexive reading which enabled me to understand the effect of my personal perspectives and my role as the researcher on data generation and data analysis (Mason, 2002).

When the ethnographer starts writing the final product, he/she can be reflexive in the style of writing to meet knowledge and expectations of multiple readers such as study participants, published journals, students and university lecturers (Hammersley \& Atkinson, 1995). In order for the reader or audience to judge how my beliefs and values have influenced the entire research study, I have adopted a reflexive approach throughout. This will enable the reader to judge whether this study's findings are credible and plausible or not.

To aid interpretation of the findings and discussion chapters which follow, an overview of the theories which have been drawn upon to aid interpretation of the data and which became apparent during data analysis are now presented. 


\subsection{Overview of Theories}

This section provides a description of the theoretical perspectives that were considered to offer appropriate analytical insights into my data. A number of theories have been drawn upon to aid understanding of the factors which influenced poor glycaemic control amongst patients with Type-2 diabetes. In this section, I provide a critical overview of the socio-ecological health model (SEM) (McLeroy, Bibeau, Steckler, \& Glanz, 1988), the medical model of health care, the health belief model (Rosenstock, 1974) and locus of control (Rotter, 1966). The socioecological health model is discussed now.

\subsubsection{Socio-ecological model (SEM)}

After completion of initial data coding, theoretical perspectives were considered to aid understanding of the emergent findings. The socio-ecological health model (McLeroy et al., 1988) was considered as a main overarching model during data analysis. Public health professionals need a theoretical framework to identify and recognise the factors from different aspects affecting patients' health (Institute of Medicine, 2003) which is consistent with the principles of the socio-ecological health model. Theoretical frameworks assist the researchers in exploring and understanding the phenomenon of interest and the reasons behind it (Parker, Baldwin, Israel, \& Salinas, 2004).

The socio-ecological model has enabled me to gain insight into the collected data from the various groups of participants and therefore aid understanding of the factors associated with poorly controlled Type-2 diabetes phenomenon in Jordan from multiple determinants. Additionally, my findings identified the importance of the factors beyond the individual levels regarding social, cultural, organisational and policy influences. The SEM enabled the complexity of influences on patients' decision making and behaviours to be understood. According to Hammersley (1992), one of the two crucial features of relevance is the contribution the research findings makes to the existing literature and theoretical development. Therefore the SEM has provided a main theoretical background for aiding understanding of my research findings.

The socio-ecological model (McLeroy et al., 1988) is defined as a model of health promotion that incorporates multiple factors influencing patient's health by linking these influences with each other (Institute of Medicine, 2003). The findings of this study suggested that influences on patients' diabetes management occurred at multiple levels rather than one level. This suggests that patient's health behaviours can be explained from multiple levels of influence as 
each one of these levels contributed to the development of high BG levels among the patients in my study. The SEM was used as an overarching model that aid understanding of the overall findings of this study. The SEM emphasises multiple levels of influence on an individual's health and illness. An individual's health is examined at several levels of analysis (physiological, psychological, interpersonal, organisational, institutional and communal) (McLeroy et al., 1988). In the SEM, an individual's health status is also influenced by multiple circumstances found at different levels of the social environment (McLeroy et al., 1988) which suggests that individual's health can be understood from a holistic perspective. Integrating knowledge and research methods drawn from several different fields is needed for the design, implementation and evaluation of effective health promotion interventions (Stokols, Pelletier, \& Fielding, 1996).

The work of Bronfenbrenner (1977) was developed by McLeory, Bibeau, Stickler and Glanz (1988) who proposed the socio-ecological model (SEM) for health promotion based on concepts derived from the work of Urie Bronfenbrenner (1977). Bronfenbrenner (1977) from which McLeroy et al.'s model is derived proposed four levels of influence on an individual's behaviour; micro, meso, exo and macrosystem. The microsystem refers to an individual's relationships and interactions with immediate family and friends while mesosystem encompasses the interactions within various settings such as the workplace. The exosystem refers to forces within the larger social system, and macrosystem consists of cultural values, beliefs and social norms such as serving a particular kind of food during social gatherings and occasions (McLeroy et al., 1988; Glanz, 2002).

The socio-ecological model of health behaviour identifies five levels of influence with strategies that are linked, overlapped and interrelated with each other (McLeroy et al., 1988). These levels with examples from my study are illustrated in Figure 6. In the socio-ecological model, there is no separation between the individual and the social context and the relationship between the individual and the surrounding environment is reciprocal which influences health outcomes (McLeroy, Norton, Kegler, Burdine, \& Sumaya, 2003). Behaviour is not only a result of knowledge, attitudes and values of the individual but also it is the outcome of social values, interpersonal relationships and the wider community (McLeroy et al., 2003; DiClemente, Crosby, \& Kegler, 2009). Therefore, in order to aid understanding of an individual's health behaviours, the sociocultural influences and values should be considered during the behavioural changing process (McLeroy et al., 2003; DiClemente et al., 2009). 


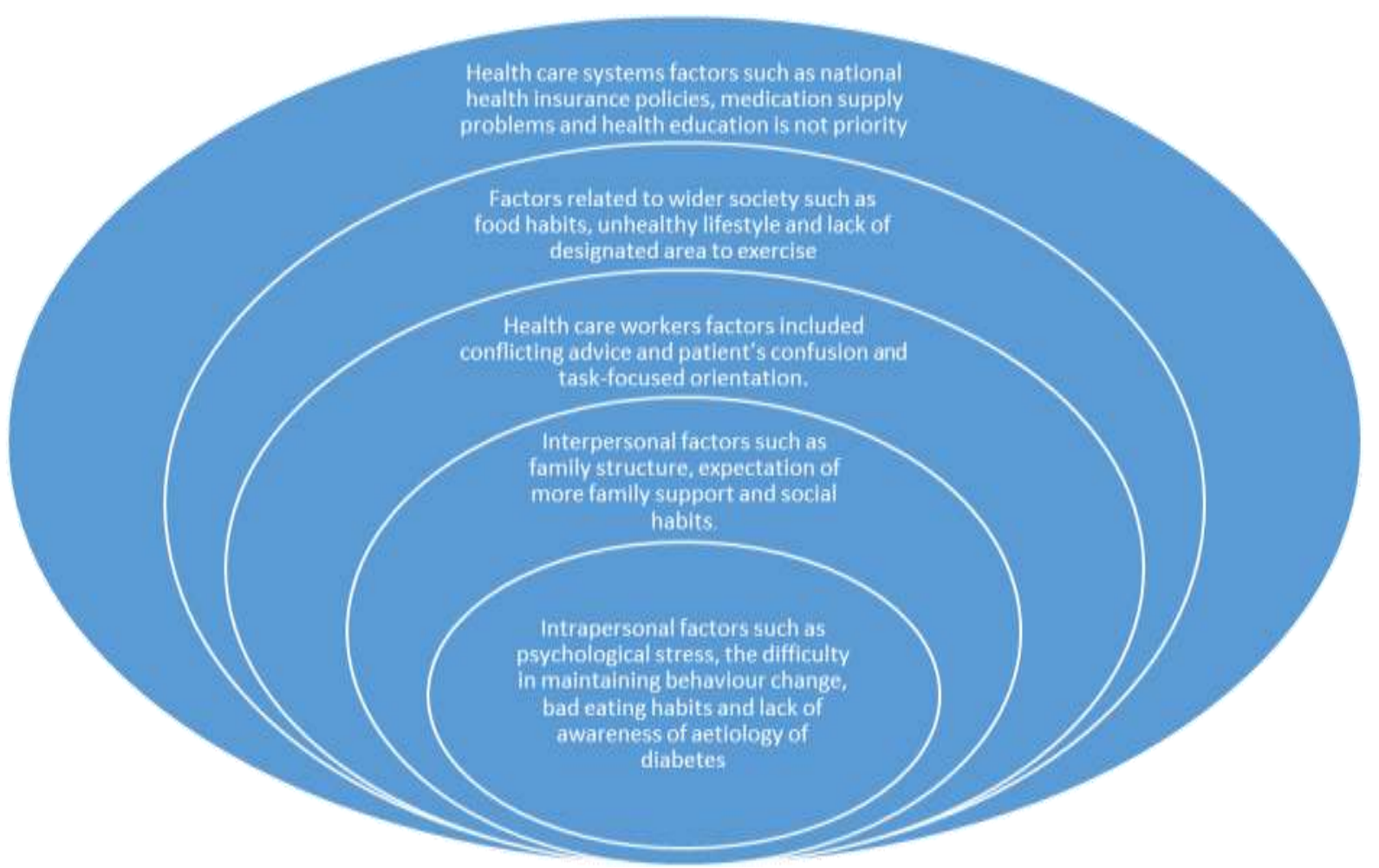

Figure 6 illustrates levels of influences in the socio-ecological health model with examples from my study findings.

The traditional model of the host, agent and environment roles is based on socio-ecological concepts (McLeroy et al., 1988). This communicable diseases model was utilised to study and analyse the lifestyle factors related to chronic conditions such as obesity and physical inactivity (McLeroy et al., 1988). The epidemiological model emphasises cause-effect relationships and individual's risk factors. Epidemiologists have recognised the importance of including the sociocultural factors which impact on an individual's health status as determinants of health (Richard, Gauvin, \& Raine, 2011). The meaning of health is not only embedded in the individual alone but also results from interactions with the surrounding environment (Stokols et al., 1996; McLaren, 2005). The socio-ecological perspective of diabetes self-management behaviours also goes beyond the ability of an individual to control his/her behaviours by incorporating skills of individuals within the health services and support from social relationships and the wider environment (Fisher et al., 2005). The environment in my study referred to the social environment of family, friends and the culture of the Jordanian society as well as the policy environment of the health care system and the health care centre. 
The SEM is viewed as an overarching model that shares concepts with system theory and social cognitive theory (McLeroy et al., 1988; Stokols et al., 1996; Glanz, 2002). The SEM shares features with system theory (Bertalanffy V, 1968) regarding the interaction between the individual and social environment incorporating bidirectional influence, multi-influenced and overlapping factors (Stokols et al., 1996). In system theory, a system is composed of different parts, and these parts are interrelated and interconnected with each other in a dynamic process (Bertalanffy V, 1968). The physical, mental and social health of individuals are influenced by a variety of physical and social environmental factors. The individual-environment relationship is characterised as a mutual influence in which the surrounding environmental factors directly influence individuals' health and, concurrently, the individuals in settings modify the healthiness of their surroundings through their individual and collective actions (Bertalanffy $\mathrm{V}, 1968)$. System theory was applied to aid in understanding how adherence of patients to their treatment plan is affected by factors related to different parts such as the health care system, the health care centre and HCWs. Medication supply problems in the health care system affected patients' adherence to their management plan in this study.

The socio-ecological model also incorporates concepts derived from social cognitive theory regarding the influence of sociocultural variables on an individual's health (Glanz, 2002). Social and cultural norms have a cumulative influence on an individual's health and behaviour (McLeroy et al., 1988). Social wellbeing is also an essential component of an individual's health (WHO, 1948). However, the majority of interventions and health promotion programmes have placed more focus on targeting the individual (e.g. lifestyle changes) and at the interpersonal level than aiming to modify the social norms and values (McLeroy et al., 1988; Glanz, 2002; Golden \& Earp, 2012). Moreover, health care providers are more familiar with individualbased interventions such as health education than multilevel interventions such as social and policy modifications (Glanz, 2002).

Social connectedness is strong in Jordan and individuals are more influenced by social norms when strong social ties exist among members of the society (Zahran, 2011). Additionally, DiClemente et al. (2009) pointed out that family and close friends (proximal effect) have a major influence on an individual's behaviour due to the frequency of contact and the nature of the relationship. The local community can be either a barrier or a facilitator for promoting and maintaining a healthy lifestyle (DiClemente et al., 2009). For instance, the availability of fast food restaurants, large portion sizes and physical exercise barriers in society influence an individual's health behaviours (Glanz, 2002). The socio-ecological model also expands to 
incorporate factors related to health care facilities and health care workers within these facilities as determinants of a patient's health status. Health care providers can go beyond providing care for patients by supporting patients to modify health behaviour (Peek, Ferguson, Roberson, \& Chin, 2014). According to the socio-ecological model, culture has a major impact on an individual's health behaviours. There are two main cultural environment domains; affective domain such as values, religion and norms which inform values and the cognitive domain such as laws, media and policies that inform knowledge (DiClemente et al., 2009).

There is a little known about utilising the SEM in Jordan and the Arab countries, which may account for health care providers being less familiar with multilevel interventions (Glanz, 2002). I have not identified any previous research which has applied the SEM to aid understanding of the factors influencing glycaemic control among patients with Type-2 diabetes in Jordan. The socio-ecological health model has been utilised as a theoretical framework by Mabry, Al-Busaidi, Reeves, Owen, and Eakin (2014) to identify the barriers and facilitators to physical activity from the perspectives of 10 Omani public health managers. The method of data collection was an in-depth semi-structured interview, and the interviewees were selected purposefully. All the interviews were conducted in the English language though all participants were Arabic speaking, so participants' responses were more likely to be limited (Mabry et al., 2014). This study's findings revealed four main categories of influences affecting physical activity in Oman. Intrapersonal factors such as lack of motivation, awareness and time; social factors such as taking part in physical activity for women outside is difficult and lack of a culture of exercise; physical environments such as hot weather and limited designated places for exercise; policies such as ineffective health communication and limited resources. The main advantage of this study was expanding the scope of interest by encompassing other social, environmental and political determinants of health (Mabry et al., 2014).

The socio-ecological model has some practical limitations regarding how socio-ecological interventions can foster and sustain community health promotion programmes and changes (McLeroy et al., 2003). Also, HCWs are more familiar with intrapersonal interventions such as education and the predominant theories in the literature, for example, the health belief model (Rosenstock, 1974), the theory of planned behaviour (Ajzen, 1991) and social cognitive theory (Bandura, 2001) have an individual orientation by changing individual motivation to stimulate behaviour change (Golden \& Earp, 2012). Application of multilevel strategies requires incorporation from different sectors and disciplines which may be considered expensive and complex (Stokols et al., 1996). 
In addition to the SEM, the medical model of health was also applied to aid interpretation of patients' perceptions of medications and the idea that diabetes could be cured as they perceived that medications should be sufficient alone to maintain good glycaemic control levels (chapter five). Additionally, the medical model aided understanding of how most of the HCWs in this study followed the medical model, and they were task-focused by focusing mainly on the prescribed medications without considering other needs of the patients such as exploring herbal usage (chapter six). Also, the medical model aided understanding of challenges related to the health care system and the impact of health insurance policies on patients' management of diabetes (chapter seven). The medical model of health care is presented next.

\subsubsection{Medical model of health care}

The main features of the medical model are a focus on physical symptoms of an individual, cause-effect approach, adherence to guidelines of treatment and power is mainly held by the health care providers (Shah \& Mountain, 2007). Furthermore, in the medical model, an individual's health is limited to the physical or biological component without taking into account other essential social, spiritual and psychological determinants of an individual's health (Shah \& Mountain, 2007; Wills, 2014). Patients are labelled as sick bodies instead of whole individuals (Giddens \& Griffiths, 2006) which is inconsistent with the essential components of a patient's health; physical, psychological and social aspects as proposed by the WHO (WHO, 1948).

The medical model is referred to health as basically the absence of disease or illness (Naidoo\& Wills, 2016). Individuals are described as machines in the medical model when there is malfunction or defect in any part of the human body, and this defect has to be fixed and returned to the pre-defect status (Pearson, Vaughan, \& Fitzgerald, 2005). The medical model perceives the human body as disconnected parts that have to be recovered or studied separately. This notion was introduced early to studying the human body, and it is known as the reductionist approach (Naidoo\& Wills, 2009). In the reductionist approach, the human body is broken down into smaller parts from the body to systems to organs to tissues to cells (Pearson et al., 2005). The needs of each part have to be fulfilled by a specialist who has extra training or skills in this affected part which is considered the main limitation of the medical model. The medical model lacks a holistic view as it does not perceive the individual beyond the physical dimension and underplays the family, cultural and social influences on an individual's health. The risk factors for chronic conditions such as Type-2 diabetes are related to lifestyle factors which are 
primarily social factors (Naidoo \& Wills, 2009). This separates an individual from his or her social and cultural factors which, in reality, have either a positive or negative impact on the individual's health (Shah \& Mountain, 2007). The concept of 'holism' is well integrated within systems theory (Bertalanffy V, 1968) in opposition to the reductionist approach in the medical model of health. In adopting a holistic approach, the researcher has to view the phenomenon entirely as a whole system rather than a set of parts (Mele, Pels, \& Polese, 2010). The whole system interacts with the external environment and it is an open system. The system's parts affect each other, and any defect in one part has consequences on other parts (Pearson et al., 2005).

Treatment and cure of the patient are the main targets of the medical model with less regard to health promotion and disease prevention (Wills, 2014). However, a cure is currently impossible in chronic conditions such as diabetes which is one of the limitations of this model (Giddens \& Griffiths, 2006). Within the medical model, the HCWs' knowledge is viewed as being credible and trustworthy and provides the sole accurate source of health information (Giddens $\&$ Griffiths, 2006). Further, perceptions and beliefs of patients or family members are less appreciated by health care professionals (Shah \& Mountain, 2007). As others have reported, the family members and friends with diabetes were considered as good sources of information (Al-Maskari et al., 2013; Bakkar et al., 2017). Routinisation in nursing care and depersonalisation of patients are examples of the impact of the medical model on nursing practice (Pearson et al., 2005). Routinisation in nursing care refers to care which is standardised to all patients with a certain disease without focusing on the patient as an individual (Pearson et al., 2005). Therefore, providing routine nursing care without recognition of personal preferences and treating the disease process rather than the individual.

In the depersonalisation of patients which is often a feature of the medical model, the patient is labelled with the disease and as a sick body rather than known as a person (Giddens \& Griffiths, 2006). For example, during my review of research studies about diabetes in Jordan the majority of them referred to the study participants as diabetic patients or diabetic subjects or study subjects (Ajlouni et al., 2008; Al Omari et al., 2009; Habashneh et al., 2010; Khattab et al., 2010; Abu- Qamar \& Wilson, 2011; Al-Amer et al., 2011; Wazaify et al., 2011; AlKhawaldeh et al., 2012; Al-Sarihin et al., 2012; Adwan \& Najjar, 2013; Awwad et al., 2015; Irshaid, 2014). The reasons behind that are: the medical model is the dominant model in Jordan; there persists a strong influence of the medical model on nursing practice in Jordan; most of the studies are conducted by physicians and limited roles of nurses in research (Zahran, 2011). 
Lack of time and lack of practical research skills are the reasons behind nurses not taking part in research projects (Kenkre, 1997).

Nurses in Jordan base their approach to care primarily on the medical model. The dominant method of delivering nursing care is task-oriented, and the focus on patient-centred care is also limited in Jordan (Zahran, 2011). Additionally, the functional nursing approach or task focused care is a common method of nursing care organisation in the public sector in Jordan which may result in a fragmentation of patient's care and limited holistic care of patients (Shuriquie, While, \& Fitzpatrick, 2008). The functional models of nursing care reflect a view of nursing as a broad set of divided functions that can be carried out by multiple nurses (Dubois et al., 2013). This also explains how the medical model shapes nursing practice in Jordan.

Development of nursing in Jordan was influenced by the medical profession and the American model of nursing regarding curriculum and structure (Shuriquie et al., 2008; Zahran, 2011). Therefore, there might be a gap between theory and practice as the majority of teaching books are based on the American context which may be inappropriate or not transferable to the Jordanian context (Zahran, 2011).

Treatment in the medical model depends mainly on medications which are considered as one of the main features of the medical model (Giddens \& Griffiths, 2006). Visible psychomotor care such as administering medications or measuring blood pressure is valued more than psychological and social health care needs of patients (Pearson et al., 2005). Nurses are more concerned to complete visible tasks (priorities) as a must before finishing their duty. Moreover, accountability is based on completion of physical care needs of the patients (Pearson et al., 2005).

The health belief model (HBM) (Rosenstock, 1974) aided me in an understanding of the findings related to patients' perceptions of diabetes complications and how they perceived the threat of diabetes complications (chapter five). The HBM is discussed now.

\subsubsection{Health Belief Model (HBM)}

The HBM is a conceptual framework that has been used to identify the role of beliefs in health decisions and to understand the reasons behind people's inability to take part in disease prevention programmes, and health promotion initiatives (Rosenstock, 1974; Becker, Maiman, Kirscht, Haefner, \& Drachman, 1978). The HBM was proposed by Rosenstock (1966) then modified by Becker in 1974 and was used to predict health prevention activities such as 
screening and vaccination uptake (Naidoo \& Wills, 2009). In the HBM, patients with a high perceived threat are more likely to engage in healthy activities and change their behaviours (Becker et al., 1978).

The HBM is a conceptual framework that discusses the individual's perception of risk and health beliefs within six components (Becker et al., 1978). These are: the likelihood of experiencing illness (perceived susceptibility); whether engaging in a new preventive behaviour will be beneficial (perceived benefits); beliefs about the barriers that may hinder adapting a new behaviour (perceived barriers); beliefs about the consequences of developing illness (perceived severity); non-modifying factors such as age and gender affects individual's beliefs about health behaviour; and factors such as incentives and health education campaigns that stimulate and trigger the patients to take action and change their habits. These latter elements are known as cues to action and self-efficacy was added in 1988 to the HBM (Rosenstock, Strecher, \& Becker, 1988).

The HBM has been previously applied to aid understanding of diabetes management in the Middle East countries; Iran, Oman, Saudi Arabia and Palestine. Three Iranian studies were found using the HBM to explore the effectiveness of educational interventions based on the HBM on promoting diabetes management behaviours among patients with Type-2 diabetes (Bayat et al., 2013; Dehghani-Tafti et al., 2015; Shabibi et al., 2017). Another Saudi study showed the effectiveness of using the HBM in assessing knowledge and attitudes of Type-2 diabetes among patients with diabetes (Al-Mutairi, Bawazir, Ahmed, \& Jradi, 2015). Whilst another study conducted in East Jerusalem identified factors associated with diabetes management behaviours amongst Palestinians with Type-2 diabetes and examined the contribution of health beliefs (HBM constructs) (Daoud, Osman, Hart, Berry, \& Adler, 2015).

The HBM model has been utilised as a theoretical framework by Subhi, Kendall, Al-Shafaee, and Al-Adawi (2016) to identify the health beliefs associated with diabetes self-management among 29 Omani patients with Type-2 diabetes. The method of data collection was a structured, open-ended interview. This study's findings revealed six main barriers affecting diabetes management behaviours among patients: the cost of blood glucose self-monitoring, negative impact on social interactions, negative feelings associated with living with diabetes, lack of accurate practical and culturally-sensitive health education about diet and physical activity, fatalistic attitudes and low self-efficacy. 
Although the HBM has been widely used to explain individuals' health behaviours, it has received several criticisms (Naidoo \& Wills, 2016). The HBM assumes that different kinds of internal and external triggers/incentives must occur to stimulate an individual's behaviour change. However, individuals may take action and change their behaviours without triggers, or their behaviour change may not respond to the triggers. The HBM assumes that health behaviours are under an individual's control and determined by personal beliefs. However, my findings indicated that other factors might affect patients' illness and some of these were beyond patients' control such as sociocultural factors and health policies. A further critique of the HBM it that it assumes that individuals are rational decision makers yet decisions are influenced by many issues such as emotional status (Taylor et al., 2006).

In addition to the HBM, the locus of control (Rotter, 1966) theory was used to aid understanding of the attitudes, knowledge and perceptions of patients regarding diabetes at the time of diagnosis, their reasons as to why they thought they had developed diabetes and the stressful life events that preceded their diagnosis with diabetes (chapter five). Also, many patients in this study were not able to overcome the impact of the external circumstances on their health. As will be shown in chapter five, patients used fatalistic statements related to religion and especially Allah which indicated that these played an essential role in the patients' perceptions and management of diabetes. Therefore, Rotter's locus of control was drawn upon to explain the complexity of these perceptions and beliefs factors which had an influence on the glycaemic control of patients with Type-2 diabetes in my study. The locus of control theory is discussed now.

\subsubsection{Locus of Control (LOC)}

Social learning theory (Rotter, 1954) provided the general theoretical background for the conception of the nature and effects of individuals' external and internal control of reinforcement. Based on social learning theory, Rotter (1966) reported that patients' locus of control indicated to what extent they feel that they have control over the event or whether the event is perceived as being outside their control such as a result of luck, coincidence, fate or chance. Individuals who display strong beliefs in an external locus of control (LOC) do not see themselves as having any responsibility for their illness. Therefore, individuals with an external LOC believe that the outcomes of events are beyond their control and they attribute the development of illness to external circumstances (Rotter, 1966). On the other hand, individuals with an internal LOC recognise that they should take responsibility for their own health and 
maintain a balance between physical, psychological and spiritual aspects without blaming external factors (Rotter, 1966). In this respect, Individuals with an internal LOC are considered as being proactive by showing more problem solving and coping skills than those with external LOC who feel no responsibility to solve the problem (Besen, Günüşen, Sürücü, \& Koşar, 2016).

The LOC theory has been utilised as a theoretical framework in many studies in Turkey and USA related to diabetes to explain the likelihood of adherence of people to a diabetes management plan (O’Hea et al., 2005; Besen et al., 2016). Individuals with internal LOC are more likely to follow the recommended diabetes management behaviours (DMBs) because they believe that fate or chance will not perform DMBs for them (O'Hea et al., 2005; Besen et al., 2016). O'Hea et al. (2005) also reported that individuals with external LOC had poorer HbA1c levels than those with an internal LOC.

\subsection{Summary}

This chapter has provided the rationale for choosing a research methodology to address the aim and objectives of the study. A qualitative approach was the most appropriate choice for this study to gain an in-depth understanding of factors associated with poorly controlled Type-2 diabetes in Jordan. An ethnographic design was used in this study to understand shared and common experiences among patients with poor glycaemic control levels in their social, cultural and economic contexts. Multiple methods of data collection (focus group, semi-structured interviews, participant observation and documentary review) were used to explore the factors associated with poorly controlled Type- 2 diabetes to achieve a comprehensive understanding of the factors involved.

This chapter has provided detailed information about the inclusion and exclusion criteria for the selection of participants, the recruitment process, methods of data collection, the roles of the researcher in participant observation, data handling and analysis and ethical considerations. In this study, Lincoln and Guba's evaluation criteria (1985) together with Hammersley's evaluation criteria (1992) were used to evaluate my study findings. I have also adopted a reflexive approach in all stages of my study and justified my reason for doing so.

Additionally, this chapter has discussed the main theories that are drawn from health promotion, psychology, systems theory, social learning theory, the socio-ecological model and 
the medical model of health care which have been applied to aid understanding of my findings. A number of theories were drawn upon to explain the complexity of factors which influenced the glycaemic control of patients with Type-2 diabetes. Critiques and limitations of these theories have also been discussed. This explanation of the theories prior to the presentation of the findings and discussion chapters is to enable the reader to make sense of the findings and discussion chapters which follow. It should be emphasised that the relevance of these theories only became apparent from analysing the data generated.

The following chapter includes an introduction to the study's findings and development of the key themes in this study. 


\section{CHAPTER FOUR}

\section{INTRODUCTION TO THE FINDINGS}

\subsection{Introduction}

This chapter includes information about the characteristics of the participants in this study and the main themes which emerged from analysing the data. There were 38 patients with poorly controlled T2DM in this study, of whom nine managed their condition by oral hypoglycaemic tablets and insulin therapy and 23 patients used oral tablets only. Six patients did not complete the section about type of T2DM treatment. The main oral hypoglycaemic tablets that were used by patients were Metformin and Amaryl.

In the following section, the characteristics of the participants who took part in the focus groups and interviews are described.

\subsection{Characteristics of the sample}

This section includes information about sociodemographic data of the participants in this study. First, information about patients with poorly controlled Type- 2 diabetes who participated in the focus group sessions is presented. The second part provides information about health care workers and family carers who took part in this study.

Participants were asked to complete a short sociodemographic questionnaire (Appendix 13) when they attended the focus group session. Sociodemographic data were analysed manually using descriptive statistics including frequencies, mean, median, standard deviation and range (Creswell, 2014). All patients agreed to complete the questionnaires; however, there was missing data in different sections in the completed questionnaires. The sociodemographic data of the patients with Type- 2 diabetes is illustrated in Table 3.

Table 3 illustrates sociodemographic and relevant characteristics of people with Type-2 diabetes $(n=38)$

\begin{tabular}{|l|l|l|l|}
\hline $\begin{array}{l}\text { Demographic } \\
\text { characteristics }\end{array}$ & Frequencies & Percentage (\%) & Missing data \\
\hline Gender & & & \\
\hline Male & 16 & $42.10 \%$ & \\
\hline
\end{tabular}




\begin{tabular}{|c|c|c|c|}
\hline Female & 22 & $57.89 \%$ & \\
\hline \multicolumn{4}{|l|}{ Age (years) } \\
\hline $40-50$ & 13 & $34.21 \%$ & \\
\hline $51-60$ & 12 & $31.57 \%$ & \\
\hline $61-70$ & 9 & $23.68 \%$ & \\
\hline$\geq 71$ years & 2 & $05.26 \%$ & \\
\hline \multicolumn{4}{|l|}{$\begin{array}{lll}\text { Highest level of } \\
\text { education }\end{array}$} \\
\hline No education & 4 & $10.52 \%$ & \\
\hline Primary & 7 & $18.42 \%$ & \\
\hline Secondary school & 12 & $31.57 \%$ & \\
\hline Diploma & 7 & $18.42 \%$ & \\
\hline $\begin{array}{l}\text { University degree or } \\
\text { higher }\end{array}$ & 8 & $21.05 \%$ & \\
\hline $\begin{array}{l}\text { Duration of Type-2 } \\
\text { diabetes (years) }\end{array}$ & & & 5 \\
\hline $1-5$ years & $16 / 33$ & $48.48 \%$ & \\
\hline 6-10 years & $8 / 33$ & $24.24 \%$ & \\
\hline$\geq 10$ years & $9 / 33$ & $27.27 \%$ & \\
\hline $\begin{array}{l}\text { Type of } \text { Type-2 } \\
\text { diabetes treatment }\end{array}$ & & & 6 \\
\hline Diet only & $2 / 32$ & $06.25 \%$ & \\
\hline $\begin{array}{l}\text { Oral hypoglycaemic } \\
\text { tablets }\end{array}$ & $19 / 32$ & $59.37 \%$ & \\
\hline
\end{tabular}




\begin{tabular}{|c|c|c|c|}
\hline $\begin{array}{l}\text { Diet and oral } \\
\text { hypoglycaemic tablets }\end{array}$ & $1 / 32$ & $03.12 \%$ & \\
\hline $\begin{array}{l}\text { Oral hypoglycaemic } \\
\text { tablets }+ \text { herbs }\end{array}$ & $1 / 32$ & $03.12 \%$ & \\
\hline $\begin{array}{l}\text { Insulin and oral } \\
\text { (combined) }\end{array}$ & $9 / 32$ & $28.12 \%$ & \\
\hline $\begin{array}{l}\text { Family history of } \\
\text { diabetes }\end{array}$ & & & 4 \\
\hline Yes & $26 / 34$ & $76.47 \%$ & \\
\hline No & $8 / 34$ & $23.52 \%$ & \\
\hline \multicolumn{4}{|l|}{ Smoking status } \\
\hline Current smoker & 12 & $31.57 \%$ & \\
\hline Past smoker & 4 & $10.52 \%$ & \\
\hline Non-smoker & 18 & $47.36 \%$ & \\
\hline \multicolumn{4}{|l|}{ BMI } \\
\hline Able to calculate & 8 & & \\
\hline $\begin{array}{l}\text { Not able to calculate or } \\
\text { wrong answer }\end{array}$ & 28 & & \\
\hline $\begin{array}{l}\text { Answering without } \\
\text { writing height and } \\
\text { weight }\end{array}$ & 2 & & \\
\hline $\begin{array}{l}\text { Body Mass Index ( } \\
\text { BMI) }\end{array}$ & & & 10 \\
\hline Normal & $16 / 28$ & $57.14 \%$ & \\
\hline
\end{tabular}




\begin{tabular}{|c|c|c|c|}
\hline Over weight & $8 / 28$ & $28.57 \%$ & \\
\hline Obese & $1 / 28$ & $03.57 \%$ & \\
\hline Do not Know & $3 / 28$ & $10.71 \%$ & \\
\hline Marital status & & & 2 \\
\hline Single & $2 / 36$ & $05.55 \%$ & \\
\hline Married & $31 / 36$ & $86.11 \%$ & \\
\hline Divorced & $1 / 36$ & $02.77 \%$ & \\
\hline Widowed & $2 / 36$ & $05.55 \%$ & \\
\hline $\begin{array}{l}\text { Monthly income in } \\
\text { Jordanian Dinar (JD) }\end{array}$ & & & 10 \\
\hline$\leq 299$ JD & $2 / 28$ & $07.14 \%$ & \\
\hline 300-500 JD & $20 / 28$ & $71.42 \%$ & \\
\hline $501-1000 \mathrm{JD}$ & $5 / 28$ & $17.85 \%$ & \\
\hline$\geq 1000 \mathrm{JD}$ & $1 / 28$ & $03.57 \%$ & \\
\hline $\begin{array}{l}\text { Attendance at formal } \\
\text { diabetes education } \\
\text { programme in the last } \\
\text { year }\end{array}$ & & & 3 \\
\hline No & $21 / 35$ & $60 \%$ & \\
\hline Yes & $14 / 35$ & $40 \%$ & \\
\hline
\end{tabular}

All focus groups were conducted in the comprehensive health care centre. Eight FGs $(n=38)$ were conducted with a mix of males $(n=16)$ and females $(n=22)$ with Type- 2 diabetes. The number of participants per focus group ranged from 3-6, the average duration was 70 minutes. I also interviewed 10 (HCWs); three nurses, a physician, dietitian, pharmacist, lab technician, dentist, receptionist and supervisor of Health Promotion. Six patients' family carers with 
different caring relationships were also interviewed; two sons and four spouses. The average duration of interviews was 30 minutes.

Having discussed the characteristics of the participants in this study, the main themes derived from the data analysis are presented below.

\subsection{Main Themes of the Study}

There are four main themes which constitute the four findings and discussion chapters. Appendix 20 shows an example of the process of development of one key theme from the initial codes and sub-themes. The first theme which is titled 'Attitudes, knowledge and perceptions of patients regarding Type-2 diabetes' is discussed in chapter five. The socio-cultural influence on patient's health such as the relationship between family carers and friends and their influence on patients' diabetes behaviour management are also considered. The influence of the wider socio-cultural factors is further expanded upon in chapter six which explores the 'Influence of the Jordanian socio-cultural context on patients' diabetes management plans'. The third theme is presented within chapter seven which discusses the 'Challenges related to the comprehensive health care centre and the health care system'. In this chapter, the data related to challenges that hindered management of diabetes at two main levels of care; the health care system level and health care workers (HCWs) in the comprehensive health care centre level are presented and discussed. The final theme titled s 'Facilitators to good glycaemic control levels' (chapter eight) presents the data on patients', carers' and HCWs' suggestions as to what they thought were facilitators to good glycaemic control levels.

\subsection{Summary}

In this chapter, I have presented the characteristics of the participants in this study, and provided an overview of the four main themes which constitute the four findings and discussion chapters.

The following chapter presents and discusses the findings related to attitudes, knowledge and perceptions of patients regarding T2DM. 


\section{CHAPTER FIVE}

\section{ATTITUDES, KNOWLEDGE AND PERCEPTIONS OF PATIENTS REGARDING TYPE-2 DIABETES}

\subsection{Introduction}

This chapter presents and discusses the subthemes and categories related to the attitudes, knowledge and perceptions of patients regarding Type-2 diabetes. In the first subtheme which explores the reasons for developing diabetes, I discuss the reasons for developing diabetes from the patients' perspectives, the fatalistic attitudes of the patients and how the patients attributed the development of their diabetes within the categories of: Whatever happens, Happens, Proactive screening for diabetes, The reaction to classic symptoms of diabetes and Is there emotional diabetes?.

The second subtheme of this chapter titled, 'patients' knowledge of Type-2 diabetes mellitus (T2DM), and its complications' incorporates the categories of Patient's knowledge of diabetes and Patients' perception of diabetes complications. Within these categories, I discuss the findings regarding patients' knowledge of diabetes, their awareness of the risks associated with high blood glucose (BG) levels and their understanding of the impact of poorly controlled diabetes on their lives. The third subtheme presents the data related to patients' perceptions and feelings towards insulin and self-monitoring blood glucose levels (SMBG). The final subtheme of this chapter discusses the main misconceptions identified from participants' accounts about diabetes and its management within two main issues: medications and the seriousness of the condition.

The following subtheme commences with presenting and discussing patients' perceptions of the reasons for developing diabetes.

\subsection{Reasons for developing diabetes from the patients' perspectives}

Many patients and family carers talked about life events that led to the patient's diagnosis or occurred before the diagnosis was confirmed by the doctor. From analysis of the data it became evident that six male patients and three females held fatalistic attitudes which are now presented. 


\subsubsection{Whatever happens, happens}

'Whatever happens, happens' was an expression that a few patients $(n=3)$ mentioned when expressing their fatalistic beliefs and attitudes towards diabetes and its management. Fate was seen as a reason for these patients believing that they did not have control over their own condition. Furthermore, patients used different statements related to fatalism such as, 'God's will', 'inevitable condition' and 'death is once' during FG discussions.

'Death is once', which was translated from the Arabic as (الموت و احد على الرغم من تعدد الاسباب), refers to a few patients believing that they will only die once, regardless of whether they adhere to their management plan or not. Even though the causes of death are several, a person dies only once. These patients perceived death as a part of their fate which was predetermined by God. Therefore, they did not acknowledge that adherence to a management plan was important. They also felt hopelessness as they had no expectation of future improvement of their condition.

Patients mainly used these fatalistic expressions when they were asked to talk about the reasons for developing diabetes or their difficulties in adhering to their treatment plan. A few patients expressed fatalistic attitudes when they were asked to talk about the causes of diabetes:

I got diabetes because of psychological distress. The psychological distress which my son gave me a hard time because he didn't want to study and I was so stressed. I kept following my son's study, and I was always stressed and family history too. I was a diabetes carrier, and it happened because of the psychological thing. Anything you are a carrier with will appear (Female patient 3. FG 4).

Diabetes is forced on us regardless of our efforts so we should accept it (Male patient 6. FG 4).

Diabetes was perceived as an inevitable condition especially among patients who had a positive family history and went through stressful life events prior to diagnosis. The notion of being a carrier for diabetes is also a misconception. Patients' perceived lack of control over external risk factors for diabetes underpinned their fatalistic attitudes. For example, family history and stress were perceived by these patients as unavoidable causes that increased their chances of developing diabetes and were beyond their control. Furthermore, these patients perceived that acquiring diabetes was eventually caused by external forces over which they had no personal control. In their sense, they were not to be blamed for developing diabetes as it was caused by 
factors outside of their control. The patients cited in the above quotes predicted their diagnosis of diabetes was unavoidable because of family history and stress. Therefore, these patients believed that they did not have any responsibility for developing diabetes because of the overwhelming influence of external factors. These patients also believed that the external forces were more influential than their personal agency in the development of diabetes.

Rotter (1966) reported that patients' locus of control indicated the extent to which they feel that they have control over the event or the event is outside their control and attributable to luck, fate and chance. These patients displayed strong beliefs in an external locus of control regarding their diagnosis of diabetes. In any behaviour change approach to health promotion, these patients could be seen to be contributing to developing diabetes because they did not look after themselves especially with respect to maintaining a healthy lifestyle (Naidoo \& Wills, 2016). Although most Arabs strongly believe in fate and destiny due to the religious faith, they are also encouraged to believe in self-responsibility. Responsibility towards one's health is emphasised in the Qur'an and the Islamic Hadith (Nabolsi \& Carson, 2011).

Difficulties in adhering to the diabetes management behaviour plan induced six patients out of 38 patients to express feelings of resignation, hopelessness and fatalistic beliefs to justify their difficulties:

Regarding physical activity, I don't walk. I don't follow the proper diet too. So these are the reasons why my blood glucose is so high. I love sweets, and sometimes you can't control yourself in eating. The person may reach the hopeless stage, and the death is once, and I don't know why I think like this. I know this thought is wrong and diabetes affects everything, eyes and other things (Female patient 4. FG 3).

This female patient's fatalistic attitudes appear to be associated with her poor adherence to diet, medications and physical activity. She also felt hopeless which influenced her behaviour. She gave examples of the lifestyle factors that were responsible for her high BG levels, but at the same time, she used fatalistic attitudes to express her feelings of difficulties in adhering to her diabetes management plan. Although this female patient believed that she is partially responsible for her high BG levels, she attributes blame on the fate that was beyond her control. The overwhelming power of fate was perceived by these patients to justify their difficulties in controlling their BG levels. She also acknowledged that her negative attitudes were inappropriate and diabetes complications may develop as a result of that. 'Hopelessness' was mentioned which is viewed as an indicator of fatalism and associated with poor adherence, 
decreased quality of life and poor BG levels (Egede \& Ellis, 2009; Sukkarieh-Haraty, Egede, Kharma, \& Bassil, 2017). The patients' expressions of fatalism were consistent with the three main dimensions of diabetes fatalism: emotional distress, hopelessness and powerlessness (Egede \& Ellis, 2009). Later in the discussion, another female patient replied to my question about doing self-testing at home:

I gave up testing for a year, and I did not buy strips. Now when I get up, I have shakiness, and when I test my blood glucose, I find it 150 but not every time I test. I stopped buying things for diabetes. I also got bored and as this sister said 'the death is once, whatever happens, happens' (Female patient 5 .FG 3).

This female patient (40-50 years old) who has been diagnosed with diabetes less than five years also supported other patients' fatalistic attitudes towards diabetes management behaviours. This patient's responses showed that she had become disinterested in the long-term management of diabetes which reinforced the feeling of fatalism and hopeless. This patient's growing disenchantment with self-managing her condition increased her tendency towards fatalism and learned helplessness rather than trying to overcome any barriers. This patient also felt comfortable in stopping self-testing, and she found fate as an 'escape' or an excuse for not being able to manage her condition. This patient's responses also indicated there was a link between fatalism and reduced perceived stress or 'stress relief'. She used fatalistic expressions to avoid stress rather than worrying about the outcomes of poor adherence. Also, by stopping the testing, she was in a state of denial as each test may just have highlighted her difficulties in being able to adhere to the management plan, so by not testing she could remain in ignorant bliss and this to some extent reduced her stress.

A male patient used religious expressions to shift the responsibility for his own condition to God or Allah in Arabic, and that diabetes was from God:

I try to walk in the morning, noon and evening. I walk when the weather is so hot. I walk as much as I can. As I told you according to the instructions that I received from the centre I try to apply them because if I don't apply them, I will stay with the same disease. I want to beat the disease and thank, Allah it is Allah's willing. When I am a patient with diabetes, it is from Allah (Male patient 5. FG 8).

This patient gave examples of different positive behaviours such as doing regular physical activity and following the doctor's instructions in order to minimise the risk of developing complications. However, he also emphasised the role of religious beliefs and faith in 
controlling his condition and attributed developing diabetes to a higher power 'Allah' as a sign of coping and acceptance of his condition. While these coping strategies involve Allah as the most influential external power, he believed that Allah's control reduced his own worry. He also pointed out that all life circumstances were controlled and predetermined by Allah, but at the same time, he acknowledged that he should take responsibility for his condition. This implied this patient believed he had little or no personal control over his fate.

Similarly, a cross-sectional study aimed to explore how perceptions of self-efficacy, health locus of control and outcome expectancy affected adherence of 30 patients with Type-2 diabetes in Saudi Arabia (Albargawi et al., 2016). Most of the participants acknowledged the importance of visiting the doctor and following medical instructions, but also they believed that God was responsible for whether their condition got better or worse (Albargawi et al., 2016). Many of the Qur'an's phrases and Islamic beliefs encourage patients to take responsibility for their own health and maintain a balance between physical, psychological and spiritual aspects which is consistent with the concept of wholeness and internal locus of control (Nabolsi \& Carson, 2011; Albargawi et al., 2016). A phenomenological study explored perceptions of 19 Jordanians with heart disease regarding the meaning of spiritually. This study's results showed that most of the participants viewed illness as a 'will of God' and shared the same belief that is 'whatever happens, must happen' (Nabolsi \& Carson, 2011). For Muslims, most of the life events including developing illness and recovery are explained regarding 'God's will' (Jeragh-Alhaddad, Waheedi, Barber, \& Brock, 2015).

Two family carers also talked about the fatalistic attitudes of their relative as a reason for a high BG level:

The negative thoughts about death, which all of us will die eventually, so control our diet or lifestyle are unnecessary (Son. Interview 2).

My husband says 'the age is one and God is one'. He doesn't care even for medications. I try to put the medications in front of him, but he does not want to take them (Spouse. Interview 5).

Fatalism acted as an obstacle to these patients achieving good BG levels. The son and the spouse in the above quotes believed their life expectancy was pre-determined by God and therefore whatever they did they would not live any longer. From their perspectives, all life events were predetermined and fatalism would defeat patients' attempts to adhere to diabetes management behaviours. This spouse associated poor adherence to medication with her 
husband's fatalistic beliefs. These patients with an external locus of control believed that adherence to a treatment plan was not essential because God controls their destinies. These patients also perceived death and illness as a part of their fate; therefore, they did not acknowledge that adherence to a management plan was important. Patients' beliefs about death and God are also supported by another study conducted with 20 patients with Type-2 diabetes in Kuwait. Approximately, a third of the patients stopped or adjusted their medication doses due to holding fatalistic beliefs and believing their fate was determined by God rather than therapies (Jeragh- Alhaddad et al., 2015).

Two other patients talked about their lived experience of diabetes concerning the fatalistic attitudes of their family members. These attitudes interfered with patients' dietary adjustment to follow a diabetic diet which they referred to as 'sabotaging attitudes':

My husband tells me: 'eat everything, and it is God's will'. However, I eat reasonably such as I eat one spoon of rice instead of 4 spoons (Female patient 5. FG 3).

People force you to eat by saying God protects you (Male patient 1. FG 5).

In the above quotes, patients reported different perceptions regarding the power of God - as a protector, as someone who determines patients' life expectancy and as someone who gives them a condition. From their perspectives, they felt frustrated towards the fatalistic comments from their friends and families who sought to force them to eat. These patients also felt that their family members hindered their efforts to adhere to a diabetic diet by disapproval and putting the responsibility onto fate. Fatalism was a grounded religious belief among these patients' family members and friends. However, these patients put in more effort to overcome other people's attitudes by adhering to a diabetic diet. Patients' family carers reported that patients should not indulge in any behaviours against the will of God which discouraged them from taking more responsibility in their diabetes management. Therefore, patients' family carers did not believe that patients had a high chance of developing diabetes complications as they thought that Allah would protect their relative.

In Jordanian culture, there are strong rooted cultural and religious beliefs that destiny and fate are determined by 'Allah' (Nabolsi \& Carson, 2011). Similarly, in Saudi's culture, people believe they have control over their own health, but they also believe that God controls all their life events (Albargawi et al., 2016). However, this contradicts many statements in the Holy Qur'an which emphasises treatment and disease prevention (Nabolsi \& Carson, 2011). 
A belief in fatalism added an extra burden and another risk factor for developing diabetes and high BG levels in my sample. The above patients' accounts showed that these patients $(n=8)$ felt they had limited personal agency to prevent the development of diabetes or control their BG levels. Two-thirds of patients $(n=6)$ who expressed fatalistic beliefs, were more likely to have a lower education level (secondary), be of a younger age (40-50 years) and have a shorter duration of having diabetes (1-5 years). These patients were of a younger age and had diabetes for a shorter period so their risk of developing complications was high. Fatalism was linked with religion and the power of Allah, and was understood through applying the locus of control in my study. Fatalistic statements related to religion and Allah indicated that these played an important role in patients' perceptions and management of diabetes. For those patients with strong religious beliefs, their religion shaped and informed their health behaviours. They believed that their condition could not be changed or modified despite their attempts to achieve treatment goals. They also believed that there are external forces due to the fate that exist (out there) that defeated their personal efforts to adhere to their management plan. They justified drawing on external forces to avoid self-blame and feelings of guilt for their difficulties in achieving good BG levels. Therefore, fate was used either as a stress relief, acceptance or explanation of their inability to follow their treatment plan. Two patients believed that fatalism helped them to cope and accept their condition. These fatalistic beliefs resulted in a lack of motivation to change behaviour and poor adherence to treatment plans. This external power was beyond patients' control, so it was used as a logical explanation of the causes of their diabetes and their high BG levels.

Family history is also beyond patients' control, and stressful events may also be, but these were reported as factors for looking after their health as they perceived that whatever they did they would still develop diabetes in the future. These patients did not understand how they could prevent or delay the onset of the condition. For a fifth of patients $(n=5)$ with a family history out of 26 patients with a family history of diabetes, these were diagnosed after proactive screening for diabetes which is presented now.

\subsubsection{Proactive screening for diabetes}

Family history was reported as the primary risk factor associated with the development of diabetes among two-thirds of the patients $(n=26)$ in this study. However, proactive screening for diabetes was reported by $5(20 \%)$ of the 26 patients with positive family history: 
My mother has diabetes and her sisters too. So when I was visiting my friend, I tested my blood glucose and I didn't have my breakfast yet, and the test was high 130 so directly I said it is family history that has a role. Then I did fasting blood sugar (FBS), and it was high too so then I have started treatment for diabetes (Female patient 1. FG 7).

This patient was aware that she was at high risk of developing diabetes due to her family history. This perception encouraged and motivated her to do self-screening for diabetes by measuring her FBS. She clearly attributed diabetes to familial reasons. Up to two-thirds $(n=26)$ of patients in this study had a family history of first degree relatives, mainly their parents, with the disease which added another risk factor for developing diabetes among them. Family history was the most common risk factor reported by patients in this study. Similarly, a study of assessment knowledge among 180 females with diabetes in Jordan, reported that $126(70 \%)$ had a family history of diabetes as a first-degree family member (El-Qudah, 2016). Type-2 diabetes has a strong association with a positive family history (ADA, 2017).

Many patients supported the female patient's view in the previous quote that a family history of diabetes increased their susceptibility to developing diabetes:

My blood glucose concentration was 420 then I was sent to see the diabetic doctor. It was family history from my father and mother sides. I am worried about my children and grandsons because of diabetes in the family from the youngest to the oldest one. It is good I got diabetes while I was old. I am 53 years old (Female patient 3 (p1). FG 6).

I kept following my son's study, and I was always stressed and family history too. I was a diabetes carrier, and it happened (Female patient 3. FG 4).

Patient (1) felt worried that her family members might develop diabetes because of the positive family history. These patients talked about how awareness of the family history increased their susceptibility to diabetes. However, the notion of being a carrier for diabetes due to family history is a misconception as previously reported.

The findings from this study showed how the previous formal educational programmes in the health centre provided a good example of a proactive screening programme as patients were motivated to seek health education:

I came with my sister to attend the lecture here, and I tested my fasting blood glucose, and it was $167 \mathrm{mg} / \mathrm{dl}$, and they told me: 'you have diabetes'. So it was for my good that I 
discovered it early. If I stayed till now without discovering it would be worse (Female patient 6. FG 1).

Through my participation in the course, I had my blood sugar tested twice then I discovered I have diabetes (Female patient 1. FG 4).

All participants, patients and family members, who attended the previous educational programme had to be tested and screened for diabetes. In my sample, 11 patients mentioned that they attended the previous educational programmes provided in the health centre. Two patients with positive family history reported that they were diagnosed for the first time with diabetes when they attended the formal educational programmes in the centre. Afterwards, these patients were referred to the family medicine clinic for further investigations. Patients' responses indicated that early recognition of diabetes was praised and supported by them. These patients perceived that any delay in diabetes diagnosis might lead to further complications. A nurse who had been involved in delivering the educational programme talked about her role in screening for diabetes among participants:

We discovered new cases of diabetes during these educational programmes, and their HbAlc readings were high (Nurse. Interview 4).

The above extract showed that the educational programme helped to discover undiagnosed cases of diabetes among people in the community. Also, to increase patients' awareness, the educational programme was utilised to conduct diabetes screening among participants. A fuller discussion of the diabetes educational programme is provided within chapter eight. In the Jordanian literature, a cross-sectional study was conducted to identify the prevalence of diabetes and impaired fasting glycaemia (IFG) with 1121 people aged 25 years and above (Ajlouni et al., 2008). Ajlouni et al. (2008) found that 49 participants had undiagnosed diabetes and 91 had IFG. This means that conducting research in the community helped to discover new cases of diabetes.

Most of the patients in this study reported to the health facility seeking medical help after diabetes symptoms had been detected, and its complications had already developed. The reaction to symptoms of diabetes is discussed now.

\subsubsection{The reaction to classic symptoms of diabetes}

A reactive approach to the symptoms of diabetes was adopted by most of the patients in this study $(\mathrm{n}=19)$ as a response to the signs and symptoms of diabetes. Typical comments included: 
Ten years ago, I ate so many sweets then my eyes became blurred. After that, I went to the clinic, and they told me I have diabetes, and my first fasting blood glucose was 300 (Male patient 1. FG 1).

The first thing was abnormal drinking water, abnormal going to bathroom and pressure in the head radiating to my eye which pushed me to visit the doctor and the blood glucose was 429 (Female Patient 2. FG 8).

Patients reported to the health facility seeking medical help after diabetes had already developed. Patients were not able to cope with the symptoms of diabetes which indicated there was a delay in diabetes diagnosis. As a result of the delay, either the patient progressed to experiencing different complications or had a high BG level at the time of diagnosis. The above patients' accounts showed that they experienced several symptoms of diabetes caused by a high BG level before diagnosis. Early identification of symptoms of diabetes is important to limit any further damage to other body systems (ADA, 2017). Increased thirst and frequent urination were the common shared symptoms of diabetes among patients prior to diagnosis. Approximately half of the patients $(n=19)$ mentioned that they experienced signs and symptoms that guided them to recognise having diabetes before doing blood investigations:

In the beginning, I had to itch, and I was feeling worse with thirst. I was sure, I am a reader, and I read magazines, newspapers and brochures, I was sure that I got diabetes. In the end, I went to the doctor, and she told me: go and do the random blood glucose, and I did it to know what blood glucose was with me (Female patient 3. FG 6).

This patient recognised that extreme thirst and itching indicated early signs of high BG levels. These signs induced her to visit the doctor and screen for diabetes. The patient's responses indicated that she was aware of the symptoms of diabetes that had developed due to the condition. Patients did not report how long they suffered from signs and symptoms before seeking help. A further five patients were diagnosed with diabetes following a routine checkup or visiting the hospital for non-diabetes related reasons:

I don't know what I was feeling in some things such as frequent urination, but I thought of prostate enlargement rather than diabetes. Coincidently, I did regular blood tests because I have hypertension for a long time and my blood glucose was high (Male patient 4. FG 6).

This patient considered that frequent urination and other symptoms were linked to prostate enlargement. Lack of awareness linked to the patient's ignorance of diabetes symptoms resulted 
in a delay in gaining a diabetes diagnosis and taking no action. This patient also had hypertension which might contribute to developing diabetes. Following the routine medical investigations, this patient was told he had diabetes. Four patients were also diagnosed with diabetes during their hospital stays. Examples include:

I was diagnosed when I was an inpatient in The Medical City for having an asthmatic attack. They tested my blood glucose, and it was approximately 500-600 (Male patient 2. FG 1).

I was suffering, and I was hospitalised for a month for the gallbladder operation. I stayed in the hospital for 30 days then they said you've got diabetes. For 17 years I still have suffered from it (Female patient 4. FG 8).

As is apparent, a hospital admission provided opportunistic screening to detect undiagnosed diabetes among inpatients. Patients might have diabetes for many years without obvious signs and symptoms which also explained the unsatisfactory BG levels level upon diagnosis.

Taking other medications which contributed to developing diabetes was mentioned by one patient and her family carer in this study. This patient's son talked about taking corticosteroids which resulted in his mother developing diabetes:

My mother was taking corticosteroids for rheumatism which increased blood sugar and the development of diabetes (Son. Interview 2).

This female patient had a positive family history, and she also lived a sedentary lifestyle. These factors including taking corticosteroids escalated her susceptibility to develop diabetes which is considered an iatrogenic illness. Iatrogenic disease is a complication, or an adverse drug reaction occurred as a result of therapeutic or diagnostic intervention performed on the patient (Permpongkosol, 2011). Draznin (2016) also postulated that high levels of corticosteroids could lead to hyperglycaemia and insulin resistance particularly among people with risk factors such as family history and a sedentary lifestyle.

'Suddenly' was mentioned by many participants in this study to describe how patients were diagnosed with diabetes. Examples included:

Suddenly, we took him to the emergency, and they told us he got diabetes. There were no precursors before (Spouse. Interview 5).

In 2010, I visited the cardiologist, and he told me you have hypertension then I did tests and suddenly I got diabetes (Male patient 5. FG 8). 
I got diabetes suddenly when I was 25 years old. I didn't know about diabetes, I only heard about it (Male patient 2. FG 5).

Patients believed that the onset of diabetes symptoms happened suddenly without experiencing any obvious symptoms. Approximately half of the patients in my study suffered from the symptoms of diabetes before the diagnosis of diabetes was confirmed by the doctor. These patients mentioned that they already had a family history and hypertension which increased their chances of developing diabetes. Patients perceived that diabetes occurred suddenly, but in reality, less than $50 \%$ of B-cell function remains effective at the time of diagnoses of Type2 diabetes (Unger, 2012) which means Type-2 diabetes develops gradually rather than suddenly. Patients were not aware of having diabetes until the symptoms were worsening and their bodies were not able to cope with these symptoms.

Besides experiencing classic signs and symptoms of diabetes, many patients expressed the view that psychological distress caused the onset of diabetes which is explored now.

\subsubsection{Is there emotional diabetes?}

Over a third of patients $(n=13)$ in this study perceived that having psychological distress was the main cause of diabetes. Female patients $(n=9)$ were three times more likely to perceive stress as a cause of diabetes than males $(n=4)$. Many patients talked about the stressful life events that preceded their diagnosis of diabetes. Stress and emotional upset were perceived as a determinant of diabetes among many patients in my study. Patients used different words 'distress', 'shock', 'sadness', 'emotional diabetes' and 'upset' to describe the impact of stress on the development and eventual diagnosis of their diabetes:

All my family members have diabetes, and I got diabetes because of psychological distress (Male patient. FG 2).

I came here, and I was sick then the doctors asked me to do tests, and they told me you have diabetes. I had HbAlc done, and they told me you have emotional diabetes (Female patient 4. FG 8).

Because of sadness. My husband had a heart attack and died then after one year my son who was studying medicine in Egypt had a car accident and died too. He was buried next to his father (Female patient 1. FG 6). 
The above data extracts illustrate that patients believed stress was a cause of diabetes. Patients believed that they developed diabetes due to their grief caused by a family member's death or due to other stressors. These patients were overwhelmed by the influence of stress as a dominant cause of diabetes despite the presence of other risk factors such as family history, obesity and a sedentary lifestyle. Female patient 4 mentioned that the doctors used the term 'emotional diabetes' to refer to the cause of developing diabetes and high blood glucose levels as being attributed to stress, poor emotional health and experiencing negative emotions. Therefore, emotional stress was perceived by a few patients as a reason for developing diabetes, but from my observations, interviews with $\mathrm{HCW}$ s and conversations with doctors the term emotional diabetes was not used. Blood glucose levels may be elevated due to acute physical or emotional stress which is called 'stress hyperglycaemia' (Draznin, 2016). Transient or stress hyperglycaemia occurs when the body responds to stress by releasing hormones such as cortisol and epinephrine that elevates BG levels for a short period (Draznin, 2016). The BG levels usually return back to the normal level after the stress is resolved and has a short time effect.

In my study, patients might already have diabetes before the stressful life event happened. At the same time of the stressful event, the BG levels increased sharply, and the diabetes diagnosis was confirmed among over a third of patients in this study. Therefore, these patients attributed their diabetes diagnosis to stress and external circumstances. Another example of stress as a precursor to diabetes was given by a patient's spouse who referred to events that occurred one year before her husband had a diagnosis:

The main reason for getting diabetes for my husband was sadness. During Ramadan, the sugar increased suddenly because my son was lost so the sugar increased suddenly and he was fasting too. Then he was diagnosed as a patient with diabetes. The HbAlc..... surely, he didn't know he got diabetes because he didn't have a history of diabetes. He had diabetes for one year, and he didn't know. The first reading of HbAlc was $10.5 \%$ very, very high when he started taking metformin (Spouse. Interview 3).

Based on her account, she mentioned that her husband was affected by his son's death. She mentioned that stress was the cause of diabetes for her husband and the high BG level was a sustained physiological reaction to the emotional distress. This patient remained undiagnosed for one year which explained the elevated HbAlc level at the time of diagnosis. These participants' responses reflected an external locus of control by attributing the cause of the 
disease to factors outside their control (Rotter, 1966). Many patients perceived that developing diabetes was unavoidable because of experiencing stress and having a relevant family history:

My parents had diabetes, and I got diabetes because of psychological shock (Male patient 3. FG 2).

My son had an accident and diabetes might be there, but it was not strong. By accident, the blood glucose was high, and the psychological shock might lead to diabetes (Female patient 3. FG 6).

These patients believed that they expected they would have diabetes before the diagnosis was confirmed by the doctor. Patients' responses also showed that they were aware they were at high risk for developing diabetes and their exposure to the external stressful event uncovered and elevated high blood glucose levels. In their experiences, diabetes developed as a bodily reaction to stress. The detection of high BG levels immediately after the family crisis promoted patients' tendency to overestimate the effect of stress. Similarly, a male patient mentioned examples of stressful lifestyle activities which he believed were responsible for developing his diabetes:

I was upset with my manager then I went home. After one or two days, I went to the bathroom many times at night. Then I tested my blood sugar, and the doctor told me you had diabetes and gave me medications (Male patient 1. FG 5).

This patient believed that diabetes was caused by work stress that contributed to developing the symptoms of diabetes eventually.

A female patient narrated her story when first diagnosed with diabetes:

I had shakiness, and they told me it is a result of sadness. I was stressed and sad, and I told my sister, she got diabetes, I am getting cold but she said: no, quickly, because she got diabetes for 10 years, we should test your blood glucose and I told her to take me to the hospital and we found many people there then I told her to take me back to the hotel and test my blood glucose. Because I couldn't stand up, I asked somebody to give me one date, and I thought my blood glucose was low, and I ate half of it because I was afraid of and I had no idea about it then I went back home, and it was 270. I walked for 30 minutes (they say walking is good) and I tested my blood glucose was 270. She told me don't eat anything, and I slept for 4 or 5 hours then I woke up, and I started to be afraid of food and eating a little bit. I told her I got diabetes and I was upset, and they calmed me down when I went to 
the mosque. The next day I was about to faint on the bus on my journey back home, and my family was trying to wake me up by shouting and calling my name. Then my sister in law came and rubbed a date in my mouth, and she was telling me to swallow...swallow then I woke up (Female patient 2. FG 7).

This patient' account showed her ability to narrate the events that happened before and upon diabetes diagnosis. The above example demonstrates a patient's feelings towards diabetes at the time of diagnosis. Lay people's perception of sadness led to stress being perceived as the main cause of diabetes despite experiencing the main symptoms of diabetes. The patient's sister was aware of the symptoms of diabetes; therefore, she recommended doing a BG test. As is apparent, the patient's family member with diabetes was considered as a good source of information.

The above patients' accounts showed that they were not able to overcome the impact of the external circumstances on their health. Many patients with an external locus of control believe that the outcomes of the events are beyond their control and they attribute the development of diabetes to 'stress' and 'coincidence' (Rotter, 1966). In this respect, patients referred to the development of diabetes as 'sudden', and they felt what happened to them was unpredictable and under the control of external powers. As a result, patients identified stress as the logical explanation for developing diabetes despite having other risk factors. Patients were not able to cope with the emerging stressors that led to the development of diabetes and did not take responsibility for their own condition. Patients' inability to control their own lifestyle increased their tendency to blame stressful life events rather than themselves as causes of diabetes.

Family history and stress were perceived by over a third of patients $(n=13)$ as uncontrollable factors and beyond their control. In that sense, patients mentioned that diabetes developed because of having a positive family history and experiencing stressful life events prior to diagnosis. These patients' attitudes were encouraged by lay people's perception, in particular, their family members of the overwhelming influence of stress.

Changing unhealthy behaviours and improved mental well-being was reported by a few patients $(\mathrm{n}=3)$ as facilitators to good BG levels:

Don't be sad, follow the medication regimen and insulin (Male patient 3. FG 2).

Facilitators for diabetes. I will talk about myself. I have to avoid sadness and stress (Female patient 1. FG 8). 
These patients recognised that they should take responsibility for their own health and maintain a balance between physical, psychological and spiritual aspects which is consistent with the internal locus of control (Rotter, 1966). These patients were seeking to develop a more internal locus of control by trying to avoid stress without blaming external factors.

In the previous category, I discussed the reasons for developing diabetes from patients' perspectives. Knowledge of Type- 2 diabetes is one of the important elements in a diabetes management plan. Therefore, this study explored patients' awareness of their condition which is presented and discussed now.

\subsection{Patients' knowledge of Type-2 diabetes and its complications}

All patients were asked questions during the FG discussions to explore their knowledge of Type-2 diabetes and its complications. The following category presents and discusses patients' knowledge of diabetes.

\subsubsection{Patients' knowledge of Type-2 diabetes}

Most of the patients were able to express their knowledge of diabetes as indicated in the following quote which is typical of patients' responses:

There are two types of diabetes, Type-1 and Type-2. Type-1 diabetes is common among children, and Type-2 diabetes occurs with the old age due to bad eating and drinking habits, weakness in the heart muscle and sadness. Sudden sadness may also lead to the development of Type-2 diabetes (Male patient 1. FG 2).

The common perception that age is the discriminating factor between Type- 2 diabetes (older patients) and Type-1 diabetes (younger patients) was mentioned by a few patients. However, the age of patients is no longer a reliable indicator to differentiate between Type- 2 diabetes and Type-1 diabetes (Draznin, 2016). In the above data extract, the patient also talked about many risk factors such as lifestyle factors and psychological crisis that can lead to developing diabetes. He highlighted that stress might cause diabetes. During the FG discussions, I asked the patients about the risk factors for diabetes. Patients' responses were varied, but most of them indicated that family history and stress were the main reasons for the development of diabetes:

Family history such as parents had diabetes is another risk factor for diabetes. Majority of patients may experience psychological shock which increases blood sugar then end up with 
having Type-2 diabetes. The main reasons for diabetes are poor diet and physical inactivity. For instance, people use their cars for travel to the grocery 20 metres away, and all meals contain oil or fried food which increases blood sugar levels then the pancreas gland becomes ineffective. $90 \%$ of the food contains sugar which is not burnt (Male patient 1. FG 2).

Genetic factors too. If there is a family history such as parents with diabetes, it may result in developing diabetes. Severe sadness may lead to diabetes too (Female patient 4. FG 3).

Male patient 1 had previously attended the educational programme about diabetes nine months previously and had retained information and displayed a better knowledge about the causes of diabetes than those who had not attended. A psychological factor was however mentioned spontaneously by most of the patients in all the FG discussions as the main risk factor for diabetes. Patients associated stressful life events with the development of diabetes. Additionally, family history was mentioned by most of the patients as a main cause of diabetes. They believed that positive family history was a strong contributor to developing diabetes among them. A number of patients were also able to mention several factors associated with high BG levels among patients in Jordan. For instance, sedentary lifestyle, physical inactivity, psychological factors and poor diet. Other patients $(n=11)$ talked about the inability of the pancreas to produce enough insulin that enables the body cells to utilise BG. As a result of that, patients may develop diabetes. Examples of patients' responses included:

Defect in insulin secretions in the pancreas which doesn't produce enough insulin to burn the excess sugar in the blood. Indeed, there are signs of frequent urination, thirst, heat sensation in the hands and legs at night besides tiredness and exhaustion (Male patient 4. FG 4).

I know there is an increase in thirst, so you know you got diabetes, dryness in mouth and tongue, quick thirsty and itching. The impairment in blood glucose comes along with damage to the pancreas due to family history from the father or mother side (Female patient 3. FG 6).

Damage in the pancreas was identified by many patients $(n=11)$ as a cause of development of diabetes. Most of the patients were able to mention the main signs and symptoms of diabetes. The patients' awareness could be attributed to over half of the patients experiencing symptoms upon diagnosis, or they observed other family members and friends who also had diabetes. Furthermore, approximately half of the patients had completed a formal education programme 
one year before which increased their awareness of diabetes. For example, there was a dialogue about insulin between two patients who attended the previous education programme in the centre and another patient who had not attended:

Male patient 2: I was injecting insulin in one place which might lead to damage the skin and the body did not absorb insulin effectively.

Male patient 1: The training course gave us background information about diabetes, and they told us do not inject insulin in one place and draw a circle because injecting insulin in one place may lead to damage of skin cells.

Male patient 3: Doctor and dietitian in the educational session told me the instructions on how to inject insulin (FG2).

The above conversation showed different levels of knowledge about diabetes among participants from different educational backgrounds. Despite P2 being a teacher who had been diagnosed with diabetes for more than ten years, he was unaware of the right method of administering insulin. This misperception caused unwanted effects such as lipohypertrophy. Insulin was not also absorbed adequately which placed him at high risk of developing complications. Lipohypertrophy is caused due to injecting insulin many times into the same area in the abdomen (NHS, 2014). Patients $1 \& 3$ who had only completed secondary school showed more awareness regarding rotating injection sites of insulin. The previous dialogue highlighted the health education sessions in the centre provided a group of patients with essential knowledge and skills to manage their condition effectively. The health education sessions also gave these patients the confidence to share their knowledge in a group situation. A few patients $(\mathrm{n}=5)$ talked about symptoms of hypoglycaemia and weight loss. Typical examples included:

Many problems when the blood sugar is low, your legs can't lift you up, and you will experience shivering (Male patient 6. FG 4).

Patient with diabetes loses weight because food doesn't reach to his body, so he feels a headache and pain in extremities (Female patient 3. FG 4).

This male patient described that feeling shaky and shivering were the main symptoms of low BG levels. Weight loss was only mentioned by this female patient as a symptom of diabetes. 
Patients were asked questions to explore their awareness of diabetes complications and how they felt towards diabetes complications and the findings are presented below

\subsubsection{Patients' perception of diabetes complications}

Approximately a third of patients $(n=11)$ in my sample had already developed complications at being diagnosed with diabetes which induced them to learn more about their condition. All patients were able to list a number of examples of diabetes complications. However, knowledge was not enough to change unhealthy behaviours to delay or hinder developing complications among these patients. A third of patients in this study mentioned examples of complications that they already had with a typical response being:

I suffered from complications which diabetes destroyed my retina, kidneys and heart (Male patient 1. FG 3).

Approximately half of the patients $(n=18)$ out of 38 identified that retinopathy and nephropathy were the most common complications of diabetes. This perception was attributable to the high prevalence of kidney and eye problems among patients. Kidney and eye problems were the main complications among seven patients with complications. Oral health problems and hypoglycaemia were only mentioned once by two patients.

A few patients used different phrases such as 'gate for other diseases', 'silent cancer', 'cumulative for all body systems' and 'irreversible damage' to describe how they felt towards diabetes:

I learned that diabetes opens the doors for other diseases/the gate of other diseases in the body such as the poison in the body and it hurts the body so much (Female patient 3. FG 8).

It is a silent cancer (Female patient 1. FG 6).

They perceived that high blood glucose levels cause complications and disturbances in other body systems such as the potentially deadly impact of cancer and poison in the body. These descriptions showed that patients were aware of diabetes complications and reflected their feelings towards the seriousness of diabetes. Patients' responses showed that they perceived the threat of diabetes complications as high, but despite this, they were not making changes to their lifestyle which is inconsistent with the Health Belief Model (HBM). In the HBM, patients with a high perceived threat are more likely to engage in healthy activities and change their behaviours (Becker, 1978). Most patients were also aware that high BG levels caused damage 
to all body systems. Therefore, knowledge alone was not enough to stimulate and trigger the patients to take action and change their habits. These findings are consistent with a crosssectional study aimed to evaluate the awareness of diabetic retinopathy among a sample of 237 patients with Type-2 diabetes in Jordan (Bakkar et al., 2017). Despite the high level of awareness about diabetic retinopathy in the study, the level of patients' compliance with behaviours to reduce the risk of retinopathy was low, with a total of $29.5 \%$ of participants having had an eye examination in the previous year (Bakkar et al., 2017).

Patients' responses indicated that they understood that diabetes influenced all body systems as indicated in the following extracts:

It is linked to ALL body systems (All six patients. FG 4).

All body systems are affected by diabetes such as teeth and gum which is cumulative (Male patient 1. FG 2).

I think high blood glucose levels affect the body in all aspects as this sister said such as the brain and nerves (Male patient 4. FG 6).

Patients' ability to list different examples of complications showed their awareness of the adverse health effects of diabetes on any part of the body. Many patients also experienced signs and symptoms of diabetes upon diagnosis, therefore they were able to recognise the impact of diabetes on their body.

Irreversible damage from diabetes was recognised by four patients in my sample:

Diabetes has complications such as impairment in kidneys and retina. We know diabetesrelated complications when the person becomes sick he is not able to do what he used to do before (Female patient 5. FG 1).

I know if diabetes influences one organ, it is impossible to be repaired and reversed such as eyes and kidneys. So it is important to warn about diabetes (Male patient 3. FG 5).

These patients used the words 'sick' and 'impossible' to describe the irreversible impact of diabetes on the body and its vital organs. Female patient 5 realised that the development of complications limited patients' capability to restore and maintain their life habits, therefore, diabetes had serious effects on patients' lives.

A few patients $(n=3)$ mentioned that witnessing diabetes complications increased their awareness of diabetes complications: 
If the patient is not aware of diabetes complications, he will be aware of due to seeing complications everywhere such as amputation, liver and kidney problems and retinopathy (Male patient 1. FG 2).

Other patients' experiences with complications made patients aware and conscious of the seriousness of diabetes. For example, a male patient was talking about his fear of developing complications:

I am terrified of seeing my uncles' sons who are the same age as me, and some of them lost their legs or have renal failure. Eventually, the patient with diabetes will lose his legs or have the renal failure which forces me to follow the diet too much and decrease the sugar. There is fluctuation in the sugar results. The fear factor affects me (Male patient 4. FG 4).

The above patient's account showed that the experience of witnessing other family members with complications made him feel worried. This extract also illustrated that fear of developing complications acted as a trigger to encourage him to take part in healthy behaviours and healthy dietary habits. With respect to perceived susceptibility, this patient felt that he was susceptible to diabetes complications, therefore, he engaged in activities to lower the chances of developing complications (Glanz, 2002). It is a misbelief that all patients with diabetes will develop complications in the future. The patients can reduce the risk of developing complications if they control their BG levels (ADA, 2017). This patient perceived that high BG is associated with risk of complications which was the reason for his adherence. Witnessing other patients' complications increased his perception of the severity of diabetes that diabetes would lead to the development of serious complications. Perceived susceptibility and seriousness of diabetes motivated a minority of patients $(n=3)$ to consider their condition as being serious which in turn led to a more internal locus of control. This patient's perceptions of the seriousness of diabetes came from his cousins' experience with diabetes complications. In the health belief model, patients with a high perceived threat are more likely to engage in healthy activities and change their behaviours (Becker et al., 1978). A dentist also talked about her personal experience with her father:

Regarding medications, especially tablets, if the patient doesn't know this situation is serious and complications may develop due to uncontrolled blood glucose, the patient will not take tablets. The biggest example is my father's experience. My father didn't consider diabetes is a very dangerous disease until when he got a diabetic foot, then he has started 
to look after himself. We reached the things lately, so the amputation stage occurred (Dentist. Interview 8).

Development of a complication induced her father to adhere to and to develop a more internal locus of control. She witnessed what her father went through and how complications made him aware of the importance of diabetes management. Diabetes complications encouraged her father to change behaviour and follow the treatment plan. In the Health Belief Model, the factors such as complications that stimulate and trigger the patients to take action and change their habits are known as cues to action (Becker et al., 1978).

These patients' beliefs affected their behaviours to engage in healthy activities due to the perceived threat of developing complications. Furthermore, lack of unpleasant symptoms or lack of short-term complications encouraged the dentist's father to believe that diabetes is not serious and did not believe that he would develop complications. The HBM is a conceptual framework that was used to identify and understand the reasons behind people's difficulties to take part in disease prevention programmes (Rosenstock 1974). Although the HBM has been widely used to explain individuals' health behaviours, it has received several criticisms (Naidoo \& Wills, 2016). The HBM assumes that different kinds of internal and external triggers/incentives must occur to stimulate an individual's behaviour change. However, individuals may take action and change their behaviours without triggers, or their behaviour change may not respond to the triggers. In my study, many patients developed complications, but these complications did not stimulate them to change their behaviours or control their BG levels. The HBM does not also perceive the individual beyond the individual level by underplaying the family, cultural and social influences on an individual's health. The HBM assumes that health behaviours are under an individual's control and determined by personal beliefs. In my study, other factors affected patients' responses to their diabetes, and some of these were beyond patients' control such as sociocultural factors and national health policies which are discussed fully in chapters six and seven. In other health models such as the socioecological model, there is no separation between the individual and his or her social context and the relationship between the individual and his or her surrounding environment being reciprocal (McLeroy et al., 1988). Therefore, in order to gain a full understanding of an individual's behaviours, the sociocultural factors and policies have to be taken into account in addition to health beliefs (DiClemente et al., 2009). 
Within this subtheme, data from patients' accounts that showed their knowledge of diabetes and its complications have been presented. Patients' perception of specific diabetes management behaviours including insulin and self-testing are now discussed.

\subsection{Participants' perceptions of insulin and self-monitoring blood glucose (SMBG)}

Nearly a quarter of the 38 patients in my study managed their condition by insulin therapy. However, a few patients expressed their concerns about the use of insulin in their treatment plan. A female patient narrated her social embarrassment with insulin and how her social relationships were affected by taking insulin therapy:

Some of the medications are embarrassing namely insulin needles, and I feel embarrassed. Primarily, we are a socially connected community. When I visit people who invited me, and I should inject myself with insulin I feel embarrassed from injecting insulin. For this reason, I gave up insulin because of the feeling of embarrassment during visiting people. I also have a needle phobia, and Ifeel in pain because I don't inject the needle in the right way, so I am afraid of injecting myself. When I got invited, I did not take insulin because of the feeling of embarrassment, or I missed the right time. For the all preceding reasons, I stopped taking insulin, and I take tablets instead of, but the tablets don't work well because my gland doesn't secrete insulin. I always have high blood sugar and my fasting blood sugar readings always 300 or 370, and this is the normal situation (Female patient 3. FG 4).

The above quote showed the impact of insulin on this patient's social life. Injecting insulin caused her to feel socially awkward; therefore, she stopped taking insulin. She reported that adjusting social habits to cope with the use of insulin injections was difficult. These thoughts made her reluctant to use insulin, and her perception was also compounded by a discouraging attitude held by society. She identified the social factors, and social norms as barriers to adherence to her treatment plan and her embarrassment in social gatherings led to her change to oral medications. She also reported her needle phobia and painful injections that resulted from improper injection technique. This patient's experience may be explained that she perceived social norms and others' perception hindered her to take insulin treatment. In seeking to aid understanding of her experiences, the Theory of Planned Behaviour (TPB) is useful as it places more importance on social habits than the HBM (Naidoo \& Wills, 2016). As was apparent, this patient behaved in response to what other people thought was right complying with 'normative pressure' and subjective norms as the expense of her own physiological health. The Theory of Planned Behaviour (TPB) places more importance on social context, social 
norms, others' perception and social influences (Hill, Fishbein, \& Ajzen, 1977; Ajzen, 1991). Attitudes and subjective norms determine an individual's intention which will lead to behaviour (Huff, Kline, Peterson, \& Green, 2015). An individual's intent to engage in behaviour is a result of perceptions about advantages of engaging in a behaviour, subjective norms with respect to how others would approve or disapprove the behaviour and social pressure to engage or not in the behaviour (Hill et al., 1977; Ajzen, 1991; DiClemente et al., 2009). This female patient evaluated the advantages of taking insulin by considering other people's perceptions of what they would think about her insulin treatment. As is apparent, this patient behaved in a way that other people thought was right as a result of normative pressure. She was concerned about others' attitudes toward her injecting insulin, and she complied with social norms; therefore, her perception of social norms led to behaviour as she felt stigma and fear. Public health campaigns can be utilised for raising public awareness of the needs of people with diabetes, to improve their lives and those at risk of developing diabetes. Her response also showed that she was not aware of the correct technique of insulin injections. She admitted that oral tablets were no longer able to control her BG levels. However, she declined and refused taking insulin because of feeling socially awkward and needle phobia. As a result of that, she had longer periods of high BG levels and her HbA1c was $10.3 \%$ which placed her at high risk for developing diabetes complications in the future. These perceptions were similar to a qualitative study interviewed 21 patients with Type- 2 diabetes in a primary care setting in Malaysia to find out the barriers to use insulin among participants (Hassan et al., 2013). These study findings showed social embarrassment, fear of injections, negative social stigma and inconvenience were barriers to adding insulin to patients' treatment plans (Hassan et al., 2013).

Poorly controlled diabetes was also attributed to a lack of patient's awareness of insulin therapy as mentioned by a few HCWs $(n=3)$. A response which is typical of all three HCWs was:

Unfortunately, the patient takes insulin for one year, and his blood sugar remains uncontrolled, and we discover later this patient stores insulin wrongly, or he injects himself in the wrong area (Nurse. Interview 7).

This nurse explained that patients were not aware of the correct way of storing and taking insulin. Taking insulin in an inappropriate way or incorrect storage limited the effectiveness of insulin and led to high BG levels among 2 out of 9 patients with insulin therapy in this study. The formal education sessions in the centre had previously provided patients who attended with 
essential skills and knowledge related to administering and storing insulin. Two HCWs also reported patients' fear of initiating insulin therapy:

When I add insulin based on the guidelines to the treatment plan, most of the patients are afraid of having insulin as end-stage, so I try to educate them. The patient may be worried regarding injections, and he may have a phobia of needles or hypoglycaemia (Physician. Interview 1).

People feel terrified when they are switched to take injections. People are afraid of injections. Many patients don't like to be switched from tablets to insulin (Dentist. Interview 8).

From the HCW's accounts, patients considered insulin as a 'last resort' when all other types of treatment were no longer effective. Therefore, patients associated insulin therapy with a serious stage of diabetes and the development of complications. These patients' perceptions of insulin are supported by a quantitative study conducted with 223 patients in Jordan (Al-Khawaldeh et al., 2012). This study's findings found that insulin was perceived by patients as an indicator of poor glycaemic control. This perception was attributed to more than half of the patients who used insulin who had their $\mathrm{HbA1c}$ greater than 7 and insulin was added as a result of high BG levels among patients. Patients misunderstood the progressive nature of diabetes, that they required the use of insulin therapy when non-insulin treatment options are no longer able to control blood glucose levels (IDF, 2017).

Most of the HCWs in my study were aware of the patients' concerns about insulin initiation by trying to reduce any negative patients' feelings towards insulin. Fear of painful injections and hypoglycaemia were the main barriers reported among the patients. Fear of injections was also associated with patients' resistance to accepting insulin to be added to their treatment programme:

Because I don't want the doctor to add insulin to my medication regimen, I do not tell her about the increase in my blood glucose levels. I only take tablets because it is hard to inject myself. No (Female patient 4. FG 3).

Verbally, this female patient provided the doctor with inaccurate results of BG levels. Several doctors routinely asked patients to bring their own laboratory results to be interpreted and written in patients' records. I observed that sometimes patients did the laboratory tests in another health facility and did not bring the results with them during their visit to the centre. 
Therefore, the doctors might change the patient's management plan based on results provided verbally by the patient. She expressed her feelings about painful injections as a barrier to insulin treatment. Therefore, she preferred oral medications over insulin injections for her convenience and fear of needles. These feelings resulted in a delay in starting insulin therapy, leading to longer periods of high BG levels. However, a male patient had a positive attitude towards insulin therapy:

I asked to take Lantus for decreasing of other medications. However, it affects the person when he injects himself. However, insulin is better than other medications because I have problems with my kidneys. So to relieve the load on kidneys, I asked to take insulin (Male patient 2. FG 3).

This patient was aware that insulin is needed to enhance other body organ functions such as the kidneys. This patient indicated that he was willing to initiate insulin and taking insulin was more effective than other treatments. This patient's intention to take insulin was associated with his positive personal beliefs about the long-term benefits of insulin. This male patient, a university graduate, (aged 61-70 years old) was one of the most educated patients in this study who had also attended the previous education programme provided in the comprehensive health care centre. His responses during the discussion showed that he was aware of many aspects of diabetes management. None of the other patients who used insulin reported similar feelings expressed by this patient.

Many patients in this study did not test their BG levels at home despite a quarter of them who were taking insulin. A few female patients reported that they avoided glucose testing due to the emotional anxiety testing caused them. None of the male patients reported this feeling of anxiety associated with glucose testing. Other reasons than stress such as the cost of the strips and needle phobia will be discussed in chapter seven. Fear of self-testing was linked to stress and avoidance behaviour among a few female patients. The following quotes illustrate this point:

Self- monitoring makes obsession which every minute you bring the glucometer, so I prefer to stay on the monthly test (Female patient 5. FG 1).

From the things that make the patient unhappy the excess doing self-monitoring tests which when you eat the sugar is low while during fasting the sugar is high. These readings make you stressed. Self-monitoring makes patients psychologically stressed then sugar increases (Female patient 3. FG 4). 
These female patients associated frequent BG testing with an 'obsession' that elevated their BG levels. These thoughts made these female patients avoid testing their BG at home; therefore, they only relied on the tests done at the health centre. They were scared of the daily fluctuations of the BG values which caused them stress especially when BG levels were high. These female patients felt satisfied with avoiding self-testing, and this relieved their stress, and therefore they relied on monthly testing to monitor their BG levels. Therefore, the psychological factor was seen as a barrier to do SMBG.

Most of the patients waited to test their BG until either they felt symptoms of diabetes or they did not feel well (reactive approach). Typical responses included:

If I see myself being tired due to increase or decrease in blood glucose, immediately I test my blood glucose, so the glucometer is so good if it is available. Now I don't test my blood glucose (Male patient 2. FG 5).

When I see myself being tired. I may test 3 or 4 times a day, and I feel stable I test 1 or 2 and some days I don't test so it depends (Female patient 1. FG 7).

These patients tested their BG when they experienced obvious symptoms. This implies that patients did not view self-monitoring as an important element in diabetes management. These feelings and perceptions could explain in part why only a few patients in this study tested their BG at home. Al-Adsani et al. (2009) reported that three-quarters of the 5114 Kuwaiti patients with diabetes only did self-monitoring when they felt extreme symptoms.

In the previous sections, patients' perceptions of different aspects of diabetes management have been presented. Patients shared a number of misconceptions about diabetes and are now presented and discussed.

\subsection{Patients' misconceptions of diabetes and its management}

Many patients shared their beliefs and perceptions of diabetes in this study. Patients' perceptions affected their level of adherence to their management plan. In this subtheme, I discuss two main misconceptions expressed by patients: medications and the seriousness of the condition.

Negative beliefs about medications were held by one-sixth of the patients $(n=6)$ in my sample. For example, these patients' responses during observations and FG discussions showed that 
Metformin was associated with side effects among them; therefore, the prescribed Metformin was not taken regularly. The following dialogue illustrates this point taken from two FGs:

Female patient 5: The doctor told me I should take Glucophage, but I refused to take it because it decreases my weight and I say I stick to Amaryl. I don't know why my weight dropped from 85 to 75 in 2 years. Surely, not because of walking because of Glucophage (FG3).

Amer: Did you stop Glucophage or the doctor did that?

Female patient 5: No, I stopped by myself. I refused to take it, and I have started to follow a proper diet and walk, and I was getting skinnier then I got back to take Amaryl 4 mg due to high HbAlc. However, I didn't do HbAlc after taking Amaryl yet to see what the result is after Amaryl.

Male patient 4: I take medications, but I take pills in one day and the next day no (FG4).

Female patient 3: I decrease the amount of metformin that I take per day. For instance, if I take three times a day, I feel back pain, and it affects kidneys, so I take one or two tablets a day according to my comfort. When I experience back pain, I stop metformin for two days (FG4).

Adherence to the medication regimen was affected by experiencing side effects. Some patients reported feeling uncomfortable due to experiencing a number of unwanted effects of Metformin. Weight loss, back pain and kidney problems were the most common side effects reported by patients. A number of patients either refused to take Metformin or reduced doses due to its side effects. This implies a form of intentional non-adherence to their treatment plan. The perception that Metformin caused unwanted side effects was considered by these patients as a barrier that hindered their adherence to their management plan. Female patient 5 perceived that non-adherence to her medication regimen elevated her BG levels (HbA1c $=9 \%)$. However, for her, the side effects of Metformin outweighed its therapeutic effects which was also the case for P3 and P4. P3 mentioned later in the discussion that she developed kidney and eye problems as a result of high BG levels. During the FG discussions, I asked these patients if they told the doctor about their modification of medication regimen and all of them replied no. During observations, two patients discussed their non-adherence to Metformin with the doctor. Metformin may cause loss of appetite, weight loss and digestive disturbances (Seifarth, Schehler, \& Schneider, 2012; Migdadi et al., 2018). However, there are other factors which 
may also lead to losing weight such as depression, certain medications and infection (NHS, 2016c). These patients adjusted and modulated their medication doses on their own and without sharing their concerns with HCWs. HCWs may also encourage patients to discuss their concerns related to medications such as side effects. Concerns about side effects of the medications and fear from insulin injections affected adherence to medication regimen among patients with Type-2 diabetes in Jordan (Jarab et al., 2018).

A further example of misconceptions was a male patient who came to the clinic, and his BG readings were high, and the following discussion occurred between him and the doctor:

The doctor asked him: 'Why don't you take the diabetes helper?' (Metformin)

The patient replied: 'I will lose weight and if I take it I will lose more weight' (he was pointing to his belly) (Field notes).

Some patients were reluctant to taking Metformin due to their beliefs about weight loss and how this is perceived in the community. Though this patient was obese, he refused to take Metformin because he was unwilling to lose weight. This patient viewed weight loss as a serious and unpleasant side effect of Metformin, rather than as a factor which may assist with the control of his blood glucose levels. Losing weight was perceived by this patient as a signal of bad health which contributed to poor adherence to his medication regimen. This patient's beliefs were consistent with the positive cultural image of plumpness among most people in Jordan. This form of obesity is called 'cultural obesity', and for women, it means that a woman with large body size is viewed as being a symbol of beauty and an indication of fertility and healthiness (Helman, 2007). For men, it indicates a high socioeconomic status and good health (Madanat et al., 2008). These patients' concerns about side effects of medications were also reported by another study conducted among 20 patients in Kuwait. Half of the patients with Type-2 diabetes who were interviewed mentioned that they stopped or adjusted their medication doses to relieve the medications' unwanted effects (Jeragh- Alhaddad et al., 2015). Most of the HCWs ( $n=7)$ were aware of patients' perceptions of the side effects of medications. This example is typical of those responses:

Not following medication regimen is the most common problem here because of wrong beliefs about the bad effects of medications such as sexual problems or metformin may lead to kidney problems and joint pain (Physician. Interview 1). 
This physician mentioned examples of common patients' beliefs about medications. Adherence to the treatment plan was affected by patients' perceptions of medications' side effects and their short-term benefits:

Female patient 3: Even treatments that taken from the centre are not treatments. They only stabilise the situation, and the doctor tells you to follow the diet. If they are cure treatments, the diet will not be needed. If the treatment that we take from the doctor is a cure, they will not tell us that we should follow the diet which they should say this treatment cures you with no need for diet instead. They are only relievers or tiny stimulators to prevent any defect in the body.

Male patient 5: Insulin and tablets are temporary not for a week or a month. When insulin and pills are taken in the morning, they are only for one day. Insulin and tablets are not a treatment. They are a method of decreasing symptoms. I consider my blood sugar is normal.

Amer: So what is the last HbA1c reading?

Male patient 5: My HbAlc is $8.85 \%$ (FG 4).

These patients perceived that medications are only to relieve symptoms without any curable and long-time effects. They emphasised more the short-term effects of medications rather than the long-term benefits to avoid diabetes complications. Similar findings were reported by 29 Omani patients as they only acknowledged the short-term benefits of medications (Subhi et al., 2016). Currently, there is no cure for diabetes (Diabetes UK, 2018d). Male patient 5 considered his HbA1c reading as 'normal' though it is high. This patient's misconceptions contributed to exacerbating his condition as later in the FG he reported the development of complications such as neuropathy and retinopathy. Another female patient supported these patients' views about the short-term benefits of medications:

The treatment is the same with no difference from one doctor to another. Every doctor you visit he says to you 'You should follow the diet' or if you are overweight 'You should lose weight'. We as people with diabetes seek the benefits from someone (doctor) who says to us 'take the treatment and eat free' (Female patient 2. FG 4).

This female patient presumed that she did not gain benefits from the current treatment plan, and placed high expectations on medications to provide a cure for diabetes. Taking medications was preferred over other more demanding tasks such as adhering to a healthy diet and doing physical activity. This female patient wished if diabetes can only be managed by taking tablets. 
Another patient also talked about a machine to cure diabetes:

I heard the news there is the machine that is invented in America to remove diabetes? (Male patient 2. FG 1)

It was evident that there was a lack of awareness among patients that chronic conditions such as Type-2 diabetes cannot be managed and cured only by medications. Patients' responses showed how the medical model informed their perceptions by focusing more on cure and medications than lifestyle and behaviour change (Wills, 2014). Medications were considered by patients as a top behaviour priority in their list of diabetes management behaviours. Patients reported that they had control over their adherence to medications which could be referred to as 'perceived easiness' although not all adhered to their medication regimen. Therefore, they had more behavioural intention of following medication regimen than doing diabetes selfmanagement behaviours that require lifestyle changes and long-term commitment such as diet and physical exercise.

Furthermore, patients felt that medications should be sufficient to maintain good glycaemic control levels. Treatment in the medical model depends mainly on medicines which are considered as one of the main features of the medical model (Giddens \& Griffiths, 2006). These beliefs were also demonstrated by 29 Omani patients who viewed that medications should provide the solution and a cure (Subhi et al., 2016). However, most of the patients in my study believed that diabetes is a chronic condition and it could not be cured. A typical example of responses included:

There is no cure for diabetes, but if you abandon diet and sport, it will not give benefits to the disease (Male patient 6. FG 4).

Taking medications based on experiencing symptoms of diabetes was mentioned by a few patients $(n=3)$. A typical an example of the responses included:

When I work, I forget to take medications because I don't feel tiredness or thirsty or fatigue. Even during winter, I don't feel that I have diabetes at all and I don't take tablets for 20 days. I know if I feel thirsty, my sugar is high but when I don't feel thirsty I know my sugar is good, and I don't need tablets (Male patient 4. FG 4).

Lack of noticeable signs and symptoms of diabetes encouraged patient 4 to not adhere to the treatment plan. This patient's perceptions encouraged him to stop taking his medicines for a short period. These denial attitudes were therefore seen as a barrier that influenced his 
motivation to adhere to his treatment plan. The previous quote from male patient 4 showed that work activities were used as an alternative for tablets to burn extra calories, therefore, taking medications was deemed an unnecessary behaviour. However, physical activity is not enough to achieve good blood glucose levels. There are other diabetes management behaviours that patients are advised to follow. As is apparent, male patient 4 displayed a poor understanding of diabetes management behaviours which affected adherence to his treatment plan. Similar misconceptions were reported by a cross-sectional study that was conducted with 200 Saudi patients (Alsunni, Albaker, \& Badar, 2014). This study's findings showed that two-thirds of patients thought that oral tablets were not needed when the BG levels were normal. This denial towards diabetes were also held by another patient in my study. A spouse of a patient talked about her experience with her husband's denial of his diabetes:

Approximately ....6 years. He does not believe in medications, and he eats to a level which problems occur between us due to eating. He does not want to adhere, and he says 'I can walk'. I feel there is a problem in his eyes because he is using the phone too close although he is wearing glasses. I am worried that something will happen in his eyes (Spouse. Interview 5).

This spouse talked about her concerns that her husband might develop complications such as retinopathy. This patient was reluctant to adhere to the medications regimen; therefore, he refused to take medications. His behaviour was influenced by his perception that he can still maintain his daily activities so did not see the benefit of taking medications or adhering to a treatment plan could affect his diabetes. Patients with a low perceived susceptibility may develop denial attitudes towards the risk of developing diabetes complications. Diabetes complications take time to develop, and body cells are affected by high BG levels for a number of years before diagnosis (IDF, 2017), and in the interim, patients may actually be asymptomatic or have symptoms which they view as only being mild or an inconvenience.

Patients expressed their perceptions of the severity of diabetes. The onset age of diabetes and type of treatment were perceived by patients as indicators of the severity of the condition. Diabetes was regarded by a number of patients as a 'mild illness'"

If diabetes comes after the age of 50, it is normal. It does not cause complications, and it does not affect (Female patient 6. FG 1).

When diabetes occurs at the old age, it will be mild (Male patient 5. FG 1). 
What do you mean by Type-2? I don't know what Type-2 and 1 are. Mine is not serious. I only take Glucophage. When I don't care and eat too many sweets and other things HbAlc increases to 7 ..... but it never reached 8 and usually 7.5 (Male patient 4. FG 6).

Patients thought that Type- 2 is a mild form compared to Type-1 diabetes. Lack of noticeable signs and symptoms of Type-2 diabetes made patients think that diabetes is not a serious illness. P4 was not aware of which type of diabetes that he had despite managing his condition by oral tablets only, and that his diagnosis was also written on his appointment card. Furthermore, there was a common belief among patients that Type- 2 diabetes is only associated with old age, and therefore, it does not cause any complications. They perceived that diabetes had fewer complications when it occurred after the age of 50.

All types of diabetes can cause complications and there is no mild form of diabetes (Diabetes UK, 2018b). The type of treatment was also perceived as an indicator to assess the severity of diabetes. P4 viewed that his condition is mild because he only takes 'Glucophage' despite his HbA1c readings being high. Diabetes managed by only oral tablets was perceived as a mild condition. This implies that patients with insulin treatment had a severe form of diabetes and may also be a factor which led to their reluctance to start insulin therapy. Therefore, treatment mode was mentioned as an indicator of the severity of diabetes as patients who take oral tablets think their diabetes is not serious. These perceptions are similar to patients' beliefs in Malaysia that insulin is only given for severe diabetes (Hassan et al., 2013). These stereotyped beliefs about the severity of diabetes affected patients' attitudes and behaviours. Patients who had a high level of expectations that Type-2 diabetes is not serious were at high risk for denial attitudes and poor adherence to the management plan. Patients' optimistic views about the mildness of diabetes were unrealistic because diabetes complications develop slowly without recognition. According to the HBM (Becker et al., 1978), patients who perceive diabetes as not being serious and as having a low susceptibility to developing diabetes complication are less motivated to engage in healthy behaviours.

There was a poor understanding of the aetiology of diabetes among patients. Diabetes was perceived as a short-term condition (acute disease) that is supposed to be cured following taking medications only. These perceptions were also affected by the media that takes advantage of these patients to provide a 'cure' for diabetes. These wishing beliefs contributed to poor adherence to treatment plans and increased the risk of developing complications among 
these patients. However, these patients were aware of long-term diabetes complications, and they already had neuropathy and retinopathy.

Another example of poor understanding of the aetiology of diabetes was a discussion among different patients in focus group 7:

Male patient 3: If the blood glucose becomes controlled, can the patient stop taking medications?

Female patient 4: No.

Female patient 2: No, the doctor may decrease the doses.

Female patient 1: Once you have started to take medication, it is for the whole lifespan.

The above discussion showed that the male patient was not aware of the nature of chronic condition management. He thought that diabetes could be cured and reversed by taking medications. Other female patients disagreed with him, and they showed a better understanding of the management of diabetes. This group discussion provided a good opportunity for patients to speak and listen to each other, ask questions, exchange knowledge and help each other. Another example of poor understanding of the aetiology of diabetes was expressed by a few patients $(n=3)$ :

My Spouse and I do not know what we have to eat. How rice increases blood glucose, it is unbelievable!!!!! So these things that I should know. It is believable!!! That rice and bread increase blood glucose. The nature of diabetes linked with high blood sugar but you are talking about starchy food so people should know that either the patient or the people around him. So you have sugar why you shouldn't eat Mansaf (a famous Jordanian cuisine made of rice, yoghurt and meat) which doesn't contain sugar but there are fats and carbohydrates in Mansaf (Male patient 3. FG5).

This patient lacked a basic understanding of a diabetic diet. He did not know that all sources of carbohydrates are broken into simple sugar in the human body which in turn elevate BG levels. This patient referred to diabetes as 'sugar' disease; therefore, he associated diabetes with simple sugar only. Other two patients supported his view of 'sugar' in the following quotes:

Really I do not eat sweets, and I do not know how I got diabetes (Female patient 6. FG 1).

Yes, this point. How did she get diabetes and she doesn't eat sugar? (Male patient 2. FG 1). 
These patients associated with the cause of diabetes with a high intake of sugar. This perception comes from the word 'diabetes' and is translated by many patients in Arabic to 'sugar'. This male patient 2 wondered why she developed diabetes despite not eating foods with high sugar content. Therefore, it is a common myth that consuming food with high sugar content causes diabetes (ADA, 2017). Al-Maskari et al. (2013) found that two-thirds of 575 patients with diabetes in the United Arab Emirates preserved that excessive sugar consumption was the primary reason for developing diabetes among them. Patients' responses showed a poor understanding of the basic knowledge of diabetes.

\subsection{Summary}

This chapter has presented and discussed the findings related to the knowledge, attitudes and perceptions of patients regarding diabetes and its management. Patients reported the different ways in which they were diagnosed with Type- 2 diabetes. Most of the patients visited the health facility after experiencing signs and symptoms of diabetes, often for some time before seeking medical help. A few patients were proactive and visited the health centre to check their blood

glucose levels. Some were diagnosed opportunistically having attended a health care facility for treatment of another complaint. Stress and family history were perceived as causes of developing diabetes and reasons for poorly controlled diabetes.

Fatalistic beliefs were shared when the patients were talking about the causes of diabetes, the reasons for developing diabetes and their inability to control their blood glucose levels. Patients felt that diabetes is an inevitable condition due to stress and having a positive family history. Therefore, fate was used either a stress relief, an acceptance or an explanation of their inability to follow their treatment plan. Patients also associated fatalistic attitudes with religious beliefs and the power of God as illness is in God's hands.

Patients were aware of most of the signs and symptoms of diabetes as well as complications. However, there was a lack of awareness about medications and aetiology of diabetes among a group of patients. This lack of awareness resulted in a number of misconceptions regarding Type-2 diabetes as being mild that affected patients' adherence to their management plan. Some patients managed their condition based on their experience of symptoms, so if no adverse symptoms of hyperglycaemic they stopped taking medications. Some patients thought Type-2 diabetes was a mild form of diabetes. Most HCWs were aware of patients' beliefs and misconceptions about diabetes and reported that insulin therapy was perceived by patients as a 
last resort and as a sign of end stage of diabetes, which resulted in some patients' reluctance to adhere to insulin therapy. Other factors also influenced adherence to insulin therapy such as needle phobia and socio-cultural norms.

The influence of socio-cultural factors on patients' adherence to their diabetes management plan is discussed in the next chapter. 


\section{CHAPTER SIX}

\section{INFLUENCE OF THE JORDANIAN SOCIO-CULTURAL CONTEXT ON \\ PATIENTS' DIABETES MANAGEMENT PLAN}

\subsection{Introduction}

In this chapter, I discuss my analysis of the influence of socio-cultural factors on patients' diabetes management behaviours (diet, medications and physical activity).

There are three main subthemes in this chapter; the first subtheme is 'the social component of food' which discusses the social meaning of food and food traditions and customs in Jordan and how this impacts on seeking to maintain glycaemic control. The second subtheme is 'social factors influencing physical activity' which discusses social factors and norms such as lack of an exercise culture in Jordan, socio-cultural barriers which restricted in particular women from engaging in outdoor physical activities, social norms, and social embarrassment with physical activity that hindered doing exercise.

Finally, patients, their family carers and HCWs reported the use of plants and herbs as complementary therapies and the reasons why patients sought to use them are discussed. First I discuss the main findings related to the social component of food that emerged during data collection and analysis.

\subsection{Social component of food}

Within this subtheme, two main categories were evident; the role of food in the Jordanian sociocultural context and an emotional component of food that influenced patients' diet plan.

\subsubsection{The role of food in the Jordanian socio-cultural context}

Patients talked about the influence of family, friends and culture on their food preferences and the food choices that they made on a daily basis and during social gatherings. Engaging in social relationships usually included increased consumption of sugar-sweetened beverages such as fruit juice and foods that contain high levels of fats and carbohydrates. Therefore, these dietary choices affected the ability of patients to maintain adherence to the recommended diet. Home eating habits and the nutrition knowledge of family carers also influenced the foods that patients consumed. 
Approximately one-third of the patients $(n=11)$ talked about the influence of meal contents and the quality of food that was eaten either at home or provided to them by hosts, on adherence to their diet plan:

In the morning I have to eat a good breakfast to fill my stomach, so I will not be worried about not eating till 5 or 6 p.m. Eating one piece of bread with breakfast is necessary....... I cannot cope without eating bread and rice. We eat Mansaf and sweets during invitations (Female patient 5. FG 3).

The environment affects you such as we follow our families' habit that everything should be eaten with bread and the lunch rice, rice. Now if you have breakfast without bread, Ifeel I did not enjoy myself, and I feel hungry. Because in the food, if you do not feel happy while you are eating you will not eat. If I do not eat bread, I feel I didn't have breakfast, and I feel unhappy (Female patient 1. FG 6).

These patients described the traditional eating customs and food culture in Jordan as unhealthy habits that hindered their adherence to their dietary plan. Female patient 5 reported that eating bread helped her to feel satisfied and full during her long working hours. This patient whose HbA1c was $9 \%$ and had a positive family history of diabetes frequently talked about the inability to overcome the habit of eating bread and rice every day. She also mentioned that food intake was an integral part of social gatherings and traditional meals such as Mansaf (the national dish of Jordan, made of lamb, rice and dried yoghurt) were shared during social invitations.

Female patient 1 perceived rice and bread (carbohydrates) as essential components of the meal that made her feel satisfied and happy after eating them. All types of foods should be eaten with bread and eating bread in every meal is an essential traditional eating habit. Lunch should also contain rice, otherwise it is not considered an appropriate lunch. Although this female patient was aware of dietary changes for patients with diabetes, it was difficult for her to change her eating patterns. She reported that she faced difficulty in changing her family habits of eating rice and bread in most of the meals due to feeling unsatisfied. Additionally, other family members would not accept not having rice and bread several times a day. Bawadi et al. (2012) conducted a cross-sectional survey to explore the cultural eating practices among 5000 Jordanians. This study reported that that $4222(88.9 \%)$ of 5000 Jordanians eat bread with every meal and most dishes commonly prepared in the Jordanian cuisine are rice-based such as Mansaf (Bawadi et al., 2012). Baig, Benitez, Quinn, and Burnet (2015) also conducted a 
systematic review to explore family-based diabetes self-management interventions. This review identified that the lifestyle changes required for optimal diabetes self-management often conflicted with established family routines. Self-management behaviours may necessitate changing the types of food prepared and consumed in the home, which may affect family routines (Baig et al., 2015).

During my 80 hours of observations, 112 patients came alone to the centre, 47 patients came with family members and 13 patients sent their family members to collect their medications for them. I also found that approximately half $(n=18)$ of the patients who participated in the focus groups attended alone to the centre. These findings may suggest that family carers who did not attend with patients might not be aware of the dietary advice provided by the HCWs. However, all the family carers $(n=6)$ interviewed talked about their support for patients in making the dietary changes, although it is acknowledged that this sample of carers may be more involved in their relatives' care as they were interested and willing to take part in the study. By carers not attending the centre, it was a missed opportunity for health promotion as a diabetic diet is a healthy diet, and this is especially important for those who have a family history of diabetes as other family members will be more at risk of developing the condition.

Food preparation and cooking were usually undertaken by the male patients' spouses and carers who made the same food for the whole family:

So I have started with him the diet and food such as no Egyptian rice, I make American rice, low starchy food, the white rice is forbidden, and we started to use the wheat bread. Sweets, he used to eat konafa (a type of sweet) every week. It is forbidden to eat konafa, and we only eat konafa occasionally (Spouse. Interview 4).

Social and cultural customs surrounding diet shaped patients' food preferences and also contributed to a high blood glucose level among these patients. People in Jordan tend to consume a lot of a famous Jordanian cuisine called 'mansaf', and it is considered to be integral to specific occasions and celebrations such as weddings, funerals and graduation ceremony (Jordanian National Strategy, 2011). Lunch is considered the main meal of the day and generally contains rice, bread and vegetables and sometimes meat (Madanat et al., 2008; Bawadi et al., 2012). Eating bread at breakfast is also common among people in Jordan. Therefore, rice and bread are essential components of the meal that made it difficult for patients to change their eating habits (Bawadi et al., 2012). 
Another challenge for patients was not only the quality but the quantity of meals that were eaten with family members or relatives. Two HCWs, one male patient and a patient's spouse talked about patients with diabetes sharing undifferentiated plates of food as a common social activity which mainly resulted from social habits and in some cases due to a low income:

The patient got invited with other 4 or 5 people or even two persons, and it is unbelievable that the people would make a specific meal for this patient with diabetes. People make food for all people, and the patient is forced to eat this food so either he eats a bit and stops or he eats with people (Dentist. Interview 8).

For example, some people have to make the whole cooker of rice because this family consists of 7 people and the father is not able to buy this, and he cannot feed his kids grilled food or meat, even vegetables (Spouse. Interview 3).

Regarding food and because of life circumstances, we cannot make two meals at home, so I eat half or two-thirds of the meal (Male patient 4. FG 4).

These participants mentioned factors contributing to plate sharing in Jordan such as the family structure with many members, the cost of food and strong family and social relationships within the Jordanian society. The dentist interviewed mentioned that families and hosts made one large plate of food such as rice and meat for many individuals to share regardless of people with diabetes. Eating separate meals was believed to be socially isolating and expensive. Therefore, it was difficult for patients to eat a different meal or separately from people which would have been considered as being inhospitable.

Male patient 4 whose monthly income was between 500-1000 JD (average income in Jordan is $455 \mathrm{JD}$ ), who had children at school and his spouse, also mentioned that financial factors forced people to make one type of food to overcome the cost of making different meals which he considered as a barrier. The above patient's spouse perceived that patients' low income and family responsibilities contributed to patients not being able to afford healthy foods that required more money such as fish. Most of the patients in this study were married and had families for whom they were responsible. Approximately two-thirds of patients had a monthly income that was less than 500 Jordanian Dinar (approximately $£ 500$ ). According to the survey conducted by the Economist Intelligent Unit, the city where the study was conducted was ranked as the most expensive city in the Arab world in 2018 (Aljadid, 2018). Therefore, cost, quality and availability of healthy foods influenced patients' ability to maintain a diabetic diet. 
Sharing the same food with families encouraged patients to consume more food with a high amount of carbohydrates such as rice and bread. Therefore, the home food environment negatively influenced patients' self-control and prevented them from taking control of their diet plan. These patients and their family carers perceived that a diabetic diet is a complex dietary modification that they were not able to follow. They were overwhelmed by the influence of diet as a dominant cause of high BG levels even though a diabetic diet is a healthy diet for all family members (Diabetes UK, 2018e). Therefore, patients and their family carers can eat the same appropriate diet that matches patients' needs. During my observations, doctors spent most of the consultations talking with patients and their carers about the importance of diet and diet modification. However, the doctors did not offer practical advice, and a few patients were referred for more specialist help with their diet. The average consultation was also 10 minutes, so limiting the time available for health promotion.

Sharing of food and communal eating also posed a barrier to the diet changes required for patients with diabetes. Therefore, it was difficult for patients to estimate the right portion size of served food which can be useful for them to help manage their BG levels and improve glycaemic control (Barnard, Levin, Gloede, \& Flores, 2018). A male patient reported that the size of the plate was another challenge that he faced whilst visiting people:

Another problem was the size of the food plate as an example yesterday we visited people in Irbid (a city in northern Jordan), and they forced us to eat Mansaf (full of sugar) (Male patient 1. FG 2).

This patient felt obliged to eat more because of traditional practices of generosity. He used the phrase 'forced us to eat' to refer his embarrassment if he had not eaten the offered food by the hosts. Force-feeding in this study did not mean that hosts physically forced patients to eat food, but that cultural norms dictated eating the food offered. He indicated that the large size of the plate during social gatherings and occasions limited his ability to adhere to his diet. It is also inhospitable to leave food on the plate offered by hosts. This response encouraged other male patients in the focus group to discuss the size of the plate:

You do not mind with your family, but with people, you will not accept that. If your relatives offer you food with a small plate, you will not be happy (Male patient 2. FG 2).

Serving a small plate was perceived by this patient as a sign of disrespect and lack of generosity which went against the social norms of hospitality in Jordan. Serving food in abundance to guests was perceived as a central and essential social activity within the Arab culture. In Arab 
speaking countries, guests are served a lot of food in abundance, and the socialisation process usually requires the consumption of traditional meals consisting of rice and meat (Alsairafi et al., 2016).

Not everyone felt this way as another patient disagreed with P2 that he did not feel offended if he has been served a small plate of food by hosts:

No, I do not mind. It never happened to me. If it happens, I will be happy (Male patient 3. FG 2).

The previous dialogue highlighted the influence of social norms regarding food on patients' attitudes towards portion size and size of plates. A sense of social obligation also forced hosts to serve food in excess to guests which added a social burden and pressure on patients to eat more food than was recommended. The above responses showed that patients need to learn skills to be able to refuse food sensitively. However, many patients with diabetes may not tell those outside the family that they have the condition which is explored in chapter six (section 6.2.2). Therefore, patients with diabetes tried to adhere to a healthy diet in a social context which mitigated against this. In a previously reported study, Bawadi et al. (2012) mentioned that when Jordanians invite guests on dinner, and as a sign of generosity, 93\% $(n=4423)$ of 5000 participants offer large portions, and 91\% $(n=4341)$ of the study participants keep insisting on people having more food.

In the Jordanian social context, hospitality and food consumption play an important role in the offering and receiving of food which can be understood within the context of what Mauss referred to as the gift relationship (Mauss, 2002).

The gift exchange term was developed from the North American Indian concept of 'potlatch' which is translated to mean "a system for the exchange of gifts" and 'to feed, to consume' (Mauss, 2002, p. vii). There are three obligations of potlatch; to give, to receive and to reciprocate. Mauss (2002) reported gift exchange practices among two different population groups. Potlatch in the North American context was extreme as a rule always to return more than was received if not then there was a loss of honour. In the Melanesian population where the potlatch was a less extreme form and less competitive because there the totalized giving does not presume competition between giver and receiver (Mauss, 2002).

In the context of the gift relationship, hospitality was perceived as a sign of honour and gift giving to guests; therefore, guests had to accept these gifts. There were two types of obligation: 
the obligation to give food to meet the social norms of hospitality in Jordan by hosts and the obligation to receive food by guests which are understood through applying the gift relationship (Mauss, 2002). The hospitality in this study was perceived regarding gift exchange. Hosts had to offer food and guests were committed to consuming it during the social gathering.

In addition to social norms regarding food in Jordan, there could be other explanations for the above patients' responses. $\mathrm{P} 2$ who had been diagnosed with diabetes for more than ten years mentioned that he never attended any education programmes related to diabetes. While P1 and P3 who attended the previous educational programmes in the centre showed more awareness regarding their dietary requirements. P1 and P3 also reported that their monthly income was between 300-500 JD while P2 mentioned that his income was between 500-1000 JD. Therefore, having several portions of rice and bread per day which are cheap might also be attributed to low income and awareness.

The previous dialogues between patients showed that food habits are followed within the context of the social obligation regardless of the amount of sugar in the foods. Approximately a quarter of the patients reported that hosts took great pride and honour in showing hospitality and generosity to them. However, there was a variation in patients' responses to the host's hospitality. Three males and two females mentioned that they ate the foods offered by hosts though it increased their BG levels. These patients felt socially embarrassed and worried their social relationships might be affected by refusing host's hospitality. Therefore, they opted to show courtesy and politeness by eating a little amount of high fat or sugar food. The generosity of people caused feelings of embarrassment and guilt for these patients after eating foods that elevated their BG levels. These views are evident in the following quotes:

I do not like to upset people, so I drink the cup of juice (Fruit juice) (Female patient 3. FG 4).

They also serve me different types of sweets, and they say this is a small piece it is not going to affect you, but after I ate it I feel guilty (Female patient 1. FG 6).

However, another group of patients $(n=9)$ mentioned that they did not feel embarrassed to refrain from eating the offered food. Typical comments included:

I don't feel embarrassed (Male patient 2. FG 1).

No I told them I am a patient with diabetes and I don't eat it which it is normal (Female Patient 2. FG 4). 
No, I don't feel embarrassed. I don't drink it, so I refuse it or ask for making one without sugar. Some people embarrass you (Male patient 1. FG 3).

It is normal I say sorry and refuse the food (Female patient 1. FG 7).

A male patient mentioned that hosts perceived their hospitality as being more important than his dietary requirements and modifications:

Regarding the community is the fact, they are not considerate especially when you visit them the host wants to show his generosity, and he doesn't consider you have diabetes because he wants to show his generosity. So his generosity is more important than your health (Male patient 4. FG 6).

These comments were atypical from the responses from most patients. Hosts served and encouraged patients to consume a large amount of food as a sign of generosity and respect. These patients were frustrated with hosts' attitudes of social pressure to eat and inaccurate instructions provided by hosts during social gathering and invitations. Male patient 4 indicated that people offered food as a sign of friendliness and generosity to patients without taking into account their dietary requirements though they knew that he had diabetes. These social norms regarding food interfered with patients' efforts to follow their diet plan. Though patients tried to avoid eating foods with high sugar content, people hindered their willpower by verbally insisting that they eat. Similar findings were reported by 16 Omani patients who found that social interaction was a barrier to manage their diet effectively. These patients mentioned that they would receive negative comments from hosts such as "maybe you do not like our food" and 'eat and Allah will take care of you' if they refused to eat (Subhi et al., 2016, p. 3).

Four HCWs also reported that patients found it difficult to refuse the served food by hosts. The quote below from a doctor is typical of the HCW's responses:

It is shameful if the patient refuses to eat the offered sweets and says he is a diabetic patient so it is culture (Physician. Interview 1).

This physician was aware of the role of the socio-cultural context in Jordan in patients' adherence to a diet management plan. Three HCWs and a family carer referred to the generosity of the hosts as lack of awareness because of the increased food quantity and reduced quality or abundance of food: 
Yes, this society is a disaster in the lack of awareness. I am sorry but when they serve you carbonated soft beverages which are the biggest disaster for the patient with diabetes for all people. Then they say 'drink this cup it will not kill you' ....you make the patient frustrated for one million years. The patient says 'I cannot drink because I have got diabetes' then people reply the famous words 'this cup will kill you!! It will not kill you...drink it today'...NO, I do not want to drink it (Spouse. Interview 3).

This spouse perceived some aspects of social customs and norms regarding food as being a 'disaster' for patients' health. She gave an example of a dialogue that occurred between patients and a host who was insisting on feeding the patients against their will. She also talked about force-feeding attitudes and incorrect advice that caused frustration for patients. This spouse also mentioned that it was difficult for patients to refrain from eating unhealthy food. As is apparent, generosity was deeply embedded and a cultural cornerstone of Jordanian culture. There is a need to raise awareness of diabetes amongst the wider community and population in Jordan to seek to mitigate against a community perception that eating high-fat, high-sugar food is not harmful to patients with diabetes to seek to reduce the pressure on patients from the wider community.

Generosity and hospitality were also associated with fatalistic attitudes held by people in society. A further two patients talked about their experiencing fatalistic attitudes of their family members. These attitudes interfered with their dietary adjustment:

My husband tells me: 'eat everything, and it is God's will'. However, I eat reasonably such as I eat one spoon of rice instead of 4 spoons (Female patient 5. FG 3).

People force you to eat by saying God protects you (Male patient 1. FG 5).

These patients felt frustrated by the fatalistic comments from their friends and families who tried to force them to eat. These patients also felt that their family members impeded their efforts to adhere to a diabetic diet by disapproval and putting the responsibility on fate. Female patient 5 mentioned on different occasions during the focus group discussion that she followed the dietary requirements that she learnt from the previous educational programme. As previously reported (section 5.2.1) fatalism was grounded in religious belief among patients' family members and friends. However, the patients quoted above sought to combat other people's attitudes by adhering to a diabetic diet. 
Another female patient reported that patients should have the willpower to refuse the offered food that may elevate their BG levels:

In my opinion, the determination is the most important thing. I am your guest, and the other person who invites me should be considerate. For example, I eat a little bit of dessert or dark chocolate (Female patient 3. FG 6).

A sense of social obligation forced hosts to serve food in abundance to guests which added a social burden and pressure on patients to eat more food than was recommended. This female patient indicated that patients should not be committed to consuming the offered food during the social gathering. Her responses during the discussion showed that she had the willpower to refuse the offered food.

Social events and occasions are more prominent in the summer months as ten patients talked about the impact of the summer season on patients' diet plan. For example, a physician and a patient reported:

In the summer season, people consume a lot of grapes and figs, so the blood sugar is not controlled during the summer season. Attending weddings and family occasions are common during summer when people eat a lot of sweets on these occasions (Physician 1. Interview 1).

We go to 4 or 5 parties and eat Mansaf or sweets in the summer (Male patient 1. FG 2).

Four HCWs believed that patients had a higher BG level in the summer than other times in the year. These participants mentioned that people in Jordan have a habit of serving much food during social occasions such as weddings, funerals, festivals and invitations. During the summer, the majority of the food consumed during social activities was foods with high sugar and fat content. Abundance and availability of these types of food presented challenges for patients with diabetes to adhere to a diet plan. The patients were expected to eat what they had been served. Otherwise, rejecting the offered food might offend the hosts. Besides the social pressure and obligation on patients to eat, many patients expressed emotional feelings towards food. In the following category, I present and discuss the emotional component of food.

\subsubsection{Emotional component of food 'forbidden phenomenon'}

Eating habits go beyond calories, nutrition and relieving hunger; food temptation and lack of willpower related to food were reported by one fifth of patients $(n=8)$ as barriers to controlling 
their diet especially during social and family gatherings at home. Thinking that foods are forbidden can make adhering to a diet plan more challenging.

The fear of being deprived of many types of food due to diabetes discouraged two patients from adherence to their diet plan:

You should deprive yourself of everything to avoid rising your blood glucose levels. Sometimes you are hungry, and you wish if you can eat more such as you cannot finish a piece of watermelon because you say it is full of sugar, so I eat half of it. Though the other person can eat two plates of watermelon. So I feel disappointed because I can only eat a little bit, not more and we love food (Female patient 5. FG 3).

This patient emotionally struggled to be like other people who did not have diabetes. These emotions were mainly expressed by her regarding food. She felt discriminated against and disappointed because of the inability to eat more while the others were able to eat. These concerns about the impact of a diabetic diet on the patients' life also made this patient feel emotionally frustrated due to limited food choices. She felt that all foods would increase her BG levels regardless of her efforts to maintain a healthy diet. As is apparent, a poor understanding of diabetes management behaviours affected this patient's adherence to her treatment plan. These feelings were attributed to the difficulty of finding food that does not affect her BG and lack of conviction that dietary changes lowered her BG levels. A male patient agreed with this female's view about feeling unhappy about being deprived:

Life, you feel something is missing. Sometimes, you stop yourself or deprive yourself of eating the favourite food, but it is always something missing in the normal life. You do not live a normal life as other people do (Male patient 1. FG 3).

This patient who had been diagnosed with diabetes less than for five years felt he was living an incomplete life because of the inability to eat certain types of food. He was also disappointed by the ability of people who did not have diabetes to eat all types of food. He perceived that his quality of life was affected by diabetes management through being unable to eat culturally preferred food. Food was perceived as a sensitive issue for this patient. Food was more than just calories, and these patients were emotionally attached to food by expressing feelings of discrimination and deprivation. Therefore, he felt deprived of the dietary habits that he had followed over his lifetime. 
A patient's spouse talked about a family tension over the non-adherence with the diet and the overeating behaviour of her husband:

He feels I want to deprive him of eating. For example, I make one chicken, and I want to give him a small piece, but he feels I discriminate him from his children. He is so sensitive, and I am worried that he will develop complications (Spouse. Interview 5).

This spouse mentioned that her husband felt unhappy by dietary restrictions and advice provided by her. The fear of being discriminated against weakened her husband's self-control to eat an appropriate food portion. This patient was not able to resist food temptation which was perceived as a lifestyle restriction by him. There were also issues of power in the above quote as he ignored his spouse's advice and did not take responsibility for his own health. This spouse also tried to help him but this caused arguments, and she experienced feelings of fear and worry that her husband may develop complications. She perceived diabetes as a more serious disease than her husband did. These perceptions were similar to a qualitative study which interviewed 19 patients with Type-2 diabetes and 19 family carers in Finland to explore family members' experiences of everyday life in families with patients with diabetes (Rintala, Jaatinen, Paavilainen, \& Åstedt-Kurki, 2013). This study's findings showed that family members experienced different kinds of fear and worries more than patients and they were also afraid that complications might develop in the future (Rintala et al., 2013).

Feelings of deprivation were reported as being introduced by inappropriate instructions and advice provided by HCWs and patients' family carers:

The patient should have people to say to him STOP. Some people may not have control over overeating food (Spouse. Interview 4).

Patient's will and diet but the patient should not deprive himself of eating starchy food and sweets. For instance, the doctor tells a patient that sugar, rice, potato and bread are forbidden, so everything is forbidden. The patient says 'I can only drink water' so he will not follow the proper diet. For instance, when I ask the patient for a decreasing amount of rice, he will do it, but when I tell the patient that rice is forbidden, he will not listen to me. Another example when I say sugar is forbidden, I should provide alternatives such as artificial sweeteners or fruit sugar for patients who can drink tea without sugar. Artificial sweeteners and fruit sugar provide patients sugary taste and fewer calories (Nurse. Interview 4). 
The spouse in interview four talked about the role of the family in patient's food choices and diet control. She reported family conflict and tension over dietary instructions provided by the patients' family carers. She recognised that her husband should take responsibility for his own health and take control over his diet which in turn would lead to adopting a more internal locus of control (Rotter, 1966). Internal locus of control indicates the extent individuals feel that they have control over the event (Rotter, 1966). This spouse also practised controlling and threatening behaviours over her husband's eating habits to stop him from consuming more food. These findings were similar to a qualitative study conducted including 11 focus groups with 45 patients with Type-2 diabetes in the United States to explore barriers and facilitators to diabetes management (Mayberry \& Osborn, 2012). Patients reported that their family members who are too involved in diabetes management by practising nagging and threating behaviours created conflict and undermined patients' success in adhering to their management plan (Mayberry \& Osborn, 2012). Balanced and positive family lifestyle is important to foster and maintain health and wellbeing of family members (Wallace \& Kenkre, 2015).

The nurse in the above quote also perceived that the dietary recommendations provided by the doctors were impractical, unrealistic and unrelated to patients' food options. She gave examples of her role in providing realistic dietary advice that matches people's food options. This nurse had taken part in the previous health education programmes, and she talked about practical dietary advice that was delivered by her during these courses. Some of my observations in the consultants' rooms supported this nurse's view that doctors provided unrealistic dietary advice.

A patient eats rice and bread because he doesn't feel satiety, so the doctor advised him about slow chewing for 20 seconds every time he puts food in his mouth (Field notes).

Another female patient was annoyed with people's dietary instructions especially those of her family, and she did not like being told what to do:

I feel unhappy when people tell me 'don't overeat, be careful' especially my family or my son or my husband when they say 'this is not permitted'. The aim of that is decreasing the amount of eating, but the patient feels that his family don't want him to eat. When a person tells you don't eat something or don't eat grapes, you start eating it as intransigence. In some days, your body needs sugar, so you eat, and I may stay away from sweets, so I take sugar from fruits then a person comes and criticises you because it is full of sugar (Female patient 3. FG 4). 
This patient talked about her personal experience with what she viewed as inappropriate advice and comments from her family members. She perceived that her family carers provided an unrealistic dietary restriction without offering alternatives. She was frustrated with the advice that did not address her real-life situation; therefore, social relationships and the family's comments discouraged her from following her diet plan.

As a result of the social food environment, five patients shared another concern of embarrassment by avoiding mentioning that they had diabetes due to a sense of shame as indicated in the following extract from FG 3:

People eat everything in front of the patient, everything available 'Ya haram' (showing sympathy) the patient is deprived. Ah, this is very important. Since they start serving konafa (a sweet) in the party or wedding or as you want... Ya haram, the patient with diabetes, has rights, and he is a member of the community (Lab technician. Interview 5).

Female patient 3: Same thing. I don't prefer to say that I have diabetes, I try to take control of myself. When I take the cup of juice, I drink a little bit of it. With discussion, people forget so it is not necessary to say I am patient so I take control of myself (FG 3).

These patients were reluctant to reveal their condition during social gatherings due to fear of social stigma and receiving what they saw as unhelpful comments from other people. They did not admit to having diabetes in front of people for fear of being stigmatised for having diabetes. As previously reported in this chapter, there was a sense of social obligation and commitment expressed by these patients to eat more food that was recommended for patients with diabetes during social gatherings. Female patient 3 showed respect to the hosts by drinking a minimal amount of the offered juice which explains how it is important to please hosts in the culture as a sign of good social relationships.

Three of the $10 \mathrm{HCW}$ s talked about the emotional role of food in their patients' lives. A typical comment follows:

People eat everything in front of the patient, everything available 'Ya haram' (showing sympathy) the patient is deprived. Ah, this is very important. Since they start serving konafe (a sweet) in the party or wedding or as you want... Ya haram, the patient with diabetes, has rights, and he is a member of the community (Lab technician. Interview 5). 
It is difficult of patients to follow a diet because they see all people eat sweets, sugar and other things but they eat food with no taste and no smell, so patients feel unhappy (Dentist. Interview 8).

The feeling of food deprivation turned to social isolation as patients were fearful of experiencing discrimination and so some limited their attendance at social gatherings and socially withdrew. These HCWs were aware of the social and emotional meaning of food for patients. These feelings were caused by the inability of patients to eat the served food whilst watching other people eat.

This section has highlighted the impact of the Jordanian socio-cultural context on patients' diet as an essential component of diabetes management behaviours. In the next subtheme, I discuss the impact of the social environment on physical activity.

\subsection{Social factors influencing physical activity}

This subtheme presents an analysis of the social factors that influenced physical activity among patients. All the patients discussed the type of physical activities they undertook and the factors which aided or impeded physical activity. In this subtheme, I discuss how social and cultural norms influenced patients' engagement in physical activity.

All patients were aware that physical activity is one of the diabetes management behaviours. However, approximately one-third of the patients $(n=12)$ reported that they did not engage in physical activity due to multiple barriers. Lack of an exercise culture in Jordan and social norms affected patients' efforts to engage in physical activity. A male patient talked about his negative experience of undertaking outdoor physical activity:

There is no available place for exercise. I am a teacher, and one day I was running in the street, and many of my students saw me and all the people at the school knew I was exercising outside then I felt ashamed. If each health club offers low membership fees for a group of patients and the trainer also shows them the appropriate exercises, $80 \%$ of this problem will be solved. Ministry of Health should make agreements with health clubs for making discount offers for people with diabetes such as membership fees should be $10 \mathrm{JD}$ (£10) rather than $50 \mathrm{JD}$ for membership for the sake of encouraging people with diabetes to exercise (Male patient 2. FG 2).

This male patient reported that living in a society where physical activity is not part of its culture discouraged him from exercising. He felt that school students and teachers at his school 
were unfamiliar with exercise taken outside. This suggests there was a lack of an exercisefriendly culture in the school educational system. There are a number of challenges that impede effective physical exercise in public schools in Jordan (Abu-Sobah, 2013; Al-Rajby, 2017). These challenges include the number of sports lessons that are completed in the school timetable should not exceed one lesson per week. Another challenge is the number of students; some classes exceed 43 students, which makes difficult for the students to achieve the desired benefit. There is also a shortage of infrastructure, for example, playgrounds, equipment, open spaces and safety zones in the schools (Al-Rajby, 2017). Additionally, there is a lack of community awareness of the importance of exercise, as more attention is focused on the students' scientific achievement than their participation in sport (Abu-Sobah, 2013). The prevalence of overweight and obesity among children and adolescents in Jordan were $21.6 \%$ and $10.2 \%$ in 2012 respectively (Musaiger et al., 2012). Therefore, there is a need for more public health campaigns to reduce the burden of obesity as a risk factor for developing Type-2 diabetes in the future. For example, Diabetes Prevention Week, Make Every Contact Count and Change 4 Life are examples of the public health campaigns that seek to improve health and well-being among the general public in the UK (Wallace \& Kenkre, 2015; Public Health England, 2018).

Male patient 2 indicated a number of reasons for not exercising. Lack of designated areas for exercise and poor urban planning hindered him to exercise outside. He also mentioned that he lost his motivation and avoided outdoor activities due to feeling ashamed of being identified by his students in the school. Therefore, he refused to do outdoor activities to avoid disdainful comments from people. Social and cultural norms competed with his efforts to do outdoor exercise. The Theory of Planned Behaviour (TPB) places more importance on social influences and perceptions about subjective norms (Hill et al., 1977; Ajzen 1991). An individual's intent to engage in behaviour is a result of perceptions about subjective norms with respect to how others would view the individual if they knew if the individual engaged in the behaviour. This male patient considered the perceptions of what other people would think about his engagement in physical activity. He evaluated not only the advantage of engaging in physical activity but also what others would say about his engagement in physical exercise. Therefore, he behaved in a way that other people thought was appropriate as a result of normative pressure to the detriment of his own health as he was overweight.

This patient also talked about inaccessibility to gyms and health clubs, and he suggested that health clubs could offer discount membership fees that may encourage patients with diabetes 
to do physical exercise. The financial factor limited his accessibility to joining health clubs. In the United Kingdom, many general practitioners prescribe exercise on prescriptions as a treatment for different medical conditions including chronic illnesses (NHS, 2016a). This could be a possible initiative for Jordan if the insurance companies would cover the cost of gym membership.

A nurse talked about the reason behind a lack of exercise by women due to socio-cultural norms in Jordan:

It is difficult telling young or old women to join the gym or exercise outside due to culture so that walking fast for 15 minutes at home is enough to burn calorie (Nurse. Interview 2).

This nurse mentioned that women faced disapproval from the Jordanian society to join health clubs or to exercise in public places. Therefore, female patients had to find other exercise options that they could engage in. Socio-cultural barriers restricted the women to engage in outdoor physical activities such as jogging and running.

Additionally, most of the sports facilities in Jordan are accessible only for men, and only a few sports clubs allow limited times for women to exercise. This issue is also considered as a barrier for women in other Arab countries (Al-Kaabi et al., 2009; Badran \& Laher, 2012; Alrahbi, 2014). Two of the female patients mentioned that they walked outside in public places to keep themselves healthy. Walking is an acceptable activity in the Jordanian context. None of the female and male patients reported joining health clubs or doing regular exercise. The older age of the patients interviewed such as a third of the patients were aged over 60 years $(n=11)$, and approximately half of patients had a BMI greater than 25 may also have hindered their ability to do physical activity.

Approximately half of the patients $(n=16)$ indicated their commitment to regular physical activity and walking was the main physical activity reported. For example:

I walk 20 minutes a day around my house, and I go back and forth to mosque five times a day (Male patient 4. FG 6).

Walking was the dominant outdoor form of exercise and mainly done by walking the short distance to work or to the mosque by men for prayer five times a day. Attending prayers at the mosque is the religious obligation for men, but women can usually pray at the mosque on Fridays. Walking was considered the most suitable activity for these patients. Darawad et al. (2016) conducted a cross-sectional study which explored exercise behaviours among 115 
patients with Type -2 diabetes in Jordan. This study's findings revealed that 51 patients reported walking was the most common exercise (Darawad et al., 2016). However, walking alone is not sufficient to meet WHO recommended physical activity levels. The recommended physical activity guidelines for adults are 30 minutes of moderate to vigorous intensity exercise per day on five days and muscle-strengthening exercises on two or more days a week (WHO, 2011).

Four female patients considered housework as an alternative to outdoor physical exercise as indicated in the following quote which is typical of the female patients' responses:

I do physical activity within the house, and I don't go outside like before. All of my activities at home (Female patient 3. FG 3).

This patient perceived that completing family oriented tasks and duties within the home were enough to maintain a healthy weight. She, as well as the other three female patients considered these incidental activities as physical activities that fulfilled part of their diabetes treatment plan.

In the previous section, I discussed the social factors affecting physical activity among patients. Another Jordanian socio-cultural aspect that was mentioned by most of the patients, their family carers and HCWs was the use of complementary therapies. It is important to mention that the types of complementary medicine that participants were referring to were herbs, spices and plants. The term complementary therapies refers to a group of health care practices that are not part of that country's own tradition or conventional medicine and are not fully integrated into the dominant health-care system (WHO, 2014). The use of complementary therapies is discussed now.

\subsection{Complementary therapies}

Most of the participants reported experiences of patients using complementary therapies, especially herbs and plants for the management of diabetes. In the following category all herbs and plants that were used as reported by patients, their family carers and HCWs are discussed.

\subsubsection{Types of herbs and plants}

Most of the herbs that were used by patients are reported in the scientific literature to have hypoglycaemic effects and anti-diabetes effects in the management of diabetes. For example, okra, onion, garlic, cinnamon, olive leaves, fenugreek and germander have beneficial 
properties such as improving insulin secretion, lowering fasting blood glucose, increasing glucose utilisation by body tissues and delaying gastric emptying of carbohydrates after eating (Sinjab \& Youssef, 2014; Ahmad, 2016; Diabetes UK, 2018c).

Okra (Bamia in Arabic) was reported as the most common plant used by a fifth $(n=4)$ of the patients who reported using plants. Okra or ladyfingers was used mainly to alleviate symptoms of diabetes such as itching, stomach ache, fatigue and high BG levels. Okra improves digestion, constipation and energy levels (Diabetes UK, 2018c).

A male patient talked about an okra preparation recipe that he reported was used by many patients:

The Bamia is mentioned since you marinate pieces of Bamia in water then you drink it. Many patients support that, and they used it for decreasing blood sugar (Male patient 1. FG $1)$.

This patient mentioned that his grandmother told him about this traditional remedy, so information about herbs is being passed down through the generations. During my observations, this recipe was mentioned by a few patients who reported that immersing okra in water overnight then drinking a cup of okra water on an empty stomach lowered their blood glucose levels. An experimental study was conducted in Bangladesh to explore the effects of okra on the absorption of metformin from the gastrointestinal tract in rats (Khatun, Rahman, Biswas, \& Islam, 2011). The study findings showed that okra reduced uptake of intestinal glucose in fasting rats, but it also decreased absorption of metformin in diabetic rats after meals. However, patients who used okra in this study mentioned that okra water was taken on an empty stomach. I was unable to identify any studies conducted on humans to explore the effects of okra on glycaemic control in patients with Type-2 diabetes.

Other commonly used herbs were cinnamon, artemisia and rosemary, used by three patients. Neither rosemary nor Artemisia was listed as main plants used by patients with diabetes in the previous studies in Jordan (Otoom, Al-Safi, Kerem, \& Alkofahi, 2006; Wazaify et al., 2011). I was unable to locate any previous studies that revealed beneficial effects on glycaemic control for rosemary or the use of rosemary in the management of diabetes, although it is known to raise blood pressure; therefore, lack of evidence about rosemary might put patients at risk of developing complications. A male patient replied to a female patient who used rosemary to treat diabetes: 
The rosemary that this sister talked about it increases blood pressure (Male patient 2. FG $3)$.

Rosemary may increase blood pressure levels and it may interfere with the action of hypotensive medications (Steven \& Ehrlich, 2009; Sayorwan, 2013). This male patient, a university graduate (aged 61-70 years old), was one of the most educated patients in this study who had also attended the previous education programme provided in the comprehensive health care centre. His responses during the discussion showed that he was aware of many aspects of diabetes management. He also accurately calculated his BMI as part of the sociodemographic questionnaire.

Artemisia extracts lower postprandial BG levels ( 2 hours after a meal) by acting like $\alpha$ amylase inhibitors medications that delay digestion of carbohydrates and conversion of complex carbohydrates to glucose (Awad, Seida, Shaffie, \& El-Aziz, 2012). Therefore, taking Artemisia and anti-hypoglycaemic medications together after meals could place patients at risk of hypoglycaemia. In addition to beneficial effects on glycaemic control, Artemisia was believed to improve food digestion and abdominal pain:

My husband believes in plants and herbs. For example, when he eats Mansaf he should take Hasa Alban (Artemisia) to aid digestion, lower sugar and relieve bloating (Spouse. Interview 5).

This spouse reported that her husband did not take medications and he only managed his condition by taking plants and herbs such as Artemisia. She reported during the interview that her husband did not attend his medical appointments in the health centre and the medications were collected by her. The doctors were not informed that he did not take the prescribed medications and his HbA1c was $11 \%$. Therefore, adherence to his medication regimen was negatively affected by the use of herbs, and in this case, he used plants and herbs as a substitute as opposed to complementing prescribed medication as reported by this patient's spouse. Reviewing the literature identified that the beneficial effects of Artemisia on glycaemic control were only tested in vitro. While literature showed that cinnamon regulates blood glucose levels and increased insulin sensitivity among patients with diabetes (Diabetes UK, 2018c).

In addition to using herbs as a single entity, different herbal mixtures/recipes were reported to be used by one-third of patients $(n=6)$ to treat diabetes and improve the function of the pancreas. A typical comment included: 
They also say olive leaves when the water boils you add olive leaves then you marinate till morning when you drink a cup before eating because it improves the pancreas then cure diabetes (Female patient 1. FG 8).

My study findings identified 19 different types of herbs and plants reported to be used by patients. Six of these plants have been mentioned in the Holy Qu'ran as well as by Prophet Muhammad; ginger, olives, fenugreek, garlic, germander and onion.

I do not have enough information about herbal medicine, but Prophet Muhammad mentioned the Black Seed (black cumin or fennel) and emphasised its therapeutic benefits (Son. Interview 2).

This means that using plants was also influenced by Islamic traditions and religious beliefs. Indigenous Arab and Islamic Medicine incorporate healing practices such as medicinal plants that emerged from Islamic religion, the medicine of the Prophet (also known as Tibb Nabawi in Arabic) and cultural norms of the inhabitants (Alrawi \& Fetters, 2012). Tibb Nabawi is defined as a collection of the Prophet's public health advice such as avoiding overeating and avoiding a patient with leprosy (Albinali, 2013).

Albinali (2013) noted there was general disagreement among historians about which name should be used to call the medicine during the golden era of the Arab world. Some historians called it Islamic medicine while others argued some non-Muslim physicians and scientists contributed to developing the Arab medicine. Tibb Nabawi was also called Islamic medicine by some scholars. Other researchers mentioned that Islamic medicine and Arab medicine are the same and they called it Traditional Arabic and Islamic Medicine (TAIM) (AlRawi et al., 2017). Al-Rawi et al. (2017) define TAIM as a system of healing practised since antiquity in the Arab world within the context of religious influences of Islam. This medicine consisted of herbs, dietary practices such as fasting, mind-body therapy such as prayer, spiritual healing such as prescribed prayers recited over food, and applied therapy such as cupping. For instance, 26 plants have been used in the management of diabetes mentioned in Arab medicine (Saad et al., 2005).

Table 4 lists all the plants and herbs that have been reportedly used by patients with diabetes in my study compared to the previous studies in Jordan. 


\begin{tabular}{|c|c|c|c|c|c|c|c|}
\hline $\begin{array}{l}\text { English } \\
\text { Name }\end{array}$ & $\begin{array}{l}\text { Local } \\
\text { Name }\end{array}$ & $\begin{array}{l}\text { Scientific } \\
\text { Name }\end{array}$ & $\begin{array}{l}\text { Number } \\
\text { of } \\
\text { patients } \\
\text { used } \\
\text { herb }\end{array}$ & $\begin{array}{l}\text { Listed } \\
\text { in } \\
\text { previo } \\
\text { us } \\
\text { studies } \\
\text { in } \\
\text { Jordan }\end{array}$ & $\begin{array}{l}\text { Listed } \\
\text { in non- } \\
\text { Jordani } \\
\text { an } \\
\text { studies }\end{array}$ & $\begin{array}{l}\text { Studies } \\
\text { including } \\
\text { diabetic } \\
\text { rats } \\
\text { In vitro }\end{array}$ & $\begin{array}{l}\text { Studies } \\
\text { including } \\
\text { patients }\end{array}$ \\
\hline Artemisia & Sheh & $\begin{array}{l}\text { Artemisia } \\
\text { vulgaris }\end{array}$ & 3 & No & Yes & Yes & \\
\hline Cinnamon & Kerfah & $\begin{array}{l}\text { Cinnamon } \\
\text { zeylaniucm } \\
\text { blume }\end{array}$ & 3 & Yes & Yes & & Yes \\
\hline Fenugreek & Helba & $\begin{array}{l}\text { Trigonella } \\
\text { foenum- } \\
\text { graecum }\end{array}$ & 1 & Yes & Yes & & Yes \\
\hline Garlic & Thoom & $\begin{array}{l}\text { Allium } \\
\text { sativum }\end{array}$ & 1 & Yes & Yes & & Yes \\
\hline Germander & Geádah & $\begin{array}{l}\text { Teucrium } \\
\text { polium }\end{array}$ & 1 & Yes & Yes & Yes & \\
\hline Ginger & Zanjabeel & $\begin{array}{l}\text { Zingiber } \\
\text { officinale } \\
\text { Rosc. }\end{array}$ & 1 & Yes & Yes & & Yes \\
\hline Okra & Bamia & $\begin{array}{l}\text { Abelmoschus } \\
\text { esculentus }\end{array}$ & 4 & Yes & Yes & Yes & \\
\hline Olive leaves & $\begin{array}{l}\text { Waraq } \\
\text { Zaytoon }\end{array}$ & $\begin{array}{l}\text { Olea } \\
\text { europaeal }\end{array}$ & 2 & Yes & Yes & & Yes \\
\hline Rosemary & $\begin{array}{l}\text { Hasa } \\
\text { Alban }\end{array}$ & $\begin{array}{l}\text { Rosmarinus } \\
\text { officinalis }\end{array}$ & 3 & No & No & No & No \\
\hline Sage & Meramyeh & $\begin{array}{l}\text { Salvia } \\
\text { officinalis }\end{array}$ & 1 & Yes & Yes & & Yes \\
\hline Thyme & Zattar & $\begin{array}{l}\text { Thymus } \\
\text { vulgaris }\end{array}$ & 1 & No & Yes & Yes & \\
\hline
\end{tabular}




\begin{tabular}{|l|l|l|l|l|l|l|l|}
\hline $\begin{array}{l}\text { White } \\
\text { Lupine }\end{array}$ & Termos & $\begin{array}{l}\text { Lupinus albus } \\
\mathrm{L}\end{array}$ & 1 & Yes & Yes & Yes & \\
\hline
\end{tabular}

Miscellaneous: Linseed, onion, apple vinegar, sour gum, barley, oil and lemon

As is apparent, participants reported usage of many types of herbs and plants and were mainly used as complementing traditional medication to alleviate symptoms. In the next category, I discuss the reasons and motivators behind seeking herbs and plants as reported by patients, their family carers and HCWs.

\subsubsection{Reasons for seeking complementary medicine}

There were variations in responses among patients regarding the advantages of using herbs to aid control of BG levels. Approximately half of the patients $(n=18)$ reported that they either used or were using herbs at the time of conducting the study. There were no gender preferences apparent in the use herbs which contrasts with previous studies in Jordan that female patients used herbal remedies more than males (Otoom et al., 2006; Wazaify et al., 2011).

There were a number of reasons reported as to why patients sought help from the herbalist. Feeling comfortable was the main reason for seeking herbs and plants as reported by four patients and one patient's spouse. A comment which is typical of these responses was:

I use rosemary, and I feel so comfortable especially as it stops any stomach-ache and sugar too. It makes me comfortable, and it lowers blood glucose when it is high (Female patient 3. FG 3).

This patient perceived the effect of herbal remedies as relieving stomach ache and aided in feeling comfortable. She felt satisfied by the ability of the herbs to alleviate the acute or shortterm symptoms related to diabetes and reduce her BG levels. However, rosemary is believed to increase blood pressure levels (Steven \& Ehrlich, 2009; Sayorwan, 2013). The ability of some herbs to lower BG levels also motivated another patient to believe that her diabetes could be cured:

Using germanders and rosemary by drinking them in the early morning when I wake up then I feel comfortable, and I feel diabetes has gone. I do not gain benefits from taking medications. Medications are useless (Female patient 1. FG 6).

By saying that her diabetes had gone, this patient referred to a feeling rather than an actual medical examination to determine whether her diabetes had improved or not. However, her 
HbA1c was $7.8 \%$, and she had already developed complications such as kidney problems. This patient felt satisfied and comfortable due to the ability of the herbs to help her physically and emotionally. She was dissatisfied with medications, and she perceived that her conventional medications were unable to provide her with as much relief and satisfaction as herbs did. Later in the discussion, this patient also mentioned that she mainly relied on using plants and herbs during travel and reduced the dose of medications. This suggests a form of intentional nonadherence to the treatment plan and lack of awareness of the management of diabetes. These feelings encouraged her to incorporate herbs and plants into her daily routine. She reported that herbs helped her more to lower BG than medications. This patient acted on her common sense understanding of diabetes and its management. From her perspective, she turned to using herbs and plants to fill the gap in her medication regimen. She validated the success of herbal usage through short-term outcomes such as feeling comfortable and decreased BG levels. These beliefs about the effectiveness of medications were also reported by another study conducted with 20 patients in Kuwait. A quarter of the patients with Type-2 diabetes found herbs were more effective than medications in lowering their BG levels (Jeragh- Alhaddad et al., 2015). However, the effectiveness of herbs in lowering the BG levels was only reported by participants and not confirmed by blood tests.

Patients felt that they gained more control over their own health by seeking herbs. They also felt satisfied by contributing to their own treatment and having an active role rather than relying only on the instructions of HCWs. Therefore, these patients reported psychological and physical comfort in using herbs. They also showed a desire for seeking more information about the management of their condition. They increased their awareness of other options for the management of their condition such as the use of herbs. Another factor in seeking herbs was patients' beliefs about the limitations of conventional medicine in treating diabetes. Therefore, using herbs was an indicator of an emerging interest among the patients interviewed in the holistic approach to health and increasing health-seeking behaviours.

Another two patients learnt from other patients' experiences rather than their family members about lowering their BG levels by using herbs:

I heard that a patient who controlled his blood glucose by using herbs (Male patient 3. FG 7).

A patient used a mixture of honey with other ingredients then he did not have diabetes (Female patient 4. FG 7). 
A female patient also perceived herbs as natural products that would not cause any adverse effects:

We trust herbs, and if herbs do not produce benefits, they will not be harmful (Female patient 1. FG 7).

This female patient believed that herbs are safe and free from side effects with no risk of harm due to their natural characteristics. She perceived plants and herbs as having minimal adverse effects which she considered as one of the main reasons for using herbs. This patient's perception is however inaccurate and may result in improper use and side effects from taking some herbs. Furthermore, many plants contain poisonous substances. Safety, undesirable effects, regulations, standards and preparations of herbs are examples of the main challenges that concern health care professionals and policymakers worldwide (WHO, 2014). Two Jordanian studies reported adverse effects of using plants and herbs. For instance, comorbidities, interactions with other medications, contamination, purchasing herbs from open containers and inaccurate dosing are examples of risks associated with herbal use in Jordan (Wazaify et al., 2011; Wazaify et al., 2013). None of the patients in my study mentioned that they used plants and herbs in the dosage form which placed them at risk of inaccurate dosage. Wazaify et al. (2013) reported that $13.2 \%$ of 53 patients with chronic conditions in Jordan used plants and herbs in pharmacological dosage form (Wazaify et al., 2013). A scarcity of supporting clinical data for herbs and plants in comparison to conventional drugs is also considered as the main challenge of using herbs (Bone, 2013). It is recognised that many conventional medicines are however derived from plants.

Another group of patients $(n=3)$ who had tried herbs mentioned that they had stopped taking the herbs due to a variety of reasons which included: complications, side effects, no benefits and a previous negative experience. Comments included:

I saw on TV on Egyptian channel treatment for 40 days which you will be cured. I bought this treatment and used from A to Z. It was only drinking water, and I did not gain any benefits (Male patient 5. FG 4).

I heard about olive leaves. There are two types of olive leaves; I used the green gelatine substance, and I saw on TV that it damages the kidneys and my blood glucose raised when I tested so I gave it up (Female patient $1 . \mathrm{FG} 8$ ). 
These patients used herbal products that were advertised on TV. Hoping to cure diabetes motivated this male patient to use unknown herbs. These perceptions were affected by the media that take advantage of these patients and false advertising to provide a herbal quick fix for diabetes. Female patient 1 also reported discontinuing taking some types of plants due to developing side effects caused by using the gelatine substance in olive leaves. However, she talked about a recipe including another type of olive leaves that she was using:

You take a handful of none-gelatine substance in olive leaves and when the water boils you add it. I have used it for 15 days, but I did not test my blood glucose yet (Female patient 1. FG 8).

This patient was not deterred from using herbs despite knowing that they may cause serious side effects.

Just over half of the patients $(n=20)$ did not believe in herbs and indicated that using herbs might result in side effects and complications. Examples of the responses were:

I don't believe in herbs even if I use them they will not lower blood sugar (Female patient 3. FG 4).

I never tried, and I do not believe in herbs because this is diabetes, not a headache just take Chamomile or ......no....this is diabetes. The gland that secrets insulin is damaged. The patient will not be treated with olive leaves or ....it is common that diabetes is the chronic condition for that I do not believe in herbs for this condition (Male patient 2. FG 5).

Male patient 2 understood that the pancreas was damaged which resulted in diabetes, therefore, herbs would not be able to reverse and cure diabetes. He associated herbs with their inability to cure diabetes. This 53 years old male patient, who was secondary school educated, had been diagnosed with diabetes for more than ten years. His responses during the discussion showed that he had gained much information about diabetes by attending educational programmes. He also mentioned that he attended a previous formal education programme in the centre:

I attended lectures for 10 years. Two diabetic specialists provided all information related to diabetes. So I gained much information about diabetes for over ten years (Male patient 2. FG 5).

A male patient was aware that many medications are produced from herbs and plants, therefore, seeking herbs was unnecessary: 
I read about it, but I do not use it. I used one or 2 times only. These medications are produced from herbs. $80 \%$ of medications come from herbs so why I will seek taking herbs because they have complications (Male patient. FG 3).

All HCWs ( $n=8)$ who were asked about their views on herbal usage did not encourage the use of herbs. All of them believed that herbs were not beneficial in the management of diabetes. However, these HCWs acknowledged that many patients used herbs alongside their treatment. Lack of health benefits and concerns about safety associated with herbs supported by research evidence were the main reasons why HCWs rejected the use of herbs:

We do not advise patients to use herbs. Patients can manage their condition by medications, proper diet and physical activity. Herbs have toxicity so that we advise patients to stay away from herbs. There is no herb or type of food having the ability to reduce blood sugar. There is no scientific evidence for using herbs and using of herbs may lead to complications such as kidney failure and liver inflammations. Patients should only take their treatment from the doctor (Dietitian. Interview 3).

This dietitian claimed there is a lack of sound scientific evidence of herbs and unknown effects on the body systems. She was mainly concerned with the safety, efficacy and unwanted side effects of herbs on other body systems. This HCW advised patients to avoid using herbs in previous health education programmes. Lack of awareness of the health benefits of herbs was also expressed by a nurse and lab technician:

We always advise patients not to use herbs, nor listen to their neighbours nor herbal experts because herbal medicine is empty medicine. Herbal experts are not smarter than pharmaceutical companies. If there are some benefits in one herb, the pharmaceutical companies will use it for making drugs for diabetes (Nurse. Interview 4).

This nurse mentioned that plants and herbs had no beneficial effects; therefore, patients will not be able to lower their BG levels and improve their diabetes by using them. She perceived that patients should only seek evidence-based treatment such as medications. This extract shows this nurse had limited information about herbs and there was also a conflict over the therapeutic effects of herbs between patients and HCWs in my study. Conflicting beliefs and unsatisfactory consultations resulted in non-compliance in using herbs among nearly half of the patients in this study. One of the main reasons for poor concordance with medical advice has been related to communication processes (Snelgrove, 2006). Ley (1982) proposed that patient compliance, now more commonly the concept adherence is used, can be predicted by 
the patient's degree of satisfaction with the consultation, level of understanding and accuracy of recall of information. Ley's model (1982) acknowledges the importance of effective communication and its relationship to compliance in providing health care services (Snelgrove, 2006).

Some patients believed that herbs were able to lower their BG level, but HCWs did not believe that. In 2003, the WHO estimated that $25 \%$ of all medications were extracted from plants (Helman, 2007). Metformin, a drug that was used by all patients in my study to lower their BG levels is extracted from a plant called Galega officinalis (Bailey \& Day, 2004). This implies that most of the HCWs followed the medical model and they were task-focused by focusing mainly on the prescribed medications without considering the other needs of the patients. This also explains how the medical model shaped the HCWs' perception of using non-prescribed remedies such as herbs. During my observations, the doctors and nurses did not ask patients about their use of herbs. Herbal usage was only mentioned by patients and their family carers during consultations which I observed:

A male patient came with his spouse to the appointment, and he had stopped taking a vitamin D supplement because he went to Saudi for four months and he used herbs instead. His spouse asked the doctor about using herbs, and the doctor replied 'I do not know' and did not explain further (Field notes).

Another HCW mentioned that patients might use herbs instead of medications to manage their BG levels:

Additionally, using herbs affects the treatment plan. For example, take this particular herb, mass media, as the patient stops his medications for using olive leaves or Lupine (Supervisor of Health Promotion. Interview 10).

This HCW mentioned that some patients abandoned their medical treatment for herbs. She perceived that using herbs hindered the patient's treatment plan. Patients might use herbs based on recommendations from the media. Most of the patients who used herbs used medications in combination with herbs as a complementary treatment. However, two patients (a male and female) used herbs as substitutes for medications due to their beliefs about the lack of effectiveness of medications. Therefore, usage of herbs affected adherence of these patients to their medication regimen. 
Five out of 38 patients mentioned that they had received advice from their doctors to avoid using herbs:

The doctor here told us during the previous educational programme: don't use herbs or other methods, they are useless (Female patient 5. FG 7).

All alternative methods were not recognised by this physician. This physician rejected the contributions that herbs and other types of treatment could make to a patient's health. However, many patients expressed a desire to help their condition, and they thought they benefitted from the use of herbs. This physician's perception was also supported by a nurse and another physician who talked about adding Metformin to herbal mixtures:

The herbalists grind the oral hypoglycaemic tablets with herbs. So the patient takes herbs, and he thinks the herbs lower the blood sugar, but in reality, the medication which is mixed with herbs lowers the blood sugar (Nurse. Interview 7).

This nurse attributed the health benefits of herbs and their ability to lower BG levels to the ground Metformin in the mixture. A male patient also pointed out this risky behaviour practised by herbalists (Attarah shops in Arabic) during the preparation of herbal recipes:

Male patient 2: Some people think they are experts and they make mixtures which are useless.

Female patient 5: They put the ingredient in it.

Male patient 2: This ingredient is Glucophage.

Female patient 5: They crush it and put it along with herbs (FG 3).

This dialogue from FG3 indicates that a few patients were aware of unsafe practices of some herbalists which might lead to patients inadvertently taking an extra dose of Metformin that placed them at high risk of complications. These unethical and misleading practices by herbalists took advantage of these patients as they reportedly sold their products by deception. Lack of awareness of herbs among HCWs, negative attitudes of HCWs towards herbs, and unethical practices of herbalists placed patients at risk of developing complications.

In Jordan, different names such as folk healers, herbalists and traditional healers are given to a person who is expert in preparing the appropriate recipes and mixtures of herbs for treating disease (Wazaify et al., 2013). Most of the local healers in the Arab World and developing countries including Jordan gained their experience from verbal instructions from their ancestors 
(Payyappallimana, 2010). Moreover, herbal knowledge is often inherited and transmitted across family members and generations without acquiring formal education (Saad et al., 2005; Wazaify et al., 2011). For instance, 55\% of 31 local healers in the West Bank acquired their knowledge about plants from their older family members by word of mouth (Azaizeh et al., 2003). Lack of a sound, formal educational background among herbalists may put patients with diabetes at risk of serious complications.

The discouraging attitudes of HCWs towards herbs hindered five patients to discuss their herbal usage with their HCWs:

Amer: Did you tell the doctor that you use herbs?

Female patient 1: No (FG 3).

Male patient 2: No, because the doctor only writes the prescriptions (FG 2).

Female patient 1: No (FG 8).

During my observations, HCWs did not ask patients whether they were using herbs. A few patients did discuss the use of herbs with their doctors. The fear of disapproval might hinder patients to inform HCWs about their herbal usage. There was also a lack of initiation from the doctors to include herbal use as part of a patient's medical history, especially when the patient took multiple pharmacological agents. Additionally, HCWs' lack of recognising that patients may be using herbs meant that this was not taken into account routinely when taking patients' history and prescribing medications. In a study of 166 patients with diabetes in Jordan, 15\% informed the doctors about using herbs (Wazaify et al., 2011). Wazaify et al. (2011) attributed these findings to a lack of patients' awareness of the harmful consequences of herbal usage and the fear of censure.

HCWs' responses showed how the medical model informed their perceptions and practice by focusing more on treatment and medications than other forms of healing such as herbs. Herbal usage was often ignored and although acknowledged by some HCWs was not routinely enquired about during consultations. HCWs believed that scientific medical treatment and knowledge were superior to the complementary medicine that was perceived as unscientific treatment. Medical treatment was perceived by HCWs as the sole accurate source of health information and treatment which contrasted with the perceptions of approximately half of the patients I interviewed. One of the barriers to primary health care is that the cultural beliefs of 
patients and HCWs may not be compatible, leading to poor health outcomes (Qidwai, Ashfaq, Khoja, \& Merchant. 2011).

Most of the patients who used herbs purchased their herbs from Attarah shops (traditional Arab pharmacies). These patients adjusted and modulated their herbal usage on their own based on the information received by herbalists without seeking medical advice. As there is evidence to show that some plants and herbs can influence BG levels, HCWs need to encourage more open discussion about herb usage during consultations.

The findings indicated that approximately half of the patients used complementary therapies, primarily plants and herbs, to relieve symptoms of diabetes which impacted on their quality life. Patients also used a range of different types of herbs and plants to enable them to cope with diabetes, but few informed HCW regarding their herbal use.

The socio-ecological health model (McLeroy et al., 1988) was useful for aiding understanding of the factors which influenced patients' experiences of Type-2 diabetes. Patients' relationships and interactions with immediate family and friends especially regarding family support, sources of information and home food environment were among interpersonal relationships (McLeroy et al., 1988). The traditional eating customs, food culture, herbal usage and lack of culturally sensitive health clubs were among the socio-cultural factors that influenced patients' diabetes management behaviours at the larger social system.

\subsection{Summary}

This chapter has discussed the social influences and consequences of management of diabetes for patients. The influence of the Jordanian socio-cultural context on diabetes management behaviours was discussed. The social meaning of food and food traditions and customs in Jordan were presented. This chapter also highlighted the social factors influencing physical activity which discusses social factors and norms such as lack of an exercise culture in Jordan, socio-cultural barriers restricted the women to engage in outdoor physical activities, social norms and social embarrassment with doing physical activity.

Finally, patients, their family carers and HCWs in this study also talked about the use of plants and herbs as complementary therapies and why patients sought to use them. Types of plants and herbs and the reasons for utilising them were also discussed in this chapter.

The following chapter will report the findings related to the challenges at the health care system and health care workers (HCWs) in the comprehensive health care centre. 


\section{CHAPTER SEVEN}

\section{CHALLENGES TO GOOD GLYCAEMIC CONTROL RELATED TO THE HEALTH CARE SYSTEM}

\subsection{Introduction}

One of the objectives of this study was to identify the challenges that hinder patients with Type2 diabetes living in Jordan to achieve normal BG levels. The findings presented in this chapter are primarily derived from the focus group discussions with patients, interviews with HCWs and through my $80 \mathrm{hrs}$ of observation in the centre. Participants reported a number of wideranging challenges that hindered management of diabetes at two main levels of care; the health care system and health care workers (HCWs) in the comprehensive health care centre which are discussed in this chapter.

First, I discuss the main findings related to challenges at the health care system level.

\subsection{Challenges related to the health care system}

Several challenges related to the health care system and its policies were mentioned, and the impact of those policies on service provision and patient care were also observed. These challenges such as medication insecurity and health insurance policies resulted in poor adherence to the management plan, multiple visits to the centre and patient inconvenience. Health insurance policies and medical exemption are discussed under the category of 'impact of health insurance'. Medication shortages were a barrier for most of the patients in this study which is discussed in the category 'medication supply problems'. The last category 'unavailability of laboratory tests' discusses the findings related to the challenges patients faced regarding laboratory tests. The data related to the impact of health insurance on patients' management plan will be presented and discussed now.

\subsubsection{Impact of health insurance}

\subsubsection{Medical exemption}

The national health insurance schemes and medical exemption policies were identified by patients and HCWs as a barrier that hindered adherence to patients' management plan. All types of health insurance and medical exemption provided in Jordan have been discussed earlier under the background section (section 1.4). 
Medical exemption means that the patient is only able to receive diabetes care at a specific centre. A female nurse considered this as a limitation because it affected patients' adherence to any treatment plan as indicated in the following extract:

Patient visits the centre based on his medical exemption. When there is a shortage of insulin or medications in the centre, the patient has to wait until the medications are available in the centre (Nurse. Interview 4).

In the case of a shortage of medications in that specific centre, the patients did not have the option to visit another centre to obtain their medications, so the patients had to make multiple trips to the centre to access all medications, or they ended up paying for the unavailable medications from private pharmacies which added financial burden on the patients. Additionally, further diabetes complications such as diabetic coma may develop as a result of poor adherence to medications. This exemption had to be renewed every six months by a written medical report explaining the patient's condition, and patients also had the opportunity to select the centre where they wanted to receive their diabetic care.

A female patient believed that medical exemption policies were a factor that hindered her accessibility to medication:

I only receive treatment in this centre because of my medical exemption so I cannot take medications from somewhere else. The medication for hypertension was unavailable. I take the plus one, and I do not take diuretic alone because I do not feel well when I only take diuretic alone. Amaryl was unavailable too, and I do not know what medications were not there. The doctor herself told me: do you want me to write the unavailable medications on separate prescription and you come later? In case of coming back to get the unavailable medications, there will be crowds waiting there (Female patient. FG 6).

These challenges demonstrate patients' adherence to their management plan was affected by national health policies. Medical exemption policies in combination with medication shortages contributed to the poor adherence to patients' medication regimen. In comparison to other diabetes management behaviours, medications were considered a high priority by patients in this study. My study findings were consistent with another cross-sectional survey in Jordan (Khattab et al., 2010) that found adherence to medications was more important than other diabetes management behaviours. 
A few patients $(n=3)$ talked about their limited access to have check-ups of other body systems and functions:

And our teeth hurt us and we do not know where we can go because they determine when they give you the exemption it is only for diabetes. I am not able to have my teeth fixed. If I let you see this area of teeth, it hurts me because I do not have the money to see the dentist. It is not a comprehensive treatment. I am not able to go to the ophthalmologist. ONLY for diabetes (Female patient 1. FG 6).

Lack of comprehensive care was another feature of the medical exemption policies. A few patients in the focus groups reported that they were not able to have their eyes, teeth or feet examined under medical exemption policies though they experienced complications for a long time or they were not aware of the consequences of diabetes. This female patient talked of her financial inability to visit the dentist because of her gum problems which can be attributed to sustained high blood glucose (BG) level, as the medical exemption only covered medications and laboratory tests. It is a known fact that oral health problems such as periodontitis are significantly associated with poorly controlled diabetes (Sanz et al., 2018). When diabetes complications develop, it is difficult for HCWs to reverse the damage to the affected organs. This national policy restricted patients from accessing other aspects of diabetes care. These policies only focused on one illness, diabetes, without considering its complications which support the concept of the principles of the medical model of care which focuses on treating disease rather than the whole person (Wills, 2014). In adopting a holistic approach, the patient would be seen as a whole system rather than a set of disjointed parts (Mele et al., 2010). The concept of 'holism' is well integrated within systems theory (Bertalanffy, 1968) in opposition to the reductionist approach in the biomedical model of health (Naidoo \& Wills, 2009). Secondary prevention of diabetes-related complications, for example, routine screening for diabetic retinopathy may also yield several benefits for patients and the HCS. Patients would be able to live a more productive life and have an improved quality of life, and secondary prevention could also reduce the financial burden of treatment of diabetes complications. In addition to the medical exemption policy, patients having multiple insurance policies were found as a barrier to achieving optimum BG levels and the impact of these multiple insurance policies on their management plan are discussed now. 


\subsubsection{Having multiple insurances}

Most of the patients in this study had access to another health care facility or dual insurance. In 2015, the Department of Statistics pointed out that a number of patients had two or more insurances which is considered as one of the reasons behind medication wastage and the high national spending on medications mainly for chronic conditions in Jordan (The Higher Health Council, 2015). During FGs discussion, patients reported visiting more than one health care facility:

Amer: Who visits more than one centre and takes his/her medications from other places?

Female patient 1: The health centre here and the National Diabetes centre (FG 7).

Female patient 4: I go to the Medical City, and there is support regarding diabetes (FG 1).

Male patient 4: Frankly, I like the National Centre. I visit it every three months, and they do a full check-up, and they test HbAlc every three months. They help you, and they give you medications and instructions (FG 6).

Patients who hold multiple insurances have the right to seek treatment in any of the health care facilities where the insurance is valid. Patients with multiple insurances were generally employed and had a higher income than those who were restricted to just the one centre under the medical exemption policy. Responses from patients showed that they had positive attitudes towards seeking treatment from other places. These positive attitudes were attributable to the instructions that were provided by HCWs in another place, availability of medical tests and medication security. Additionally, a lack of these facilities in the selected centre encouraged patients to visit different centres and hospitals. However, there were other implications of multiple insurances as reported by the laboratory technician in the following data extract:

The criteria used for testing blood sugar in the country are different from one place to another. For instance, in this centre, the blood sugar reading till 120 is considered a good level. While if you go to the National Diabetes Centre when the blood sugar is greater than 100 considered diabetes....there are different criteria, so the patient is confused (Lab technician. Interview 5).

This lab technician mentioned that the current diagnostic criteria for diabetes are not identical in the main health systems in Jordan. Every one of these sectors has its own guidelines and protocols for treatment including medications and laboratory tests which poses a challenge for 
patients and HCWs. Using one standardised criterion in the health care facilities in Jordan rather than using different sets of criteria as cut-off points for diagnosing diabetes may reduce patient confusion.

The findings suggested that conflicting advice and patient confusion were apparent among patients who had more than one health insurance and visited more than one health facility to obtain their medications or to receive diabetic care:

A patient was confused about the right dose of Metformin because he stayed in the hospital for implanting a pacemaker and the cardiologist there wrote different doses than the ones written in the patient's medical records (Field note).

When the doctors prescribe the medications for diabetes, they tell me to take them 30 minutes before eating while other doctors say during the process of eating or after eating. Sometimes, I forget, or I am confused (Female patient 5. FG 3).

This male patient was confused, and his adherence to medications was affected by receiving contradictory advice from different doctors. Also, this female patient felt upset and confused when she received different opinions from a variety of HCWs, and she was concerned about whose advice to follow. Patients may follow incorrect advice which may affect their treatment programme. Furthermore, a male patient received contradictory advice when he visited the hospitals to undergo medical procedures and interventions such as cardiac catheterisation:

A patient went to see the cardiologist in the hospital, and the cardiologist changed some of his medications and stopped Isoket retard tablets, so the doctor in the comprehensive care centre was surprised due to stopping Isoket (Field notes).

There was a conversation about Isoket to be added or removed, and the patient was not sure about that. This observation highlights that the cardiologist in the hospital changed this patient's medication regimen without considering his current regime recommended by the primary care physicians. Therefore, HCWs in the comprehensive centre were unable to monitor any changes in patients' medication regimen resulting from their visiting the hospitals. There were a number of explanations behind the conflicting advice from HCWs. Changes in patients' treatment plan might be based on the outcomes of the acute medical procedures in the hospital without seeking advice from other HCWs in the comprehensive centre which was considered as a lack of effective communication between HCWs and between primary and secondary care. 
Incomplete patient medical records was another implication which was attributable to patients switching from one health facility to another. During my observations, I found that many records were inaccurate and incomplete which posed challenges for other HCWs to take over management of patients' conditions:

A patient was hospitalised for cardiac catheterisation, but he did not bring the results for the doctor to see (Field notes).

A few patients' records have no laboratory results for the last 6 or 7 months, and patients left them at home (Lab technician. Interview 5).

Many patients did not inform the doctors in the centre about any changes in their treatment plan made by other doctors. During my observations, the doctors routinely asked patients whether there had been any changes to their medication regime or not. Some patients had laboratory tests or medical interventions in another health facility and did not bring the results with them during their visit to the centre. Laboratory results had to be kept by the patients enabling them to participate effectively in their treatment plans. I consider this policy as a positive feature to enhance empowering patients, but it requires patients to always bring their laboratory tests with them, which not all did. Several doctors wrote down the patient's laboratory results in their records based on verbal responses provided by the patient. Therefore, the doctors might change the patient's management plan based on results provided verbally by the patient which may not be accurate as patients may forget the right values of laboratory tests or they are unaware of these values. These factors contributed to poor continuity of care and incomplete patients' medical records. A few HCWs suggested that creating a shared computer system between all health care facilities and having one health insurance may stop the accumulation of medications, overdosing and improve patients follow up as illustrated in the following data extract:

MANY many. The same patient who comes to the centre he visits the National Centre, he visits the University hospital or in the military. The reason behind that is the fear of patient to securing the medications with no shortage of them. The solution for this issue is the union of the computer network. For instance, when the patient takes his medications from this centre people in the University hospital can see that on the computer. All are connected with each other (Pharmacist. Interview 6).

This pharmacist reported that a shared database would provide the opportunity for other HCWs to monitor patients' medication status and facilitate access to patients' medical records. 
Therefore, the doctors in the comprehensive care centre would also have access to any amendments and modifications in the patient's management plan instead of making decisions based on the verbal responses provided by the patients. That would also enhance the coordination between different doctors and may improve patient's satisfaction and adherence to treatment regimens. On the other hand, installing a single database may be expensive, takes time and needs regular maintenance. The policy in the centre did not allow for patients to keep their medical records with them:

The records are kept in the registry. The centre's policy keeps the records in the registry (Receptionist. Interview 9).

Keeping the medical records with patients may enable them to participate more effectively in their treatment plans (see figure 3). During my observations, the doctors asked whether the patients needed all of their medications to be written down on the prescription:

The doctor: Do you want all of your tablets to be written in the prescription?

A patient replied: We have plenty of this type of medication so please don't put it down on the prescription (Field notes).

As this data extract illustrates that either patients did not take their medications as recommended or they had alternative access to medications through multiple insurances. Sometimes the doctors offered free samples of medications provided by pharmaceutical companies that were not covered within the insurance or medical exemption for patients who were not able to afford to pay for them. In addition to challenges related to medications, a number of patients $(n=6)$ reported that they did not do self-monitoring blood glucose (SMBG) because of the cost of the strips which were not covered by health insurance. The insurance policies only provided these strips to children with Type- 1 diabetes. Therefore, a few patients were disappointed and stated that:

We are patients with diabetes too, and the health insurance should cover the strips to encourage us to test our blood sugar at home (Male patient 1. FG 5).

I wish if I have a glucometer. The strips are expensive, one box of strips costs $25 \mathrm{JD}$. If they sell it at discount prices, it will be acceptable. Testing blood glucose is necessary every week. I do not test my blood glucose for 2 or 3 months, and I do not test, so I do not know if my blood sugar levels are high or low. They give us medications so why they do not give us strips? (Male patient 3. FG 5). 
Patient 1 was not testing his blood glucose for long periods of time though he managed his condition through insulin. Insulin users are more likely to develop hypoglycaemia; therefore, SMBG is recommended to be part of their daily routine (Hillson, 2015). These patients wished that the Ministry provided free strips or at least offered discount prices on strips. By doing SMBG patients may feel more in control of their condition and participate effectively in their management plan. All patients in my study received blood glucose meters with 50 strips as a token of appreciation for their time and to encourage them to monitor their BG levels at home. However, a few patients $(n=4)$ mentioned that they test their BG levels regularly at home.

A few HCWs (n=3) suggested that all the health insurance systems should be joined as one national insurance system rather than patients holding more than one insurance:

I prefer that all the patients with diabetes hold one insurance and visit one specific doctor who patients trust and the doctor should be an expert in this field. I prefer one doctor who knows the patient's case. The patient feels confused and distracted from visiting more than one doctor. Every doctor gives you different information (Nurse. Interview 7).

That would yield benefits for patients and the health care system such as improving patients' adherence to their management plan, reducing the wastage of medications and lowering the pharmaceutical bill of the government. Furthermore, continuity of the patient-doctor relationship may be developed by seeing one doctor.

A review of The National Strategy and Plan of Action against Diabetes in Jordan revealed several objectives that were not accomplished as planned by 2011-2012 (MOH, 2012). When I asked a group of HCWs during participant observation about the strategic plan they were not familiar with its main objectives. The supervisor of Health Promotion was also dissatisfied when she was asked about the strategic plan as shown in the following data extract:

Ink on paper. So the achievable part is small, it is a disgrace in relation to the number of patients with diabetes who visit the health centres (Supervisor of Health Promotion. Interview 10).

One of the objectives of The National Strategy (2011-2012) was to increase awareness of diabetes amongst the community, but there was a lack of educational material in the centre:

We do not have posters, and nobody reads in this centre. If you give the patient brochures, he will not read, and it will be discarded in the dustbin later (Physician. Interview 1). 
Health promotion posters and brochures were not provided in the centre because HCWs perceived that patients were not willing or were unable to read. Furthermore, the health education programmes for patients with diabetes were stopped in the centre in 2016. Public health activities to increase the peoples' awareness about diabetes and its complications may encourage healthy behaviours among the public. Health promotion in the centre is discussed in detail in chapter eight. In the following section, data related to medication supply issues are discussed.

\subsubsection{Medication supply problems}

The main challenges regarding medication supply that were discussed by participants were medication shortages and changing medications brands. Medication shortages are discussed now.

\subsubsection{Medication shortages}

Multiple trips to the centre for obtaining all written medications in the prescription was mentioned and observed as a challenge related to the management of diabetes as indicated in the following extracts:

I got tired from standing in the queue, and then you do not find the medications and go home (Female patient 4. FGD 7).

Sometimes the patient comes to the centre, and they tell him there are no medications. The prescription is still in my pocket since Tuesday, and they told me most medications are not available (Male patient 1. FG 5).

Patients were disappointed and dissatisfied regarding travelling back and forth to the centre in order to obtain their medications. After standing in the long queue, the patient might be asked to return back another day to obtain medications that were out of stock or to go back to the doctor to write an alternative medication which necessitated a further wait. Both options resulted in inconvenience, especially for older patients, due to transportation issues and long waiting times. The discontinuity in medications supplies in the Jordanian health care system complicated patients' management plan as patients did not have a sufficient supply of the prescribed medications. This finding was supported by another previous Jordanian study. Alefan, Tashman, Mukattash, and Azzam (2014) revealed that long waiting times and multiple visits to health facilities resulted in inconvenience for patients and that medication shortages added more financial burden on the patients as they had to do more visits and may purchase 
medications from private pharmacies. Therefore, the patients started searching for alternatives to overcome medicine shortages such as securing medications from other sources or for those who could afford it paying for them from private pharmacies or using herbs instead (as discussed in chapter 6):

I go to another hospital, and there is support regarding diabetes. Tests are every six months, and they give me medications too (Female patient 4. FG 1).

The shortage of medications is not the health centre problem it is the Ministry's fault. This is my view. I do not think I go back to get Amaryl $4 \mathrm{mg}$ tablets that cost $10 \mathrm{JD}$, so I buy it from the nearest pharmacy, and I buy Aspirin 1.75 Jordanian Dinar too (Female patient 3. $F G 6)$.

These patients were seeking to maintain adherence to their treatment plan despite medication supply issues in the centre. It was also observed that a number of patients or their family carers informed the doctors at the time of writing the medication prescription that they had enough supply of a particular medicine. However, a number of HCWs showed disapproval regarding patients' attitudes towards hoarding medications from different places. A patient's spouse brought her husband's file, and the following conversation occurred between the doctor and the spouse:

The spouse: please, do not write Bufferin because we have three boxes of it.

The doctor (wondering): Did he stop taking it?

The spouse: No, we take medications from more than one place.

The Doctor: That is not right, don't do it again (Field notes).

In the previous conversation, the doctor was not willing to know why the patient visited other centres. As I mentioned earlier, patients tried to find another alternative for managing medication shortages by obtaining medications from other sources as they did not want to run out of medications.

The patients were seeking to maintain adherence to their treatment programme by ensuring they had sufficient medication despite a system which mitigated against providing continuity of medication supply. Taking medications on the scheduled time and in the right dose is considered as an essential part of diabetes management behaviours. Hoarding of medication was criticised and discouraged by a few HCWs. A pharmacist referred to this patients' 
behaviour as 'medication shopping' when the patients accumulated medicines collected from different places at home as a reserve. She considered this behaviour as a kind of wastage of medications and one of the factors which contributed to the shortages:

The connected computer system will stop the wastage of medications, and there will not be an accumulation of drugs with patients. When the patient dies, his family brings a whole bag of drugs that is used as a reserve which happened with us. Everyone brings a large shopping bag, and we ask him what are you doing with this? Ah (Pharmacist. Interview 6).

This pharmacist indicated that lack of awareness among patients and lack of a shared computer system were behind the accumulation of medications at patients' homes. However, from the patients' perspectives, they were seeking to secure their medications so they did not run out as they could not rely on the centre to always fully supply their medications. HCWs' perceptions are supported by a quantitative study conducted in Jordan to identify the reasons behind medication shortages in the health facilities from health care providers' perspectives (Awad, Al-Zubi, \& Abdallah, 2016). This study's findings found that human factors such as accumulation of medications by seeking medications from different sources by patients was one of the factors related to medication shortages. Several patients reported that the aim of the centre visit was only to obtain medications. The following patients' responses indicate their purpose for coming to the centre:

I visit the doctor for renewing my medications (Male patient 2. FG 2).

We just come here to take medications (Female patient 5. FG 3).

Here I come to take medications only (Male patient 4. FG 6).

Medications only (Female patient 3. FG 6).

These patients' responses were supported by observations as many patients were dissatisfied towards medication shortages:

The Doctor: Sorry, but many of your medications are not available in the pharmacy now. The medications will be available next week.

A male patient: I came here for nothing why they do not have my medications (Field notes). A few patients changed their medical appointment to come to the centre from the beginning of the month to mid-month to overcome medication shortages. However, the medication shortages existed at the time of the prescription refill. In addition to time and travel factors, 
patients had to stand in long queues waiting to receive their medications, as there were no seats available on the first floor where the pharmacy was located. After standing in a long queue, the patients might be told by the pharmacists to come back next week or go back to the doctor to write medication alternatives:

I am afraid of changing medications and unavailability of medications. I am afraid it is not the right medication, but we have to take it (Female patient 1. FG 8).

This patient felt unhappy and concerned regarding medicine alternatives. The quality of patient care in this study was adversely affected by shortfalls in public health policies regarding medications and changes to medication supply. McLeroy et al. (1988) mention that a patient's health is affected by national policies and regulations and any changes in the national policies imply changes in the patient's treatment programme. National health policies could be reviewed to maintain and to encourage patients to adhere to their treatment programme by ensuring the continuity of medication supply. The impact of medication shortages in public hospitals on patients has been investigated in a Jordanian study which included a sample of 357 patients (Alefan, Tashman, Mukattash, \& Azzam, 2014). This survey's findings indicated that patients' health outcomes were affected by shortages and patients also felt stressed and confused due to frequent changes in medications (Alefan et al., 2014).

Approximately a quarter of the patients $(n=9)$ in this study managed their condition by taking insulin therapy and oral hypoglycaemic tablets:

Why I should take my medications from two places. Instead of going to the hospital for one medication the centre here should provide it since the other types of insulin are available.

So the 24 hours of insulin should be available (Female patient 3. FG 3).

This female patient who had a medical exemption talked about her experience of collecting the long-acting insulin 'Lantus' preparation from the hospital. She indicated that insulin shortages were stressful and inconvenient for her because she had to do more visits to the hospital. Another aspect of medicine shortages mentioned by a few of the patients was that they did not receive the right amount of tablets at the time of the prescription refill:

When I went back home, I found I had only 24 tablets of Metformin instead of 30 (Field notes).

There is a doctor who writes all medications and another one who doesn't write all of them, so there is a mess in medications (Female patient 4. FG 8). 
A few doctors did not also transfer a number of medications to the prescription paper which resulted in the discontinuity in taking medications as prescribed. These medication errors may negatively affect the patient's health outcomes. Incomplete patient's medical records may also have an indirect impact on the accuracy of the written prescriptions. Another feature of medication insecurity was changing medications brands which is presented and discussed now.

\subsubsection{Changing medications brands}

A few of the HCWs talked about the impact of the national health sector policies regarding medication supply on the patient's medication profile:

Firstly, the system of medications brands in the Ministry as an example it is not always the same brand available or ..... may be available or the alternative one. For instance, this month the patient takes Amaryl (Glimepiride), the next month there is no Amaryl (Glimepiride) available, the patient takes Diamicron (Gliclazide) then you put the patient on the third drug. Based on what is in the tender, the patient should take the type of drug (Pharmacist. Interview 6).

Frequent switching from one brand to another based on the available tender contract in the Ministry meant patients did not receive the same brands of medication consistently. These medication supply policies resulted in inconvenience for many patients in my study. Any changes in the medication supply system in the Ministry of Health implied changing the existing treatment plan for patients which affected. in turn, patient's adherence to their treatment programme. Each part of the health care system affected another, and any defect in one part had consequences on other parts such as patient outcomes (Pearson et al., 2005). However, my study findings showed a variation in patients' responses in terms of receiving different brands:

The alternative doesn't bother me, but I like it has the same effect and gives the same results. I don't care about the colour or the type (Female patient 1, 45 years old, diploma. FG 7).

I am afraid of changing medications and unavailability of medications. I am afraid it is not the right medication, but we have to take it (Female patient 1, 65 years old, secondary school. FG 8).

My findings indicated there is a variation in patients' responses related to receiving different brands of medication based on age and educational level of patients. Older and less educated patients $(n=3)$ showed more willingness to maintain the same brands of medication than 
younger and educated patients $(n=3)$. It is acknowledged that the numbers are small so this finding is tentative. A few younger patients $(n=3)$ had no issues as long as the different brands had the same efficacy and produced the same therapeutic outcomes. Older patients might recognise their tablets based on the physical characteristics of medications such as colour or texture or appearance. For instance, patients referred to their medications as 'red tablet' or 'the rounded one' or 'a pill with strength 500' rather than their using actual names:

However, you know, I lost more weight by using the long tablet of Glucophage than the rounded one with the same strength. I lost 5 or $7 \mathrm{~kg}$ by taking the long one but no way to lose weight by using the rounded tablet, I put more weight on instead. I do not know.... Also, when they gave me the long tablet back, I felt happy (Female patient 2. FG 7).

A few patients $(n=3)$ experienced a negative impact because of receiving different brands in their management of diabetes. This was because the patients were familiar with the same wellknown brands and they had adapted themselves psychologically and physically to a particular brand. For instance, they perceived that the various brands of Metformin had different effects. The previous negative experience of the patient with the 'rounded tablet' suggested that different brands affected the patient's health. During participant observation, many doctors and pharmacists were trying to convince and explain to the patients who were dissatisfied with the change in medication brands that all brands had the same therapeutic effects with no difference. However, from UK experience, some brands of drugs with the same drug content cause more side-effects, for example, some statins result in more side-effects than others, and different statins produce different side-effects (NHS, 2016b). The Ministry also used different brands of the same medication to resolve medication shortages.

There was distrust expressed by a patient's spouse towards the quality of locally manufactured brand medications:

I put water on it then I crushed it...it is sand, it is not medication. Look at the texture of foreign medication and the texture of Jordanian medication, the texture of foreign medication is soft due to the ingredients but look at the Jordanian medication ... you seem to carry the stone. Unbelievable the medication here is bad (Spouse. Interview 3).

Size of the tablet, physical attributes, effectiveness and ingredients of these medications were criticised by a patient's spouse. She believed that components of drug products are low-grade quality in comparison with other western brands. Another study in Kuwait showed opposite perceptions towards the local brands. $75 \%$ of 20 patients with Type- 2 diabetes who were 
interviewed perceived that the local medication was better than the Western one due to freshness and lack of trust in the Western medications. Only a few patients indicated that the Western brand was more effective in lowering blood glucose than the local one (JeraghAlhaddad et al., 2015). It was difficult in my study to explore the quality of local brands, and it was beyond the scope of my study. However, the pharmaceutical industry in Jordan exports medications to many countries in the world including the USA and the EU due to its high quality and good reputation (Alefan et al., 2014).

Another feature of brand discontinuity in the centre mentioned by three patients was splitting the tablets:

Frankly, dividing the medications when they give me a $10 \mathrm{mg}$ tablet and I have to take 2.5 mg. This means I have to divide the tablet into four parts. It is so hard, annoying and psychologically uncomfortable. I feel I am not accurate to divide the tablet into four equal parts because each part may be more or less than the other so this thing is annoying. One time a pharmacist told me that the tablet loses its effectiveness when it is divided and put in the fridge (Female patient 3. FG 8).

Splitting the tablets meant dividing the medication pill into smaller doses for obtaining the right doses as required. Splitting the tablets was not welcomed by a number of patients $(n=3)$, and they felt stressed by it. The main concern reported by the patients was the efficacy and accuracy of medication doses and whether the dividing parts produced the same effect as the original one did. The patient may end up taking over or underdosing and as I mentioned earlier that taking the right dose is an essential component in the treatment plan. Food and Drug Administration in the USA (FDA) indicated that splitting tablets is not recommended practice unless it is written in the medication label information. Splitting the tablets into equal halves may not produce the same strength even though there is a midline in the tablet (NHS, 2011; FDA, 2013). Splitting tablets may result in uneven doses, patient confusion and inconvenience for patients:

For example, my treatment dose is $50 \mathrm{mg}$ dose, and the available dose is $100 \mathrm{mg}$ so I bought a special clip for medications and I keep it with me. So if it is a half of the tablet, I clip the tablet to be right and effective dose. Because what is available, available (Male patient 5. FG 8). 
This male patient bought a special clip for dividing medications which was not suggested or provided by the centre. Patients were actively seeking ways to maintain adherence to their medication regimen.

In this section, the findings related to the medication supply problems and their impact on patients' management plan as reported by participants have been presented. The national health insurance policies could usefully be reviewed to give the patients the option to receive their medications from private pharmacies as an example, instead of making multiple trips to the centre. According to the responses of the participants, many of the HCWs indicated that having a shared computer system would enable HCWs to monitor patients' medications status and facilitate access to patients' records (see figure 3). This may prevent medication dosing errors, duplication of medication and reduce patient confusion. The consistency of medicines brands and medication security are important factors in any diabetes management plan. Patients may feel psychologically comforted and reassured that the same appropriate brands are always available as they do not need to divide the tablets into parts. Furthermore, this may improve the quality and the potency of the medications as one of the suggestions made by participants in this study.

Laboratory tests are effective indicators to evaluate the adherence of patients to their management plan. However, there were challenges that patients faced in doing the necessary diabetic tests. These challenges are presented and discussed now.

\subsubsection{Unavailability of laboratory tests}

Data analysis revealed that participants experienced a number of barriers in relation to essential monitoring tests to aid in achieving good glycaemic control. These barriers are presented and discussed in this section. HbA1c test and other essential laboratory tests such as postprandial blood glucose test, lipids profile tests and liver enzymes were not available in the selected centre:

I go to the central laboratory multiple times, and it is crowded too. The bus takes one hour to reach there, and I wait 2 hours to do the test then they tell you the test is not available today or instead of doing one test come back and do all the tests at one time. This happened with me more than once, and I went back 2 or 3 or 4 times. So it is stressful. It happened with me (Female patient 2. FG 7).

I am not able to go there, and my children drop me here (Female patient 4. FG 7). 
Most of the unavailable laboratory tests in the health care centres in the city where the study centre was located are done in the central laboratory. Distance and transportation were often mentioned by most of the patients as reasons for delay or not doing tests in the central public health laboratory. The unavailability of essential tests in the centre was not welcomed by patients, and they also felt disappointed and stressed. Patients were also dissatisfied regarding the long waiting time and the boring routine in the central laboratory as they had to spend all of their day waiting to be seen. There was a lack of an appointment system in the central laboratory. Sometimes patients had to do multiple trips to the laboratory namely when they had many tests to be completed. Most of the patients were of an older age which is congruent with the aetiology of Type-2 diabetes, and they were not unable to attend the laboratory alone as they needed a family carer to accompany them every time they went. Many patients experienced other health problems such as hypertension, stroke and diabetes-related complications which influenced visiting the central laboratory. Another challenge was the unavailability of a number of tests due to shortages of equipment and staff in the central laboratory:

I take my medications from here and Lantus from the National Centre. Regarding tests, they write me tests that are not available here, so I go to the central lab. I have a hard time there because two-thirds of the tests are not there such as Iron so I am forced to go to the National Centre and pay money (Female patient 3. FG 3).

This patient felt disappointed as she went back and forth many times to the central laboratory or she had to visit more than one place for completing the requested tests. This might lead to fragmented care as patients had to visit several health facilities to receive appropriate care. In system theory, the health care system is composed of different parts, and these parts are interrelated and interconnected with each other aiming to achieve holistic care (Bertalanffy V, 1968), yet a number of patients in this study experienced a lack of holistic care. The unavailability of the tests also added a financial burden on the patients as they had to pay for tests in the private laboratories or incurred additional transport costs.

There was also a delay in doing tests caused by fear of needles or 'needle phobia' among a few female patients:

I have a fear of injection when they draw blood I feel dizzy. If they find another solution aside from drawing blood, I will do tests (Female patient 5. FG 3). 
This female patient reported that needles were painful and had her blood taken was a stressful experience. A nurse reported that patients might not have the desire to go to the central laboratory to do one test:

The patient should make two trips for getting the result of HbAlc in the central laboratory. Sometimes the doctor only orders HbAlc and patient feels lazy for travelling two trips to do one test (Nurse. Interview 4).

This nurse's account suggests that patients may not do the test which may negatively affect their treatment plan when they next came to visit the centre.

Most of the HCWs in this study were aware of the challenges that patients faced in relation to doing laboratory tests and the impact on patients' health outcomes:

HbAlc and random blood sugar are unavailable. Patients face challenges such as transportation and multiple trips in doing these tests in the central laboratory. Fasting blood sugar is only available (Dietitian. Interview 3).

Travelling is also not cheap to do the test (Physician. Interview 1).

Any changes in medication profiles of patients need frequent monitoring of blood glucose and comparing results for assessing patients' progress. For instance, the impact of changes in medications dosage and monitoring medication side effects are not achieved without laboratory results. A lab technician suggested that essential tests should be available in the centre to encourage patients to complete tests as required:

I wish if they provide us with HbAlc for patients because it eases and saves the patient from travel or from doing the test in the private sector, so we do it here (Lab technician. Interview $5)$.

Multiple trips, availability of family carers to accompany the patients (often older and frail patients), availability of the tests, lack of an appointment system, time factor and length of the patients' stay in the laboratory were factors that affected doing the laboratory tests among the patients. Providing essential tests in the centre is crucial and it may facilitate monitoring of the outcomes of patients' treatment plan. It is written in Jordan's National Strategic Plan (20112012) that all laboratories in the comprehensive health care centres would be at least equipped with the HbA1c test over six years ago. However, many essential tests such as HbA1c were unavailable in the centre during the period of this study. The centre is located a short distance 
away from most of the patients which would reduce travel expenses and time, and may encourage them to visit it multiple times for doing tests as requested. Provision of these tests at the centre would also mean that HCWs would have accessibility to accurate and complete records of laboratory test results for patients. Introducing an appointment system in the central laboratory may also be helpful in improving patient's satisfaction and also reducing the patient's waiting time to be seen by lab technicians.

In addition to the challenges related to the centre and the health care system, there were challenges related to $\mathrm{HCWs}$ which were reported by patients, HCWs, family carers and observed during 80 hours of observations conducted at the centre. These challenges are presented and discussed now.

\subsection{Challenges relating to health care workers (HCWs)}

This section highlights the findings regarding the impact of different aspects of staff shortages and poor patient-HCWs communication on patients' management plan. All patients had to visit the reception desk which was considered the first point of contact for patients during their visit to the centre. The following conversation was reported by a 65 years old female patient who had spent 5 hours in the centre, and she recounted her experience with the reception staff:

I (patient) asked her: Why didn't you give me the file?

She said (receptionist): Go upstairs and write a complaint to the management.

I told her: I am not here to complain, I am tired.

She said: The person who is expert in the registry he still didn't show up.

I told her: Please, find my record instead of finding other patients' records because I am dizzy and I have been in the centre since 7:30 am (Female patient 1. FG 6).

The reception desk was located on the first floor in the centre and it had two small windows. The patients had to stand in a queue waiting to receive their medical records which affected the patient's privacy in treatment. The average number of patients with chronic conditions who attended the reception desk to receive their records was 150 patients per day and this number increased during weekends. Only 2 or 3 reception staff were on duty with one receptionist at each window. In the registry, there were new staff members who needed more training which resulted in delays in providing records for patients and long waiting times: 
Honestly, when my duty is working on the patients' files, they don't stand up. The rest of the staff except one girl are new. So when they are new persons on the windows, and I get a problem to be solved the patient may stay 30 mins or more only in the queue. It is stressful (Receptionist. Interview 9).

The reception desk was located on the first floor in the centre, and it had two small windows. The patients had to stand in a queue waiting to receive their medical records which affected the patient's privacy in treatment. The average number of patients with chronic conditions who attended the reception desk to receive their records was 150 patients per day, and this number increased during weekends. Only 2 or 3 reception staff were on duty with one receptionist at each window. In the registry, there was new staff who needed more training which resulted in delays in providing records for patients and long waiting times:

Honestly, when my duty is working on the patients' files, they don't stand up. The rest of the staff except one girl are new. So when they are new persons on the windows, and I get a problem to be solved the patient may stay 30 mins or more only in the queue. It is stressful (Receptionist. Interview 9).

In the above quote, the receptionist, as an experienced member of staff was able to quickly locate patients' medical records, so that patients did not spend a long time in the queue. Due to a number of staff being new to the role, this resulted in delays in providing records for patients and as a consequence, longer waiting times. Staff shortages in the registry and lack of new staff competency also impeded the rapid transition of the patient to see doctors. Rapid transition of the patient through the registry would mean that a patient would spend less time in the centre which in turn would provide an opportunity for other patients to receive care (see figure 3 ). A few patients kept their medical records at home to overcome standing in the queue waiting for reception staff to find their records and brought them when they attended the centre. The centre's policy indicated that all patients' medical records were to be kept in the reception department. With doctors' permission, a number of patients, namely older patients and patients with chronic renal failure, were given their records to facilitate their access to be seen by the doctors. There were also many Syrian refugees visiting the centre to receive care which added more work pressure on the staff. A few patients brought two or more medical records when they came to see the doctors which resulted in a delay in the care provided for other patients:

A patient brought four files for his family members, and he asked the doctor to write medications prescription for all of them (Field notes). 
The doctor was unhappy about writing medication prescriptions for patients who did not attend the centre, so the doctor wrote a note in their records that the medication prescriptions would not be written in the next visit if the patients were not coming to the centre.

Data analysis indicated that HCWs such as the dentist, the receptionist, the pharmacist, the nurses and the laboratory technician had limited roles in providing health education to patients, which was mainly attributed to workload and work demands as reported by HCWs:

The pharmacist gives the medications to the patients without instructions for taking these medications because of work pressure or long waiting list of patients (Nurse. Interview 4).

Frankly, we don't deliver any education because we don't have time.... Load of work, work pressure and a long waiting list. Sometimes it is excellent if we take the medical history (Dentist. Interview 8).

These HCWs prioritised meeting the acute needs of patients due to time pressures, and health education was viewed as an optional extra if time permitted. Through observation at the pharmacy, I noted that many patients did not receive a brief education about how to take their medications. A few patients reported in the FGs that the pharmacists did not provide any instructions at the time of medication refills and their job was limited to dispensing medications:

Amer: Does the pharmacist explain to you about medications when you receive them?

Male patient 1: No.

Female patient 5: No (FG 3).

Many hypoglycaemic tablets are linked with meals such as Metformin (Unger, 2012). Pharmacists gave most medications in a small sealed plastic bag without providing patient information leaflets for each medication. Therefore, pharmacists missed an easy opportunity to increase patients' awareness about medications. It was apparent from the FG data that a few patients were not aware of the appropriate time to take their medication:

We need more education. For example, when they (the pharmacists) give us the medication for diabetes they tell me to take it 30 minutes before eating or during the process of eating or after eating. Sometimes, I forget or am confused (Female patient 5. FG 3).

A patient did not know how to take Insulin and Metformin. He was taking the medications 30 mins after eating (Field notes). 
Taking medications in the wrong way may limit the effectiveness of these medications. Lack of awareness as well as poor communication between patients and pharmacists contributed to the poor adherence to their medications. Poor centre design also hindered pharmacists in providing instructions about medications to patients.

During data collection, the pharmacist talked about her role in explaining to the patients about their medications:

Mostly ah mostly, but not all of the patients because most of them know and take their medications for a long time and so.... A few patients do not listen. Mostly, a patient who asks and shows interest I will explain to him (Interview 6).

In the above data extract, pharmacists presumed that patients were aware of the correct way of taking medications because they had taken them for a long time. However, patients may forget or take the medications in an inappropriate way or incorrect storage though they had taken the same medications for a long time. From the pharmacist's account, it seemed that if patients asked for information then it would be provided, but not all patients may feel comfortable doing that. However, the pharmacist was a source of health education, especially when the doctors were not able to provide it due to work demands. They only wrote /// an example to refer to a number of times that patients have to take medicine with little expansion of this role. Likewise, this extract highlights there was an expectation that the patient would ask questions and seek medical advice. However, patients in the Jordanian culture may be hesitant to ask questions for a number of reasons including respect for the HCWs, feeling silly or just not knowing what to ask. These reasons are linked with Parson's concept of the sick role (Giddens \& Griffiths, 2006) to explain why not all patients may feel able to ask the pharmacist for health information. In Parson's role model, the sick person must work to regain health by seeking medical treatment and following medical advice (Giddens \& Griffiths, 2006). Seeking advice is one of the patient's obligations to minimise the impact of illness in the sick role model. These findings are similar to another study conducted in Kuwait. Half of the 20 patients with Type- 2 diabetes who were interviewed mentioned that pharmacists have no role in medications education and their roles are only limited to dispensing medications without further instructions (JeraghAlhaddad et al., 2015).

This drug should be taken with eating or before eating, or in the morning. Ah, they do not know. Patients keep taking their medications in the same way since they started on the first 
day of their life. Then they come and tell us about having a stomach ache or problems in digestion or decrease in sugar (Pharmacist. Interview 6).

In the above data extract, the same pharmacist mentioned there was lack of awareness about taking medications among patients, and they were unsure how to take their medications even though she mentioned earlier that taking medications for a long time was one of the reasons for not educating patients. In Jordan, the focus on patient-centred pharmaceutical care is limited among pharmacists. Medication preparation and dispensing and inventory control are the main duties of most of the pharmacists in Jordan (Nazer \& Tuffaha, 2017). Therefore, the role of pharmacists in health education and promotion is not utilised effectively. In some hospitals in Jordan there are clinical pharmacists attending medical rounds and offering to counsel and documenting interventions (Nazer \& Tuffaha, 2017). The lack of the appropriate involvement of both nurses and pharmacists in the patient-centred team-based care increases the burden on the physicians and may lead to inadequate health education provided to patients with diabetes (Al-Keilani et al., 2017). Also, registered nurses demonstrated a knowledge deficit in clinical and theoretical-based topics, such as initial treatment of hypoglycaemia, insulin storage and preparation; meal planning and duration of action with hypoglycaemic agents in Jordan (Yacoub et al., 2014).

Lack of discussion with patients affected their participation in the management of diabetes. Though, one of the objectives of the National Strategy and Plan of Action against Diabetes in Jordan 2011-2012 was empowering patients (MOH, 2012). Patients may lose their active role in the long-term management of diabetes if they do not have enough time to communicate with HCWs. Through my observations in the nursing room, the average length of the patient's consultation with the nurse was 2-4 minutes. The nursing consultation focused on completing psychomotor tasks such as measuring blood pressure without actual communication with patients or at least asking them about their last blood glucose results which might limit patients' role in the management of their condition.

A few doctors narrowed their scope of practice and sharing knowledge with patients towards the written data in patients' records only. The following data extract is an example of dialogue reported by a patient whilst consulting with a doctor in the centre:

Same thing. Sometimes, I want to ask her (The doctor) a question and she replies 'my duty is to write the written medications and enough' I want to tell her about a special something 
happened with me she tells me 'according to what written for me, it is not permitted to give you something else' (Female patient 4. FG 8).

One of the essential elements of effective patient-HCWs interactions is encouraging patients to pose questions and to participate in the medical conversation, but in the above extract, there was a discouraging attitude held by the doctor. Another example reported by a patient who came to the centre on a busy clinic day:

Regarding the doctors in the centre, they are good. However, one time I came here at $1 \mathrm{pm}$ and I wanted to ask the doctor whom I used to trust her she replied to me 'you are number 60' I said I do not care even number 100 it was not my problem, so she got upset because I asked her (Male patient 2. FG 3).

This patient felt disappointed as he was not able to discuss another health issue related to his diabetes with the doctor. The doctor in the above quote told the patient that she had already provided care for 59 other patients, so she did not have time to discuss other health issues with the patient. The patient was unhappy with her response and told her that she had to listen to his other health concerns even if she saw 100 patients that day. As mentioned in the above data extract, work demands and staff shortages, especially of doctors, affected communication between patients and HCWs. The doctors were seeing on busy days 50 or 60 patients each, so they did not have enough time to encourage questioning from patients. Each doctor had to provide care for 30 to 50 patients with chronic conditions in an average clinic day (5 hours). Globally, the lack of primary care physicians and time constraint are barriers to effectively addressing the multiple complex problems presented by patients in primary care settings (Qidwai et al., 2011).

During my observations, I noticed that the family medicine clinics were very busy on a number of days and almost empty on other days. Sometimes, each clinic received 50 patients, and sometimes it received 15 patients. The total demands on health care services might vary considerably and at short notice according to factors such as the types and numbers of patients and days (Menzies, 1960). As I mentioned in chapter four, the dominant method of delivering nursing care is task-oriented which is when the total workload of a department is broken into lists of tasks, and each of these tasks is allocated to a nurse to perform (Zahran, 2011). The task-list system and task-focused care can make it difficult to adjust workloads when necessary by postponing or omitting less essential tasks (Menzies, 1960). Menzies' work (1960) aids understanding of what happens when there is a high workload and task allocation in that nurses 
are perceived as being very busy, they do not seek to engage with patients to elicit questions, and patients often do not ask the question as they can see the nurses are very busy. This also leads to each patient being cared for by a number of different staff which impedes the development of trusting relationships (Menzies, 1960). Providing only visible psychomotor care without engagement with the patients has been criticised (Menzies, 1960). The workload was also exacerbated by lack of an appointment system and lack of organisation to maintain a balanced number of patients during all weekdays:

The doctors said that clinics are busy on some days and they were empty on other days (Female patient 3. FG 6).

So patients who had their appointments during busy clinics days only received limited care, mainly medication prescription only. There was an appointment card given to patients and patients were only able to attend the centre on the day of the appointment or one day before or after their appointment. Patients also moved from long waiting queues to another as described earlier due to a shortage of HCWs. The main aspect of care provided during very busy clinic days was writing medication prescriptions as doctors had limited time to communicate with patients properly. Furthermore, there were essential requirements of patients that doctors had to complete in most consultations such as transferring the laboratory results to the patients' records, writing medications and request forms which resulted in patient dissatisfaction, incomplete patients' records and ineffective doctor-patient communication.

A patient's spouse indicated that patients might not receive a good quality of care due to staff shortages in the centre:

Yes, surely it is challenging. For example, the staff are not enough to provide care for patients.... The staff are not enough. For example, patients downstairs who are in the queue have to be served by two windows, one for women and one for men, but there is only one employee who serves people why not two persons there!!. Why there is a long queue (Spouse. Interview 3).

This family carer was disappointed and frustrated with the lack of staff. Therefore, the patients had to spend more time standing in the queue waiting for their records or their medications, especially when there were not enough HCWs to provide care for them. A few of the patients $(n=5)$ reported that many of the HCWs did not meet patients' expectations in terms of a physical examination and a male patient referred to the doctors as 'writers' because they only completed paperwork without actual clinical examination of patients: 
The doctor is a writer who only writes the prescriptions and doesn't ask you anything. Any employee can do the doctor's job which is writing prescriptions (Male patient 2. FG 2).

The term 'Writers' was used by patients as writing treatment based on verbal responses of the patients without a physical examination (Field notes). This occurred mainly on busy clinic days when there was limited time to spend with patients, so the doctors focused mainly on writing the medication prescription without considering other needs of the patients as shown in the following data extract:

The doctor should examine the patient EXAMINE the patient. For example, I told the doctor I got chest pain ..... Why do you have pain? Not paying attention or any feedback.....instead of measuring only blood pressure let the nurse take the heart beats because heartbeats are important for family medicine. I have visited this centre for years and for the first time I saw the ECG machine on my last visit (Female patient 3. FG 6).

A number of patients $(n=5)$ had only their feet and eyes tested once in the last five years:

We attended an educational programme about diabetes 4 or 5 years ago, and the doctor examined our foot. Since that time, I never have my foot examined, and I do not know about a diabetic foot. In the last five years, the doctor never had asked about other complications such as the diabetic foot (Male patient 1. FG 2).

Whilst during an observation period I noted that:

A patient who has diabetes for 15 years never went to test his eyes before, and he asked the doctor to refer him to the ophthalmologist. The doctor referred him to the ophthalmologist (Field notes).

In my study, a third of the patients $(n=11)$ suffered from diabetes-related complications. Patients may gain benefits when they are treated based on comprehensive care by considering the secondary impact of diabetes on other body systems. These findings are supported by others (Abu-Qamar \& Wilson, 2011; Albikawi \& Abuadas, 2015) who have reported that health care providers did not examine the feet of a person with diabetes on a regular basis in Jordan (AbuQamar \& Wilson, 2011; Albikawi \& Abuadas, 2015). Similar findings have been reported in Saudi Arabia and the United Arab Emirates (UAE) (Al Odhayani et al., 2017; Al-Kaabi et al., 2015). A fifth of the sample of 350 patients with diabetic foot disorder had never had their feet examined by health care providers in Saudi Arabia (Al Odhayani et al., 2017). Similarly, only 
$20 \%$ of 422 people with diabetes had received formal foot care sessions in the UAE (Al-Kaabi et al., 2015).

Incomplete medical records was another issue related to staff shortages and high workload as doctors were only able to document the basic notes of the patients' visit in their medical records. Lack of a patient's medical history, lack of test results, lack of order in writing notes, and not writing any changes in patients' medication profile are examples of issues related to incomplete medical records as observed by me and reported by participants. Incomplete medical records also affected patients' adherence to their management plan, continuity of care and follow up of the progress of patients:

A patient had stopped taking alternative medication without written notice in the file (Field notes).

A few patients have no laboratory results for 6 or 7 months in their records (Lab technician. Interview 5).

The nursing records contained data about the name of the patient, BMI, smoking history, BP, FBS, HbA1c and eye test. This information derived from seeing a blank nursing record. However, I did not observe the nurse writing or asking the patients about this data set. Another nurse told me during an informal conversation that a number of records only contained names of patients. During my observations in the nursing room, the average length of the patient visit was 2 minutes, and the average number of patients seen was 21 patients per 90 minutes. That means there was insufficient time to complete patients' medical records as required and to discuss patient concerns with patients.

Patients perceived that the visit to the centre was not as effective as there was a delay in the care provided for patients:

A number of patients spent more than 3 hours waiting to see the doctor then they only spent a few minutes with the doctor (Field notes).

We (HCWs) should see and sit with all the patients who visit the centre and give them enough time so the patient can talk about his problems. So that is the thing that I wish to happen to all patients here (Nurse. Interview 7).

This nurse wished to spend more time discussing patients' concerns but was constrained from doing so because of organisational factors. The doctors were also stressed due to a high 
workload over a number of days, so they went into focusing on completing basic tasks and not effectively communicating with patients. Menzies (1960), in her study of the nature and effect of the anxiety level in the hospital setting identified that the nurses who provided care for a large number of patients were only able to perform a few tasks and had restricted contact with patients. The nurses were forced to focus only on performing the important tasks and to reduce the less important ones, which were considered talking with patients due to the pressure of work (Menzies, 1960). Nurses were not eliciting conversations with patients according to Menzies as a self-preservation tool to enable them to manage the high workload and associated stress (Menzies, 1960). Therefore, the time factor and the number of patients needed to be seen affected communication between the patient and the nurse, and between the patient and the doctor. As a result of the high workload, patients did not have the opportunity to express their needs the nurses.

The HCWs in this study were not acting as a multidisciplinary team:

The diabetes nurse came to the nursing room and she asked other nurses to refer patients with diabetes to her for health education, but they did not refer any patients though there were patients with high BG (Field notes).

During my observations, I observed there was a lack of coordination regarding health education among HCWs and health education was mainly provided by the doctors, the diabetes nurse and the dietitian in the centre. The dietitian and the diabetes nurse were available on most days, and their role was to educate patients about diabetes. The doctors and nurses who managed patients with diabetes had to refer patients to the dietitian and the diabetes nurse for health education. However, only a few patients were observed as being referred to receive health education due to lack of coordination and workload. Six patients in the FGs mentioned that they had been asked by doctors to see the dietitian and diabetes nurse.

Providing patients with written information was not well used during observed consultations. A physician presumed that written communication was an ineffective mode of communication:

We don't have posters, and nobody reads in this centre. If you give the patient brochures, he will not read, and it will be discarded in the dustbin later (Physician, Interview 1).

In addition, not all nurses valued written information as aiding health education:

Patients do not read the provided brochures by the Ministry of Health (Nurse. Interview 2). 
Half of the HCWs $(n=5)$ interviewed perceived that patients did not read and they preferred to receive auditory information rather than visual information. During 60 hours of observations, only one doctor asked two patients in different consultations to write down the normal values of blood glucose levels and the right times of taking medications. These patients showed cooperation by writing the instructions provided by the doctor, and they reported feeling satisfied at becoming more involved in their care.

The use of intimidation to change health behaviours of patients was proposed by approximately two-thirds of the HCWs ( $n=7)$ interviewed:

When we (HCWs) tell patients about diabetes complications, so they are afraid of having it. A few patients had shown interest when they saw other patients with diabetes complications. We asked health care providers from the National Diabetes Centre for talking about diabetic foot and showing pictures of diabetes complications. Patients showed interest because they were afraid of the development of complications (Dietitian. Interview 3).

I intimidate (make patients feel fearful) by telling them about complications such as numbness in extremities, vision problems, diabetic neuropathy and a decrease in sexual performance (Nurse. Interview 2).

The nurse used 'fear-based messages' to alert patients to the possibility of developing long term complications. The aim of the 'fear-based messages' was to encourage patients to perceive that diabetes is a serious condition and the consequences of poorly controlled diabetes are significant enough to adhere to the management plan.

Intimidation and fear-based messages were part of HCWs-patient communication and fear of development of diabetes complications was the main reason for introducing intimidation. The aim of using intimidation was to encourage patients to engage in healthy behaviours and to change their daily habits. The use of intimidation aimed to prevent long-term complications associated with diabetes. Showing pictures of diabetes complication was welcomed by a number of patients as well as family carers:

People with diabetes in Jordan follow the recommended regimen when they see or have the complications. For instance, showing pictures of diabetic foot and complications may lead patients to follow the treatment regimen (Male patient 1. FG 2).

Whilst a family carer commented: 
People will learn a lot when they see pictures of patients who lost their legs due to diabetes. Patients will be afraid to lose their limbs, so intimidation is better (Son. Interview 1).

A number of patients and family carers $(n=5)$ supported the use of intimidation as a means of communication by showing pictures of diabetes complications as a preventive method to motivate and encourage adherence to diabetes management plan. This family carer perceived intimidation by showing diabetes complications as cues to action that stimulate and trigger the patients to take action and change their habits. In the HBM, patients with a high perceived threat are more likely to engage in healthy activities and change their behaviours (Becker et al., 1978).

A physician and the supervisor of Health Promotion did not introduce intimidation as a method for patients with diabetes from the beginning. Instead, they explained the benefits of good glycaemic control level:

We explain to patients what benefits will happen if they control their blood glucose. This process consists of 4 stages which are an explanation, intimidation, commitment and controlled diabetes (Physician. Interview 1).

'Temptation and intimidation' topic is good for patients with diabetes when you explain to them what benefits they will have if they control their blood glucose level. If this does not work, we will show them bad pictures of previous people who didn't follow the recommendations so they may get afraid of that (Supervisor of Health Promotion, Interview $10)$.

This doctor talked about a four-stage process that he followed while providing health education for patients with diabetes, although I could not find any evidence base to support this method in the literature. This method was used in the centre by the doctors mainly to encourage patients to change their behaviours. However, approximately a third of patients $(n=11)$ in my sample had already developed complications such as diabetic foot, neuropathy, oral health problems and nephropathy after being diagnosed with the condition.

Approximately more than half of the patients $(n=22)$ had been diagnosed with diabetes for more than five years, which meant that a number of patients had developed diabetes complications within a relatively short period, although it is acknowledged that patients often received a diagnosis of diabetes many years after its first onset due to delays in seeking medical advice. In addition to personal factors, many factors contributed to poorly controlled diabetes 
including organisational and the influence of health policies which have been reported in this chapter. It would seem, therefore, that using intimidation by focusing only on the complications of diabetes may be not effective in preventing or at least delaying the development of complications. For instance, based on the chronic conditions care indicators in the centre derived from chronic conditions datasheet, $55 \%(n=162)$ of 295 patients with diabetes had their HbA1c greater than 7\% between January and August 2016.

These patients were more likely to develop diabetes complications due to their high blood glucose levels. The HCWs could usefully review their health education approach instead of focusing primarily on the use of intimidation. Patients may feel stressed because they may fear developing complications.

Two family carers, three patients and HCWs such as physicians, dietitian and the supervisor of Health Promotion encouraged the use of intimidation as an effective method of preventing complication. Eleven patients had already developed complications. A few patients felt dissatisfied about the use of intimidation due to feeling stressed.

The findings in this chapter indicate influence at the interpersonal level, organisational and policy level on patients' adherence to their diabetes management plan. The socio-ecological health model (McLeroy et al., 1988) was useful for aiding understanding of the factors which influenced patients' experiences of Type-2 diabetes at the organisational and policy level. In this chapter, patients, their carers and HCWs reported challenges that went beyond a focus at an individual level by recognising challenges related to national health policy and the local health care centre. McLeroy et al. (1988) mention that an individual's health is affected by national health policies, national health services and regulations, and any changes in the national policies imply changes in the patient's treatment programme. Therefore, the SEM (McLeroy et al., 1988) suggests the calls for multilevel interventions that incorporate social, institutional and policy approaches to improve patients' health.

\subsection{Summary}

This chapter has discussed the main challenges of diabetes management which were identified and grouped into two main levels: the health care system level and the health care workers level. Patients perceived that medication shortages and changes in medication brands affected adherence to their medication regimen. HCWs reported that the ability of patients to visit more than one health care facility resulted in patients' confusion and hoarding of their medications. Finally, the findings of this study reported that workload pressures, compounded by an 
inefficient appointment system and staff resulted in limited and ineffective communication with patients which negatively influenced patients' adherence to the management plan.

The following chapter presents and discusses facilitators to good glycaemic control levels reported from the perspectives of patients, their carers and HCWs. 


\section{CHAPTER EIGHT}

\section{FACILITATORS TO GOOD GLYCAEMIC CONTROL LEVELS}

\subsection{Introduction}

This chapter presents and discusses the subthemes and categories related to the facilitators as suggested by participants to improve blood glucose levels among patients. As most of the patients, HCWs and family members reported barriers and challenges that hindered patients' adherence to their management plan, they also reported facilitators to improve management of diabetes or to limit these barriers. Participants reported a number of wide-ranging suggestions that may improve management of diabetes at two main levels of care: at the local comprehensive health care centre and the national health care system.

In the following subtheme, I discuss the main findings related to strategies to improve diabetes management at the comprehensive health care centre level.

\subsection{Facilitators at the comprehensive health care centre level}

Within this subtheme, four main categories are presented: the role of health education, the doctor for herself/himself, sources of health information that raised patients' awareness about diabetes and continuity of care.

Firstly, I present and discuss the role of health education as suggested by most of the patients, HCWs and patients' family carers as a facilitator to help change patients' health behaviours and improve their blood glucose levels.

\subsubsection{The role of health education}

In this category, I discuss the findings regarding the previous educational programme provided at the health centre which some of the patients in the focus groups had attended, the development of an educational package for patients based on their needs, and formats suggested for future health education programme by patients, HCWs and patients' family carers.

The previous health education programme and materials are reviewed now.

8.2.1.1 Overview of the previous health education programme at the comprehensive health care centre. 
Approximately a third of the patients $(n=14)$ had attended formal health education in the last year and reported that these programmes were the main source of health information. The collected data about the previous programmes were collected through discussion with the dietitian, the nurse who had been involved in delivering the programme and from patients through the focus groups. Also, I participated in a similar programme in another centre as this was a national programme with a set syllabus and reviewed the materials.

The previous health education programmes were part of an international project called 'Global Micro-Clinic Project' (Micro Clinic International, 2017). Three projects were conducted by Micro Clinic International in the centre between 2010 and 2016. These projects were conducted in the centre in conjunction with the Ministry of Health (MOH) and The Royal Health Awareness Society (TRHAS). Each project consisted of four health education programmes per year. This was a six week programme usually as one lecture per week.

The participants were recruited for the programmes by two methods: through doctors by referring the potential participants to the dietitian or when the patients visited the dietitian (Dietitian. Interview 3).

The dietitian contacted the participants one day before the scheduled session to confirm attendance and reminded them of the time and venue of the session. Meals, lab tests, health club sessions and small gifts were examples of incentives provided in the first two projects to the participants:

During the health programmes, we took the patients to a health club, and there was a trainer and patients worked out by using cardio machines and dumbbells (Nurse. Interview 4).

There was social interaction between participants and they established a good rapport with other participants (patients and family carers). The nurse and dietitian encouraged the participants to bring their family members or friends to the courses. More female participants attended the courses than males. The courses were delivered by the dietitian and nurses who had to complete six days of an instructor's course:

This programme is supported by the Royal Health Awareness Society, and the programme is available in multiple centres. They train a nurse and a dietitian to teach these programmes. The lecture is every week or two weeks, and they use data show to teach the patients (Supervisor of Health Promotion. Interview 10). 
The potential participants were people with diabetes, people who were at risk of developing diabetes and their family carers. Each 'Micro Clinic' consisted of a small group of participants (three to five individuals) such as two patients and their family carers which was also considered as a social activity session. The participants were unable to attend more than one course in the first two projects. In the last project, the participants were able to attend multiple courses. The first two projects were financially supported, so the average number of attendees was 25 to 30 participants, but when the financial support ceased in 2015, the number dropped to five participants:

Lack of incentives and external funding support were the reasons behind a small number of participants attending the last programmes and stopping these projects in the selected centre (Dietitian. Interview 3).

The average number of attendees in the first two projects was 1500 participants over two years in the studied centre, and this number dropped sharply in the last project. In the last project, two or three participants completed all sessions in each cycle with the cost and lack of incentives being key barriers to participation.

Though these programmes had stopped in the centre more than six months before I conducted my study, patients' responses showed the benefits of the educational programme, and they had still retained a lot of information from the programme:

I attended an educational programme at this centre, and the information was easy and useful. I am still learning about diabetes (Male Patient 1. FG 2).

The centre here conducted educational programmes for patients with diabetes, and they conducted many courses. These programmes increased my awareness about diabetes (Female patient 1. FG 7).

As they told us previously in the educational programmes, damage inability of the pancreas in secretion which results in instability in the human body because of diabetes. Low blood sugar leads to shivering, hunger and sweating and high blood sugar leads to frequent urination and numbness in the body extremities (Male patient 5. FG 4).

The supervisor of Health Promotion also attributed stopping these programmes in the centre as health promotion is not perceived as a priority by the HCWs and the health care system: 
The first element of primary health care is health education then medicine and the medications is the last element. Although the most important element is health education, there is no funding support for it, for that it stopped. So it is not important to talk to the patient, a patient takes his medications and leaves. However, health education is not important for some HCWs which means it is not important. For example, the doctor prefers to treat patients and sits in the clinic more than to go outside for conducting the lecture and educating patients (Supervisor of Health Promotion. Interview 10).

This supervisor perceived that health education is the most important element of primary health care; therefore it should be a priority. She reported that a group of HCWs, primarily the doctors, did not have active roles in conducting health education sessions in the health centres. Her responses showed how the medical model informed doctors' perception by focusing more on cure and medications than lifestyle and behaviour change, which can be initiated by health education (Wills, 2014). Regarding the HCWs, half of them $(n=5)$, a physician, the supervisor of Health Promotion, a dietitian and two nurses, of those I interviewed attended formal educational training about diabetes though all $\mathrm{HCW}$ s mentioned that they provided direct care to patients with diabetes.

I visited another centre, $5 \mathrm{~km}$ away from my centre, where the programme was still running and participated in the educational programme, taking notes about the information given and observing the style in which the information was delivered. I attended two sessions and six attendees were there. A nurse and a dietitian delivered the sessions. Weight measurement and a diet plan were the incentives for the attendees. Health education sessions had been conducted at this other centre since January 2016 at the same time these programmes were stopped in the centre where I conduct my study. This implied that the 'Global Micro-Clinic Project' had shifted from one centre to another without achieving sustainable health education programmes. Zoughbie, Watson, Bui, Farraj, Prescott and Ding (2014) conducted a study to find out the effectiveness of the Micro Clinic health educational programmes in achieving long-term health improvement among 315 patients with diabetes or pre-diabetes in Jordan. Data collected included $\mathrm{HbA1c}$, BMI and weight measurements at the starting day and the end of the four month programme, 12 months and 24 months after completion of the programme. 90 participants were lost to follow up over the 24-month period. The study findings showed that participants had sustained the long-term reduction in weight (on average $1.8 \mathrm{Kg}$ per person) 
and $\mathrm{HbA} 1 \mathrm{c}$ (on average $0.46 \%$ ) which indicates the benefits of health education in facilitating behaviour change.

The educational documents available for review in my study included the previous diabetes education programme that consisted of eight sessions, attendance registers, one objective sheet and four educational video clips. There was a weekly weight measurement at the beginning of each session and participants were asked to write down their weights. Participants were also asked to do one physical movement at the end of each session. The previous educational programmes in the selected centre emphasised the key role of participants to raise awareness in their local community by giving all participants who completed the programme the title of 'ambassadors of the Micro Clinic programme in the society'. This implied that participants were taught to be diabetes champions to raise diabetes awareness among their family and friends, similar to the Diabetes UK Community Champions (Diabetes UK, 2018a). My focus group discussions provided a good example of how patients shared and exchanged knowledge with each other that they had learnt in the previous educational programmes. The programme also emphasised the importance of diabetes management behaviours (diet, physical activity, SMBG and medications) in each session to achieve successful diabetes management.

Each participant received an objectives sheet that contained six nutritional and six physical activity objectives as a workbook to monitor their weekly progress. Participants were encouraged by the educators to achieve one nutritional goal and one physical activity goal every week. At the end of each session, participants were asked to complete the achieved objectives. The following is an example of a weekly goal that participants were asked to complete in the previous educational programme:

- Practice resistance training _ times this week for _ minutes. For instance, stretch band exercises, push-ups, sit-ups and squats or weights:

$$
2 \text { times, } 10 \text { minutes } \text { or } 3 \text { times, } 10 \text { minutes } \quad \text { or } 3 \text { times, } 15 \text { minutes }
$$

The programme consisted of eight sessions; the first session was an introduction to the programme and diabetes: types, risk factors, signs and symptoms, prevention and management. The second session discussed the dietary requirements for patients with diabetes, food portions and food groups. In this session, the programme emphasised the differences and compared a diabetic diet and a normal diet. As reported in chapter six, patients perceived that a diabetic diet is a complex dietary modification that they were not able to follow or to incorporate into their family meals. Therefore, the previous educational programmes contributed to these 
patients' perceptions that following a recommended diet is difficult to achieve. It was a missed opportunity in the previous programmes to encourage patients and their family carers to eat the same appropriate healthy diet that also meets the needs of patients with Type-2 diabetes. By the whole family eating similar healthy meals, this would also help to alleviate tensions in family relationships and facilitate adherence to the dietary recommendations.

The third session discussed steps to initiate physical activity, walking with a partner, considerations before, during and after walking such as testing BG, wearing the right shoes and muscle stretching. The programme placed more importance on walking than musclestrengthening exercises. Walking was the dominant outdoor form of exercise among patients in this study. However, as previously stated, walking alone is not sufficient to meet WHO recommended physical activity levels. The recommended physical activity guidelines for adults are 30 minutes of moderate to vigorous intensity exercise per day on five days and muscle-strengthening exercises on two or more days a week (WHO, 2011).

The fourth session focused on the importance of regular check-ups, examples from daily life activities, low and high BG levels and prevention of complications. The fifth session discussed types of medications, the mechanism of medications in the body, how to remember to take medications and rotating insulin injection sites.

The sixth and seventh sessions focused on diabetes complications, heart problems and prevention of complications. The last session included a closing ceremony and a review of the main objectives of the programme. By the end of the programme, every participant who completed all sessions received a hard copy of the educational materials provided by the Ministry of Health.

The educational materials were provided by TRHAS and it was translated from English to Arabic. The International Diabetes Federation (IDF) documents were the main source that was used in developing the educational material. The problem with translation is that content may not always deliver accurate meaning. For example, the word medications was translated to treatment in Arabic (علاج) in the educational material which may have influenced patients' perceptions that medications can cure as presented in chapter five. During data collection, I noticed that most HCWs and patients used the term treatment to refer to medications. Therefore, the patient or family carer may think that medications are the only treatment for diabetes. My study findings suggested that using the actual Arabic term of medications (الأدوية) among HCWs instead of treatment may help to rectify patients' misconceptions of medications. 
There was a cartoon show of 4 episodes that were used to educate participants in the educational programmes previously provided in the centre:

I like using the TV for health education. Before there was a TV series about diabetes and people liked it...... TV series contain information about diabetes, types and complications. This TV show provided general information about diabetes (Nurse. Interview 4).

This cartoon show of 4 episodes used simple and easy language to educate patients about diabetes. This TV show was called 'Abu Sami' (father of Sami), and it showed different daily life activities of a patient with diabetes such as eating and exercising (Figure 7).

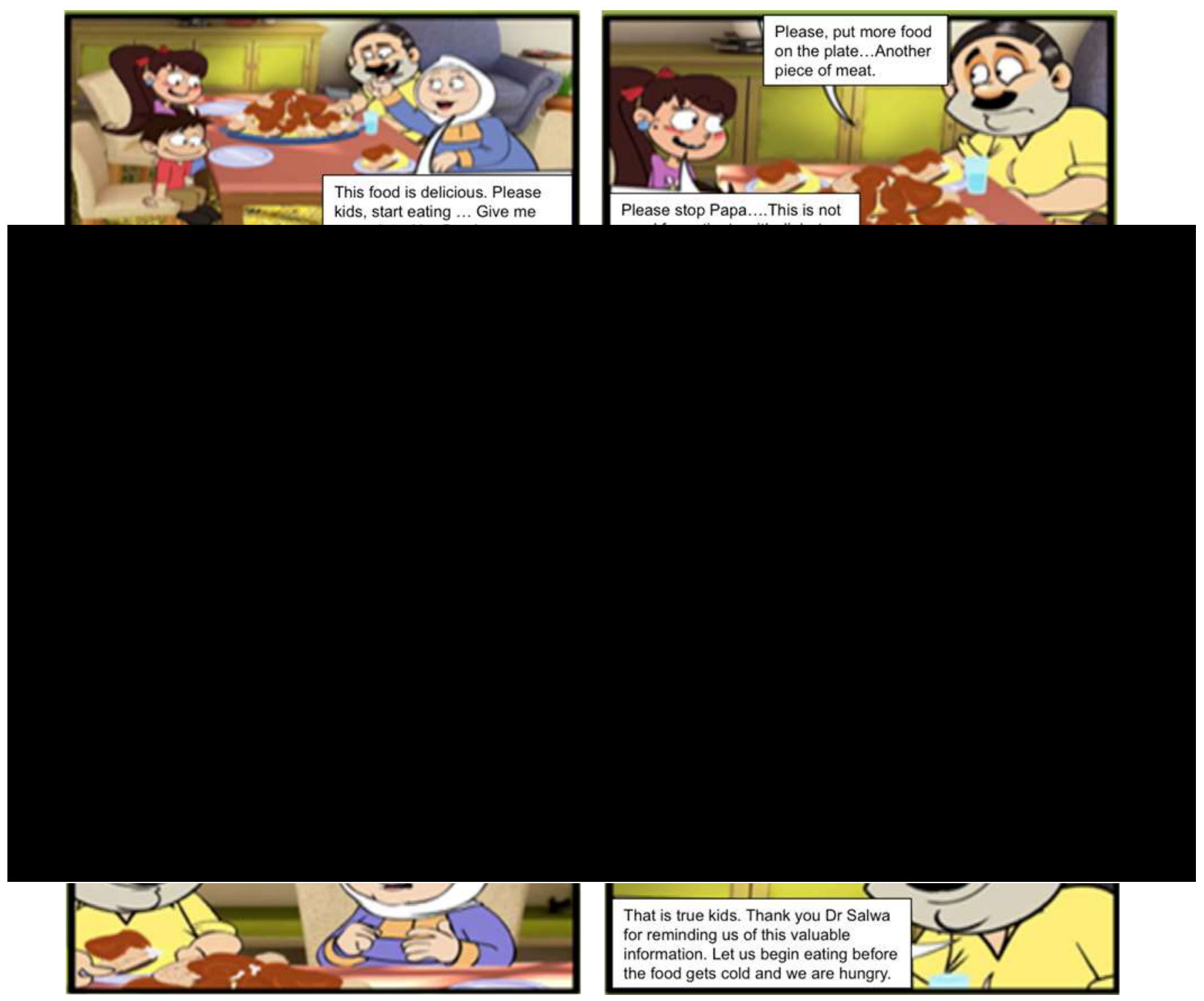


Figure 7 shows pictures and the English translation of the dialogue between Abu Sami and his family taken from the educational show 'Abu Sami' (adapted from http://microclinics.org/projects/middle-east/jordan/)

In this section I have provided the reader with information about the previous health education programme and provided a critique of it. Overall, participants were satisfied with the previous educational programme. However, patients, HCWs and family carers suggested topics to inform the development of an educational package for patients with Type- 2 diabetes. These topics are discussed now.

\subsubsection{Future health education programme}

Participants provided information on the topics that they thought needed to be covered in any future health education sessions:

Awareness about diabetes-related complications and its symptoms (Male patient 4. FG 6).

Complications. What are the complications in the future? Such as damage to retina or damage of legs or breakdown we can avoid them as possible (Male patient 5. FG 8).

Learning about diabetes complications was the most common topic reported by many participants though there were two sessions about complications in the previous educational programme. This may be related to over a third of the patients in my sample had already developed complications which may induce them to learn more about their complications.

A few patients $(n=3)$ also suggested that stressful factors should be covered within any future educational programme:

I like to learn about the life situations that may upset me, and I keep them away (Female patient 4. FG 8).

Frankly, stressful factors and why is the pancreas damaged completely by bad psychological status? As I have become taking insulin because of stress (Female patient 3. FG 3).

Patients may be enthusiastic to learn about stress factors due to over a third of patients who participated in my study believing that having psychological distress was the main cause of diabetes. 
A physician suggested that the emotional component of food should be covered in a future educational programme:

Cultural factors should also be included such as patients feel deprived or deprivation phobia (Physician. Interview 1).

In chapter six, food was perceived as a sensitive issue for many patients. Food was more than just calories, and these patients were emotionally attached to food by expressing feelings of discrimination and deprivation. A few patients $(n=2)$ suggested that a discussion about herbs should be included in future health education programmes:

We want to learn if herbs are scientifically proven (Male patient 1. FG 1).

I like to learn about herbs (Male patient 3. FG 2).

In chapter six, approximately half of the patients $(n=18)$ reported that they used herbs and there were variations in responses among patients regarding the advantages of using herbs to aid control of their diabetes. In comparison to the topics in the previous educational programmes, the emotional component of food, stress and its management, and the properties of herbs were not covered in the previous sessions. The previous health education sessions placed more focus on the physiological effects of diabetes than other dimensions of health such as spiritual and psychological aspects which is consistent with the medical model of practice (Giddens \& Griffiths, 2006).

Many patients also suggested that diabetes management behaviours (diet, physical activity, SMBG and medications) and food portions be covered in a future programme:

There is another issue which is the topic of portions. For example, eat three portions of fruits daily. What are the portions? Is the bunch of grapes portion? Or is one apple portion? What are the portions when we say it? (Male patient 3. FG 5).

Summary of how to take medications, diet and sport (Male patient 6. FG 4).

The previous programme placed more importance on these topics as they were covered in four sessions, but it seemed that the patients in my sample wanted to know how to apply this knowledge to their everyday lives.

One male patient had a different perception regarding designing health education sessions. He suggested that the educational programme needed to be individualised and designed by the doctor based on individual needs: 
I think the programme should be designed by the specialist doctor because the doctor knows the patient's situation, his life circumstances, his blood sugar, his diet and his drink (Male patient 2. FG 5).

This patient perceived that the doctors were more aware of a patient's health care needs. Therefore, they were more able to address the areas that had to be covered in the health education programme. This 53 years old male patient, secondary school educated, had been diagnosed with diabetes for more than ten years. His responses during focus group discussion showed that he had gained a lot of information about diabetes by attending educational programmes. He also mentioned that he had attended a previous formal education programme in the centre. This patient's case was a deviant case that refuted the general perception of all patients about their role in designing health education sessions. Deviant or negative cases are a group of episodes that refute the general supposition regarding the studied phenomenon (Morse \& Field, 1996). These cases are atypical of the representative cases and including them in data analysis provides another insight (Morse \& Field, 1996) and further exploration of reasons behind poorly controlled Type- 2 diabetes. The education sessions such as group discussions would complement and support one to one consultation with patients.

However, during my observations, health education was only delivered informally by the one to one method when the patients visited the doctor to collect their medications. Therefore, health education was delivered opportunistically with no scheduled sessions or topics scheduled in advance with sufficient time allocated for the sessions. In previous chapters, due to time pressures, doctors did not routinely offer health education or refer to other HCW for this information to be provided and mainly only responded to questions posed by patients. This leads to inequity in that some patients often those who may have a little more knowledge receiving further information and others receiving none.

From patients' and doctors' accounts, they were referring to health education as opposed to wider policies changes which constitute health promotion. Health education has been defined as any combination of learning experiences designed to enable individuals and communities to enhance their health, by increasing their knowledge or influencing their attitudes (WHO, 2018a). While health promotion is defined as a process of enabling individuals to take control over and improve their health which goes beyond a focus at an individual level by recognising social and environmental interventions (WHO, 2018b). 
In addition to the suggested topics to develop a future educational package, participants reported their preferred methods for delivering health education in the future. These methods are discussed now.

\subsubsection{Preferred formats for future health education programmes}

During FG discussions, all patients were asked about the format of health education session which they felt would be most appropriate for them. Approximately half of the patients $(n=16)$ preferred receiving health education by attending a focus group or group discussion over other sorts of formats:

Yes, group discussion which we open our hearts and talk frankly (Male patient 2. FG 1).

Group discussion as few patients should participate in the discussion so that everyone will gain benefits either from the health care professional or the participants (Female patient 3. FG 6).

Group discussion encouraged this male patient to share and reveal his concerns and feelings with other patients. He felt socially and emotionally satisfied by discussing his concerns with other patients. Female patient 3 indicated that patients learn from other patients' experiences and exchange information. An example of the focus group being a learning opportunity for patients was a discussion among two male patients about starchy foods in focus group 5:

Male patient 3: My spouse and I don't know what we have to eat. How rice increases blood glucose, it is unbelievable!!!!! So these things that I should know. It is believable!!! That rice and bread increase blood glucose.

Male patient 2: This brother asked what the link between starchy food and sugar is. There is a link. There are fruits sugar, table sugar, potato sugar and starchy food. All contain sugar and are converted to sugar in the body.

The above discussion showed that the male patient 3 was not aware of the composition of common foods and how to eat appropriately to maintain good glycaemic control. He did not know that all sources of carbohydrates are broken into simple sugars in the human body which in turn elevates BG levels. Male patient 2 disagreed with him, and he showed a better understanding of the management of diabetes and explained it in simple terms which the other participants seemed to understand. This group discussion provided a good opportunity for 
patients to speak and listen to each other, exchange knowledge and help each other. My group discussions also identified incorrect information and misconceptions (Section 5.5) that were shared by a group of patients:

Type-2 diabetes usually occurs in old people and rarely with young people (Male patient 4. FG 6).

People told me that honey is mixed with other ingredients will decrease the symptoms of diabetes, and somebody used it (Female patient 4. FG 7).

As a qualified nurse and researcher, this incorrect information and misconceptions were addressed during debriefing sessions after each focus group discussion. Another example of the advantages of conducting focus groups or group discussions was regarding seeking information. Patients showed a desire for seeking more information about the management of their condition. They were given the opportunity to ask questions related to their condition during the discussion. All focus groups were conducted by me, aided by a female assistant (dietitian) who made female patients more comfortable during the group discussions. A debriefing session was conducted after each focus group by me in the presence of the female assistant (dietitian) to answer any questions related to diet. Therefore, patients had the opportunity to express their understanding of the topics discussed and to ask more questions:

If you give us advice. We want to ask you whether the herbs are good or not? (Male patient 3. FG 7).

What is the normal HbAlc value? (Female patient 1. FG 8).

In addition to my research purposes, the focus group or group discussion format was reported by patients as a health education session and as an effective tool to increase their awareness about their condition. They enjoyed participating in a group discussion:

We enjoy talking to these nice people. When there is a group discussion, the person may gain benefits, and he will gain benefits from others' views. Now we are 5 or 6 persons, everyone may develop an idea, so there is developed ideas but when he is alone (Male patient 5. FG 8).

A lab technician suggested that a group discussion would be the recommended method of health education because of the patients' age group: 
I think it depends on the target group. Usually, they are old patients, so the group discussion is the best. You know people in this age group don't work and they stay at home. So when you ask them to gather in one public place for health education, patients will gather and talk about their problems. When the patients speak to each other, they feel relieved, so I think the group discussion is the best (Lab technician. Interview 5).

This lab technician suggested that older patients preferred group discussion in comparison to other age groups. She reported that older patients are more likely to speak freely within the same age group. Age and educational level could be other factors that explain why patients preferred to attend the focus group. Two-thirds of the patients were aged over 50 years $(n=25)$, and approximately two-thirds $(\mathrm{n}=23)$ of patients had completed secondary school. These findings suggested that age and educational level need to be considered when designing a health education session. Sociodemographic factors such as age and educational and reading levels of patients should be considered in developing and tailoring of educational materials (Glanz, 2002). Although the evidence from my study revealed that approximately half of the patients $(n=16)$ preferred group discussion, the remainder mentioned different formats that are discussed later in this section. I was unable to locate any previous studies that show that group discussion is the best method for patients who are aged over 50 years. However, there is a need to develop health education sessions with the target audience in mind. Assessing the needs and priorities of the target audience is essential to promote the adoption of a behaviour that will improve the health or well-being of the target audience (Huff et al., 2015). The target audience is classified by distinct characteristics and needs such as disease and age (Huff et al., 2015). This lab technician also perceived that a group discussion is similar to older patients' daily activities where they gather and talk to others. Group discussion is a more naturalistic method that draws on people's daily living activities and experiences of talking and expressing themselves with others (Wilkinson, 1999).

Approximately half of HCWs ( $n=5$ ) suggested that a group discussion is the best method of delivering health education. However, through my observations, I noted that health education was only delivered by the individual, face to face method when the patients visited the centre on a monthly basis. This implied that some patients might receive overwhelming information in one short single session and others may receive no information as written information in the form of leaflets was not provided. My observations also showed that health education is primarily delivered informally in the form of a discussion between the doctor and the patient during the visit which on average lasted five minutes: 
As the session is not scheduled, some barriers face the patient to receive health education during his/her visit to the centre such as long waiting list of patients, unavailability of the dietitian or the doctor and lack of time (Field notes).

In addition to the group discussion, 22 patients reported a range of different formats to deliver health education such as audio-visual, face to face and lectures or combining multiple methods:

Lectures. The person who comes especially to attend the lecture wants to learn and understand about diabetes. I prefer if they conduct lectures from time to time. I think the lecture is the best thing (Male patient 2. FG 5).

Multiple methods. I always prefer if there is a practical method in front of patients, so they like to come and attend. Always, if you talk and patient listens he will be bored. So every time with the different method and if you do something in front of the patient, he will like to attend and receive the information (Female patient 4. FG 3).

This male patient perceived that patients who attended lectures are self-motivated to seek health education. Multiple methods also give patients the opportunity to engage in, for example, physical activity. Female patients $(\mathrm{n}=12)$ were more likely to prefer focus groups than males $(n=5)$. There was no gender difference in preference for other reported formats.

In this category, I have discussed the role of health education to promote the patients' management of their condition. In the next category, I discuss how patients and a few HCWs perceived that health education would empower patients.

\subsubsection{Doctor for herself/himself}

Patients who attended previous educational programmes showed more awareness regarding their management of diabetes. Approximately a quarter of the patients $(n=9)$ out of 38 patients also believed that health education to be an effective tool in empowering them to take control over their own condition. 'Doctor for herself/himself' is a common phrase used by a number of participants to refer to patients who become experts on their condition by knowing their health needs, understanding their condition and taking more control over their own health. For example, when a patient experiences symptoms of hypoglycaemia, she/he will have a sugary drink and test his/her blood glucose without seeking immediate medical help unless if needed. Approximately one-sixth $(n=5)$ of the patients, a receptionist and the supervisor of Health Promotion used the term 'doctor for himself or herself' to describe their views on the benefits 
and values of health education. This implied that through health education patients were able to empower themselves and to become experts on their condition:

Health education. The lecture is necessary, and education is very useful too. When the patient is more educated, he will be more aware because he is a doctor for himself eventually. The doctor or the medical staff will not be with you all the life. The patient should be educated, and it is necessary to know everything about diabetes (Male patient 2. FG 5).

It is health education. The doctor should spend more time with the patient and educate him about management plan to the level that the patient will be to look after himself and to be a doctor for himself. This disease is a chronic disease, and the patient should live with it. The patient should be a doctor for himself, but he needs supporters such as the community, health centre and family. Family members support him, reminding him of medications' time, walking, stopping smoking and losing weight because he should not be responsible for himself alone (Supervisor of Health Promotion. Interview 10).

Male patient 2 suggested that health education will enable patients to understand their condition better than other people do. Patients gained a lot of knowledge and experience because of living with diabetes for a long time. This perception included that patients will not only receive the medical instructions, but also will be involved in any decisions regarding management of their condition. The supervisor of Health Promotion also suggested that the management of diabetes can be shifted to give more responsibility to the patients. However, she perceived that doctors need to ensure that the level of patient's awareness is adequate to enable effective selfmanagement which could be achieved by appropriate health education and through increasing the length of the patient's consultation with the doctor. She also reported that patients could not be empowered without partnership and shared responsibilities with the surrounding community, the health care system and HCWs. This partnership can facilitate the process of patient empowerment and behavioural change by removing barriers and obstacles that are beyond patients' control and through offering support when patients seek to make behavioural changes. These views are more consistent with the concept of health promotion and are consistent with the essential components of the process of patient empowerment as proposed by the World Health Organisation (WHO) which are: patient participation, knowledge, skills and presence of a facilitating environment (WHO, 2009b). The WHO (2009b: 190) defines empowerment as 'a process in which patients understand their role, are given the knowledge and skills by their health care provider to perform the task in an environment that recognises 
community and cultural differences, and encourages patient participation'. Although the previous health education programme gave patients knowledge and skills, but did not address patients' role, take into account cultural differences or encourage participation.

The previous health education sessions in the centre provided a group of patients with essential knowledge and skills to manage their condition effectively and the confidence to share their knowledge in the group discussions which is considered as an example of patient empowerment:

The training course gave us background information about diabetes to change wrong behaviours. They told us do not inject insulin in one place and draw a circle because injecting insulin in one place may lead to damage to skin cells (Male patient 1. FG 2).

Male patient 1 reported that increasing knowledge of patients through health education would lead to behaviour change and to develop necessary skills that enable patients to take control over their own health. These responses are consistent with the educational approach (Naidoo $\&$ Wills, 2009) that aims to provide information to enable patients to make decisions about their health behaviours. This will be achieved by conducting health education programmes or providing educational material. Several studies have shown that diabetes knowledge is important to change management behaviours among patients with diabetes in Jordan (AlSarihin et al., 2012, Al-Khawaldeh et al., 2012, Adwan \& Najjar, 2013; El-Qudah, 2016). However, knowledge alone is not enough to change patients' behaviours, and it should be complemented by behaviours and positive attitudes towards their own health (Naidoo \& Wills 2009).

In the United Kingdom, patients and HCWs can attend X-PERT health training programmes to be trained educators (X-PERT health, 2018). This implies that patients are empowered and supported through health education to build confidence and to be equal partners with HCWs in the management of their condition.

One of the objectives of the National Strategy and Plan of Action against Diabetes in Jordan 2011-2012 (p. 12) was "empowering patients to care for themselves" through health education for them and their family carers $(\mathrm{MOH}, 2012)$. Self-monitoring blood glucose (SMBG) was reported in this strategy as a target objective to promote patient empowerment. Patients may enhance their active role in the long-term management of diabetes when they are asked by HCWs for their views in developing the management plan. Therefore, patients may take a more active role and responsibility to control their own health, which in turn may increase their 
internal locus of control (Rotter, 1966). Patients with an internal locus of control believe that the outcomes of events are within their control (Rotter, 1966) and they would not attribute their high BG levels to external factors of stress and coincidence. For instance, a male patient illustrated the point of being a doctor for himself by giving examples of the food preferences and food choices that he made on a daily basis:

I mean this plate is small or large; I know myself. If I like to eat Konafa (a type of sweet), I will eat three spoons maximum because more than that is not good for the person without diabetes too. The fruits too. Some people eat the whole jar of figs or peaches without thinking. Here you are the doctor for yourself. The fruits are available now in the summer, and all the people eat figs and grapes that raise blood glucose. If you eat orange today don't eat an apple, so the person is the doctor for himself (Male patient 1. FG 1).

Male patient 1 who had been diagnosed with diabetes for more than ten years reported that he was able to take control over his dietary practices and maintain adherence to the recommended diet. He also emphasised his active role in controlling his diet despite the abundance and availability of foods with a high sugar content during summer that presented challenges for patients with diabetes to adhere to a diet plan. As is apparent in the quote, he sought to become an expert in his condition by understanding the dietary recommendations for patients with diabetes.

In this section and previous sections, I focused on the importance of developing programmes based on the views and expressed needs of the target audience, which are delivered in a format which is accessible to all and which is culturally sensitive, which builds on the knowledge and experiences of the group to seek to empower them to manage their diabetes. Patients in this study mentioned a number of other sources of health information besides HCWs which they had used to increase their awareness of diabetes. In the following category, I present and discuss sources of health information.

\subsubsection{Sources of health information}

More than half $(n=20)$ of the patients gained their information about diabetes from different sources rather than formal education sessions. For example, approximately a fifth $(n=6)$ of the patients in this study, including those aged between 40 and 70 indicated that the Internet was one of their main sources of information about diabetes. Male patients were more likely to use the Internet than females: 
From the Internet, I use Google to search the kinds of food that increase blood glucose and good food (Male patient 3. FG 7).

From YouTube. For example, what are the benefits of? Search by Google, and it gives me the information such as what are the benefits of walnut or almond.........Linseeds, onion, garlic and ginger. I keep reading and applying then I test my blood glucose, and I find it low (Female patient 1. FG 6).

These patients mainly relied on Google as a search engine to obtain their information about diabetes. The casual search for health information on the Internet was dominant among them. They found the Internet an easy and accessible source for all the information they needed to learn about diabetes. They also felt satisfied by contributing to their own knowledge and having an active role rather than relying only on the information received from HCWs. These findings may suggest that patients have developed their own expertise in the management of diabetes by seeking information to enhance their own health, which could be considered as a self-care initiative. These findings also suggested there is a shift away from the traditional sources of health information, such as HCWs, to impersonal sources of information such as the mass media.

The most frequently searched topics on the Internet by patients were: herbs that lower BG, the effect of food on BG levels and side-effects of medications. However, none of the patients mentioned that they received any recommendations about the Internet regarding credible sites for information from their HCWs, or that they accessed specific educational websites such as the National Diabetes Centre or the Ministry of Health in Jordan:

No, I never heard that the National Diabetes Centre has a website (Male patient 2. FG 2).

No one told me about these websites. I am hearing that for the first time. Are they useful? (Female patient 4. FG 5).

Most of the patients in this study were enthusiastic and motivated when I recommended examples of credible, educational and national health websites. For example, leaflets and pamphlets about diabetes are available on the National Diabetes Centre website to download. Diabetes and Health Magazine is also issued in Arabic every three months, and it is available on the centre's website, or patients can receive a hard copy when they visit the National Diabetes Centre, but few patients knew about this. 
Patients mainly relied on Google as a general search engine and YouTube to increase their awareness about diabetes, which increases the chances of accessing inaccurate information.

A few patients $(n=4)$ reported that the Internet might provide them with inaccurate information:

The information can be taken from everywhere, but sometimes the information from the Internet may be incorrect. For example, they say blueberry is good and cure diseases ...... but blueberry is a type of fruits that may increase blood glucose. Patients with diabetes should know the fruits that increase blood glucose (Male patient 3. FG5).

He should stay with his doctor and not try to use the Internet for example because it makes the person confused frankly (Male patient 1. FG 1).

These patients expressed their concerns about the accuracy and quality of health information that was obtained by the Internet. Patients found many recipes that aimed to reverse diabetes without an evidence base to support these recipes in the literature. Patients also received conflicting information from different websites that made them confused.

My observations showed that patients did not receive any information about credible websites from HCWs.

Doctors, nurses, pharmacists and dietitians did not refer any patients to online resources (Field notes).

Therefore, HCWs could usefully guide the patients to gain accurate and accessible information from recommended, credible online resources. However, a pharmacist interviewed never referred the patients to online resources:

When the person is old who can't open the Internet or open the computer, I do not think. It may work with young people maybe young people who are aware, educated so you can talk to them about this but when an old person comes to you, what you are going to tell about the online resource? (Pharmacist. Interview 6).

This pharmacist displayed opposing views regarding older people's access and use of the Internet as a source of information and therefore missed an opportunity to inform these patients of credible online sources. She was not interested in referring patients to online resources as she thought that old people were not able to use the Internet due to computer illiteracy. However, most of the patients in this study including those who were aged between 40 and 70 indicated that the Internet was one of their main sources of information about diabetes. 
Therefore, low education level and age were not barriers among patients to seek and access information on the Internet. Patients' family members may also be able to access the Internet for those who are unable to do so:

From TV, the Internet and health programmes (Female patient 5. 55 yrs. No education. FG $1)$.

In addition to the Internet, patients reported gaining health information from other sources:

Usually from patients with diabetes and the doctors (Male patient 4. FG 6).

My mother has diabetes, so we know what the forbidden foods are and what foods that we have to avoid (Female patient 6 . FG 1).

Patients indicated that social interactions (interpersonal relationships) were a good opportunity to exchange knowledge especially with other patients with diabetes. As I discussed earlier in this chapter, my focus group discussions were a good example of seeking information through social interactions with other patients. This female patient reported that her mother with diabetes was considered a good source of information. Her response showed that the experience of a family member with diabetes made her aware of the factors that increased BG levels. A cross-sectional study aimed to evaluate the awareness of diabetic retinopathy among a sample of 237 patients with Type-2 diabetes in Jordan. This study's findings reported that family members and friends with diabetes were the main sources of information about diabetes among the study sample (Bakkar et al., 2017).

A physician expressed his concerns about the information that patients received from other sources:

Patients may stop taking medications based on information from others such as 'this drug may harm your kidney or heart' (Physician. Interview 1).

Other people's experiences of side-effects of medications had a negative impact on patients' adherence to their medication regimen. A few patients stopped taking medications such as Metformin and insulin based on inaccurate information from family and friends as previously discussed in section 5. 5 .

A few patients $(n=3)$ believed that the doctor's knowledge was superior to other sources of information as in the following data extracts: 
Enough information I ONLY take it from the doctor, not from the Internet or other sources (Male patient 5. FG 4).

The information should be taken from the specialist who is the person who gives you the most benefits (Male patient 2. FG 5).

These patients perceived that the information received during medical consultations was more credible and trustworthy than the information gained from other sources. These patients stated that the doctors were solely responsible for giving health information to them. They were concerned that other sources might provide inaccurate and conflicting advice. Patients' perceptions of the doctor's knowledge were affected by the dominance of the medical model of health. Therefore, they sought their knowledge only from the doctors without acknowledging the input from other sources.

The amount of information that was conveyed to patients by doctors during consultations was varied due to work pressure, work demands and whether patients asked questions. Furthermore, there were essential requirements of patients that doctors had to complete in most consultations such as transferring the laboratory results to the patients' records, writing medications and request forms which affected the patient-doctor communication. Most of the patients who did not ask questions during consultations received limited information. The majority of conversations between the doctors and patients I observed focused on medications, diet and any immediate health concern, and for some patients, this was their only source of information unless they were referred to the dietician.

In my study, all patients reported that the information they received from the doctors and dietitian was easy and understandable:

The information is very easy (Female patient 2. FG 6).

The information is easy and valuable. I get brochures from the centre from the dietitian specifically. We read and study these brochures at home (Male patient 5. FG 8).

Patients mentioned that HCWs used simple and easy language during consultations. During conducting this study, I did not observe the dietitian or doctors or nurses giving patients brochures. However, many patients in the FGs mentioned that the dietitian provided the educational leaflets, and patients I interviewed reported reading them. My observations in the consultants' rooms supported patients' views that doctors used the simple information to communicate with patients. 
Patients also reported a number of sources of herbal information to increase their awareness of the health benefits of herbs. For instance, the Internet, TV, family, friends, and information that was passed down from the older generation. The Internet and the media were mentioned by 9 patients. None of the participants mentioned that HCWs were a source of information about herbs. A typical comment included:

From the Internet and from patients with diabetes who say that cinnamon is very useful. I drink one cup of it in the morning (Female patient 4. FG 5).

This study revealed the influence of family and friends (interpersonal relationships) on patients' decisions to seek complementary health care options. Recommendations regarding herbal usage were mainly sourced from the TV, family and friends. This implies that the use of herbs as a health seeking behaviour was shaped by patients' social environment. It is important to mention that the types of complementary medicine that participants were referring to were herbs, spices and plants. Most of the participants used the term 'herbs' or 'herbal medicine' to refer to herbs, spices and plants that were used by patients in this study. More than a quarter of all participants $(n=14)$ mentioned that patients who used herbs gained their information about herbs from family and friends rather than formal education sessions or HCWs. Similar findings were reported by 29 Omani patients that they only obtained their information about herbs from family (Subhi et al., 2016).

Besides health education to enable patient empowerment, participants suggested continuity of care to be introduced in the centre which they believed would improve patients' quality of care. The following category presents and discusses the continuity of care as reported by participants.

\subsubsection{Continuity of patient care}

Seeing the same doctor was suggested by approximately more than half of the patients $(n=21)$ as a facilitator to promote continuity of care and improve their experience in receiving care in the centre. None of the patients in this study was registered with a named doctor:

I see different doctors which is another challenge. Ifeel comfortable with one doctor rather than seeing a different doctor. I need time to explain my story to one doctor then the centre sends me to a different doctor who doesn't take time to read back my life history and I don't know the new doctor (Male patient 2. FG 2). 
The reception desk does not send you to the same doctor whom you feel comfortable with. I need the same doctor. I do not want another doctor to explain to him everything. I want the doctor who has known my case for two years (Female patient 5. FG 3).

Patients were evaluated by more than one doctor, but there was poor coordination between them. The most common problems with lack of continuity of care observed by me and reported by patients were: different doses of medications, conflicting advice, length of waiting time to be seen, lack of patients' satisfaction and incomplete medical records.

Conflicting advice and subsequent patient confusion were reported by patients and observed by me as a result of discontinuity between different doctors either in the centre or different health care facilities. The most common conflicting views that were identified by patients in this study related to medication doses and regimen:

The doctor told a patient with HbA1c of $8 \%$ to see the dietitian for health education. During the writing of the prescription, the doctor wrote down Amaryl 1mg x 1 tablet daily, but another doctor told the patient in the last month half tablet $0.5 \mathrm{mg} \times 2$ tablets per day, and it was not written in patient's records, so the patient was confused (Field notes).

Although the actual dose per day was the same, it was presented in a different way, and it may depend on what strength of the tablet the patient had been provided with at the last visit.

Sometimes, the original doctor wrote 60 pills but when I went to another doctor he wrote 30 pills, and I didn't know why he wrote 30. So I gave him the file to write all medications, but I get surprised every month with different medications (Male patient 2. FG 3).

If you see the same doctor the doctor knows all of your C.V, and she writes with red pen notes such as I have hypertension, and I have heart diseases. There are things that I told the first doctor. For example, I had numbness in my hands, and I asked her did you add the medications for nerves? She added it at the end of the file, and when I went to another doctor, she did not pay attention to it (Female patient 3. FG 6).

Lack of coordination of care between different doctors in the centre placed these patients at a high risk to receive different medications and doses. These patients' medication regimes were reviewed by different doctors who did not communicate with each other effectively which resulted in patients' confusion and being unclear about their medication regimen. There was also a discontinuity of information as a few doctors did not complete patients' medical records effectively following the medical consultation. These patients were aware of the poor 
coordination among HCWs and types of medications that they received. As is apparent in the quotes, quality of care, adherence to medications and patient safety were affected by fragmentation and lack of continuity of care.

Approximately a third of the patients $(n=14)$ in my study had more than one condition such as a stroke, hypertension and heart problems besides diabetes. Therefore, they were more likely to receive care from different health care facilities. Discontinuity in the management of their care was also experienced by a few patients $(n=3)$ who also visited the hospitals for their care:

A patient was taking Omeprazole for a year, and the doctor was surprised about that as the doctor in the hospital wrote Omeprazole, and it was not written in the patient's medical records (Field notes).

In addition to medication errors, there was the discontinuity of information and lack of communication between primary and secondary care. As a result of this discontinuity, this patient was confused, and his adherence to medications was affected by receiving contradictory advice from different doctors. My observations in the consultants' rooms supported patients' views that the quality of care was affected by this discontinuity in care management:

The doctor was unhappy because she requested weight counselling in 2015 and there was no follow-up from other doctors (field notes).

This patient had weight issues; therefore the doctor wrote in his medical records to be referred to the dietitian on the next visit. However, none of the other doctors followed up and monitored his weight problems or referred him to the dietitian over at least one year. Poor follow-up did not facilitate the transition of this patient's care needs to other doctors. Therefore, discontinuity of care hindered early recognition of health problems and resulted in increased gaps in care. A nurse reported how the centre policy hindered continuity of care:

There is a problem when the patient comes to the medical registry, and he does not have one doctor. Based on the patient's turn, he will see the doctor. If the patient wants to see the specific doctor, he has to wait for a long time. When the patient feels comfortable with one specific doctor, the next medical appointment he will see another doctor, and he may not feel happy. If the patient wants to register his name with one specific doctor, the registry will tell him that he has to wait for his turn. The patient will lose the hope of seeing the doctor, and he asks the registry to write his name on the list of any available doctor....... This process pushes patients to take their medications and leave (Nurse. Interview 4). 
None of the patients were registered with a named doctor; therefore, this policy negatively influenced the continuity of care. Patients were dissatisfied with this policy because of the length of waiting time to see a named doctor. From the nurse's account, this meant that some patients left without seeing any doctor and they had a poor engagement with their treatment plan. These patients' concerns about seeing different doctors every time were also reported by another study conducted among 20 patients with Type-2 diabetes in Kuwait. Approximately a third of patients experienced discontinuity of care which made them feel dissatisfied with the services provided (Jeragh- Alhaddad et al., 2015).

More than half of the patients $(n=21)$ suggested that continuity of care would increase their satisfaction, and enable the doctors to identify their individual needs and improve their outcome of care:

Because every time you see a new doctor, you need one hour to explain what is and what I was taking. He also spends one hour in reading your file and this yes and this no. However, when you see your doctor, he knows what you take what is this and what is that. I suffered from changing doctors because I spent one hour to explain this I take and this I do not take (Female patient 3. FG 3).

My experience with different doctors is negative. Because I feel psychologically comfortable with a particular doctor, so why I should go to see another one to tell him everything? I tell them to put my name to a particular doctor, but they reply: the number is full, and many patients are waiting for the doctor, and I want a particular doctor (Female patient 2. FG 6).

These patients reported that they disliked having to repeat their medical history to several doctors; therefore, they preferred to see a known doctor. Doctors had to spend more time to read through unfamiliar patients' medical records which were too time-consuming for the patients and also for the doctor. Female patient 2 was dissatisfied with switching doctors from time to time. Lack of continuity affected interpersonal communication as patients did not feel comfortable seeing different doctors. These patients also felt that this centre's policy impeded their efforts to see the same doctor that may yield medical, emotional and perhaps physiological benefits for them.

The centre policy was considered by patients as an organisational barrier because it did not encourage them to seek continuity of care and establish a continuous relationship with their preferred doctor. Also, this policy did not consider the psychological aspect of the patient's health as many patients expressed their dissatisfaction with the lack of continuity of care. In 
this study, most of the patients preferred to see the same doctor as they reported feeling comfortable and safe being treated by a familiar doctor who also knew them. Patient-centred care means that treatment and care should consider patients' preferences and needs (Richards, Coulter, \& Wicks, 2015). However, the centre policy did not promote patient-centred care as patients' needs and preferences were not met. Therefore, the main aspect of care provided was writing medication prescriptions as the doctors had limited time to review medical records of unfamiliar patients:

I prefer the doctor who follows my case. I only visit one place but the problem the doctor may be present or absent. Absenteeism of my doctor affects us because he follows your case and knows what you want. Sometimes you come to the new doctor carrying the treatment booklet, so this doctor only writes the medications on the prescription paper (Male patient 6. FG 4).

This patient felt disappointed as he was not able to discuss other health issues related to diabetes with the doctor. Many patients used the term 'my doctor' which implied that patients sought continuity of a therapeutic relationship with one doctor. Patients' responses showed how this centre policy did not support continuity of care, and as a result supported the medical model of care as opposed to a social model of care by focusing more on prescribing medications than building a long-term, patient-doctor rapport.

The supervisor of Health Promotion suggested that continuity of care is important for delivering comprehensive health care which then, in turn, will support applying the wholeperson approach in primary health care:

I am with having one family physician who knows history and comprehensively treats patients. Psychologically is better when the doctor knows all the medical history and in case of happening a new thing, he knows how to treat it. So I am with non-duality and against pluralism in resources. Enough. Even the patient himself feels comfortable with a certain doctor, and he asks every time to see him. So this is a positive thing one doctor, not pluralism (Supervisor of Health Promotion. Interview 10).

This supervisor mentioned that registering patients with a named identified doctor would familiarise the doctors more with their patients' history. This will assist the doctor in the early identification of any changes in the patient's health status. In addition to the medical benefits of continuity of care, patients will feel psychological comfort and increased satisfaction which is consistent with the whole-person approach where the physical, cultural, spiritual and social 
aspects of health are interconnected and integrated (Bertalanffy V, 1968). In adapting a wholeperson approach, the patient will be seen as a whole system of interconnecting aspects rather than a set of disjointed parts (Mele et al., 2010). Therefore, continuity of care could be utilised to support the essential components of a patient's health; physical, psychological and social aspects as proposed by the WHO (WHO, 1948).

Informed by patients' responses and concerns regarding the lack of continuity of care, I elicited the views of the receptionist and the director of the centre:

A patient visits clinic 1, and he feels comfortable, so he wants to come back to clinic 1. One day the doctor in clinic 1 is absent, so I send this patient to clinic number 3. When he enters this clinic for the first time, he feels uncomfortable. In the next visit, if the doctor in the clinic 1 is absent, this patient will say I do not want to receive treatment, and I want to go home, and I come back when the doctor in the clinic 1 is here. When the patient visits the doctor in clinic 1, and the doctor shows care to him the patient will keep wanting to go the clinic number 1. When I tell him I cannot send you to clinic number 1, I will send you to clinic number 3 because the clinic 1 saw 30 patients and clinic 3 saw 20 patients, so there is ten patients difference. The result is one doctor does work, and another is resting. So I make sure all the clinics work the same for balance (Receptionist. Interview 9).

The reception staff made sure that all family medicine clinics saw the same number of patients every day and all the doctors shared the workload equally. To achieve balance in workload and numbers of patients between clinics, patients had to see different doctors every month based on their appointment. This receptionist perceived that continuity of care is difficult to accomplish due to workload. This centre's policy focused more on the needs of organising the work than the needs of patients in seeing the same doctor. Each doctor had to provide care for 30 to 50 patients with chronic conditions in an average clinic day. Therefore, workload and discontinuity of care were also exacerbated by lack of an appointment system to maintain a balanced number of patients between different clinics. Providing a specific appointment time for patients to attend the centre was suggested by a patient's spouse:

Surely, surely. Any patient with diabetes or anyone should have a specific appointment. The patient comes at a specific time. Your time from ... to ...... So the patient feels he is human, Ifeel I am a valuable person (Spouse. Interview 3).

During the informal discussion with the director of the centre, he replied to my questions about patients' needs to be registered with a named doctor: 
A change in the centre policy is planned to aid continuity of care (Field notes).

However, approximately one-fifth of the patients $(n=7)$ indicated that seeing several different doctors did not affect their treatment plan and all doctors had similar capabilities. They indicated that seeing different doctors can also be beneficial as they may offer another opinion:

I do not see it as a problem because my records are the same and the routine of care is the same as the doctor writes down the same treatment and all the same (Male patient 5. FG 4).

However, I say from a different perspective that every doctor has his own style and method. For instance, in the last visit, the doctor warned me that my blood glucose was high and he wanted to know the reason why the blood glucose was high then he reached to the result that I was eating one meal a day. I was visiting many doctors before, and no one told me about it (Female patient 1. FG 7).

Male patient 5 perceived that all information was written in his medical records, therefore seeing different doctors would not affect the quality of care. In his perception, seeing different doctors was not essential, and continuity of care can be achieved by following-up notes in the medical records. However, incomplete medical records was one of the findings that was attributable to discontinuity of care and my observations also found that many medical records were incomplete and inaccurate (section 7.3). Female patient 1 reported that her new doctor was able to detect the reason behind her high BG levels while the previous doctors did not consider her dietary requirements. Therefore, from her perspective, a variety of doctors improved her quality of care and outcomes.

Most of the patients in the FGs reported that seeing different doctors negatively affected their treatment plan. In order to avoid that, they addressed the need for continuity of care to be recognised and facilitated by the centre policies. Seeing a familiar and trusted doctor is the most effective way of providing consistent information and continuity in treatment in primary care (Cowie, Morgan, White, \& Gulliford, 2009). A systematic review conducted by Alsairafi et al. (2016) which focused on the management of Type-2 diabetes in the Middle East reported that continuity of care is also an important element to enhance rapport and confidence in the patient-HCWs relationship. My study findings revealed several types of discontinuity of care (information, interpersonal and management) that are also mentioned in the literature (Cowie et al., 2009; Freeman \& Hughes, 2010). 
Discontinuity of information is defined as the unavailability of accurate information from one health care facility to another (Freeman \& Hughes, 2010). Incomplete patients' medical records were an example of lack of informational continuity in my study. Interpersonal discontinuity refers to a lack of an ongoing doctor-patient relationship due to not registering patients with named individual professionals (Freeman \& Hughes, 2010). For instance, all patients in my study were not registered with a named doctor which made interpersonal continuity difficult to achieve. Management continuity is defined as the consistency of clinical management, including providing and sharing information and care planning, and any necessary coordination of care required by the patient (Freeman \& Hughes, 2010). It includes coordinating, communicating, integrating and personalising care between different doctors and organisations in order to deliver high-quality care for patients (Cowie et al., 2009). In my study, patients received conflicting advice especially related to medication doses and regimen from different doctors in different health care facilities.

In addition, patients, their carers and HCWs suggested facilitators at the health care system level to improve management of diabetes which are discussed now.

\subsection{Facilitators at the health care system level}

Within this subtheme, there are three main categories: improve management of diabetes at the health care system level, the role of the mass media and settings for health education.

Firstly, I present and discuss the suggested strategies to improve diabetes management at the health care system level.

\subsubsection{Improve management of diabetes at the health care system level}

A male patient suggested the need to establish a separate clinic managed by diabetes specialist HCWs to provide care for patients with diabetes:

Ministry of Health should provide a separate clinic which has only an expert health care worker in treating people with diabetes. So patients spend enough time with health care professionals (Male patient 1. FG 2).

This patient perceived that providing appropriately skilled staff in diabetes management and separate clinics from general family medicine would improve the management of patients with diabetes. There was a nurse in the centre who had completed a diploma in diabetes, but her skills and knowledge were not utilised as a diabetes specialist nurse (DSN) as she continued 
working as a general nurse. In the United Kingdom, the literature showed that DSNs play important roles in preventing diabetes complications, supporting people with complex health and social care needs and reducing hospitalisations (Diabetes UK, 2014). So it is a missed opportunity for the health care system in Jordan by not introducing and establishing diabetes specialist programmes for nurses. The provision of DSNs could expand the roles of the nurses at the centre to provide health education, which in turn would reduce the workload on doctors, improve continuity of care and provide the patient with an opportunity to discuss other health needs with nurses. A dietitian reported that the primary health care centre should reach patients who were not able to attend the centre for different reasons:

Community and the centre should follow patients' progress. We as HCWs should reach the patients if they cannot reach us (Dietitian. Interview 3 ).

This dietitian referred to the role of community nurses who visit and provide care for patients in their own homes. Home visit programmes by community health nurses are available in Jordan mainly to assist in early childhood and child development (Kawafha, 2016). There are also community health nursing modules as part of the undergraduate nursing programme to promote the health of the local community (The University of Jordan, 2018). During my observations, the doctors wrote medication prescriptions for many patients who did not attend the centre, but whose relatives had attended to gain the medication prescription. Most of the patients were also older who were not unable to attend the centre alone as they needed a family carer to accompany them every time they came. Additionally, further diabetes complications may develop as a result of the doctor not seeing the patient for a long time:

A patient's spouse came to take her husband's medications, and he has not come to the centre since 2015, but the doctor refused to write the medications because she wanted to see the patient (Field notes).

Multiple trips to the centre for obtaining all written medications in the prescription was mentioned by many patients and observed by me as a challenge related to the management of diabetes. A male patient suggested that all medications should be available in the centre at the time of prescription refill (section 7.2.2.1).

A male patient and a nurse suggested a need to establish a society for patients with diabetes:

There should be a support group for patients. I wish if there is a support group that provides care for patients in this region because we feel lonely (Male patient 2. FG 3). 
I prefer if there is a support group for patients with diabetes. For example, patients who take insulin should create groups with their friends and relatives, and they show cooperation between them. That will improve the patient's spirits, and the patient knows there are people around him know about his condition (Nurse. Interview 7).

These participants reported that the establishment of a patients' support group would help in building-up relationships between different patients and with their family carers. They believed that a patients' support group would also provide social support to members and raise awareness of diabetes amongst the wider community and population in Jordan. However, there is a voluntary patients' support group in Jordan called the Jordanian Society for the Care of Diabetes (JSCD). Membership of the JSCD is open to all members of the community and patients. There is also a Facebook group for all members of this society to encourage patients to share their needs and concerns with others. Examples of activities that were conducted by the JSCD in 2017 were free essential health checks days including feet and eye examination, and public lectures and workshops (Jordanian Society for the Care of Diabetes, 2018). None of the patients, their family carers and HCWs reported awareness of this society or its existence. Therefore, there is a need to promote this society more widely.

Patient support groups may offer a variety of services, including online resources, consultations, group therapy, team building activities, and other resources to teach individuals how to cope and adapt to the lifestyle changes due to diabetes (WHO, 2018d). Diabetes UK and the American Diabetes Association are examples of patient support groups.

In this section, patients and HCWs suggested facilitators that went beyond a focus at an individual level by recognising social and environmental interventions. They reported that health promotion interventions at the health care system and community levels would facilitate a health-enabling social environment which may lead to encourage patients to take control over their own health. The socio-ecological model (McLeroy et al., 1988) discusses the health promotion interventions that aim to establish a health-enabling social environment. Therefore, it is important to consider health promotion interventions at the community level rather than just the individual level to improve patients' health.

In addition to improving management of diabetes at the health care system level, patients' and HCWs' responses showed that health promotion strategies could be achieved by addressing diabetes at a population level. The mass media was believed by patients and HCWs as an 
effective tool to raise public awareness about diabetes. The role of mass media is discussed now.

\subsubsection{Role of the mass media}

The mass media includes any audio-visual or printed material that targets a wider audience such as television, radio, posters and brochures (Naidoo \& Wills, 2009). TV, posters and brochures were reported by a few patients and HCWs as tools to raise awareness of diabetes among the general public. A nurse perceived diabetes as a national burden; therefore, health awareness should be delivered at a population level by using mass media tools such as TV:

I like using the TV for health education. Before there was a TV series about diabetes and people liked it...... TV series contain information about diabetes, types and complications. I think patients gained much information because it was easy and was seen while people at home. I think it is the easiest way to behavioural change and delivering information about diabetes for patients who are not able to come to the centre (Nurse. Interview 4).

This nurse perceived that using TV to deliver health-promoting messages would target a wider audience by enabling them to watch programmes about diabetes at home. Therefore, these programmes may eliminate the barriers that hindered some patients' ability to attend health education sessions in the centre such as time, cost, transportation and distance. However, the health centre was not providing any educational programme at the time of conducting this study. She mentioned that patients showed satisfaction with the previous TV cartoon series about diabetes (section 8.2.3). She also reported that television could be used to deliver quick and simple health message to achieve behavioural change. However, the literature shows that mass media tools have more impact on short-term behavioural change regarding physical activity and diet, but they are less effective to achieve sustained behavioural change (Marcus, Owen, Forsyth, Cavill, \& Fridinger, 1998; Bauman, Bellew, Owen, \& Vita, 2001; Naidoo \& Wills, 2009; DiClemente et al., 2009). Some factors may hinder using the mass media to achieve sustained behavioural change such as the influence of social norms and habits (Wakefield, Loken, \& Hornik, 2010). ). In chapter six, the power of social and cultural customs in relation to food shaped patients' food preferences and also contributed to a high blood glucose level among the patients in my study. Therefore, mass media may not achieve longterm behavioural change without partnership and support from the surrounding community, policy changes and environmental strategies (Naidoo \& Wills, 2009). A male patient also 
discouraged utilising TV in delivering health promotion about diabetes as patients may not watch TV:

Not everyone watches TV such as if I am not interested in watching this health programme I will change the channel (Male patient 4. FG 6).

Male patient 1 reported one disadvantage of mass media as patients may turn off their TVs. Therefore, it could be difficult to seek immediate feedback from the patients, and the message could also be misunderstood and dismissed as the mass media is one-way communication (Naidoo \&Wills, 2009).

There was a difference in opinion between patients' and HCWs' views regarding written educational materials such as brochures and posters. A nurse and the supervisor of Health Promotion valued printed material, but that it is needed to be explained to the patients first, not just handed out without an explanation:

We explain to him what the content of the brochure is ...ok...then I give it to him. However, when you give it to him without explaining what inside the brochure is, he will not gain any benefits since he reads it as superficial information (Supervisor of Health Promotion. Interview 10).

She perceived that the brochures would complement and support the one to one consultation with patients. Approximately half of the patients $(n=16)$ reported that they supported receiving brochures and having posters displayed in the centre.

Posters and brochures are useful. Clear posters and about diabetes such as, how to administer insulin and when you have to take medications. For people who are illiterate, the poster contains sun or night, so they can know the right times of taking medication (Male patient 2. FG 2).

I gain benefits from reading brochures (Female patient 4. FG 7).

Male patient 2 reported that a poster should be written in easy and simple language that patients can understand. On the other hand, one physician and one nurse did not support the idea of distributing brochures to patients because of their view that patients lacked self-motivation to read them:

We do not have posters, and nobody reads in this centre. If you give the patient brochures, he will not read, and it will be discarded in the dustbin later (Physician. Interview 1). 
Posters and brochures were not provided in the centre at the time of data collection. This physician perceived that patients were not self-motivated to read brochures which would be wasted. During my observations, there were no posters or any educational material about diabetes or chronic conditions in the waiting area. There were posters about hand washing technique and the recommended body and arm positions of the patient for measuring blood pressure in the nursing room in the centre. This variation in the responses of patients and HCWs may indicate a gap in communication as a group of HCWs did not consider patients' educational needs, and they held preconceived views regarding patients' preferences. Therefore, HCWs missed an easy, relatively inexpensive opportunity for health awareness as patients on average waited for three hours before they entered the consultant's room for their appointment. The presence of posters or information shown in the waiting area may also assist HCWs in educating patients and their family members who attended the appointments with their relatives. A systematic review of 15 studies that focused on the effectiveness of posters on changes in individual's knowledge, attitude or behaviour reported that posters produce the highest effectiveness in knowledge transfer when integrated with other educational methods (Ilic \& Rowe, 2013).

In addition to the role of mass media to promote the public's health, a few patients, patients' family carers and HCWs suggested that health education could be delivered in a wider context to target the population in different age groups. The following category presents and discusses the suggested settings for health education as reported by participants.

\subsubsection{Settings for health education}

Approximately half of the patients $(n=18)$ reported that the comprehensive health care centre is the most suitable place to conduct health education sessions:

I prefer the centre because it is closer to us (Female patient 4. FG 3).

All families in this region have medical records in the centre, and people with diabetes are known cases. The best place is the centre (Male patient 5. FG 4).

Availability of HCWs, accessibility and known cases of patients were reported by patients as the main reasons why they preferred the centre as a place to conduct health education. A few patients $(n=4)$ and a patient's son suggested that health education should be delivered in a wider context to target the population in different age groups. Other sectors such as schools, universities, mosques, and the Dewan (a place where people meet and gather) were perceived 
as public places that could seek to promote health among a large number of the population in the Jordanian society:

They should talk about diabetes in the Friday speech in the mosque and use the religion in promoting health (Male patient 2. FG 2).

A few people stopped smoking after showing pictures about the effect of smoking on the lungs in the mosque. I wish if they show pictures about diabetes complications in the mosque (Son. Interview 1).

This patient's son and male patient 2 were aware of the positive role of religion in promoting health. Attending Friday Sermon is a religious obligation for men, but women can also pray in the mosque. Therefore, it could be utilised to increase the public's awareness of diabetes. One of the objectives of the National Strategy and Plan of Action against Diabetes in Jordan 20112012 was involving community leaders and religious leaders to support the implementation of this strategic plan, so this suggestion is congruent with the national strategy (MOH, 2012).

The literature showed that the use of the church for health promotion is effective for modifying unhealthy behaviours, increasing knowledge and early screening for chronic conditions (Kelly \& Huddy, 1999; DeHaven, Hunter, Wilder, Walton, \& Berry, 2004; Harmon, Kim, Blake, \& Hebert, 2014). A qualitative study explored the educational material disseminated by 11 African American churches (Harmon et al., 2014). This study's findings showed that churchpublished material was the most trusted and frequent source of screening information among African American communities. Screening activities were also promoted within these churches.

Many of the Qur'an's phrases and Islamic beliefs also encourage patients to seek treatment and take responsibility for their own health through maintaining a balance between physical, psychological, social and spiritual aspects which is consistent with the essential components of health as proposed by the World Health Organisation (WHO) in 1948 (WHO, 1948; Nabolsi \& Carson, 2011; Albargawi, Snethen, Gannass, \& Kelber, 2016). A qualitative study explored perceptions of 12 community leaders regarding the roles of Imams (prayer leaders/Sermon giver) in promoting health among American Muslims (Padela, Killawi, Heisler, Demonner, \& Fetters, 2010). This study's results showed that most of the participants viewed that Imams can play an important role in health promotion by encouraging healthy behaviours through scripture-based messages on Friday Sermons and through Islamic events. The idea of utilising religion in promoting health and delivering health-related messages is not new in Jordan. In 
1996, the WHO published the 'Amman Declaration on Health promotion' that discussed health promotion through Islamic lifestyles (WHO, 1996). Each statement of this declaration is supported by evidence from the Qur'an and Hadith of the Prophet Muhammad. For example,

Statement (6): "Healthy nutrition firstly consists of a balanced diet in terms of quantity. Excess in food is harmful to health because it leads to diseases of affluence, which is against Islamic guidance.” (WHO, 1996, p. 17):

As cited in the Qur'an:

Eat and drink but avoid excess (Qur'an 7:31).

In addition to utilising mosques to encourage healthy behaviours, schools were suggested to be used in promoting health especially among young people:

To save money, health education should start in the schools, and it should be part of school curriculum. We should have educational programmes for school kids that they will be able to educate their parents about diabetes (Physician. Interview 1).

This doctor emphasised that chronic conditions awareness should be part of the Jordanian school curriculum that could reduce the financial burden of the treatment of diabetes. Introducing national health problems into schools may create more awareness in future generations that will enhance the role of family carers in patient's management of diabetes and hopefully reduce the prevalence of Type-2 diabetes as the next generation adopt healthier lifestyles. Engaging the younger generations is an effective tool to foster health and wellbeing among them (Wallace \& Kenkre, 2015).

Participants suggested that the utilisation of community leaders and institutions create a supportive environment for patients. Participants' responses were consistent with the multilevel interventions in the socio-ecological model that integrates health promotion strategies across all levels of influence and aims to modify the social norms and values (McLeroy et al., 1988). The socio-ecological model suggests integrating actions to support individual behaviour change incorporated within the community and environmental support. The socio-ecological model aims to maximise health promotion via all levels of influence to support individual behaviour change. For example, in Jordan, the Healthy Schools Programme has been established since 2008 aiming to create a healthy school environment (Royal Health Awareness Society, 2017). Health education, psychological and social support are examples of the areas that are covered in this programme. This programme is an example of introducing the 
health promotion concept into the school environment by targeting younger people in society. This school programme is also compatible with the WHO healthy settings approaches for disease prevention and health promotion (WHO, 2018c).

The socio-ecological health model (McLeroy et al., 1988) was useful for aiding understanding of the facilitators which were suggested by participants at the community, organisational and policy level to improve patients' adherence to the management plan. The findings in this chapter indicated that patients, their carers and HCWs were aware of the influences at different levels; the interpersonal, community, social, organisational and policy level. They suggested interventions and activities operated at multiple levels to target community, policy and organisational change in the national health care system, the local health care centre, mosques, schools and community agencies. The findings also suggested that creating a health-enabling social environment will be achieved through broad systemic changes in national health policy and the health care system and its services. The socio-ecological model (McLeroy et al., 1988) discusses the health promotion interventions that aim to establish a health-enabling social environment. Therefore, it is important to consider health promotion interventions at the community level, organisation and health care system rather than just the individual level to improve patients' health.

\subsection{Summary}

This chapter has discussed the main facilitators of diabetes management from the perspectives of the patients, their family carers and HCWs. Participants suggested a wide range of facilitators at different levels of care. Facilitators were grouped into two main levels: at the local comprehensive health care centre level and the national health care system level. Participants perceived that addressing diabetes at a population level such as through schools, the mass media and religious places would promote public health and the health of the population in Jordan.

Most of the patients, HCWs and patients' family carers suggested that health education and patient empowerment (doctor for herself/himself) and continuity of care as strategies to improve diabetes management at the comprehensive health care centre. Patients and HCWs went beyond the centre level by suggesting facilitators and policies that target the population level. Improving the management of diabetes at the health care system, the role of the mass 
media and settings for health promotion were suggested facilitators at the health care system level.

The educational health programme and material which had been provided in the centre until 2015 were analysed and reviewed in this chapter. Drawing on the expressed needs and concerns of patients and family carers the content and format for a future educational package was proposed. Policies which promoted continuity of care was suggested by many patients to improve diabetes management and establish a continuous relationship with their preferred doctor within the comprehensive health care centre.

A few HCWs perceived diabetes as a national burden; therefore, diabetes awareness should be delivered at a population level by using mass media tools, mainly TV. Introducing health awareness to the school curriculum was perceived by a few HCWs and patients as an effective tool to prevent diabetes among the younger generation. Using religion and religious organisations to promote public health was suggested by patients. Using phrases from the Qur'an to support health promotion messages and also using the Mosque as a setting for health promotion. The Friday Sermon could also be utilised to deliver health messages to people.

The final chapter will report the main findings and recommendations as well as the limitations of the study. 


\section{CHAPTER NINE}

\section{CONCLUSION}

\subsection{Introduction}

This chapter includes a summary of the main findings, and the limitations of this study are discussed. This is followed by a discussion of the relevance and recommendations of the study findings. First I remind the reader of the aim and objectives of the study.

Aim

This study aimed to explore the reasons why glycaemic control was poor among a sample of patients with Type- 2 diabetes who used primary care services provided by a selected health care comprehensive centre in a large city in Jordan.

\section{Objectives}

The following objectives were set:

1-To explore the knowledge, attitudes and behaviours of patients with poorly controlled Type2 diabetes and factors affecting adherence to their treatment plans.

2-To identify barriers to Type-2 diabetes care among patients with poor glycaemic control.

3- To explore patients', family carers' and health care workers' (HCWs) perceptions of the factors that contribute to poor glycaemic levels among patients with Type-2 diabetes.

4-To identify patients', their family carers' and HCWs' views on factors which might facilitate improved glycaemic control levels among people with Type-2 diabetes in Jordan.

5-To inform the development of an educational package for patients with Type-2 diabetes based on information derived from HCWs, patients with poorly controlled Type-2 diabetes and their family carers to ensure patients' needs and concerns are addressed.

An ethnographic design was undertaken to understand shared and common experiences among participants in their social, cultural and economic contexts. Thematic analysis was employed to identify the factors associated with high BG levels among patients. The aim and objectives of this study were met using multiple methods of data collection with a range of different stakeholders which aided an in-depth understanding of the factors affecting the management of Type-2 diabetes in Jordan. First, a summary of the main findings is presented. 


\subsection{Summary of main findings}

Patients reported different approaches regarding how they came to be diagnosed with Type-2 diabetes. Most of the patients visited the health facility after experiencing signs and symptoms of diabetes. A few patients were proactive and visited the health centre to check their blood glucose levels, and the remainder were identified through opportunistic screening when attending a hospital for an unrelated condition. Stress was perceived by the patients as a cause of developing diabetes and as a reason for poorly controlled diabetes. Fatalistic beliefs and attitudes were expressed by patients when sharing their views about the causes of diabetes, the reasons for developing diabetes and their inability to control their blood glucose levels. Patients felt that diabetes is an inevitable condition due to stress and having a positive family history of Type-2 diabetes. Therefore, fate was used either as a stress relief or an acceptance or explanation of their inability to follow their treatment plan. Patients also associated fatalistic attitudes with religious beliefs and the power of God as illness and its progress was perceived as being in "God's hands".

Patients were aware of most of the signs and symptoms of diabetes as well as its main complications. However, there was a number of misconceptions expressed by approximately a third of patients $(n=12)$ regarding medications and the seriousness of the condition. Patients' misconceptions included that Type- 2 diabetes is a mild form of the condition and this perception negatively affected their level of adherence to their management. Patients also managed their condition based on noticeable physical signs and symptoms so when they did not experience symptoms of hyperglycaemia, they stopped taking medications, or reduced the dose of their medication. HCWs were aware of patients' beliefs and misconceptions about diabetes. HCWs reported that insulin was perceived by patients as a last resort and a sign of end stage of diabetes, so patients were reluctant to commence insulin therapy despite clinical need.

Patients reported the influence of family, friends and culture on their food preferences and the food choices that they made on a daily basis and during social gatherings. Engaging in social relationships usually included increased consumption of sugar-sweetened beverages such as fruit juice, and foods that contain high levels of fats and carbohydrates. Therefore, these dietary choices affected the ability of most patients to maintain adherence to the recommended diet as refusing food especially at social gatherings was seen as being culturally and socially unacceptable. Home eating habits, such as communal eating and the nutritional knowledge of 
family carers also influenced the foods that patients consumed. Approximately one-third of the patients $(n=11)$ talked about the influence of meal contents and the quality of food that were eaten either at home or provided to them by hosts, on adherence to their diet plan.

Social factors influenced engaging in physical activity and taking medications among patients. Walking was the most commonly reported physical activity. Female patients experienced more barriers in gaining access to gyms and health clubs than males. Exercising outdoors posed challenges for both male and female patients due to social embarrassment with physical activity and socio-cultural barriers which restricted women in particular from engaging in outdoor physical activities.

Most of the participants talked about health care seeking behaviours and patients reported their experiences of using complementary therapies, in particular herbs and plants. Although HCWs did not support the use of herbs or plants, approximately half of the patients $(n=18)$ reported using herbs and plants and often did not inform their doctor that they were doing so. Participants talked about the types of herbs and plants and the reasons for the utilisation of them by patients for the management of diabetes. The most common reasons for using herbs were feelings of comfort, as a perceived cure and for lowering blood glucose levels. Okra (Bamia in Arabic) was reported as the most common plant used by a fifth of patients who used plants.

Several challenges related to the health care system and its policies were mentioned, and the impact of those policies on service provision and patient care were also observed. These challenges such as medication insecurity and health insurance policies resulted in poor adherence to the management plan, multiple visits to the centre and patient inconvenience. Impact of medical insurance; having multiple insurance and lack of comprehensive care of the medical exemption policies. Medication insecurity; medication shortages and changing medications brands which resulted in patient fears, leading to hoarding of drugs. The last challenge was the unavailability of the main laboratory tests in the centre such as HbA1c test, postprandial blood glucose test, lipids profile tests and liver enzymes. These were reported as challenges at the national health care system level.

Participants also reported a number of wide-ranging challenges that hindered management of diabetes at health care workers (HCWs) level in the local comprehensive health care centre. The impact of different aspects of staff shortages and poor patient-HCWs communication on patients' management plan was reported and observed. Ineffective patient-HCW 
communication, work pressures, a long waiting list of patients and staff shortages were reported challenges relating to $\mathrm{HCWs}$ in the comprehensive health care centre.

Patients, their carers and HCWs suggested topics that they thought needed to be covered in any future health education sessions. Learning about diabetes-related complications was the most common topic reported by many patients. A few patients $(n=3)$ and a physician also suggested stressful factors, the emotional component of food and herbal remedies to be covered within any future educational programme. Approximately half of the patients $(n=16)$ preferred receiving health education by attending a focus group or group discussion over other sorts of formats. Approximately a quarter of the patients $(n=9)$ also believed that health education to be an effective tool in empowering them to take control over their own condition. The term 'doctor for himself or herself' was used by five patients, a receptionist and the supervisor of health promotion to describe their views on the benefits and values of health education which, in turn, they believed would enable patients to become experts on their condition.

Patients, their carers and HCWs suggested a wide range of facilitators for achieving good glycaemic control at different levels of care. Facilitators were grouped into two main levels: the local comprehensive health care centre level and the national health care system level. Participants perceived diabetes as a national burden; therefore, diabetes awareness should be delivered at a population level. Introducing health awareness to the school curriculum was perceived by participants as a useful tool for health promotion and to prevent diabetes among the younger generation. Using the mass media, mainly TV and religious places were also proposed to promote public health and the health of the population in Jordan.

Patients also recommended utilising religion and religious organisations to promote public health among the public. Suggestions included using phrases from the Qur'an to support health promotion messages and also using the Mosque as a setting for health promotion. The Friday Sermon could also be utilised to deliver health messages to people.

Policies which promoted continuity of care were suggested by many patients to be a facilitator for improving diabetes management and enable them to establish a continuous relationship with their preferred doctor within the comprehensive health care centre.

The socio-ecological health model (McLeroy et al., 1988) was useful for aiding understanding of the factors which influenced patients' experiences of Type-2 diabetes but also is useful for looking at the levels of health promotion intervention needed to be effective in facilitating behaviour change. Patients' misconceptions about diabetes, beliefs about medications, lack of 
awareness and negative attitudes towards diabetes were among intrapersonal factors that hindered adherence to the management plan. Patients' relationships and interactions with immediate family and friends especially regarding family support, sources of information and home food environment were among interpersonal relationships (McLeroy et al., 1988). The traditional eating customs, food culture, herbal usage and lack of culturally sensitive health clubs were among the socio-cultural factors that influenced patients' diabetes management behaviours at the larger social system. The national insurance system, medication supply problems, ineffective patient-HCWs relationships, staff work pressures and a long waiting list of patients were among the challenges related to the management of diabetes for these patients at the national health care system level.

Study participants emphasised the key roles of health promotion, the national health policy and settings for health promotion to improve the management of diabetes in Jordan at different levels (McLeroy et al., 1988). They suggested interventions and activities operated at multiple levels to target community, policy and organisational change in the national health care system, the local health care centre, mosques, schools and community agencies. The findings also suggested that creating a health-enabling social environment will be achieved through broad systemic changes in national health policy and the health care system and its services.

As with any research study, there were a number of limitations in this study. These limitations are explained now.

\subsection{Limitations of this study}

This study was conducted in one large sized comprehensive health care centre in a large city in Jordan. The target sample was patients who accessed the primary care services provided by the selected centre. This sample may not be representative of all patients with poorly controlled Type-2 diabetes in Jordan. Patients who also attended the centre are more likely to be concerned about their condition or may face different barriers than those who do not come to the centre. However, I was not attempting to produce generalizable data instead to provide an in-depth account of a particular sample in a primary care setting which may inform similar groups in similar settings. The findings from this study have direct relevance for the centre under study and certainly may have relevance for similar patients. Therefore, the study findings and inferences may be generalised theoretically (Mason, 2002) to other patients with poorly controlled Type- 2 diabetes in similar contexts. I have provided sufficient information about the 
context of the study setting which hopefully will enable the reader to judge the transferability of my findings.

The selection of one setting was considered appropriate for this study. However, the selection of one setting raised practical concerns related to confidentiality of participants' identities and study location. I addressed this by ensuring that the data were presented in such a format that the reader will not be able to recognise the participants' identities. Any quotes from the participants were assigned identifiers, and the study setting was also anonymised.

A limitation of locating the study in one setting was that the health education programmes had stopped in the centre for more than six months before I conducted my data collection. Attending these programmes may have yielded more comprehensive and rich data about the health promotion programme provided in the selected centre. However, I was able to gather patients' experiences of the health education programme retrospectively through focus groups. A dietitian who was an educator in the previous programmes was also interviewed to explore and gain an understanding of these programmes. Additionally, I also participated in the diabetes educational sessions provided in another centre, which I was informed was similar to the one which had been delivered in the centre under study. Attending these sessions enabled me to take notes about the information given and observe the style and format in which the information was delivered.

Recruitment of physicians was challenging in this study. There were five family medicine clinics providing care for patients with chronic diseases. Despite five participant information sheets being distributed to physicians, only one physician agreed to be interviewed. I had aimed to recruit more physicians $(n=3)$ who primarily delivered health education and provided care to patients with Type- 2 diabetes. The other physicians reported that they were not able to be interviewed due to workload and time pressures. However, most of the observations were conducted in the physicians' room. I also conducted informal conversations with the physicians during observations and in coffee breaks which with their permission further had informed the analysis of data and subsequent findings.

I consider the study sample of 38 patients, $10 \mathrm{HCWs}$ and six family carers was adequate and appropriate for addressing my research aim and objectives. My sampling strategy was purposive, and I applied the criteria of appropriateness and adequacy in selecting my sample (Morse \& Field, 1996). I invited and included the most knowledgeable participants regarding this research topic to participate, and the sample size was considered adequate for addressing 
the research aim and objectives. The relevance and recommendations of the study findings are discussed now.

\subsection{Relevance of the study findings}

Hammersley (1992) proposes relevance to human interest as an essential criterion to evaluate ethnographic research products. According to Hammersley (1992), there are two features of relevance: the importance of the topic which has to be within the substantive field and the contribution of the research findings it makes to the existing literature. Ethnographic research findings have to address the expectations of different potential audiences such as patients, the research community, policy makers and practitioners. Hammersley (1992) argues that fellow researchers are the immediate and most important audience for research findings. In my opinion, patients with Type-2 diabetes, particularly those with poor glycaemic control and practitioners, are given privilege over relevance for other audiences as my purpose in conducting this study is to add to the body of knowledge to inform the future care provided to patients with Type-2 diabetes in Jordan.

The findings of this study have provided valuable insights into the factors affecting the management of Type- 2 diabetes in Jordan. The following section draws on the findings of this study and discusses the relevance and recommendations for patients, HCWs and managers, the wider community, policymakers and the research community. The relevance of the study for patients is discussed below.

\subsubsection{Relevance and recommendations for patients}

This study enabled the identification of a number of important implications for patients with poorly controlled Type-2 diabetes. This study allowed patients with high BG levels who are in the greatest need to express their concerns and needs which have not previously been investigated in Jordan. This is previously undocumented in the Jordanian literature due to few qualitative studies having been conducted. Therefore, this study provides new knowledge and helps to address the shortfall in the literature, especially in Jordan. This study also provides insight into patients' experiences and barriers to the management of diabetes which may be utilised to improve their management plan.

The findings from this study may be used to inform the development of an educational programme for patients with Type- 2 diabetes that reflects the expressed needs of patients as identified from the collected data. Patients believed health education to be an effective tool in 
empowering them to take responsibility for their own condition. Content which patients wanted to be included in a future educational programme included diabetes complications, herbal remedies, stressful factors, the emotional component of food and diabetes management behaviours (diet, physical activity, SMBG and medications). A group discussion was the preferred health education format. Group discussion encouraged patients to share and reveal their concerns and feelings with other patients. The group discussion provided a good opportunity for patients to speak and listen to each other, exchange knowledge and help each other. Focus groups are an inclusive method as anyone who can talk is eligible to participate so this helps to address any literacy issues.

However, through my observations, I noted that health education was only delivered through individual, face to face method when the patients visited the doctor on a monthly basis or when the doctor referred patients to the dietitian.

Recommendations for patients are that:

- Patients are involved in the design and delivery of an educational programme

- Patients are given the opportunity to have their opinions heard and discuss the challenges of living with diabetes

- Patients' accounts regarding the management of diabetes suggested that influences on their diabetes management occurred at multiple levels. Therefore, patients should be encouraged to talk about challenges that hinder their adherence to any management plan during consultations with HCWs, especially physicians. This information will be used to inform the development of an educational programme that accommodates patients' expressed needs, preferences and circumstances.

In addition to the relevance to patients, this study's findings also have relevance and recommendations for HCWs and managers which are now discussed.

\subsubsection{Relevance and recommendations for health care workers and managers}

This study identified many challenges and facilitators to patients' adherence to their diabetes management plan. These findings may be utilised to enhance the roles of physicians, pharmacists and nurses which then, in turn, may improve the management of Type-2 diabetes. This study's findings can also inform the development of an educational programme for patients with Type- 2 diabetes that could be used by HCWs to educate patients. 
A key finding from this study is that health promotion was not prioritised by the doctors, nurses and the pharmacists because of a lack of time and workload. There was a lack of patient-HCW communication in respect to health education mainly due to staff workload and time pressures and HCWs did not have active roles in conducting health education sessions in the health care centre. Most of the HCWs were not involved in health promotion initiatives. The physicians' role was mainly limited to prescribing medications, whereas the nurses' role was limited to taking vital signs and the pharmacists' role was dispensing medications. These findings can aid developments in HCWs' practice by valuing health promotion and taking social factors into account that enhances patients' self-management of diabetes.

This study's findings suggest that medical and nursing practice could usefully move away from a task-oriented medical model towards a more holistic approach in diabetes management (see figure 3). Displaying posters and utilising leaflets which patients wanted would also raise awareness and knowledge regarding diabetes in the health care centre.

The importance of factors beyond the individual level in terms of social, cultural, organisational and policy influences such as food habits, national health insurance policies and lack of continuity of care were identified as key factors which influence adherence to the diabetes management plan. Recognition of multiple determinants of patients' health among HCWs may expand the scope of interventions to seek to modify social norms and values. HCWs are also encouraged to be equipped with information about the influence of socio-cultural factors and patients' fatalistic beliefs related to religion and God in order to understand patients' health behaviours. HCWs may then be able to provide patients with appropriate health advice that is consistent with patients' beliefs and values. For example, most of the participants talked about health care seeking behaviours and their experiences with herbal remedies. HCWs did not routinely explore patients' use of herbs when taking patients' histories and prescribing their medications which could influence their glycaemic control.

Doctors and nurses may encourage patients to express their beliefs and concerns regarding their condition. Stressful life events upon diagnosis and positive family history were mentioned by most of the patients as causes of diabetes and attributed to their high BG levels. Misconceptions about medications were also expressed by patients in this study. Adherence to the medication regimen was affected by experiencing side effects and information received from other sources which included family and friends. Patients need to be provided with accurate information about medications, side effects and therapeutic effects. An educational programme may rectify 
misconceptions about medications especially, Metformin and insulin among patients. Doctors and nurses may also explore patients' needs, views, beliefs and perceptions about their condition in an open and approachable way.

My study findings suggested that using the actual Arabic term of medications (الأدوية) among HCWs instead of treatment (علاج) may help to rectify patients' misconceptions of medications. In the previous educational programme and during data collection, I noticed that most HCWs and patients used the term treatment in Arabic (علاج) to refer to medications. Therefore, the patient or family carer may perceive that medications can cure diabetes and indeed six patients did refer to diabetes as being curable.

The health centre's policy could usefully be reviewed to allow for patients to have three months' supply of medications instead of one month which may reduce work pressure on the staff, feelings of medication insecurity among patients and reduce travel costs incurred by patients. A different system could also be used so that the patient may see the pharmacist every month at the medication refills and then see the doctor every three months for monitoring the patient's progress similar to the Repeat Dispensing Policy in the UK (NHS, 2013).

Discontinuity of care was one of the main issues identified by most patients and HCWs in this study. Seeing the same doctor was suggested by approximately half of the patients as a facilitator to promote continuity of care and improve their experience in receiving care in the centre.

Recommendations for HCWs and managers are that:

- The skills and knowledge of existing HCWs, such as the nurse and dietician who have been trained to deliver the health education sessions could usefully be drawn upon to provide more health education (see figure 3 ).

- A holistic approach incorporating patient-centred care could usefully be incorporated into educational programmes to improve the understanding of patients' health and information needs.

- Herbal use is routinely discussed in taking the patient's medical history.

- The centre policies could usefully be reviewed to give the patients the opportunity to see the same doctor as they may feel comfortable and safe being treated by a familiar doctor who also knows them (see figure 3 ). 
- Reviewing the current appointment system in the health care centre may also be helpful for improving continuity of care and patients' satisfaction (see figure 3).

- Including family members in educational interventions may provide support to patients, enable the development of healthy home eating habits and promote diabetes selfmanagement.

- The application of the SEM (McLeroy et al., 1988) may inform understanding of the reasons behind high $\mathrm{BG}$ levels among patients which then, in turn, may improve the management of diabetes.

This study also revealed a number of socio-cultural factors that affected patients' health decisions. The relevance and recommendations for the wider community to facilitate the development of a health-enabling environment are presented now.

\subsubsection{Relevance and recommendations for the wider community}

This study allowed patients' family carers to express their concerns which have not previously been investigated in Jordan. This study identified a number of contextual factors that affected patients' adherence to their diabetes management plan. These factors highlighted the overwhelming influence of socio-cultural factors as determinants of patients' health and health behaviours. Patients talked about the influence of family, friends and culture on their food preferences and food choices that they made on a daily basis and during social gatherings. Information about diabetes in Jordan needs to be provided to the public and especially patients' family members who are at risk of developing diabetes due to a strong family history of the disease.

Provision health education only to patients could limit its impact on them as family members influenced patients' management plan, especially food choices. The family members' perceptions are necessary to take into account when developing educational programmes for patients. Patients also suggested other settings such as schools, universities and mosques could be utilised to raise awareness of Type- 2 diabetes and the risk factors among the general population in Jordan.

Increased awareness may mitigate against a community perception that eating high-fat, highsugar food is not harmful to patients with diabetes to seek to reduce the social pressure on patients to eat such foods from the broader community. Such awareness will enable the 
Jordanian community to provide social support to its members and raise awareness of diabetes amongst the wider community and population in Jordan.

Recommendations for the wider community are that:

- Improved eating habits by the Jordanian population and increasing the nutritional knowledge of family carers may positively influence the foods that patients consumed and may also impact on reducing the levels of obesity and associated risks of developing Type2 diabetes.

- Health education should be delivered in a wider context to target the population in different age groups to raise awareness of diabetes amongst the general population in Jordan.

- Introducing national health problems such as diabetes into schools may create more awareness in future generations that may enhance the role of family carers in patient's management of diabetes and hopefully reduce the prevalence of Type- 2 diabetes as the next generation adopt healthier lifestyles.

In addition to having relevance and recommendations to the wider community, these study findings also have relevance and recommendations for policymakers.

\subsubsection{Relevance and recommendations for policymakers}

Findings from this study identified gaps in the national health policy and health promotion programmes related to diabetes. Participants suggested a number of facilitators to diabetes prevention and management at the national health care system level in Jordan. The national health insurance schemes and medical exemption policies were identified by patients as barriers that hindered their adherence to their management plan, especially medications supply. The health insurance policies need to be reviewed to minimise medication wastage, overdosing and improve patient's follow up. Therefore, patients will not have to come to the health care centre on multiple trips to receive all their prescribed medications.

The main challenges related to medications that were expressed by patients were medication shortages and changing medications brands. I have to acknowledge that there may be cost issues as to why brands are changed. However, the frequent changes of brands impacted negatively on medication adherence among patients as evidenced by the study findings.

Patients' accounts identified many barriers that were influenced by socio-cultural norms. Lack of an exercise culture, lack of designated areas for exercise where both men and women have 
designated spaces, and social norms such as the idea of mixed-sex health clubs is restricted which affected patients' efforts to engage in physical activity. Public health policies need to address these barriers and establish appropriate strategies to address these barriers.

The proactive screening approach could be utilised as a prevention strategy to identify and manage undiagnosed diabetes among patients. Most of the patients in this study reported to the health facility seeking medical help after diabetes had been detected, and its complications had already developed (section 5.2). Two-thirds of the patients $(n=26)$ had a positive family history in this study. Therefore, the health care system in Jordan could usefully offer alternative approaches such as more proactive approaches to assess and detect health problems among the population at high risk of diabetes. For example, screening people with a positive family history to detect unknown cases of diabetes among them. Therefore, family history could be used as an indicator for detecting diabetes early among people. Type- 2 diabetes has a long preclinical phase, and it can be detected by screening programmes (ADA, 2017) through the primary health care centres.

Multiple trips, availability of family carers to accompany the patients (older and sick patients), availability of the tests, lack of an appointment system and the time factor were factors that affected doing the laboratory tests among the patients. It is written in the National Strategic Plan (2011-2012) that all laboratories in the comprehensive health care centres would be at least equipped with the HbA1c test over six years ago. However, the HbA1c test and other essential laboratory tests such as postprandial blood glucose test, lipids profile tests and liver enzymes were not available in the selected centre during the period of this study. The health centre is located a short distance away from most of the patients' residences. Provision of these tests at the centre would also mean that HCWs would have accessibility to accurate and complete records of laboratory test results for patients.

Recommendations for the policymakers are that:

- The national health insurance policies could also usefully be reviewed to give the patients the option to receive their medications from private pharmacies without paying extra money for their medications (see figure 3).

- When the medication brands are changed, patients are fully informed of any changes in tablet size or colour and reassured about the efficacy of the medication. 
- More culturally sensitive, gender-specific health and fitness clubs are needed which are affordable.

- Public health campaigns are needed such as through the mass media to raise awareness of the risk factors associated with diabetes to seek to reduce the prevalence of diabetes.

- The provision of diabetes specialist nurses could expand the roles of the nurses at the health care centre to provide health education which in turn may reduce the workload on doctors, improve continuity of care and provide the patient with an opportunity to discuss other health needs with nurses.

- Provision of the essential laboratory tests at the centre would reduce travel expenses and time and may encourage patients to attend regularly for testing as requested (see figure 3).

- Introducing an appointment system in the central laboratory may also be helpful for improving patients' satisfaction and reducing the patients' waiting time to be seen by lab technicians.

- $\quad$ Providing funding to deliver sustainable health education programmes in all comprehensive primary care settings.

In the next section, I present the relevance and recommendations for the research community as identified by this study.

\subsubsection{Relevance and recommendations for the research community}

This study identified a number of important findings, but it also generated a number of areas for future research.

The findings identified the factors that hindered patients' adherence to diabetes management behaviours. Most of these factors were influenced by the socio-cultural context, food habits and misconceptions about the management of diabetes. The prevalence of these factors and the extent to which they influence diabetes management can be further validated in a larger sample that represents the whole of Jordan.

Recommendations for the research community are that:

- There is a need for further studies to explore patient-HCW communication and the role of patients in the management plan. Further ethnographic and qualitative studies that include detailed observations of consultation sessions would provide an in-depth understanding of 
patient-HCW communication and relationships which may influence adherence to the diabetes management plan. Findings from such studies coupled with findings from my study can then be utilised to inform policies and practice that will improve patient-HCW communication and may aid patients' satisfaction with their care.

- Further research could usefully explore the factors influencing the development and expansion of nurses' and pharmacists' roles in primary care and health promotion in Jordan.

- As I discussed in the literature, most of the existed literature used quantitative methods, and a cross-sectional survey design has dominated most of the published Jordanian literature. There is a need for more qualitative studies and public health research to go beyond quantitative research to identify and gain a more in-depth understanding of the issues and health needs from the perspectives of patients and their carers.

This ethnographic study aimed to identify factors associated with poorly controlled Type-2 diabetes in Jordan. This study was able to identify a number of factors affecting the management of Type-2 diabetes. Therefore, the qualitative findings from this study have contributed to the body of knowledge related to the management of Type- 2 diabetes in Jordan.

This study provides valuable new information for understanding patients' behaviours and attitudes towards management of diabetes in Jordan. It also illustrates how a patient's health was affected by multiple levels of influence rather than at one level. To be effective, health promotion interventions and education need to be tailored to meet the individual, social and cultural needs of patients. A patient-centred approach and a holistic approach are recommended in order to address different factors affecting patients' adherence to the management plan. This study also provided an opportunity for patients to express their views and concerns about their condition. This ethnographic study has identified and explained the factors associated with poorly controlled Type- 2 diabetes in one setting. Therefore, the aims and objectives of this study were achieved. However, as mentioned in the recommendations, further research is needed to develop an understanding of the factors affecting the management of diabetes within the wider context of Jordan. 


\section{Appendix 1. Research Ethics Committee approval letter.}

Swansea University Prifysgol Abertawe

18 May 2016

Ref: 080316

Amer Sahour

Postgraduate Student

Swansea University

Dear Amer

We would like to confirm that your research application entitled: An ethnographic study of the factors associated with poor glycaemic control levels among people with type 2 diabetes accessing Comprehensive Health Centres in Jordan was reviewed by members of the CHHS and CoM Research Ethics Committee on 7 March 2016 and was given approval on 10 March 2016 .

\section{Regards,}

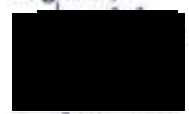

Dr. J.L.Hewitt

Chair, CHHS and College of Medicine Research Ethics Committee www.chhsresearchethics.swan.ac.uk

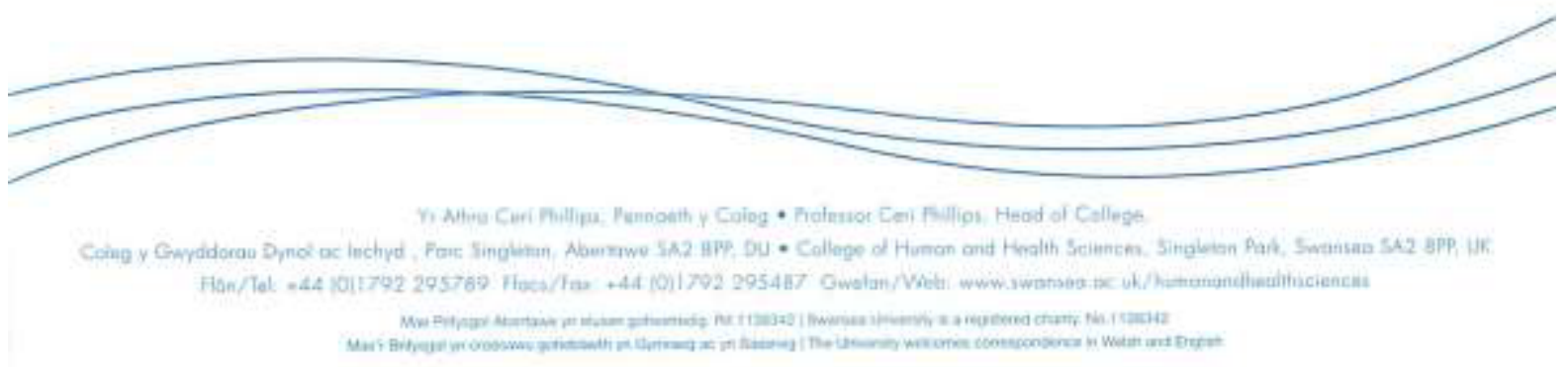




\section{Appendix 2. The Ministry of Health ethical approval letter}
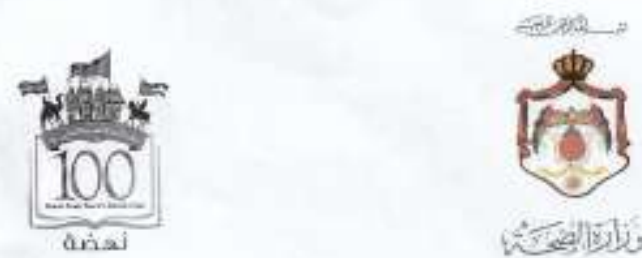

Af

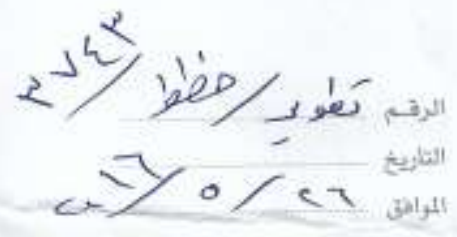

مدير صحة محافظة

$$
\text { تحمية طيبة وبعد ف6 }
$$

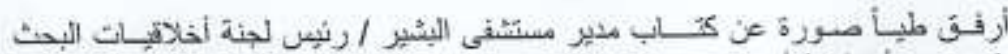

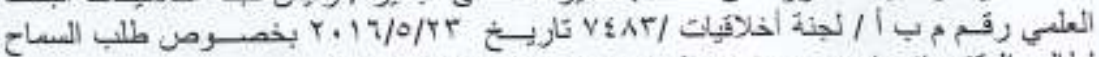

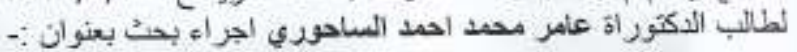

(An ethnographic study of the factors associated with poor glycaemic control levels among people with type 2 diabetes accessing a primary care centre in Jordan)

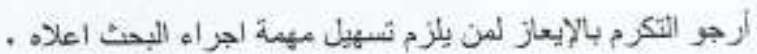

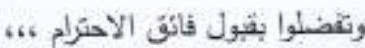
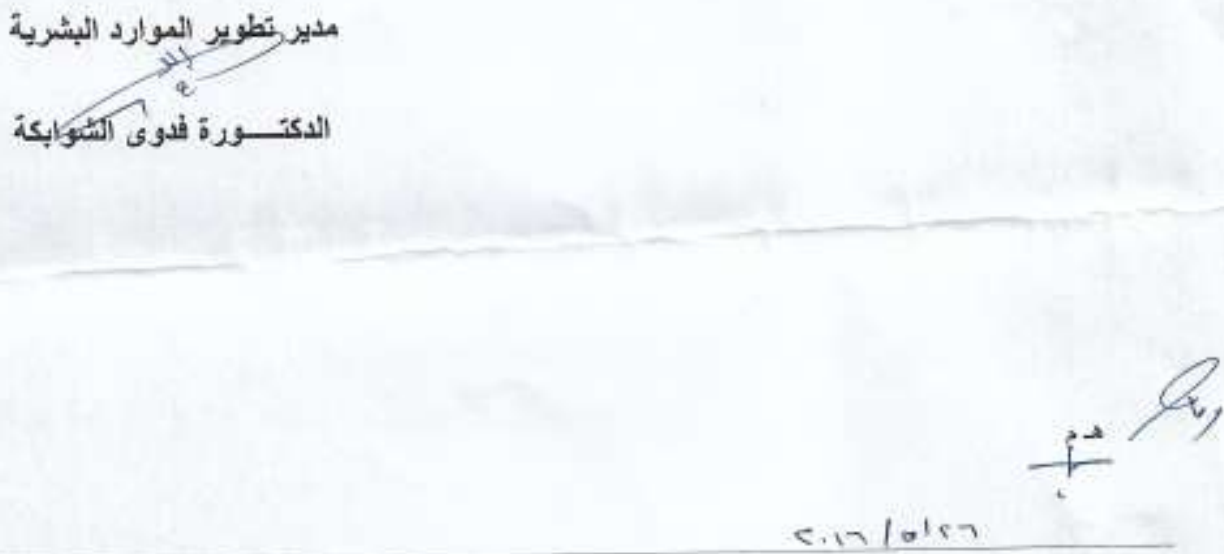

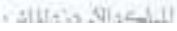




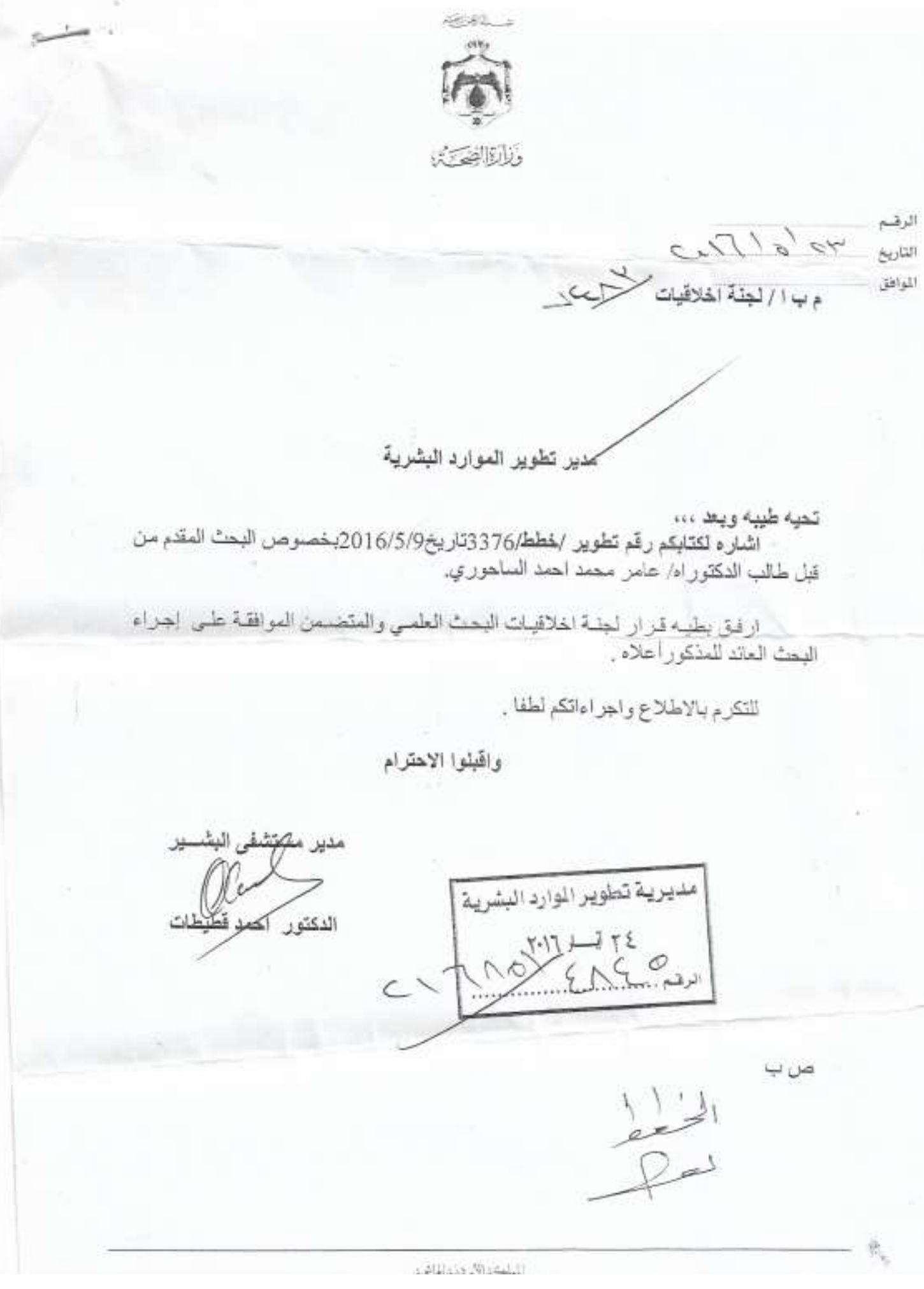




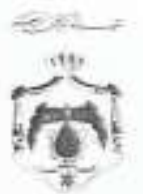

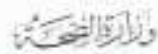

CODE : MOH REC 160080

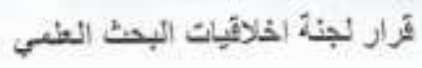

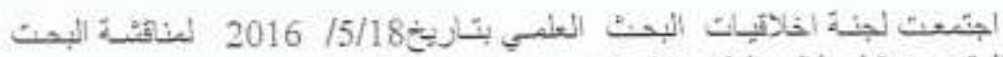

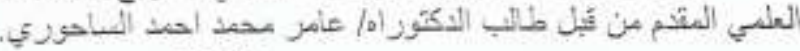

$$
\begin{aligned}
& \text { بثلئن }
\end{aligned}
$$

(An ethnographic study of the factors associated with poor glycaemic control levels among people with type 2 diabetes accessing aprimary care centre in Jordan)

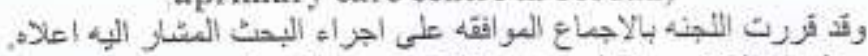

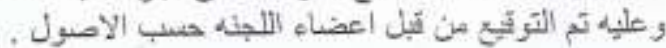

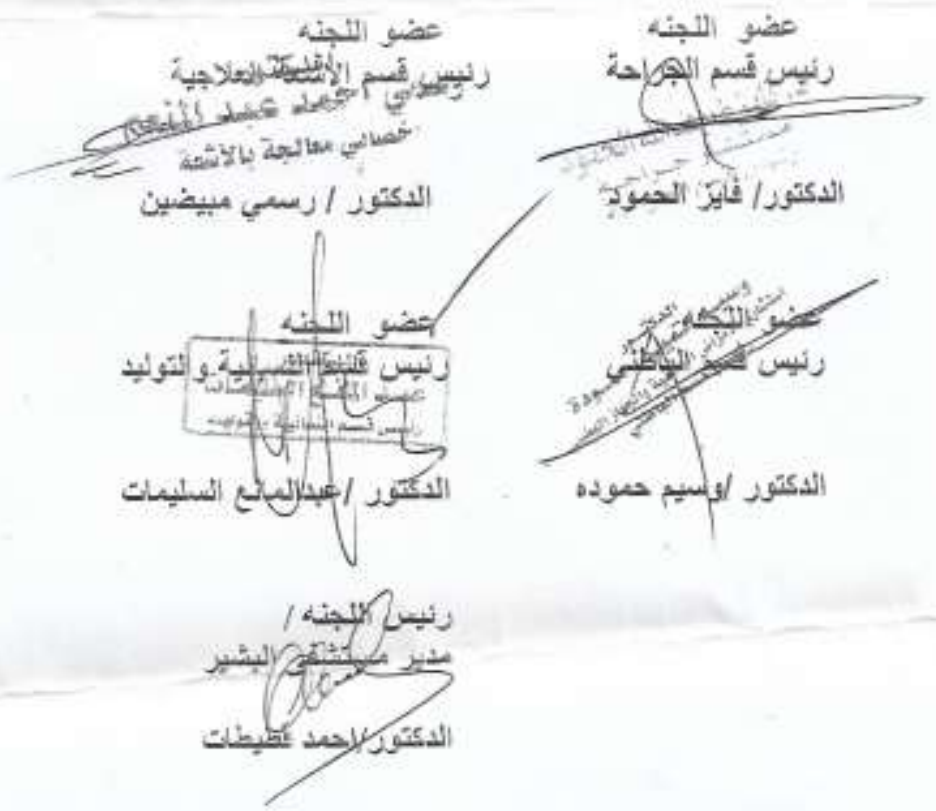

عضو اللجنبة

المصنامدانتمريض

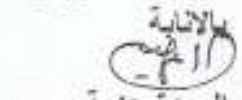

السبئi

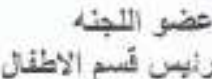

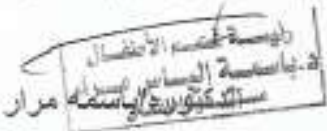

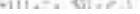



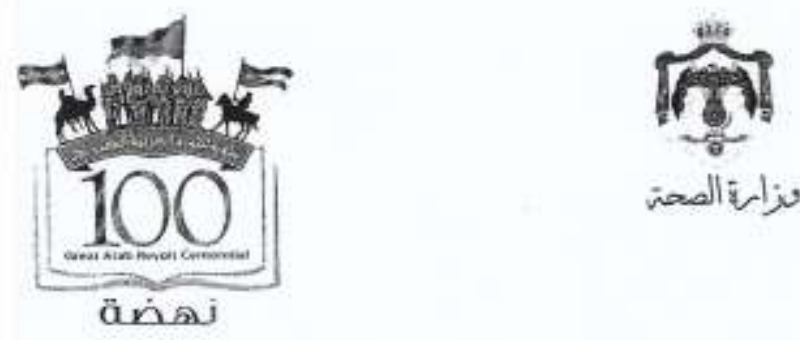

$$
\text { radicio }
$$

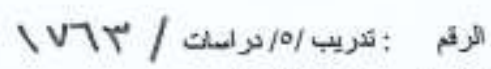
r.IY/V/CE: التزاريخ

\footnotetext{
رئيس مركز صحي ............................
}

$$
\text { ت،رية طيية وبعد }
$$

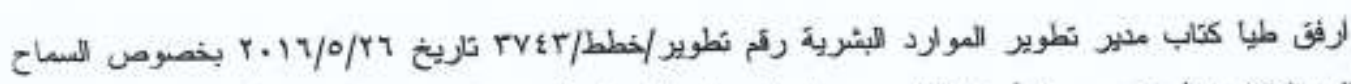

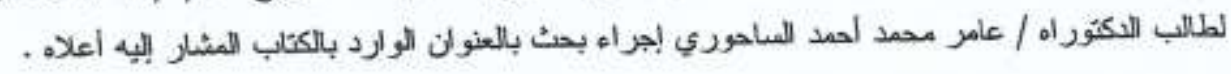

$$
\begin{aligned}
& \text { لبستهيل مهيمة إجز اء البيحث . }
\end{aligned}
$$

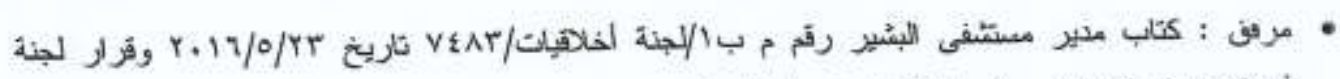

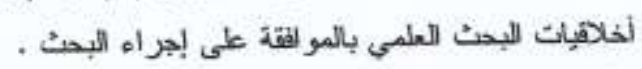

$$
\text { وتفضلو بقيول فائق الاحتر ام ،،، ، }
$$

\section{| مدير صحة محافظة .}

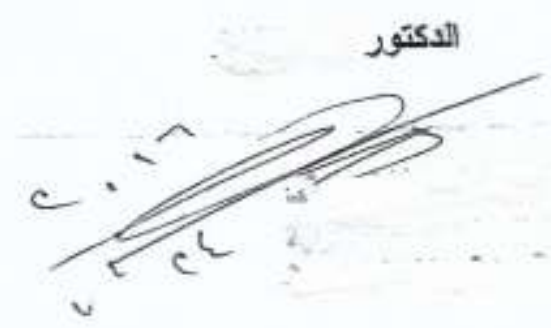

نسخة / العساء للر عاية المحية الاولية

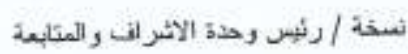
نسخة / الملف الت ستة / لمتسلسلة $r+17 / 3 / 40 .+2$ 


\section{Appendix 4. Invitation letter to potential group discussion participants}

Dear Sir/ Madam

I am Amer Al-Sahouri and I am a PhD student in Public Health at Swansea University, Wales, UK. I am conducting research about exploring the factors associated with poor blood sugar levels among a sample of people with type- 2 diabetes accessing primary care centres in Jordan. With your permission I would like to invite you to participate in a group discussion.

You have been chosen for this study because you have type- 2 diabetes and your blood sugar level is not within normal limits. Patients with type-2 diabetes are in a prime position to talk about their experiences and views regarding type- 2 diabetes and the factors which hinder and help in maintaining blood sugar levels within normal limits.

The group discussion will be conducted in a private room in the primary care centre or another mutually convenient place. The group discussion will last approximately 90 minutes and the discussion will take part between you, myself, a FG assistant and approximately 6 other participants who also have type-2 diabetes with a blood sugar level which is not within normal limits. You will receive glucometer which is equivalent to 12 Jordanian Dinar (approximately 12 pounds sterling) as a token of appreciation for your time and assisting you with the management of your diabetes. It will be your choice whether to take part or not and your care will not be affected whatever choice you make. I have attached a reply slip and an information sheet with more details about the study. Please contact me if you need more information. If you agree to participate, I will contact you to arrange the date, time and venue for the group discussion session. Even if you agree to participate, you can withdraw at any time without giving a reason.

Regards,

Amer Al-Sahouri

PhD student in Public Health at the Swansea University, Wales, UK. 


\section{Appendix 5. Patient reply slip}

\section{(Group discussion)}

\section{Please complete this slip and deposit back in the box in the waiting area}

I am interested in participating in this study (please tick)

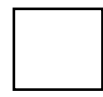

If you are interested in this study and would like to hear more, I will contact you once you have returned this reply slip. I will give more information about the study, date, venue and time of the group discussion. Please write your contact details below.

Contact details

Name:

Telephone number at home/ work (if appropriate):

Mobile number:

Email address:

I would prefer you to contact me (please tick):

Morning

Afternoon

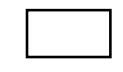

Evening

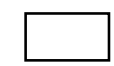

Any preferred days

Thank you for showing interest in this research. 


\section{Appendix 6. Participant information sheet}

\section{(Group discussion)}

\section{Invitation statement:}

You are being asked to take part in a research study to explore the factors associated with poor blood sugar levels among a sample of people with type-2 diabetes accessing primary care centers in Jordan.

\section{Who is the researcher?}

My name is Amer Al-Sahouri and I am a PhD student in Public Health at Swansea University, Wales, UK. I have completed a Bachelor's degree in Nursing and a Master's degree in Public Health. I have three years' experience in critical care nursing and five years' experience in teaching medical and nursing courses to nursing students, health care providers and non-health care providers.

\section{Why have you been chosen?}

You have been chosen for this study because you have type-2 diabetes and your blood sugar level is not within normal limits. Patients with type-2 diabetes are in a prime position to talk about their experiences and views regarding type- 2 diabetes and the factors which hinder and help in maintaining blood sugar levels within normal limits. Your participation in this study will help me and other researchers understand the factors that may improve diabetes management plans and help with preventing poorly controlled type-2 diabetes in Jordan in the future.

\section{Do you have to take part?}

No. It will be your choice to participate. If you have read this information sheet and are willing to participate, I will ask you to complete a short questionnaire about yourself which will not identify you by name. Before the group discussion starts, you will also be asked to sign a consent form and even if you decide to take part you still have the right to refuse to answer any question or withdraw at any time and without giving a reason. Any data collected from you will be destroyed if you decide to withdraw from this study. Whether you are happy to participate in this study or not, that will not affect your care. 


\section{What is the research about?}

This study seeks to explore the reasons why maintaining blood sugar levels within normal limits of patients with type 2 diabetes is poor in Jordan. It will encompass an exploration of the perceptions and experiences of patients with type 2 diabetes, their family carers and health care workers regarding the barriers and facilitators to maintaining blood sugar levels within normal limits. The findings of this study will be used to inform policy and practice and specifically will be used to inform the development of an educational package for patients with type 2 diabetes in Jordan.

\section{What will you have to do in this study?}

You will be asked to participate in the group discussion that will take place between you, myself, a FG assistant and approximately 6 other participants who also have type-2 diabetes with a blood sugar level which is not within normal limits. You will be asked to talk about your experiences and views regarding type- 2 diabetes and the factors which hinder and help in maintaining blood sugar levels within normal limits. There are no wrong or right answers

This discussion will last approximately 90 minutes and I will arrange the place and time convenient for you. The group discussion can be conducted in a private room in the selected primary care centre or another mutually convenient place. I would like to audio record the group discussion and this will be done only with your permission.

\section{Will your participation in this study be kept confidential?}

Yes. Before beginning the group discussion ground rules will be established to seek to preserve the confidentiality of the discussion between participants and all participants will be asked to follow these rules. Additionally, if the audiotape is used I will be the only person who listens to the tape. When I write my thesis and reports, I will use pseudonyms for all names in order to protect your identity. Audiotapes, if used, will be destroyed 5 years after completion of the study.

\section{Has the researcher had permission to conduct this research?}

Yes. I have had permission from the director of the selected primary care centre. Additionally, the research has gained ethical approval from the College of Human and Health Sciences Ethics 
Committee at Swansea University in United Kingdom and from the Ministry of Health in Jordan.

\section{Will you receive any incentive?}

Yes. You will receive glucometer which is equivalent to 12 Jordanian Dinar (approximately 12 pounds sterling) as a token of appreciation for your time and assisting you with the management of your diabetes.

\section{What will happen to the findings of this study?}

This study will be written up and submitted as my $\mathrm{PhD}$ thesis to Swansea University. The findings of my research will be published as articles in academic journals and presented as research papers at conferences. You will not be identifiable in any findings, reports or papers.

\section{What do you receive from the study?}

Once the research has been completed, I will be pleased to send you a summary of the research findings by email or post.

\section{How can I contact you for further information?}

Please contact me if you have any queries or you need more information. My contact details are given below. If you agree to participate in this study, I will contact you to arrange the date, time and venue of the group discussion.

Thank you for your time.

Amer Al-Sahouri.

College of Human and Health Sciences

Swansea University

E-mail

Mobile Jordan: 


\section{Appendix 7. Participant information sheet}

\section{(Health care workers)}

\section{Invitation statement:}

You are being asked to take part in a research study to explore the factors associated with poor blood sugar levels among a sample of people with type-2 diabetes accessing primary care centres in Jordan.

\section{Who am I?}

My name is Amer Al-Sahouri and I am a PhD student in Public Health at Swansea University, Wales, UK. I have completed a Bachelor's degree in Nursing and a Master's degree in Public Health. I have three years' experience in critical care nursing and five years' experience in teaching medical and nursing courses to nursing students, health care providers and non-health care providers.

\section{Why have you been chosen?}

You are being invited to participate in this study because you are a health care worker involved with delivering care to people with type-2 diabetes. Health care workers are important sources of information about patients' health needs and factors affecting adherence to their treatment plans. Health care workers such as nurses, doctors, pharmacists, reception staff and dietitian are part of multidisciplinary team of type- 2 diabetes management. Your participation in this study will help me and other researchers understand the factors that may improve diabetes management plans and help with preventing poorly controlled type- 2 diabetes in Jordan in the future.

\section{Do you have to take part?}

No. It will be your choice to be interviewed. If you have read this information sheet and are willing to participate you will be asked to sign a consent form and even if you decide to take part you have the right to refuse to answer to any question or withdraw at any time and without giving a reason. Any data collected from you will be destroyed if you decide to withdraw from this study. 


\section{What is the research about?}

This study seeks to explore the reasons why maintaining blood sugar levels within normal limits of patients with type 2 diabetes is poor in Jordan. It will encompass an exploration of the views and experiences of patients with type 2 diabetes, their family carers and health care workers regarding the barriers and facilitators to maintaining blood sugar levels within normal limits. The findings of this study will be used to inform policy and practice and specifically will be used to inform the development of an educational package for patients with type 2 diabetes in Jordan.

\section{What will you have to do in this study?}

You will be asked to be interviewed by me. You will be also asked to talk about your views in relation to type 2 diabetes and the barriers and facilitators to maintaining blood sugar levels within normal limits. There are no wrong or right answers. This interview will last approximately 45 minutes and can be conducted in a private room in the selected primary care centre or another mutually convenient place. I would like to audio record the interview and this will be done only with your permission.

\section{Will your participation in this study be kept confidential?}

Yes. I guarantee your views will be kept confidential. If the audio recorder is used I will be the only person who listens to the tape. When I write my thesis and reports, I will use pseudonyms for all names in order to protect your identity. Audiotapes, if used, will be destroyed 5 years after completion of the study.

\section{Have I had permission to conduct this research?}

Yes. I have had permission from the director of the selected primary care centre. Additionally, the research has gained ethical approval from the College of Human and Health Sciences Ethics Committee at Swansea University in United Kingdom and from the Ministry of Health in Jordan.

\section{What will happen to the findings of this study?}

This study will be written up and submitted as my $\mathrm{PhD}$ thesis to Swansea University. The findings of my research will be published as articles in academic journals and presented as research papers at conferences. You will not be identifiable in any findings, reports or papers. 


\section{What do you receive from the study?}

Once the research has been completed, I will be pleased to send you a summary of the research findings by email or post.

\section{How can you contact you for further information?}

Please contact me if you have any queries or you need more information. My contact details are given below. If you agree to participate in this study, I will contact you to arrange the time and venue of the interview.

Thank you for your time.

Amer Al-Sahouri.

College of Human and Health Sciences

Swansea University

E-mail:

Mobile Jordan: 


\section{Appendix 8. Participant information sheet}

\section{(Family carers)}

\section{Invitation statement:}

You are being asked to take part in a research study to explore the factors associated with poor blood sugar levels among a sample of people with type-2 diabetes accessing primary care centres in Jordan.

\section{Who am I?}

My name is Amer Al-Sahouri and I am a PhD student in Public Health at Swansea University, Wales, UK. I have completed a Bachelor's degree in Nursing and a Master's degree in Public Health. I have three years' experience in critical care nursing and five years' experience in teaching medical and nursing courses to nursing students, health care providers and non-health care providers

\section{Why have you been chosen?}

You are being invited to participate in this study because you are a family carer of a relative with type-2 diabetes. Family carers are important sources of information about patients' health needs and factors which hinder and help in maintaining blood sugar levels within normal limits. Family carers such as relatives, family members and significant others are part of multidisciplinary team of type-2 diabetes management. Your participation in this study will help me and other researchers understand the factors that may improve diabetes management plans and help with preventing poorly controlled type-2 diabetes in Jordan in the future.

\section{Do you have to take part?}

No. It will be your choice to be interviewed. If you have read this information sheet and are willing to participate you will be asked to sign a consent form and even if you decide to take part you have the right to refuse to answer to any question or withdraw at any time and without giving a reason. Any data collected from you will be destroyed if you decide to withdraw from this study. 


\section{What is the research about?}

This study seeks to explore the reasons why maintaining blood sugar levels within normal limits of patients with type 2 diabetes is poor in Jordan. It will encompass an exploration of the views and experiences of patients with type 2 diabetes, their family carers and health care workers regarding the factors which hinder and help in maintaining blood sugar levels within normal limits. The findings of this study will be used to inform policy and practice and specifically will be used to inform the development of an educational package for patients with type 2 diabetes in Jordan.

\section{What will you have to do in this study?}

You will be asked to be interviewed by me. You will be also asked to talk about your views in relation to type 2 diabetes and factors which hinder and help in maintaining blood sugar levels within normal limits. There are no wrong or right answers. This interview will last approximately 45 minutes and can be conducted in a private room in the selected primary care centre or another mutually convenient place. I would like to audio record the interview and this will be done only with your permission.

\section{Will your participation in this study be kept confidential?}

Yes. I guarantee your views will be kept confidential. If the audio recorder is used I will be the only person who listens to the tape. When I write my thesis and reports, I will use pseudonyms for all names in order to protect your identity. Audiotapes, if used, will be destroyed 5 years after completion of the study.

\section{Have I had permission to conduct this research?}

Yes. I have had permission from the director of the selected primary care centre. Additionally, the research has gained ethical approval from the College of Human and Health Sciences Ethics Committee at Swansea University in United Kingdom and from the Ministry of Health in Jordan.

\section{What will happen to the findings of this study?}

This study will be written up and submitted as my $\mathrm{PhD}$ thesis to Swansea University. The findings of my research will be published as articles in academic journals and presented as research papers at conferences. You will not be identifiable in any findings, reports or papers. 


\section{What do you receive from the study?}

Once the research has been completed, I will be pleased to send you a summary of the research findings by email or post.

\section{How can you contact you for further information?}

Please contact me if you have any queries or you need more information. My contact details are given below. If you agree to participate in this study, I will contact you to arrange the time and venue of the interview.

Thank you for your time.

Amer Al-Sahouri.

College of Human and Health Sciences

Swansea University

E-mail:

Mobile Jordan: 


\section{Appendix 9. Participant consent form}

Full title of proposal: A study of the factors associated with poor blood sugar levels among a sample of people with type- 2 diabetes accessing primary care centers in Jordan.

Name of researcher: Amer Al-Sahouri

Position: PhD student in Public health in Swansea University

Purpose of data collection: $\mathrm{PhD}$ dissertation

\section{Please tick the appropriate box}

\begin{tabular}{|l|l|}
\hline Statements of consent & Box \\
\hline I confirm that I have read and understood the information sheet dated DD/MM/YYYY for this \\
research. \\
\hline I have had the opportunity to consider the participant information sheet, ask questions and my \\
questions have been answered to my satisfaction. \\
\hline $\begin{array}{l}\text { I understand that my participation is voluntary which I can withdraw from the above study at any time } \\
\text { without giving reason, and if I choose to withdraw, my data will be removed from the study and will } \\
\text { be not used. }\end{array}$ \\
\hline $\begin{array}{l}\text { I am assured that if I agree to the group discussion or the interview being audio-recorded that only } \\
\text { Amer Al-Sahouri will hear the recording and that he will do the transcription himself using } \\
\text { pseudonyms to protect my identity. }\end{array}$ \\
\hline $\begin{array}{l}\text { I have been guaranteed that it will not be possible to discover my identity from the written thesis, } \\
\text { reports or publications. }\end{array}$ \\
\hline $\begin{array}{l}\text { I have been guaranteed that my data will be held confidentially, stored in a locked cabinet in a locked } \\
\text { office and only Amer Al-Sahouri will have access to them. }\end{array}$ \\
\hline $\begin{array}{l}\text { I agree that the overall findings may be submitted for publication in a scientific journal, or presented } \\
\text { at scientific conferences. }\end{array}$
\end{tabular}




\section{$\underline{\text { Name, signature and date }}$}

\section{Participant:}

Name of participant

Date-

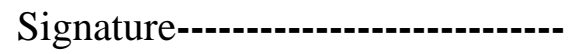

\section{Researcher:}

Name of researcher

Date-

Signature 


\section{Appendix 10. Group discussion topic guide}

\section{Preliminary activities}

-Greet participants.

-Answer any questions related to participant information sheet.

-Give the participants the consent forms to read and sign.

-Distribute and then collect the completed sociodemographic questionnaires from participants.

\section{Introduction}

-Overview of research aim.

-Discussion of format of the group discussion and that the discussion will last approximately 90 minutes.

-Establish the ground rules to seek to preserve the confidentiality of the discussion.

-Agreement to use the audio recorder and the right to withdraw any time.

-No right or wrong answers.

-One person speaks at a time.

\section{Group discussion questions guide}

Knowledge of type-2 diabetes and self-care management

-What do you know about type-2 diabetes?

-How do you think you got diabetes?

Attitudes towards type-2 diabetes 
-How do you feel about diabetes?

-How do you feel about the support that you have received to help you to manage your diabetes?

Probe: by health care system?

By health care workers?

By family and friends?

\section{Behaviours toward type-2 diabetes}

-What kind of behaviours are you undertaking to take care of your diabetes?

Probe: healthy diet, physical activity, testing blood glucose levels and taking the recommended diabetes medications

-What do you think will happen if you do not manage your diabetes?

$\underline{\text { Barriers toward type-2 diabetes care }}$

-What are the barriers that you face when you are trying to take care of your diabetes?

Facilitators toward type-2 diabetes care

-What are the factors that help you to manage your diabetes?

$\underline{\text { Source of information about diabetes }}$ 
- Where do you get information about your diabetes from?

-What do you think about the information you receive?

Probe: easy to understand or not?

Educational package for people with type-2 diabetes

-What specific topics do you think are important for patients trying to manage their diabetes?

-When you get information about diabetes, what sort of format do you prefer that information is given to you in? Why?

-Can you tell me examples of formats that you prefer?

Probe: poster, face to face, group discussion, DVD, website and brochures.

-Do you think the primary care centre is a good place to attend for educational programmes or not? Other preferred places?

\section{Closing questions}

-Is there anything that you would like to add or ask?

-May I contact you again if there is any information that I want to clarify?

\section{Debriefing session}

A debriefing session will be conducted after each group discussion, so study participants can express their reactions and understandings to the discussions in the group discussion. I will then give the participants their incentives.

Thank you for taking part in the group discussion session for today. 


\section{Appendix 11. Semi structured interview topic guide}

\section{(Health care workers)}

\section{Preliminary activities}

-Greet participant.

-Answer any questions related to participant information sheet.

-Give the participant the consent forms to read and sign.

\section{Introduction}

-Overview of research aim.

-Discussion of format of the semi-structured interview and the interview will last approximately 45 minutes.

-Explain the confidentiality: Pseudonyms, only me will hear the recording.

-Agreement to use the audio recorder and the right to withdraw any time.

-No right or wrong answers.

\section{General information}

-What is your role in this primary care centre?

-How long have you worked in this primary care centre?

-Did you attend any formal diabetes training? If not, then why?

\section{Semi structured questions guide}


$\underline{\text { Awareness of poor controlled type }-2 \text { diabetes }}$

-How significant is poorly controlled type-2 diabetes in Jordan?

- What do you think people with poor controlled type-2 diabetes need in Jordan?

- How do you feel when you provide care (or service) to people with poorly controlled type-2 diabetes?

Factors contribute to poor controlled type-2 diabetes

-What are the factors associated with poorly controlled type-2 diabetes in Jordan?

- What do you think are the barriers to good glycaemic control levels?

$\underline{\text { Facilitators to improve glycaemic levels }}$

-What are the facilitators to improve glycaemic control levels among people with poor glycaemic control?

-Do you think the existing treatment plan is sufficient to improve glycaemic levels? If not, then why not?

Educational package for people with type-2 diabetes

-What do you think about the current diabetes education programme in this centre?

-What information is important for people with poorly controlled type- 2 diabetes which should be included in the educational programme? 
- What sort of format do you think is suitable for people with type-2 diabetes? Why?

Probe: poster, face to face, group discussion, DVD, website and brochures

-Do you think the primary care centre is a good place to attend educational programmes or not? Other suitable places?

\section{Closing questions}

-Is there anything that you would like to add or ask?

-May I contact you again if there is any information that I want to clarify?

Thank you for taking part in the interview for today. 


\section{Appendix 12. Semi structured interview topic guide}

\section{(Family carers)}

\section{Preliminary activities}

-Greet participant.

-Answer any questions related to participant information sheet.

-Give the participant the consent forms to read and sign.

\section{Introduction}

-Overview of research aim.

-Discussion of format of the semi-structured interview and the interview will last approximately 45 minutes.

-Explain the confidentiality: use of pseudonyms, only me will hear the recording.

-Agreement to use the audio recorder and the right to withdraw any time.

-No right or wrong answers.

\section{General information}

-What is your relationship to the patient?

-How long have you provided care to the patient?

Semi structured questions guide

Role of family in management of type-2 diabetes 
-What do you see as your role?

-What is your experiences of living with a person with type-2 diabetes?

-Do you think involvement of family and friends in the management of type- 2 diabetes is important or not? Why?

- What special considerations do you need to take care of a person with type-2 diabetes?

-What are the challenges about caring and living with a person with type- 2 diabetes? Any changes of daily routine activities?

$\underline{\text { Factors which contribute to poor controlled type-2 diabetes }}$

-What are the factors associated with poorly controlled type-2 diabetes?

-What do you think are the barriers to maintaining good sugar levels?

$\underline{\text { Facilitators to improve glycaemic levels }}$

-What are the factors which help to improve blood sugar levels among people with type-2 diabetes?

\section{Educational package for people with typ-2 diabetes}

-Have you attended appointments with your relative? Was this helpful or not?

-Have you or your relative attended any educational programmes about type- 2 diabetes and its management? If so what are your views about the educational programme? 
-What information is important for people with poorly controlled type-2 diabetes which should be included in the educational programme?

- What sort of format do you think is suitable for people with type-2 diabetes? Why?

(Poster, face to face, group discussion, DVD, website and brochures)

-Do you think the primary care centre is a good place to attend for educational programmes or not? Other preferred places?

\section{$\underline{\text { Closing questions }}$}

-Is there anything that you would like to add or ask?

-May I contact you again if there is any information that I want to clarify?

Thank you for taking part in the interview for today. 


\section{Appendix 13. The sociodemographic data questionnaire}

Full title of proposal: A study of the factors associated with poor blood sugar levels among a sample of people with type- 2 diabetes accessing primary care centers in Jordan

Name of researcher: Amer Al-Sahouri

Position: PhD student in Public health in Swansea University

Purpose of data collection: $\mathrm{PhD}$ dissertation

Date:

Please, read and complete this questionnaire.

Please tick the appropriate box.

1-How old are you?

$$
\text { 40-50 years } \square \quad 51-60 \text { years } \square \quad 61-70 \text { years } \square \quad \geq 71 \text { years }
$$

2-What is your gender?

\section{Male $\square \quad$ Female}

3-What is your highest level of education you have completed?

$$
\text { No education } \square \quad \text { Primary } \square \quad \text { Secondary school } \square \quad \text { Diploma }
$$

University degree or higher

4-How long have you been diagnosed with type-2 diabetes?

$1-5$ years $\square 6-10$ years $\square \geq 10$ years

5-What type of treatment do you undertake to manage your type-2 diabetes?

Diet only $\square$ Oral hypoglycaemic tablets $\square$ Insulin

Insulin and oral (combined)

6-Do you have a family history of diabetes?

Yes $\square$ No $\square$

7-What is your smoking status? 
Current smoker $\square$ Past smoker $\square$ Nonsmoker

8-What is your body mass index (BMI)?

Height---------- (cm) Weight----------- $(\mathrm{kg})$ BMI------------ $\left(\mathrm{kg} / \mathrm{m}^{2}\right)$

-Do you consider your weight as being

Normal $\square$ Over weight $\square$ Obese $\square$ Do not know

9-What is your marital status?

Single $\square$ Married $\square$ Divorced $\square$ Widowed

10-What is your monthly income in Jordanian Dinar (JD)?

300-500 JD $\square$ 501-1000 JD $\square \quad \geq 1000$ JD

12-Have you attended a formal diabetes education programme in the last year?

No

If yes $\square$. How long was the programme?

And where was it held?

Thank you for completing this questionnaire. 


\section{Appendix 14. Debrief sheet}

Thank you for taking part in this research which is exploring factors associated with poor blood sugar levels among a sample of people with type- 2 diabetes accessing primary care services in Jordan.

The findings will be used to inform policy and practice and specifically will be used to inform the development of an educational package for patients with type- 2 diabetes in Jordan.

If you would like to receive a summary of the research findings, then please let me know and I can email you when the study is completed.

Again, thank you for participation and if you have any questions regarding this study, please contact me at the following contact details:

Amer Al-Sahouri.

College of Human and Health Sciences

Swansea University

E-mail:

Mobile Jordan: 


\section{By following a diabetes care plan, diabetes can be managed effectively.}

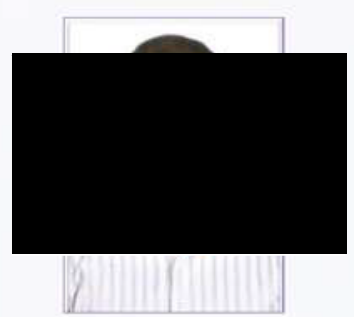

Full title of research: A study of factors associated with poor blood sugar levels among a sample of people with type-2 diabetes accessing primary care centers in Jordan.

My name is Amer Al-Sahouri and I am a PhD student in Public Health at Swansea University, Wales., UK.

As part of my PhD, I am pleased to let you know that participant observations are taking place in this centre from DD/MM/YYYY to DD/MM/YYYY.

I have had permission from the director of this primary care centre. Additionally, the research has gained ethical approval from the College of Human and Health Sciences Ethics Committee at Swansea University in United Kingdom and from the Ministry of Health in Jordan.

My contact details are :

Contact details:

E-mail:

Mobile Jordan:

Thank you for your time in reading this poster 
Appendix 16. General Information of the Focus Groups

\begin{tabular}{|c|c|c|c|c|c|c|}
\hline $\begin{array}{l}\text { Focus } \\
\text { group ID }\end{array}$ & $\begin{array}{l}\text { Number of } \\
\text { patients }\end{array}$ & Date & & emales & Location & Duration \\
\hline 1 & 6 & 27/07/2016 & 2 & 4 & $\begin{array}{l}\text { The } \\
\text { Comprehensive } \\
\text { Health Care } \\
\text { Centre. }\end{array}$ & 01:13:01 \\
\hline 2 & 3 & $28 / 07 / 2016$ & 3 & 0 & $\begin{array}{l}\text { The } \\
\text { Comprehensive } \\
\text { Health Care } \\
\text { Centre. }\end{array}$ & $01: 17: 41$ \\
\hline 3 & 5 & $09 / 08 / 2016$ & 2 & 3 & $\begin{array}{l}\text { The } \\
\text { Comprehensive } \\
\text { Health Care } \\
\text { Centre. }\end{array}$ & $01: 00: 28$ \\
\hline 4 & 6 & $11 / 08 / 2016$ & 3 & 3 & $\begin{array}{l}\text { The } \\
\text { Comprehensive } \\
\text { Health Care } \\
\text { Centre. }\end{array}$ & 1:08:58 \\
\hline 5 & 4 & $25 / 08 / 2016$ & 3 & 1 & $\begin{array}{l}\text { The } \\
\text { Comprehensive } \\
\text { Health Care } \\
\text { Centre. }\end{array}$ & $1: 07: 48$ \\
\hline 6 & 4 & $01 / 09 / 2016$ & 1 & 3 & $\begin{array}{l}\text { The } \\
\text { Comprehensive } \\
\text { Health Care } \\
\text { Centre. }\end{array}$ & $01: 31: 25$ \\
\hline 7 & 5 & 06/10/2016 & 1 & 4 & $\begin{array}{l}\text { The } \\
\text { Comprehensive } \\
\text { Health Care } \\
\text { Centre. }\end{array}$ & $55: 50$ \\
\hline
\end{tabular}




\begin{tabular}{|c|c|c|c|c|c|c|}
\hline 8 & 5 & $15 / 11 / 2016$ & 1 & 4 & $\begin{array}{l}\text { The } \\
\text { Comprehensive } \\
\text { Health Care } \\
\text { Centre. }\end{array}$ & $45: 59$ \\
\hline
\end{tabular}


Appendix 17. General Information of Health Care Workers who were interviewed in this study $(n=10)$

\begin{tabular}{|c|c|c|c|c|c|}
\hline $\begin{array}{l}\text { A number } \\
\text { of the } \\
\text { interview }\end{array}$ & $\begin{array}{l}\text { Role of the health } \\
\text { care worker }\end{array}$ & Gender & Date & Location & $\begin{array}{l}\text { Duration } \\
\text { (hours: } \\
\text { minutes: } \\
\text { seconds) }\end{array}$ \\
\hline 1 & $\begin{array}{l}\text { Family medicine } \\
\text { physician }\end{array}$ & Male & $16 / 07 / 2016$ & $\begin{array}{l}\text { The Comprehensive } \\
\text { Health Care Centre }\end{array}$ & $00: 34: 24$ \\
\hline 2 & Nurse & Male & $17 / 07 / 2016$ & $\begin{array}{l}\text { The Comprehensive } \\
\text { Health Care Centre. }\end{array}$ & $00: 30: 46$ \\
\hline 3 & Dietitian & Female & $24 / 07 / 2016$ & $\begin{array}{l}\text { The Comprehensive } \\
\text { Health Care Centre. }\end{array}$ & $00: 29: 11$ \\
\hline 4 & Nurse & Female & $01 / 08 / 2016$ & $\begin{array}{l}\text { The Comprehensive } \\
\text { Health Care Centre. }\end{array}$ & $00: 32: 59$ \\
\hline 5 & Lab technician & Female & $18 / 08 / 2016$ & $\begin{array}{l}\text { The Comprehensive } \\
\text { Health Care Centre. }\end{array}$ & $00: 28: 21$ \\
\hline 6 & Pharmacist & Female & $25 / 08 / 2016$ & $\begin{array}{l}\text { The Comprehensive } \\
\text { Health Care Centre. }\end{array}$ & $00: 20: 34$ \\
\hline 7 & Diabetic nurse & Female & $01 / 09 / 2016$ & $\begin{array}{l}\text { The Comprehensive } \\
\text { Health Care Centre. }\end{array}$ & $00: 23: 12$ \\
\hline 8 & Dentist & Female & $18 / 09 / 2016$ & $\begin{array}{l}\text { The Comprehensive } \\
\text { Health Care Centre. }\end{array}$ & $00: 30: 35$ \\
\hline 9 & Receptionist & Male & $21 / 09 / 2016$ & $\begin{array}{l}\text { The Comprehensive } \\
\text { Health Care Centre. }\end{array}$ & $00: 30: 48$ \\
\hline 10 & $\begin{array}{l}\text { Coordinator of } \\
\text { health promotion } \\
\text { programmes in } \\
\text { Directorate of } \\
\text { Health, The } \\
\text { Ministry of Heath }\end{array}$ & Female & $03 / 10 / 2016$ & $\begin{array}{l}\text { The Ministry of } \\
\text { Health }\end{array}$ & $00: 25: 26$ \\
\hline
\end{tabular}


Appendix 18. General Information of Family Carers who were interviewed in this study (n=6)

\begin{tabular}{|l|l|l|l|l|l|}
\hline $\begin{array}{l}\text { A number } \\
\text { of the } \\
\text { interview }\end{array}$ & $\begin{array}{l}\text { Relationship of } \\
\text { the family career } \\
\text { to the patient }\end{array}$ & Gender & Date & Location & $\begin{array}{l}\text { Duration } \\
\text { (hours: minutes: } \\
\text { seconds) }\end{array}$ \\
\hline 1 & Son & Male & $18 / 07 / 2016$ & $\begin{array}{l}\text { The Comprehensive } \\
\text { Health Care Centre }\end{array}$ & $00: 31: 51$ \\
\hline 2 & Son & Male & $09 / 08 / 2016$ & $\begin{array}{l}\text { The Comprehensive } \\
\text { Health Care Centre. }\end{array}$ & $00: 23: 09$ \\
\hline 3 & Spouse & Female & $11 / 08 / 2016$ & $\begin{array}{l}\text { The Comprehensive } \\
\text { Health Care Centre. }\end{array}$ & $00: 46: 31$ \\
\hline 4 & Spouse & Female & $25 / 08 / 2016$ & $\begin{array}{l}\text { The Comprehensive } \\
\text { Health Care Centre. }\end{array}$ & $00: 23: 19$ \\
\hline 5 & Spouse & Female & $06 / 10 / 2016$ & $\begin{array}{l}\text { The Comprehensive } \\
\text { Health Care Centre. }\end{array}$ & $00: 19: 42$ \\
\hline 6 & Husband & Male & $12 / 11 / 2016$ & $\begin{array}{l}\text { The Comprehensive } \\
\text { Health Care Centre. }\end{array}$ & $00: 20: 33$ \\
\hline
\end{tabular}


Appendix 19. All of the keywords used in the search process

\begin{tabular}{|c|c|c|c|}
\hline Type-2 diabetes & $\begin{array}{l}\text { Diabetes } \\
\text { complications }\end{array}$ & Diet & Knowledge \\
\hline Diabetes mellitus & $\begin{array}{l}\text { People with } \\
\text { diabetes/Type-2 } \\
\text { diabetes }\end{array}$ & Physical exercise/activity & $\begin{array}{l}\text { Perception of } \\
\text { Type-2 diabetes }\end{array}$ \\
\hline $\begin{array}{l}\text { Chronic diseases or } \\
\text { conditions }\end{array}$ & Arabs & $\begin{array}{l}\text { Self-monitoring blood } \\
\text { glucose (SMBG) }\end{array}$ & $\begin{array}{l}\text { Attitudes towards } \\
\text { diabetes }\end{array}$ \\
\hline Hyperglycaemia & Middle East countries & Diabetic foot care & Health beliefs \\
\hline $\begin{array}{l}\text { Poorly controlled } \\
\text { diabetes/Type- } 2 \\
\text { diabetes }\end{array}$ & Primary care centres & Adherence & Cultural norms \\
\hline Metabolic syndrome & Diabetic patients & Medication & $\begin{array}{l}\text { Social } \\
\text { habits/norms }\end{array}$ \\
\hline Obesity & Family & Eating habits & Awareness \\
\hline $\begin{array}{l}\text { Diabetic foot } \\
\text { syndrome }\end{array}$ & $\begin{array}{l}\text { Health care } \\
\text { workers/professionals }\end{array}$ & Religion & generosity \\
\hline Herbs & Jordan & WHO & Islam \\
\hline $\mathrm{T} 2 \mathrm{D}$ & Jordanians & IDF & Stress \\
\hline Retinopathy & UAE & Qatar & Turkey \\
\hline Sexual health & Saudi & Oman & Iran \\
\hline Prevalence & lifestyle & Adherence & Body mass index \\
\hline Overweight & Nutrition & Erectile dysfunction & $\begin{array}{l}\text { Famous Jordanian } \\
\text { cuisines }\end{array}$ \\
\hline Risk factors & periodontal disease & diabetic neuropathy & Honey \\
\hline dyslipidaemia & Metformin & Insulin & misconception \\
\hline Barriers & $\begin{array}{l}\text { Complementary and } \\
\text { alternative medicine }\end{array}$ & Malaysia & Kuwait \\
\hline Oral care & herbalists & Islamic Medicine & Arab medicine \\
\hline
\end{tabular}




\section{Appendix 20. Example of a Thematic Development}

Small segments from two focus group discussions that helped develop the key theme of 'Influence of the Jordanian socio-cultural context on patients' diabetes management plan'

\section{First phase of analysis}

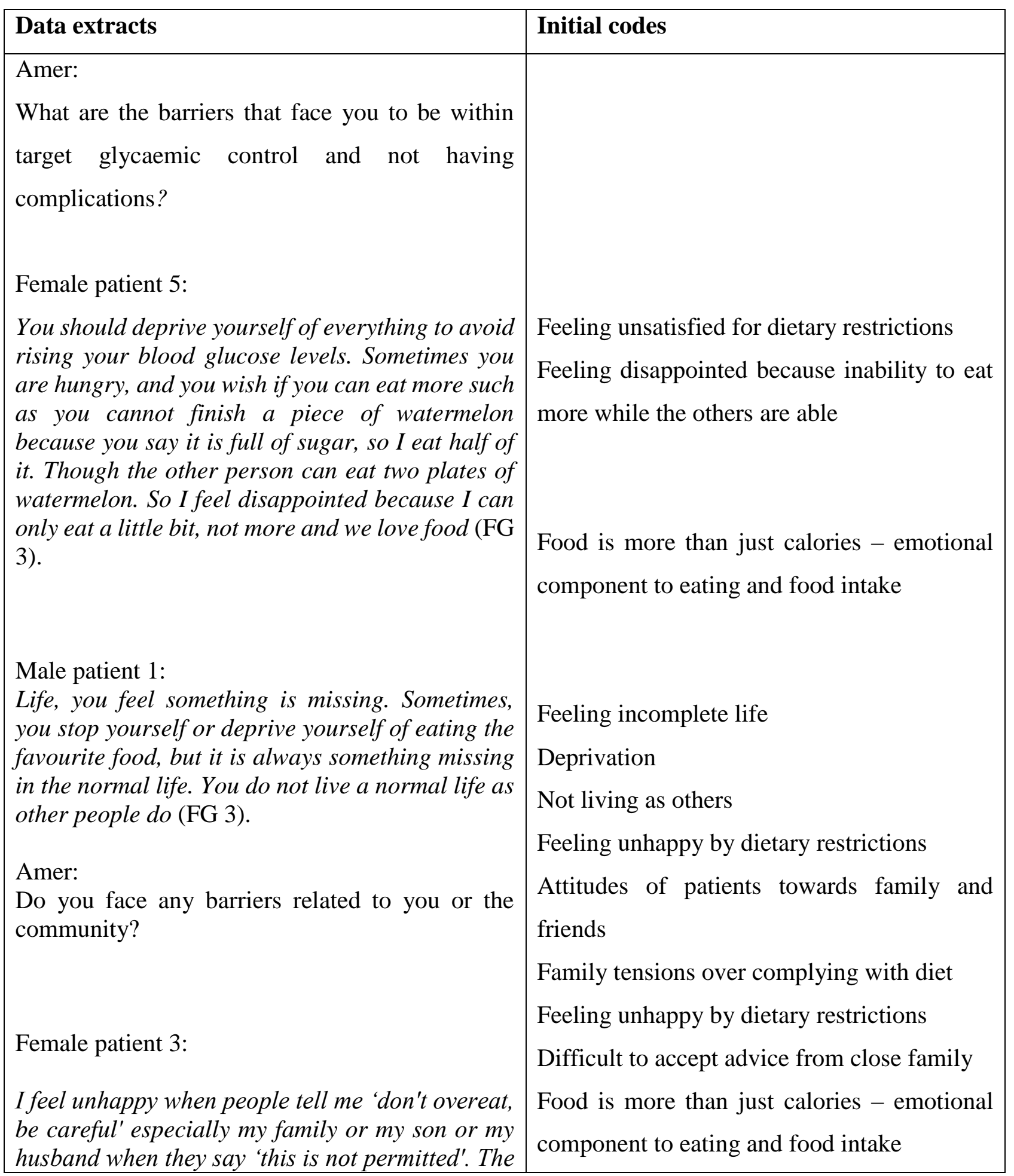


aim of that is decreasing the amount of eating, but the patient feels that his family don't want him to eat. When a person tells you don't eat something or don't eat grapes, you start eating it as intransigence. In some days, your body needs sugar, so you eat, and I may stay away from sweets, so I take sugar from fruits then a person comes and criticises you because it is full of sugar (FG 4).

Second phase of analysis

\begin{tabular}{|l|l|}
\hline Initial codes & Categories \\
\hline $\begin{array}{l}\text { Food is more than just calories - emotional component } \\
\text { to eating and food intake }\end{array}$ & Emotional component of food \\
Food is sensitive issue for the patient & \\
Family tensions over complying with diet & \\
Feeling unhappy by dietary restrictions & \\
Difficult to accept advice from close family & \\
Feeling disappointed because inability to eat more while \\
the others are able
\end{tabular}


Feeling of discrimination

The patient is unhappy with forbidden advice

Feeling hate at being deprived of food or leaving food in the plate

Patients are only able to eat limited amount of their favourite food such as sweets

\section{Third phase of analysis}

\begin{tabular}{|l|l|}
\hline Categories & Subtheme \\
\hline $\begin{array}{l}\text { Emotional component of food 'forbidden } \\
\text { phenomenon.' }\end{array}$ & Social component of food \\
$\begin{array}{l}\text { The role of food in the Jordanian socio- } \\
\text { cultural context }\end{array}$ & \\
\hline
\end{tabular}

\section{Fourth phase of analysis}

\begin{tabular}{|l|l|}
\hline Subthemes & The key theme \\
\hline Social component of food & $\begin{array}{l}\text { Influence of the Jordanian socio-cultural } \\
\text { context on patients' diabetes management } \\
\text { plan' }\end{array}$ \\
$\begin{array}{l}\text { Social factors influencing physical activity } \\
\text { and adherence to the medication regimen }\end{array}$ & \\
complementary therapies & \\
\hline
\end{tabular}


Appendix 21. Description of studies included in the literature review: (Authors, sampling strategy, research design, sample size, location and population)

\begin{tabular}{|c|c|c|c|c|c|c|}
\hline Authors & $\begin{array}{l}\text { Sampling } \\
\text { strategy }\end{array}$ & $\begin{array}{l}\text { Research } \\
\text { design }\end{array}$ & $\begin{array}{l}\text { Sample } \\
\text { size }\end{array}$ & Location & $\begin{array}{l}\text { Populatio } \\
\text { n }\end{array}$ & $\begin{array}{l}\text { Self-report } \\
\text { data }\end{array}$ \\
\hline $\begin{array}{l}\text { 1-(Yasein \& } \\
\text { Masa'd, 2011) }\end{array}$ & $\begin{array}{l}\text { Random } \\
\text { selection }\end{array}$ & Cross-sectional & 730 & $\begin{array}{l}\text { Jordan } \\
\text { University } \\
\text { Hospital }\end{array}$ & $\begin{array}{l}\text { Patients } \\
\text { with } \\
\text { chronic } \\
\text { conditions }\end{array}$ & No \\
\hline $\begin{array}{l}\text { 2- (Al-Amer et al., } \\
\text { 2011) }\end{array}$ & $\begin{array}{l}\text { Systematic } \\
\text { random } \\
\text { sampling }\end{array}$ & Cross-sectional & 649 & $\begin{array}{l}\text { Jordan } \\
\text { University } \\
\text { Hospital }\end{array}$ & $\begin{array}{l}\text { Patients } \\
\text { with } \\
\text { diabetes }\end{array}$ & Yes \\
\hline $\begin{array}{l}\text { 3- (Albikawi \& } \\
\text { Abuadas, 2015) }\end{array}$ & Convenience & Cross-sectional & 49 & $\begin{array}{l}\text { Diabetes } \\
\text { Specialized } \\
\text { Centre in } \\
\text { Amman- } \\
\text { Jordan }\end{array}$ & $\begin{array}{l}\text { Patients } \\
\text { with Type- } \\
2 \text { diabetes }\end{array}$ & Yes \\
\hline $\begin{array}{l}\text { 4-(Al-Maskari et } \\
\text { al., 2013) }\end{array}$ & $\begin{array}{l}\text { Random } \\
\text { selection }\end{array}$ & Cross-sectional & 575 & $\begin{array}{l}\text { United Arab } \\
\text { Emiratis }\end{array}$ & $\begin{array}{l}\text { Patients } \\
\text { with } \\
\text { diabetes }\end{array}$ & Yes \\
\hline $\begin{array}{l}\text { 5-(Al Omari et al., } \\
\text { 2009) }\end{array}$ & Convenience & Cross-sectional & 337 & $\begin{array}{l}\text { A primary } \\
\text { care centre }\end{array}$ & $\begin{array}{l}\text { Type-2 } \\
\text { diabetic } \\
\text { patients }\end{array}$ & No \\
\hline $\begin{array}{l}\text { 6-(Al-Sarihin et } \\
\text { al., 2012) }\end{array}$ & Convenience & Cross-sectional & 100 & $\begin{array}{l}\text { King } \\
\text { Hussein } \\
\text { Hospital }\end{array}$ & $\begin{array}{l}\text { Patients } \\
\text { with } \\
\text { diabetes }\end{array}$ & No \\
\hline $\begin{array}{ll}\text { 7-(Al-Keilani et } \\
\text { al., 2017) }\end{array}$ & Convenience & Cross-sectional & 1079 & $\begin{array}{l}18 \text { hospitals } \\
\text { and health } \\
\text { care centres } \\
\text { in Jordan }\end{array}$ & $\begin{array}{l}\text { Patients } \\
\text { with } \\
\text { diabetes }\end{array}$ & Yes \\
\hline $\begin{array}{l}\text { 8-(Khawaja et al., } \\
\text { 2018). }\end{array}$ & Convenience & Cross-sectional & 1003 & $\begin{array}{l}\text { The } \\
\text { National }\end{array}$ & $\begin{array}{l}\text { Patients } \\
\text { with Type- } \\
2 \text { diabetes }\end{array}$ & No \\
\hline
\end{tabular}




\begin{tabular}{|c|c|c|c|c|c|c|}
\hline & & & & $\begin{array}{l}\text { Diabetes } \\
\text { Centre }\end{array}$ & & \\
\hline $\begin{array}{l}\text { 9-(Khatib et al., } \\
\text { 2007) }\end{array}$ & Convenience & Cross-sectional & 150 & Israel & $\begin{array}{l}\text { Patients } \\
\text { with } \\
\text { diabetes }\end{array}$ & Yes \\
\hline $\begin{array}{l}\text { 10-(Salayta \& } \\
\text { Aleyadeh, 2010). }\end{array}$ & Convenience & $\begin{array}{l}\text { Longitudinal } \\
\text { cohort, } \\
\text { prospective } \\
\text { study }\end{array}$ & 60 & $\begin{array}{l}\text { King } \\
\text { Hussein } \\
\text { Medical } \\
\text { Centre }\end{array}$ & $\begin{array}{l}\text { Patients } \\
\text { with } \\
\text { diabetes }\end{array}$ & No \\
\hline $\begin{array}{l}\text { 11-(Abu-Qamar } \\
\& \text { Wilson, 2011). }\end{array}$ & $\begin{array}{l}\text { Purposeful } \\
\text { sampling }\end{array}$ & Qualitative & 7 & $\begin{array}{l}\text { Six } \\
\text { hospitals } \\
\text { throughout } \\
\text { Jordan }\end{array}$ & $\begin{array}{l}\text { Patients } \\
\text { with } \\
\text { diabetes }\end{array}$ & No \\
\hline $\begin{array}{l}\text { 12-(Al-Kaabi et } \\
\text { al., 2015) }\end{array}$ & $\begin{array}{l}\text { Random } \\
\text { selection }\end{array}$ & Cross-sectional & 422 & UAE & $\begin{array}{l}\text { Patients } \\
\text { with } \\
\text { diabetes }\end{array}$ & Yes \\
\hline $\begin{array}{l}\text { 13-(Odhayani, et } \\
1 ., 2015)\end{array}$ & Convenience & Cross-sectional & 350 & $\begin{array}{l}\text { Saudi } \\
\text { Arabia }\end{array}$ & $\begin{array}{l}\text { Patients } \\
\text { with } \\
\text { diabetes }\end{array}$ & Yes \\
\hline $\begin{array}{l}\text { 14-(Wazaify et al., } \\
\text { 2013) }\end{array}$ & $\begin{array}{l}\text { Random } \\
\text { selection }\end{array}$ & Cross-sectional & 700 & $\begin{array}{l}\text { Jordan } \\
\text { University } \\
\text { Hospital }\end{array}$ & $\begin{array}{l}\text { Patients } \\
\text { with } \\
\text { chronic } \\
\text { conditions }\end{array}$ & Yes \\
\hline $\begin{array}{l}\text { 15-(Skovlund \& } \\
\text { Peyrot, 2005). }\end{array}$ & Convenience & Cross-sectional & 5000 & $\begin{array}{l}13 \text { different } \\
\text { countries in } \\
\text { Europe, } \\
\text { Asia, } \\
\text { Australia } \\
\text { and USA }\end{array}$ & $\begin{array}{l}\text { Patients } \\
\text { with } \\
\text { diabetes }\end{array}$ & No \\
\hline $\begin{array}{l}\text { 16-(Al- } \\
\text { Khawaldeh et al., } \\
\text { 2012) }\end{array}$ & Convenience & $\begin{array}{l}\text { Cross-sectional } \\
\text { survey }\end{array}$ & 223 & $\begin{array}{l}\text { The } \\
\text { National } \\
\text { Diabetes } \\
\text { Centre }\end{array}$ & $\begin{array}{l}\text { Patients } \\
\text { with Type- } \\
2 \text { diabetes }\end{array}$ & Yes \\
\hline
\end{tabular}




\begin{tabular}{|c|c|c|c|c|c|c|}
\hline $\begin{array}{l}\text { 17-(Arifulla et } \\
\text { al.,2014) }\end{array}$ & $\begin{array}{l}\text { Purposeful } \\
\text { sampling }\end{array}$ & $\begin{array}{l}\text { Cross-sectional } \\
\text { survey }\end{array}$ & 132 & UAE & $\begin{array}{l}\text { Patients } \\
\text { with Type- } \\
2\end{array}$ & Yes \\
\hline $\begin{array}{l}\text { 18- (Awwad et al., } \\
\text { 2015) }\end{array}$ & Convenience & $\begin{array}{l}\text { Cross-sectional } \\
\text { survey }\end{array}$ & 902 & $\begin{array}{l}\text { The } \\
\text { University } \\
\text { of Jordan } \\
\text { Hospital } \\
\text { and Jordan } \\
\text { Hospital }\end{array}$ & $\begin{array}{l}\text { Patients } \\
\text { with } \\
\text { chronic } \\
\text { conditions }\end{array}$ & Yes \\
\hline $\begin{array}{l}\text { 19-(Basheti et al., } \\
\text { 2016) }\end{array}$ & $\begin{array}{l}\text { Random } \\
\text { selection. }\end{array}$ & $\begin{array}{l}\text { Cross-sectional } \\
\text { survey }\end{array}$ & 167 & Amman & $\begin{array}{l}\text { Patients } \\
\text { with } \\
\text { chronic } \\
\text { conditions }\end{array}$ & Yes \\
\hline $\begin{array}{l}\text { 20- (Ozcelik et al., } \\
\text { 2010) }\end{array}$ & $\begin{array}{l}\text { Purposeful } \\
\text { sampling }\end{array}$ & $\begin{array}{l}\text { Cross-sectional } \\
\text { survey }\end{array}$ & 164 & Turkey & $\begin{array}{l}\text { Patients } \\
\text { with Type- } \\
2 \text { diabetes }\end{array}$ & Yes \\
\hline $\begin{array}{l}\text { 21-(Bakkar et al., } \\
\text { 2017) }\end{array}$ & $\begin{array}{l}\text { Random } \\
\text { selection }\end{array}$ & $\begin{array}{l}\text { Cross-sectional } \\
\text { survey }\end{array}$ & 237 & $\begin{array}{l}\text { General } \\
\text { population; } \\
\text { Amman, } \\
\text { Zarqa } \\
\text { and Irbid }\end{array}$ & $\begin{array}{l}\text { Patients } \\
\text { with Type- } \\
2 \text { diabetes }\end{array}$ & No \\
\hline $\begin{array}{l}\text { 22- (Al-Adsani et } \\
\text { al., 2009) }\end{array}$ & Convenience & $\begin{array}{l}\text { Cross-sectional } \\
\text { survey }\end{array}$ & 5114 & Kuwait & $\begin{array}{l}\text { Patients } \\
\text { with Type- } \\
2 \text { diabetes }\end{array}$ & Yes \\
\hline $\begin{array}{l}\text { 23- (El-Qudah, } \\
2016)\end{array}$ & Convenience & $\begin{array}{l}\text { Cross-sectional } \\
\text { survey }\end{array}$ & 180 & $\begin{array}{l}\text { Different } \\
\text { hospitals in } \\
\text { Amman, } \\
\text { Jordan. }\end{array}$ & $\begin{array}{l}\text { Jordanian } \\
\text { females } \\
\text { with } \\
\text { diabetes }\end{array}$ & Yes \\
\hline $\begin{array}{l}\text { 24-(Wazaify et al., } \\
\text { 2011) }\end{array}$ & $\begin{array}{l}\text { Random } \\
\text { selection }\end{array}$ & Cross-sectional & 1000 & $\begin{array}{l}\text { The } \\
\text { National } \\
\text { Diabetes } \\
\text { Centre }\end{array}$ & $\begin{array}{l}\text { Patients } \\
\text { with } \\
\text { diabetes }\end{array}$ & Yes \\
\hline
\end{tabular}




\begin{tabular}{|c|c|c|c|c|c|c|}
\hline $\begin{array}{l}\text { 25-(Alkhazrajy et } \\
\text { al., 2014) }\end{array}$ & $\begin{array}{l}\text { Convenience } \\
\text { sample }\end{array}$ & $\begin{array}{l}\text { Cross-sectional } \\
\text { survey }\end{array}$ & 400 & $\begin{array}{l}10 \text { primary } \\
\text { care centres } \\
\text { in Iraq }\end{array}$ & $\begin{array}{l}\text { Health } \\
\text { care } \\
\text { workers }\end{array}$ & Yes \\
\hline $\begin{array}{l}\text { 26- (Darawad et } \\
\text { al.,2016) }\end{array}$ & convenience & $\begin{array}{l}\text { Cross-sectional } \\
\text { survey }\end{array}$ & 115 & $\begin{array}{l}\text { Four } \\
\text { hospitals in } \\
\text { Jordan }\end{array}$ & $\begin{array}{l}\text { Patients } \\
\text { with } \\
\text { diabetes }\end{array}$ & Yes \\
\hline $\begin{array}{l}\text { 27-(Sulaiman et } \\
\text { al., 2018) }\end{array}$ & $\begin{array}{l}\text { Random } \\
\text { selection }\end{array}$ & $\begin{array}{l}\text { Cross-sectional } \\
\text { survey }\end{array}$ & 872 & UAE & $\begin{array}{l}\text { General } \\
\text { population }\end{array}$ & No \\
\hline $\begin{array}{l}\text { 28-(Al-Kaabi et } \\
\text { al., 2009) }\end{array}$ & $\begin{array}{l}\text { Random } \\
\text { selection. }\end{array}$ & $\begin{array}{l}\text { Cross-sectional } \\
\text { survey }\end{array}$ & 390 & UAE & $\begin{array}{l}\text { Patients } \\
\text { with Type- } \\
2 \text { diabetes }\end{array}$ & Yes \\
\hline $\begin{array}{l}\text { 29-(Bawadi \& } \\
\text { Abu-Jamous, } \\
\text { 2013). }\end{array}$ & Convenience & $\begin{array}{l}\text { Cross-sectional } \\
\text { survey }\end{array}$ & 750 & $\begin{array}{l}\text { King } \\
\text { Abdulla } \\
\text { University } \\
\text { Hospital }\end{array}$ & $\begin{array}{l}\text { Patients } \\
\text { with Type- } \\
2 \text { diabetes }\end{array}$ & Yes \\
\hline $\begin{array}{l}\text { 30- (Abdel-Aal et } \\
\text { al.,2008) }\end{array}$ & Convenience & $\begin{array}{l}\text { Cross-sectional } \\
\text { survey }\end{array}$ & 702 & $\begin{array}{l}\text { The } \\
\text { National } \\
\text { Diabetes } \\
\text { Centre }\end{array}$ & $\begin{array}{l}\text { Patients } \\
\text { with Type- } \\
2 \text { diabetes }\end{array}$ & No \\
\hline $\begin{array}{l}\text { 31- (Khatib et al., } \\
\text { 2006) }\end{array}$ & Convenience & $\begin{array}{l}\text { Cross-sectional } \\
\text { survey }\end{array}$ & 988 & $\begin{array}{l}\text { The } \\
\text { National } \\
\text { Diabetes } \\
\text { Centre }\end{array}$ & $\begin{array}{l}\text { Patients } \\
\text { with } \\
\text { diabetes }\end{array}$ & No \\
\hline $\begin{array}{l}\text { 32-(Al-Saeedi et } \\
\text { al., 2002) }\end{array}$ & $\begin{array}{l}\text { Random } \\
\text { selection }\end{array}$ & $\begin{array}{l}\text { Cross-sectional } \\
\text { survey }\end{array}$ & 1039 & $\begin{array}{l}\text { Different } \\
\text { primary } \\
\text { health care } \\
\text { centres in } \\
\text { Saudi } \\
\text { Arabia }\end{array}$ & $\begin{array}{l}\text { Patients } \\
\text { with } \\
\text { diabetes }\end{array}$ & Yes \\
\hline $\begin{array}{l}\text { 33- (Al-Bdour et } \\
\text { al., 2008) }\end{array}$ & Convenience & $\begin{array}{l}\text { Cross-sectional } \\
\text { survey }\end{array}$ & 1961 & $\begin{array}{l}\text { Jordan } \\
\text { University } \\
\text { Hospital }\end{array}$ & $\begin{array}{l}\text { Patients } \\
\text { with } \\
\text { diabetes }\end{array}$ & No \\
\hline
\end{tabular}




\begin{tabular}{|c|c|c|c|c|c|c|}
\hline $\begin{array}{l}34-\text { (Ajlouni et } \\
\text { al.,2008) }\end{array}$ & $\begin{array}{l}\text { Random } \\
\text { selection }\end{array}$ & $\begin{array}{l}\text { Cross-sectional } \\
\text { survey }\end{array}$ & 1121 & $\begin{array}{l}\text { A } \\
\text { comprehens } \\
\text { ive health } \\
\text { centre }\end{array}$ & $\begin{array}{l}\text { General } \\
\text { population }\end{array}$ & No \\
\hline $\begin{array}{l}\text { 35- (Al-Amer et } \\
\text { al., 2008) }\end{array}$ & $\begin{array}{l}\text { Random } \\
\text { selection }\end{array}$ & Cross-sectional & 1000 & $\begin{array}{l}\text { The national } \\
\text { diabetes } \\
\text { centre }\end{array}$ & $\begin{array}{l}\text { Patients } \\
\text { with Type- } \\
2 \text { diabetes }\end{array}$ & No \\
\hline $\begin{array}{l}\text { 36- (Al-Qazaz et } \\
\text { al., 2011) }\end{array}$ & Convenience & Qualitative & 12 & Malaysia & $\begin{array}{l}\text { Patients } \\
\text { with Type- } \\
2 \text { diabetes }\end{array}$ & No \\
\hline $\begin{array}{l}\text { 37- (Ali et al., } \\
\text { 2008) }\end{array}$ & $\begin{array}{l}\text { Random } \\
\text { selection }\end{array}$ & Cross-sectional & 1137 & $\begin{array}{l}\text { The } \\
\text { National } \\
\text { Diabetes } \\
\text { Centre }\end{array}$ & $\begin{array}{l}\text { Female } \\
\text { patients } \\
\text { with } \\
\text { diabetes, } \\
\text { family } \\
\text { carers and } \\
\text { HCWs }\end{array}$ & Yes \\
\hline $\begin{array}{l}\text { 38- (Rabiu et al., } \\
\text { 2015) }\end{array}$ & $\begin{array}{l}\text { Random } \\
\text { selection }\end{array}$ & Cross-sectional & 3780 & $\begin{array}{l}\text { North } \\
\text { Jordan }\end{array}$ & $\begin{array}{l}\text { General } \\
\text { population }\end{array}$ & No \\
\hline $\begin{array}{l}\text { 39- (Jammal et al., } \\
\text { 2013) }\end{array}$ & $\begin{array}{l}\text { Random } \\
\text { selection }\end{array}$ & Cross-sectional & 127 & $\begin{array}{l}\text { The } \\
\text { National } \\
\text { Diabetes } \\
\text { Centre and } \\
\text { diabetes } \\
\text { clinic }\end{array}$ & $\begin{array}{l}\text { Patients } \\
\text { with Type- } \\
2 \text { diabetes }\end{array}$ & No \\
\hline $\begin{array}{l}\text { 40- (Khattab et al., } \\
\text { 2010) }\end{array}$ & $\begin{array}{l}\text { Systematic } \\
\text { random sample }\end{array}$ & Cross-sectional & 917 & $\begin{array}{l}\text { The } \\
\text { National } \\
\text { Diabetes } \\
\text { Centre }\end{array}$ & $\begin{array}{l}\text { Patients } \\
\text { with Type- } \\
2 \text { diabetes }\end{array}$ & Yes \\
\hline $\begin{array}{l}\text { 41- (Al Jamal \& } \\
\text { Ibrahim, 2011) }\end{array}$ & $\begin{array}{l}\text { Random } \\
\text { selection }\end{array}$ & $\begin{array}{l}\text { Pre/post } \\
\text { randomised } \\
\text { trial }\end{array}$ & 45 & $\begin{array}{l}\text { Al Mafraq } \\
\text { Government } \\
\text { al Hospital } \\
\text { in Jordan }\end{array}$ & $\begin{array}{l}\text { Patients } \\
\text { with Type- } \\
2 \text { diabetes }\end{array}$ & No \\
\hline
\end{tabular}




\begin{tabular}{|l|l|l|l|l|l|l|}
\hline $\begin{array}{l}\text { 42-(Bakhotmah \& } \\
\text { Alzahrani, 2010) }\end{array}$ & Convenience & Cross-sectional & 1634 & Saudi & $\begin{array}{l}\text { Patients } \\
\text { with } \\
\text { diabetes }\end{array}$ & Yes \\
\hline $\begin{array}{l}\text { 43- (Barghouti et } \\
\text { al., 2015) }\end{array}$ & $\begin{array}{l}\text { Random } \\
\text { selection }\end{array}$ & Cross-sectional & 3196 & $\begin{array}{l}\text { Three } \\
\text { regions of } \\
\text { Jordan }\end{array}$ & $\begin{array}{l}\text { General } \\
\text { population }\end{array}$ & Yes \\
\hline $\begin{array}{l}\text { 44- (Bakri et al., } \\
\text { 2012) }\end{array}$ & $\begin{array}{l}\text { Random } \\
\text { selection }\end{array}$ & $\begin{array}{l}\text { Cross-sectional } \\
\text { survey }\end{array}$ & 1000 & $\begin{array}{l}\text { The } \\
\text { National } \\
\text { Diabetes } \\
\text { Centre }\end{array}$ & $\begin{array}{l}\text { Patients } \\
\text { with } \\
\text { diabetes }\end{array}$ & No \\
\hline $\begin{array}{l}\text { 45- (Sabbah \& } \\
\text { Alshehri, 2014). }\end{array}$ & $\begin{array}{l}\text { Random } \\
\text { selection }\end{array}$ & $\begin{array}{l}\text { Cross-sectional } \\
\text { study }\end{array}$ & 386 & $\begin{array}{l}\text { Saudi } \\
\text { Arabia }\end{array}$ & $\begin{array}{l}\text { Patients } \\
\text { with } \\
\text { diabetes }\end{array}$ & Yes \\
\hline $\begin{array}{l}\text { 46-(Gopalan et } \\
\text { al., 2014). }\end{array}$ & Convenience & $\begin{array}{l}\text { Cross-sectional } \\
\text { study }\end{array}$ & 177 & USA & $\begin{array}{l}\text { Patients } \\
\text { with } \\
\text { diabetes }\end{array}$ & No \\
\hline $\begin{array}{l}\text { 47-(Jarab } \\
\text { al.,2018) }\end{array}$ & $\begin{array}{l}\text { Purposive } \\
\text { sampling }\end{array}$ & Qualitative & 36 & $\begin{array}{l}\text { The Royal } \\
\text { Medical } \\
\text { Services } \\
\text { Hospital }\end{array}$ & $\begin{array}{l}\text { Patients } \\
\text { with Type- } \\
2 \text { diabetes }\end{array}$ & No \\
\hline
\end{tabular}




\section{Appendix 22. Publications and conference presentations from this study}

\section{Papers published}

-Al-Sahouri, A., Merrell, J. and Snelgrove, S. (2019) Attitudes, Knowledge, and Perceptions of Patients Regarding Type-2 Diabetes in Jordan. Journal of Diabetes Mellitus, 9, 1-13. https://doi.org/10.4236/jdm.2019.91001.

2- Al-Sahouri, A., Merrell, J. and Snelgrove, S. (2019). Barriers to good glycaemic control levels and adherence to diabetes management plan in adults with Type-2 diabetes in Jordan: A literature review. Patient Preference and Adherence. In press.

\section{$\underline{\text { Oral presentations }}$}

-An Ethnographic Study of The Factors Associated with Poor Glycaemic Control Levels Among People with Type 2 Diabetes Accessing a Primary Care Centre in Jordan. A European videoconference to present and discuss PhD research 2016, Swansea University, UK.

-An Ethnographic Study of The Factors Associated with Poor Glycaemic Control Levels Among People with Type 2 Diabetes Accessing a Primary Care Centre in Jordan. 3 Minute Thesis Competition 2017, Swansea University, UK.

-An Ethnographic Study of The Factors Associated with Poor Glycaemic Control Levels Among People with Type 2 Diabetes Accessing a Primary Care Centre in Jordan. College of Human and Health Sciences Annual Research Conference 2017, Swansea University, UK.

-An Ethnographic Study of The Factors Associated with Poor Glycaemic Control Levels Among People with Type 2 Diabetes Accessing a Primary Care Centre in Jordan. A European videoconference to present and discuss PhD research 2017, Swansea University, UK.

-Herbal Usage among People with Type 2 Diabetes Accessing a Primary Care Centre in Jordan. College of Human and Health Sciences - Promoting Health and Wellbeing conference 2018. Swansea University, United Kingdom.

\section{$\underline{\text { Poster presentations }}$}

Herbal Usage among People with Type 2 Diabetes Accessing a Primary Care Centre in Jordan. International Symposium 'Transforming Community and Public Health: Inspiring Future Generations' 2018. Swansea University, United Kingdom. 


\section{References}

Abdel-Aal, N., Ahmad, A., Froelicher, E., Batieha, M., Hamza, M., \& Ajlouni, K. (2008). Prevalence of dyslipidaemia in patients with type 2 diabetes in Jordan. Saudi medical journal, 29(10), 1423-1428.

Abolghasemi, R., \& Sedaghat, M. (2014). The Patient's Attitude towards Type 2 Diabetes Mellitus, a Qualitative Study. Journal of Religion and Health, 54(4), 1191-1205.doi: 10.1007/s10943-014-9848-9.

Abu-Qamar, M., \& Wilson, A. (2011). Foot care within the Jordanian health care system: A qualitative inquiry of patient's perspectives. Australian Journal of Advanced Nursing, 29(1), 28-36.

Abu-Sobah, M. (2013, October 9). Physical education: the less fortunate despite the students thirst for it. Alghad News. Retrieved from https://www.alghad.com/articles.

Adwan, M., \& Najjar, Y. (2013). The Relationship between Demographic Variables and Diabetes Self-Management in Diabetic Patients in Amman City/Jordan. Global Journal of Health Science, 5(2). doi:10.5539/gjhs.v5n2p213.

Ahmed, H. (2016). Ethnopharmacobotanical study on the medicinal plants used by herbalists in Sulaymaniyah Province, Kurdistan, Iraq. Journal of Ethnobiology and Ethnomedicine, 12(1).doi:10.1186/s13002-016-0081-3.

Ajlouni, K., Jaddou, H., \& Batieha, A. (1998). Diabetes and impaired glucose tolerance in Jordan: Prevalence and associated risk factors. Journal of Internal Medicine, 244(4), 317-323. doi:10.1046/j.1365-2796.1998.00369.

Ajlouni, K., Khader, Y. S., Batieha, A., Ajlouni, H., \& El-Khateeb, M. (2008). An increase in prevalence of diabetes mellitus in Jordan over 10 years. Journal of Diabetes and Its Complications, 22(5), 317-324. doi:10.1016/j.jdiacomp.2007.01.004.

Ajzen, I. (1991). The theory of planned behaviour. Organizational behaviour and human decision processes, 50(2), 179-211.

Al-Adsani, A., Moussa, M., Al-Jasem, L., Abdella, N., \& Al-Hamad, N. (2009). The level and determinants of diabetes knowledge in Kuwaiti adults with type 2 diabetes. Diabetes and Metabolism, 35(2), 121-128. 
Al-Amer, R., Khader, Y., Malas, S., Abu-Yaghi, N., Al-Bdour, M., \& Ajlouni, K. (2008). Prevalence and risk factors of diabetic retinopathy among Jordanian patients with type 2 diabetes. Digital journal of ophthalmology: DJO, 14, 42.

Al-Amer, R., Sobeh, M., Zayed, A., \& Al-Domi, H. (2011). Depression among adults with diabetes in Jordan: Risk factors and relationship to blood sugar control. Journal of Diabetes and Its Complications, 25(4), 247-252. doi:10.1016/j.jdiacomp.2011.03.001.

Alam, F., Islam, M., Gan, S., \& Khalil, M. (2014). Honey: A Potential Therapeutic Agent for Managing Diabetic Wounds. Evidence-Based Complementary and Alternative Medicine, 2014, 1-16. doi:10.1155/2014/169130.

Albargawi, M., Snethen, J., Gannass, A., \& Kelber, S. (2016). Perception of persons with type 2 diabetes mellitus in Saudi Arabia. International Journal of Nursing Sciences, 3(1), 39-44. doi:10.1016/j.ijnss.2016.02.007.

Al-Bdour, M., Al-Till, M., \& Samra, K. (2008). Risk factors for diabetic retinopathy among Jordanian diabetics. Middle East African journal of ophthalmology, 15(2), 77.

Albikawi, Z., \& Abuadas, M. (2015). Diabetes self-care management behaviours among Jordanian type two diabetes patients. American International Journal of Contemporary Research, 5(3), 87-95.

Albinali, H. (2013). Arab or Islamic medicine? Heart views: the official journal of the Gulf Heart Association, 14(1), 41.

Alefan, Q., Tashman, K., Mukattash, T., \& Azzam, S. (2014). OHP-001 Medicines shortages in $\mathrm{MOH}$ hospitals in Jordan: Problems \& solutions. European Journal of Hospital Pharmacy, 21(Suppl 1). doi:10.1136/ejhpharm-2013-000436.453.

Alhabashneh, R., Khader, Y., Herra, Z., \& Asa'Ad, F. (2015). The association between periodontal disease and metabolic syndrome among outpatients with diabetes in Jordan. Journal of Diabetes \& Metabolic Disorders, 14(1).doi:10.1186/s40200-015-0207-5.

Al-Hariri, M. (2013). The Local Effects of Honey on Diabetic Foot Disorder. Pakistan Journal of Physiology, 9(1), 32-35.

Ali, R., Al Hajeri, R., Khader, Y., Shegem, N., \& Ajlouni, K. (2008). Sexual dysfunction in Jordanian diabetic women. Diabetes care 31:1580-1581.doi: 10.2337/dc08-0081. 
Aljadid, R. (2018, May 29). Amman ranked most expensive Arab city, 28th worldwide. The Jordan Times. Retrieved from http://jordantimes.com/news/local/amman-ranked-mostexpensive-arab-city-28th-worldwide.

Al Jamal, A., \& Ibrahim, A. (2011). Effects of olive oil on lipid profiles and blood glucose in type 2 diabetic patients. Int J Diabetes Metab, 19, 19-22.

Al-Kaabi, J., Al-Maskari, F., Saadi, H., Afandi, B., Parkar, H., \& Nagelkerke, N. (2009). Physical Activity and Reported Barriers to Activity Among Type 2 Diabetic Patients in the United Arab Emirates. The Review of Diabetic Studies, 6(4), 271-278. doi:10.1900/rds.2009.6.271.

Al-Kaabi, J., Maskari, F., Cragg, P., Afandi, B., \& Souid, A. (2015). Illiteracy and diabetic foot complications. Primary Care Diabetes, 9(6), 465-472. doi:10.1016/j.pcd.2015.04.008.

Al-Keilani, M., Almomani, B., Al-Sawalha, N., \& Shhabat, B. (2017). Self-monitoring of blood glucose among patients with diabetes in Jordan: Perception, adherence, and influential $\begin{array}{llll}\text { factors. Diabetes } \quad \text { Research } & \text { Clinical }\end{array}$ doi:10.1016/j.diabres.2017.01.005.

Al-Khawaldeh, O., Al-Hassan, M., \& Froelicher, E. (2012). Self-efficacy, self-management, and glycaemic control in adults with type 2 diabetes mellitus. Journal of Diabetes and Its Complications, 26(1), 10-16. doi:10.1016/j.jdiacomp.2011.11.002.

Alkhazrajy, L., Habib, H., \& Alsudani, Z. (2014). Perception of Iraqi primary health care providers about herbs in practice. European Journal of Botany, Plant Science and Phytology, $l 1(1), 27-52$.

Al-Maskari, F., El-Sadig, M., Al-Kaabi, J., Afandi, B., Nagelkerke, N., \& Yeatts, K. (2013). Knowledge, Attitude and Practices of Diabetic Patients in the United Arab Emirates. PLoS ONE, 8(1). doi:10.1371/journal.pone.0052857.

Al-Mutairi, R., Bawazir, A., Ahmed, A., \& Jradi, H. (2015). Health Beliefs Related to Diabetes Mellitus Prevention among Adolescents in Saudi Arabia. Sultan Qaboos University Medical Journal, 15(3). doi:10.18295/squmj.2015.15.03.015.

Al Omari, M., Khader, Y., Dauod, A., Al-Akour, N., Khassawneh, A., Al-Ashker, E., \& AlShdifat, A. (2009). Glycaemic control among patients with type 2 diabetes mellitus treated in 
primary care setting in Jordan. Primary Care Diabetes, 3(3), 173-179. doi:10.1016/j.pcd.2009.08.004.

Al-Nsour, A. (2017, January 2). Medical Exemption. Alrai Newspaper. Retrieved from http://alrai.com/article/1034501.

Al-Nsour, M., Zindah, M., Belbeisi, A., Hadaddin, R., Brown, D., \& Walke, H. (2012). Prevalence of Selected Chronic, No communicable Disease Risk Factors in Jordan: Results of the 2007 Jordan Behavioural Risk Factor Surveillance Survey. Preventing Chronic Disease. doi:10.5888/pcd9.110077.

Al-Rajby, D. (2017, February 14). Physical education in the Arab world is empty of its content. Alaraby News. Retrieved from https://www.alaraby.co.uk/specialpages.

Al-Qazaz, H., Hassali, M., Shafie, A., Sulaiman, S., \& Sundram, S. (2011). Perception and knowledge of patients with type 2 diabetes in Malaysia about their disease and medication: A qualitative study. Research in Social and Administrative Pharmacy, 7(2), 180-191. doi:10.1016/j.sapharm.2010.04.005.

Alrahbi, H. (2014). Diabetes self-management (DSM) in Omani with type-2

diabetes. International Journal of Nursing Sciences, 1(4), 352-359.

doi:10.1016/j.ijnss.2014.09.002.

Alrawi, S., \& Fetters, M. (2012). Traditional Arabic \& Islamic Medicine: A Conceptual Model for Clinicians and Researchers. Global Journal of Health Science, 4(3). doi:10.5539/gjhs.v4n3p164.

Alrawi, S., Khidir, A., Elnashar, M., Abdelrahim, H., Killawi, A., Hammoud, M., \& Fetters, M. (2017). Traditional Arabic \& Islamic medicine: Validation and empirical assessment of a conceptual model in Qatar. BMC Complementary and Alternative

Medicine, 17(1).doi:10.1186/s12906-017-1639.

Al-Saeedi, M., Elzubier, A., Al-Dawood, K., \& Bahnasi, A. (2002). Dietary misconceptions among diabetic patients in Makkah city, Saudi Arabia. Journal of family \& community medicine, 9(2), 41.

Alsairafi, Z., Taylor, K., Smith, F., \& Alattar, A. (2016). Patients' management of type 2 diabetes in Middle Eastern countries: Review of studies. Patient Preference and Adherence, 1051. doi:10.2147/ppa.s104335. 
Al-Sarihin, K., Bani-Khaled, M., Haddad, F., \& Althwabia, I. (2012). Diabetes knowledge among patients with diabetes mellitus at King Hussein Hospital. JRMS, 19(1), 72-77.

Al-Shudifat, A., Johannessen, A., Azab, M., Abumweis, S., Agraib, L., \& Tayyem, R. (2017). Risk factors for coronary artery disease in patients undergoing elective coronary angiography in Jordan. BMC Cardiovascular Disorders, 17(1).doi: 10.1186/s12872-017-0620-4.

Alsunni, A., Albaker, W., \& Badar0, A. (2014). Determinants of misconceptions about diabetes among Saudi diabetic patients attending diabetes clinic at a tertiary care hospital in Eastern Saudi Arabia. Journal of Family and Community Medicine, 21(2), 93. doi:10.4103/22308229.134764.

Alzahrani, H., Wang, D., Alzahrani, A., \& Hu, F. (2015). Incidence of diabetic foot disorders in patients with diabetes in Jeddah, Saudi Arabia. International Journal of Diabetes in Developing Countries, 35(2), 115-122.doi:10.1007/s13410-014-0272-1.

American Diabetes Association. (2013). Standards of medical care in diabetes. Diabetes care, 36 (Suppl 1), S11-S66.doi: 10.2337/dc13-S011.

American Diabetes Association. (2014). Diagnosis and classification of diabetes mellitus. Diabetes care, 37(Supplement 1), S81-S90.doi: 10.2337/dc14-S081.

American Diabetes Association. (2015a). Insulin Basics. Retrieved July 16, 2015, from http://www.diabetes.org/living-with-diabetes/treatment-and-care/medication/insulin/insulinbasics.html.

American Diabetes Association. (2015b). Fats. Retrieved August 13, 2015, from http://www.diabetes.org/food-and-fitness/food/what-can-i-eat/making-healthy-foodchoices/fats-and-diabetes.html.

American Diabetes Association. (2015c). What We Recommend; types of activity. Retrieved May 19, 2015, from http://www.diabetes.org/food-and-fitness/fitness/types-of-activity/whatwe-recommend.html.

American Diabetes Association. (2016). Physical Activity is Important. Retrieved December 27, 2016, from http://www.diabetes.org/food-and-fitness/fitness/physical-activityis-important.html.

American Diabetes Association. (2017). Standards of medical care in diabetes abridged for primary care providers. Clinical Diabetes, 35(1), 5-26. 
American Diabetes Association. (2018). Glycaemic targets: standards of medical care in diabetes. Diabetes Care, 41(Supplement 1), S55-S64.doi: 10.2337/dc18-S006.

Antoine, S., Pieper, D., Mathes, T., \& Eikermann, M. (2014). Improving the adherence of type 2 diabetes mellitus patients with pharmacy care: A systematic review of randomized controlled trials. BMC Endocrine Disorders, 14(1).doi:10.1186/1472-6823-14-53.

Arifulla, M., Lisha Jenny, J., Sreedharan, J., Muttappallymyalil, J., \& Basha, S. (2014). Patients' adherence to anti-diabetic medications in a hospital at Ajman, UAE. The Malaysian journal of medical sciences: MJMS, 21(1), 44.

Armstrong, D., Gosling, A., Weinman, J., \& Marteau, T. (1997). The Place of Inter-Rater Reliability in Qualitative Research: An Empirical Study. Sociology, 31(3), 597606.doi:10.1177/0038038597031003015.

Asdaq, S. (2018). Knowledge, Attitude, and Practice Regarding Diabetes Mellitus among General Public and Diabetic Patients in Riyadh, Saudi Arabia. Asian J Pharm, 12(01).268-276.

Awad, H., Al-Zubi, Z., \& Abdallah, A. (2016). A Quantitative Analysis of the Causes of Drug Shortages in Jordan: A Supply Chain Perspective. International Business Research, 9(6), 53. doi:10.5539/ibr.v9n6p53.

Awad, N. E., Seida, A. A., Shaffie, Z. E., El-Aziz, A. M. A., \& Awad, N. E. (2012). Hypoglycaemic activity of Artemisia herba-alba (Asso.) used in Egyptian traditional medicine as hypoglycaemic remedy. Journal of Applied Pharmaceutical Science, 2(03), 30-39.

Awwad, O., Akour, A., Al-Muhaissen, S., \& Morisky, D. (2015). The influence of patients' knowledge on adherence to their chronic medications: A cross-sectional study in Jordan. International Journal of Clinical Pharmacy, 37(3), 504-510.doi:10.1007/s11096-0150086-3.

Azaizeh, H., Fulder, S., Khalil, K., \& Said, O. (2003). Ethnobotanical knowledge of local Arab practitioners in the Middle Eastern region. Fitoterapia, 74(1-2), 98-108.doi:10.1016/s0367326x (02)00285.

Badran, M., \& Laher, I. (2012). Type II Diabetes Mellitus in Arabic-Speaking Countries. International Journal of Endocrinology, 2012, 1-11. doi:10.1155/2012/902873. 
Baig, A., Benitez, A., Quinn, M., \& Burnet, D. (2015). Family interventions to improve diabetes outcomes for adults. Annals of the New York Academy of Sciences, 1353(1), 89-112. doi:10.1111/nyas.1284.

Bailey, C., \& Day, C. (2004). Metformin: Its botanical background. Practical Diabetes International, 21(3), 115-117. doi:10.1002/pdi.606.

Bakhotmah, B., \& Alzahrani, H. (2010). Self-reported use of complementary and alternative medicine (CAM) products in topical treatment of diabetic foot disorders by diabetic patients in Jeddah, Western Saudi Arabia. BMC Research Notes, 3(1), 254.doi:10.1186/1756-0500-3-25.

Bakkar, M., Haddad, M., \& Gammoh, Y. (2017). Awareness of diabetic retinopathy among patients with type 2 diabetes mellitus in Jordan. Diabetes, metabolic syndrome and obesity: targets and therapy, 10, 435-441.

Bakri, F., Allan, A., Khader, Y., Younes, N., \& Ajlouni, K. (2012). Prevalence of Diabetic Foot Ulcer and its Associated Risk Factors among Diabetic Patients in Jordan. Jordan Medical Journal, 46(2), 118-125.

Bandura, A. (2001). Social cognitive theory: An agentic perspective. Annual review of psychology, 52(1), 1-26.

Barghouti, F., Abu-Rmaileh, N., Jallad, D., \& Abd-Qudah, Y. (2015). Leisure Time Physical Activity in Jordan: Knowledge and Sociodemographic Determinants. International Medical Journal, 22(4).

Barnard, N., Levin, S., Gloede, L., \& Flores, R. (2018). Turning the Waiting Room into a Classroom: Weekly Classes Using a Vegan or a Portion-Controlled Eating Plan Improve Diabetes Control in a Randomized Translational Study. Journal of the Academy of Nutrition and Dietetics, 118(6), 1072-1079. doi:10.1016/j.jand.2017.11.017.

Basheti, I., Hait, S., Qunaibi, E., Aburuz, S., \& Bulatova, N. (2016). Associations between patient factors and medication adherence: A Jordanian experience. Pharmacy Practice, 14(1), 639-639. doi:10.18549/pharmpract.2016.01.639.

Bauman, A., Bellew, B., Owen, N., \& Vita, P. (2001). Impact of an Australian mass media campaign targeting physical activity in 1998. American Journal of Preventive Medicine, 21(1), 41-47.doi:10.1016/s0749-3797(01)00313-0. 
Bawadi, H., Abu-Jamous, D., \& Tayyem, R. (2014). Evaluation of the dietary pattern of patients with type 2 diabetes in Northern Jordan: An inconvenient truth. International Journal of Diabetes in Developing Countries, 34(3), 134-138.

Bawadi, H., Al-Hamdan, Z., Bawadi, H., Ershidat, O., Hammad, F., \& Agraib, L. (2012). Cultural Eating Practices among Jordanians. Food and Nutrition Sciences, 03(06), 790-795. doi:10.4236/fns.2012.36106.

Bayat, F., Shojaeezadeh, D., Baikpour, M., Heshmat, R., Baikpour, M., \& Hosseini, M. (2013). The effects of education based on extended health belief model in type 2 diabetic patients: A randomized controlled trial. Journal of Diabetes \& Metabolic Disorders, 12(1), 45.doi:10.1186/2251-6581-12-45.

Becker, M., Maiman, L., Kirscht, J., Haefner, D., \& Drachman, R. (1978). The Health Belief Model and Prediction of Dietary Compliance: A Field Experiment. Journal of Health and Social Behaviour, 18(4), 348.doi:10.2307/2955344.

Bernard, H. (2000). Social research methods ( $\left.1^{\text {st }} e d.\right)$. Thousand Oaks, CA: Sage.

Bertalanffy, V. (1968). General system theory. New York, 41973(1968), 40.

Besen, D., Günüşen, N., Sürücü, H., \& Koşar, C. (2016). Predictor effect of Locus of Control (LOC) on self-care activities and metabolic control in individuals with type 2 diabetes. PeerJ, 4, e2722.doi: 10.7717/peerj.2722.

Bogdan, R., \& Biklen, S. (2003). Qualitative Research for Education: An introduction to Theories and Methods ( ${ }^{\text {th }}$ ed.). New York: Pearson Education group.

Bone, A. (2013). Optimising safety. Principles and Practice of Phytotherapy, 100-117. doi:10.1016/B978-0-443-06992-5.00005-0.

Boutayeb, A., Lamlili, M., Boutayeb, W., Maamri, A., Ziyyat, A., \& Ramdani, N. (2012). The rise of diabetes prevalence in the Arab region. Open Journal of Epidemiology, 02(02), 55-60. doi:10.4236/ojepi.2012.22009.

Bowling, A. (2014). Research methods in health: Investigating health and health services $\left(4^{\text {th }}\right.$ ed.). Maidenhead, Berkshire, England: Open University Press. 
Bowen, G. (2009). Supporting a grounded theory with an audit trail: An illustration. International Journal of Social Research Methodology, 12(4), 305316.doi:10.1080/13645570802156196.

Braun, V., \& Clarke, V. (2006). Using thematic analysis in psychology. Qualitative Research in Psychology, 3(2), 77-101.doi:10.1191/1478088706qp063oa

Bronfenbrenner, U. (1977). Toward an experimental ecology of human development. American Psychologist, 32(7), 513-531. doi:10.1037//0003-066x.32.7.513.

Bryman, A. (2012). Social research methods (4 ${ }^{\text {th }}$ ed.). Oxford: Oxford University Press.

Bryman, A. (2016). Social research methods (5 ${ }^{\text {th }}$ ed.). Oxford: Oxford University Press.

Carey, M. (1994). The group effect in focus groups: planning, implementation, and interpreting focus group research, in Critical issues in qualitative research methods / edited by Janice M. Morse. Sage, Thousand Oaks, CA, pp. 225-241.

Cohen, L., Manion, L., \& Morrison, K. (2011). Research methods in education ( ${ }^{\text {th }}$ ed.). London: Routledge.

Cowie, L., Morgan, M., White, P., \& Gulliford, M. (2009). Experience of continuity of care of patients with multiple long-term conditions in England. Journal of Health Services Research \& Policy, 14(2), 82-87. doi:10.1258/jhsrp.2009.008111.

Creswell, J. (2007). Qualitative inquiry and research design: Choosing among five traditions. London: Sage publications.

Creswell, J. (2014). Research design: Qualitative, quantitative, and mixed methods approaches ( $4^{\text {th }}$ ed.). Los Angeles: Sage publications.

Critical Appraisal Skills Programme. (2018). CASP Appraisal Checklists. Retrieved January 25, 2018, from: https://casp-uk.net/casp-tools-checklists.

Daoud, N., Osman, A., Hart, T., Berry, E., \& Adler, B. (2015). Self-care management among patients with type 2 diabetes in East Jerusalem. Health Education Journal, 74(5), 603-615.doi: $10.1177 / 0017896914555038$

Darawad, M., Mosleh, S., Khalil, A., Maharmeh, M., Hamdan-Mansour, A., \& Samarkandi, O. (2016). Investigating Physical Exercise among Jordanians with Diabetes Mellitus. Health, 08(02), 181-189. doi:10.4236/health.2016.82021. 
Davies, C. (2008). Reflexive ethnography: A guide to researching selves and others / Charlotte Aull Davies. (2 ${ }^{\text {nd }}$ ed.). London: Routledge.

DeHaven, M., Hunter, I., Wilder, L., Walton, J., \& Berry, J. (2004). Health programs in faithbased organizations: are they effective? American Journal of Public Health, 94(6), 1030-1036.

Dehghani-Tafti, A., Mahmoodabad, S., Morowatisharifabad, M., Ardakani, M., Rezaeipandari, H., \& Lotfi, M. (2015). Determinants of Self-Care in Diabetic Patients Based on Health Belief Model. Global Journal of Health Science, 7(5). doi:10.5539/gjhs.v7n5p33.

Denzin, N., \& Lincoln, Y. (1994). Handbook of qualitative research ( $1^{\text {st }}$ ed.). London: Sage.

Denzin, N., \& Lincoln, Y. (2005). Handbook of qualitative research ( $3^{\text {rd }}$ ed.). London: Sage.

Department of Statistics. (2017). Health Tables. Retrieved December 20, 2017, from http://dosweb.dos.gov.jo/products/statistical_yearbook2017.

Department of statistics. (2018). Estimated Population of the Kingdom by Governorate and Sex, at End-year 2017. October 1, 2018, from

http://dosweb.dos.gov.jo/DataBank/PopulationEstimares/2017/PopulationEstimates.pdf.

Diabetes UK. (2013). Self-monitoring of blood glucose (SMBG) for adults with Type 2 diabetes. London: Diabetes UK.

Diabetes UK. (2014). Diabetes Specialist Nurses: Improving Patient Outcomes and Reducing Costs. London: Diabetes UK.

Diabetes UK. (2017a). Diabetes Prevalence 2017. Retrieved December 19, 2017, from https://www.diabetes.org.uk/professionals/position-statements-reports/statistics/diabetesprevalence- 2017

Diabetes UK. (2017b). What is HbAlc? Retrieved May 19, 2017, from https://www.diabetes.org.uk/guide-to-diabetes/managing-your-diabetes/hbalc.

Diabetes UK. (2018a). Community Champions. Retrieved January 19, 2018, from https://www.diabetes.org.uk/get_involved/volunteer/community-champions.

Diabetes UK. (2018b). Diabetes Myths and frequently asked questions. Retrieved March 19, 2018, from https://www.diabetes.org.uk/diabetes-the-basics/myths-and-faqs. 
Diabetes UK. (2018c). Herbal and Natural Therapies. Retrieved February 9, 2018, from https://www.diabetes.co.uk/Diabetes-herbal.html.

Diabetes UK. (2018d). Is there a cure for diabetes? Retrieved February 19, 2018, from https://www.diabetes.org.uk/diabetes-the-basics/is-there-a-cure.

Diabetes UK. (2018e). What is a healthy, balanced diet for diabetes?. Retrieved January 5, 2018, from https://www.diabetes.org.uk/guide-to-diabetes/enjoy-food/eating-withdiabetes/what-is-a-healthy-balanced-diet.

Diabetes Wales. (2018). Diabetes Research Unit. Retrieved October 29, 2018, from http://www.diabeteswales.org.uk/en.

DiClemente, R., Crosby, R., \& Kegler, M. (2009). Emerging theories in health promotion practice and research ( $2^{\text {nd }}$ ed.). San Francisco: Jossey-Bass.

Draznin, B. (2016). Managing diabetes and hyperglycaemia in the hospital setting: A clinician's guide ( $1^{\text {st }}$ ed.). Alexandria: American Diabetes Association.

Dubois, C., Damour, D., Tchouaket, E., Clarke, S., Rivard, M., \& Blais, R. (2013). Associations of patient safety outcomes with models of nursing care organization at unit level in hospitals. International Journal for Quality in Health Care, 25(2), 110-117. doi:10.1093/intqhe/mzt019.

Dwyer, S., \& Buckle, J. (2009). The Space Between: On Being an Insider-Outsider in Qualitative Research. International Journal of Qualitative Methods, 8(1), 5463.doi:10.1177/160940690900800105.

El-Qudah, J. (2016). Dietary Knowledge among Female Diabetic Patients in Amman, Jordan. Current Research in Nutrition and Food Science Journal,4(2), 107-113.doi: 10.12944/CRNFSJ.4.2.04.

Egede, L., \& Ellis, C. (2009). Development and Psychometric Properties of the 12-Item Diabetes Fatalism Scale. Journal of General Internal Medicine, 25(1), 6166.doi:10.1007/s11606-009-1168-5.

Fisher, E., Brownson, C., O’Toole, M., Shetty, G., Anwuri, V., \& Glasgow, R. (2005). Ecological approaches to self-management: the case of diabetes. American Journal of Public Health, 95(9), 1523-1535. 
Fitzgerald, J., Stansfield, R., Tang, T., Oh, M., Frohna, A., Armbruster, B., \& Anderson, R. (2008). Patient and provider perceptions of diabetes: Measuring and evaluating differences. Patient Education and Counselling, 70(1), 118-125.

doi:10.1016/j.pec.2007.09.005.

Food and Drug Administration. (2013). Guidance for Industry, Tablet Scoring:

Nomenclature, Labelling, and Data for Evaluation. Retrieved October 19, 2018 from https://www.fda.gov/downloads/drugs/guidances/ucm269921.pdf.

Freeman, G., \& Hughes, J. (2010). Continuity of care and the patient experience. London: Kings Fund.

Gallagher, H., Gebhard, M., Nash, W., Occhipinti, N., \& Walke, B. (2008). The Jordanian Diabetes Crisis. Michigan: University of Michigan.

Gazmararian, J., Ziemer, D., \& Barnes, C. (2009). Perception of Barriers to Self-care Management among Diabetic Patients. The Diabetes Educator, 35(5), 778788.doi:10.1177/0145721709338527.

Giddens, A., \& Griffiths, S. (2006). Sociology (5 $5^{\text {th }}$ ed.) Cambridge: Polity Press.

Gill, P., Stewart, K., Treasure, E., \& Chadwick, B. (2008). Methods of data collection in qualitative research: interviews and focus groups. British dental journal, 204(6), 291.

Glanz, K. (2002). Health behaviour and health education: Theory, research, and practice ( $3^{\text {rd }}$ ed.). San Francisco: Jossey-Bass.

Gold, R. L. (1958) Roles in sociological field observation. Social Forces, 36, 217-223.

Golden, S., \& Earp, J. (2012). Social Ecological Approaches to Individuals and Their Contexts. Health Education \& Behaviour, 39(3), 364-372.doi:10.1177/1090198111418634.

Gopalan, A., Moss, H., Tao, Y., Zhu, J., \& Volpp, K. (2014). Patient Perceptions of Current Disease Control in Poorly Controlled Diabetes. Health, 06(15), 1964-1971. doi:10.4236/health.2014.615230.

Guest, G., Bunce, A., \& Johnson, L. (2006). How Many Interviews Are Enough? Field Methods, 18(1), 59-82.doi: 10.1177/1525822x05279903.

Guillemin, M., \& Gillam, L. (2004). Ethics, reflexivity, and "ethically important moments" in research. Qualitative inquiry, 10(2), 261-280.doi: 10.1177/1077800403262360. 
Habashneh, R., Khader, Y., Hammad, M. \& Almuradi, M. (2010). Knowledge and awareness about diabetes and periodontal health among Jordanians. Journal of Diabetes and Its Complications, 24(6), 409-414. doi:10.1016/j.jdiacomp.2009.06.001.

Hammersley, M. (1992). What is wrong with ethnography? (1 $1^{\text {st }}$ ed.). London: Routledge.

Hammersley, M., \& Atkinson, P. (1995). Ethnography: Principles in practice (2 ${ }^{\text {nd }}$ ed.) London: Routledge.

Hammersley, M., \& Atkinson, P. (2007). Ethnography: Principles in practice (3 ${ }^{\text {rd }}$ ed.). London: Routledge.

Helman, C. (2007). Culture, Health and Illness: An Introduction for Health Professionals $\left(5^{\text {th }}\right.$ ed.). Oxford: Elsevier Science.

Higher Council for Science and Technology. (2010). Defining Scientific Research Priorities in Jordan for the Years 2011-2020. Retrieved May 19, 2017 from http://www.hcst.gov.jo/sites/default/files/defining_scientific_research_priorities_in.pdf.

Hill, R., Fishbein, M., \& Ajzen, I. (1977). Belief, Attitude, Intention and Behaviour: An Introduction to Theory and Research. Contemporary Sociology, 6(2), 244.doi: $10.2307 / 2065853$.

Hillson, R. (2015). Diabetes care: A practical manual (2 ${ }^{\text {nd }}$ ed.). Oxford: Oxford University Press.

Holmes-Truscott, E., Blackberry, I., O’Neal, D., Furler, J., \& Speight, J. (2016). Willingness to initiate insulin among adults with type 2 diabetes in Australian primary care: Results from the Stepping up Study. Diabetes Research and Clinical Practice, 114, 126-135. doi:10.1016/j.diabres.2015.12.011.

Harmon, B. E., Kim, S. H., Blake, C. E., \& Hebert, J. R. (2014). Health care information in African-American churches. Journal of health care for the poor and underserved, 25(1), 242. doi:10.1353/hpu.2014.0047.

Hassan, H., Tohid, H., Amin, R., Bidin, M., Muthupalaniappen, L., \& Omar, K. (2013). Factors influencing insulin acceptance among type 2 diabetes mellitus patients in a primary care clinic: A qualitative exploration. BMC Family Practice, 14(1).doi: 10.1186/1471-2296-14-164. 
Huff, R., Kline, M., Peterson, D., \& Green, L. (2015). Health promotion in multicultural populations: A handbook for practitioners and students ( $3^{\text {rd }}$ ed.). Los Angeles: Sage.

IDF. (2012). Global Guideline for Type 2 Diabetes. Retrieved January 16, 2016, from http://www.diabetesatlas.org.

IDF. (2013). IDF Diabetes Atlas (6 ${ }^{\text {th }}$ ed.). Retrieved October 14, 2017, from http://www.diabetesatlas.org.

IDF. (2017). IDF Diabetes Atlas ( $8^{\text {th }}$ ed.). Retrieved August 4, 2018, from http://www.diabetesatlas.org.

Ilic, D., \& Rowe, N. (2013). What is the evidence that poster presentations are effective in promoting knowledge transfer? A state of the art review. Health Information \& Libraries Journal, 30(1), 4-12. doi:10.1111/hir.12015.

Institute of Medicine. (2003). Who Will Keep the Public Healthy? Educating Public Health Professionals for the 21st Century. Washington: The National Academies Press.

Irshaid, F. (2014). Prevalence of insulin- treated Type-2 diabetes mellitus in northern Jordan: Lifestyle, familial inheritance and maternal influence. European Scientific Journal, ESJ, 10(12).doi: 10.19044/esj.2014.v10n12p\%25p.

Jammal, H., Khader, Y., Alkhatib, S., Abujbara, M., Alomari, M., \& Ajlouni, K. (2013). Diabetic retinopathy in patients with newly diagnosed type 2 diabetes mellitus in Jordan: Prevalence and associated factors. Journal of Diabetes, 5(2), 172-179. doi:10.1111/17530407.12015 .

Jarab, A., Mukattash, T., Al-Azayzih, A., \& Khdour, M. (2018). A focus group study of patient's perspective and experiences of type 2 diabetes and its management in Jordan. Saudi Pharmaceutical Journal, 26(3), 301-305. doi:10.1016/j.jsps.2018.01.013.

Jeragh-Alhaddad, F., Waheedi, M., Barber, N., \& Brock, T. (2015). Barriers to medication taking among Kuwaiti patients with type 2 diabetes: A qualitative study. Patient Preference and Adherence, 9, 1491-503. doi:10.2147/ppa.s86719.

Jordanian Society for the Care of Diabetes. (2018). Events. Retrieved February 2, 2018, from www.jscd.jo. 
Kawafha, M. (2016). Effectiveness of Community Nurse Home Visits in Improvement of Infant Mortality and Health from the Perspectives of Community Health Nurses in Jordan. Gazi Medical Journal, 27(3), 118-123. doi:10.12996/gmj.2016.36.

Kelly, M., \& Huddy, C. (1999). Keeping your temple clean: Health promotion and religious function. Journal of Religion and Health, 38(4), 333-340.

Kenkre, J. (1997). The Role of the Nurse in Primary Care Research. In Y. Carter \& C. Thomas (Eds.). Research Methods in Primary Care (pp. 97-106). Oxford: Radcliffe Press.

Khader, Y., Khatatbeh, M., El-Salem, K., Amarin, Z., \& Bateiha, A. (2008). The Metabolic Syndrome -Among Patients Undergoing Cardiac Catheterization in Jordan. Journal of the CardioMetabolic Syndrome, 3(4), 224-228. doi:10.1111/j.1559-4572.2008.00020.

Khatib, F., Jarrah, N., Shegem, N., Bateiha, A., Abu-Ali, R., \& Ajlouni, K. (2006). Sexual dysfunction among Jordanian men with diabetes. Saudi Medical Journal, 27(3), 351-356.

Khatib, M., Efrat, S., \& Deeb, D. (2007). Knowledge, Beliefs, and Economic Barriers to Healthcare. Journal of Ambulatory Care Management, 30(1), 79-85.doi:10.1097/00004479200701000-0001.

Khattab, M., Khader, Y., Al-Khawaldeh, A., \& Ajlouni, K. (2010). Factors associated with poor glycaemic control among patients with Type 2 diabetes. Journal of Diabetes and Its Complications, 24(2), 84-89.doi: 10.1016/j.jdiacomp.2008.12.008.

Khatun, H., Rahman, A., Biswas, M., \& Islam, A. U. (2011). Water-soluble Fraction of Abelmoschus esculentus L Interacts with Glucose and Metformin Hydrochloride and Alters Their Absorption Kinetics after Coadministration in Rats. ISRN Pharmaceutics, 2011, 1-5. doi:10.5402/2011/260537.

Khawaja, N., Abu-Shennar, J., Saleh, M., Dahbour, S., Khader, Y., \& Ajlouni, K. (2018). The prevalence and risk factors of peripheral neuropathy among patients with type 2 diabetes mellitus; the case of Jordan. Diabetology \& Metabolic Syndrome, 10(1), 110.doi:10.1186/s13098-018-0309-6

Kitzinger, J. (1994). The methodology of Focus Groups: The importance of interaction between research participants. Sociology of Health and Illness, 16(1), 103-121. doi:10.1111/14679566.ep11347023.

Krueger, R., \& Casey, M. (2002). Designing and conducting focus group interviews. 
Ley, P. (1982). Satisfaction, compliance and communication. British Journal of Clinical Psychology, 21(4), 241-254. doi:10.1111/j.2044-8260.1982.tb00562.

Lincoln, Y., \& Guba, E. (1985). Naturalistic inquiry (1 ${ }^{\text {st }}$ ed.). London: Sage.

Mabry, R., Al-Busaidi, Z., Reeves, M., Owen, N., \& Eakin, E. (2014). Addressing physical inactivity in Omani adults: Perceptions of public health managers. Public Health Nutrition, 17(03), 674-681.doi:10.1017/s1368980012005678.

Madanat, H., Troutman, K., \& Al-Madi, B. (2008). The nutrition transition in Jordan: The political, economic and food consumption contexts. Promotion \& Education, 15(1), 6-10.doi: $10.1177 / 1025382307088092$.

Maps.com. (2011). Jordan physical map. Retrieved October 2, 2018, from http://www.maps.com/ref_map.aspx?pid=12108.

Marcus, B., Owen, N., Forsyth, L., Cavill, N., \& Fridinger, F. (1998). Physical activity interventions using mass media, print media, and information technology. American Journal of Preventive Medicine, 15(4), 362-378.doi: 10.1016/s0749-3797(98)00079-8.

Mason, J. (2002). Qualitative researching (2 ${ }^{\text {nd }}$ ed.). London: Sage.

Mauss, M. (2002). The gift: The form and reason for exchange in archaic societies. London: Routledge.

Mayberry, L., \& Osborn, C. (2012). Family Support, Medication Adherence, and Glycaemic Control among Adults with Type 2 Diabetes. Diabetes Care, 35(6), 1239-1245.doi: $10.2337 / \mathrm{dc} 11-2103$.

Mcleroy, K. R., Bibeau, D., Steckler, A., \& Glanz, K. (1988). An Ecological Perspective on Health Promotion Programs. Health Education Quarterly, 15(4), 351-377.doi: $10.1177 / 109019818801500401$.

McLeroy, K., Norton, B., Kegler, M., Burdine, J., \& Sumaya, C. (2003). Community-based interventions. American Journal of Public Health 93 (4), 529-533.

Mele, C., Pels, J., \& Polese, F. (2010). A Brief Review of Systems Theories and Their Managerial Applications. Service Science, 2(1-2), 126-135. doi:10.1287/serv.2.1_2.126.

Menzies, I. (1960). A Case-Study in the Functioning of Social Systems as a Defence against Anxiety. Human Relations, 13(2), 95-121.doi: 10.1177/001872676001300201. 
Migdadi, E., Courtenay, A., Tekko, I., Mccrudden, M., Kearney, M., Mcalister, E., Donnelly, R. (2018). Hydrogel-forming microneedles enhance transdermal delivery of metformin hydrochloride. Journal of Controlled Release, 285, 142-151. doi:10.1016/j.jconrel.2018.07.009.

MOH, \& Arbaji, A. (2005). Evaluation of Utilization of Health Services Delivery and Health Status; Primary Health Care Initiatives. Amman, Jordan.

MOH. (2009). Initial report for Primary Health care services in Jordan. Retrieved May 25, 2016, from http://www.csb.gov.jo/csb/Researchers/CSBReleases/studies/ PDF.

MOH. (2012).The national strategy and plan of action against diabetes, hypertension, dyslipidaemia and obesity in Jordan. Amman: Ministry of Health.

MOH. (2016). The Guide to Health Insurance System and its Amendments. Retrieved December 25, 2016, from http://www.hia.gov.jo/Docs/Systems/InsuranceSystem_2016.pdf

MOH. (2018a). Health Sector Guide. Retrieved June 15, 2018, from http://www.moh.gov.jo/Pages/viewpage.aspx?pageID=378

MOH. (2018b). Primary Health Care Services. Retrieved August 10, 2018, from http://www.moh.gov.jo/Pages/viewpage.aspx?pageID=233.

Morgan, D., \& Krueger, R. (1998). The focus group kit. London: Sage.

Morse, J. M. and Field, P. A. (1996) Nursing research: the application of qualitative approaches ( $2^{\text {nd }}$ ed.). London: Chapman \& Hall.

Micro Clinic International. (2017). How it works. Retrieved December 1, 2017, from https://microclinics.org/how-it-works.

Muecke, M. (1994). On the evaluation of ethnographies. In Morse, J., Critical issues in qualitative research methods (pp. 187-209). London: Sage.

Musaiger, A., Al-Mannai, M., Tayyem, R., Al-Lalla, O., Ali, E., Kalam, F., Chirane, M. (2012). Prevalence of Overweight and Obesity among Adolescents in Seven Arab Countries: A CrossCultural Study. Journal of Obesity, 2012, 1-5. doi:10.1155/2012/981390.

Nabolsi, M., \& Carson, A. (2011). Spirituality, illness and personal responsibility: The experience of Jordanian Muslim men with coronary artery disease. Scandinavian Journal of Caring Sciences, 25(4), 716-724. doi:10.1111/j.1471-6712.2011.00882. 
Naidoo, J., \& Wills, J. (2009). Foundations of health promotion ( $3^{\text {rd }}$ ed.). Edinburgh: Elsevier. Naidoo, J., \& Wills, J. (2016). Foundations for health promotion $\left(4^{\text {th }}\right.$ ed.). Amsterdam: Elsevier.

National Centre for Diabetes, Endocrinology and Genetics. (2018). Retrieved March 10, 2018, from http://www.ncd.org.jo.

National Health Service UK. (2011). Splitting tablets may affect dosage. Retrieved April 12, 2016, from https://www.nhs.uk/news/medication/splitting-tablets-may-affect-dosage.

National Health Service UK. (2013). Guidance for the implementation of repeat dispensing. Retrieved September 12, 2018, from http://www.nhsemployers.org/ /media/Employers/Publications/repeat-dispensing-guide.pdf. National Health Service UK. (2014). Guideline for the Administration of Insulin by Nursing Staff. Retrieved May 19, 2014, from http://www.nhslanarkshire.org.uk/Services/Diabetes/Documents/Guideline for Administration of Insulin by Nursing Staff.pdf.

National Health Service UK. (2015). Healthy weight. Retrieved May 15, 2016, from https://www.nhs.uk/live-well/healthy-weight/\#limitations-of-the-bmi.

National Health Service UK. (2016a). Exercise for depression. Retrieved December 1, 2017, from https://www.nhs.uk/conditions/stress-anxiety-depression/exercise-for-depression.

National Health Service UK. (2016b). Side effects, statins. Retrieved June 10, 2018, from https://www.nhs.uk/conditions/statins/side-effects.

National Health Service UK. (2016c). Unintentional weight loss. Retrieved May 10, 2017, from

https://www.nhs.uk/conditions/unintentional-weight-loss.

National Institute for Health and Care Excellence. (2015). Diabetic foot problems: prevention and management. Retrieved June 1, 2016, from https://www.nice.org.uk/guidance/ng19.

Nazer, L., \& Tuffaha, H. (2017). Health Care and Pharmacy Practice in Jordan. The Canadian Journal of Hospital Pharmacy, 70(2), 150-155. doi:10.4212/cjhp.v70i2.1649.

Noble, H., \& Smith, J. (2015). Issues of validity and reliability in qualitative research. Evidence Based Nursing, 18(2), 34-35.doi: 10.1136/eb-2015-102054. 
Nuttall, F. (2015). Body mass index: obesity, BMI, and health: a critical review. Nutrition today, 50(3), 117.doi: 10.1097/NT.0000000000000092.

Obeidat, A., Ahmad, M., Haddad, F, \& Azzeh, F. (2015). Alarming high prevalence of metabolic syndrome among Jordanian adults. Pakistan Journal of Medical Sciences, 31(6). doi:10.12669/pjms.316.7714.

Odhayani, A., Tayel, S., \& Al-Madi, F. (2017). Foot care practices of diabetic patients in Saudi Arabia. Saudi Journal of Biological Sciences, 24(7), 1667-1671.

doi:10.1016/j.sjbs.2015.12.003.

O’Hea, E., Grothe, K., Bodenlos, J., Boudreaux, E., White, M., \& Brantley, P. (2005). Predicting Medical Regimen Adherence: The Interactions of Health Locus of Control Beliefs. Journal of Health Psychology, 10(5), 705-717.doi:10.1177/1359105305055330.

Otoom, S., Al-Safi, S., Kerem, Z., \& Alkofahi, A. (2006). The Use of Medicinal Herbs by Diabetic Jordanian Patients. Journal of Herbal Pharmacotherapy, 6(2), 31-41.doi: 10.1300/j157v06n02_03.

Ozcelik, F., Yiginer, O., Arslan, E., Serdar, M., Uz, O., Kardesoglu, E., \& Kurt, I. (2010). Association between glycaemic control and the level of knowledge and disease awareness in type 2 diabetic patients. Pol Arch Med Wewn, 120(10), 399-406.

Padela, A., Killawi, A., Heisler, M., Demonner, S., \& Fetters, M. (2010). The Role of Imams in American Muslim Health: Perspectives of Muslim Community Leaders in Southeast Michigan. Journal of Religion and Health, 50(2), 359-373.doi:10.1007/s10943-010-9428-6.

Parker, E., Baldwin, G., Israel, B., \& Salinas, M. (2004). Application of health promotion theories and models for environmental health. Health Education \& Behaviour, 31(4), 491-509. doi: 10.1177/1090198104265601.

Payyappallimana, U. (2010). Role of traditional medicine in primary health care: an overview of perspectives and challenging. Yokohama Journal of Social Sciences, 14(6), 721-743.

Permpongkosol, S. (2011). Iatrogenic disease in the elderly: Risk factors, consequences, and prevention. Clinical Interventions in Aging, 6, 77-82. doi:10.2147/cia.s10252.

Pearson, A., Vaughan, B., \& Fitzgerald, M. (2005). Nursing models for practice (3 ${ }^{\text {rd }}$ ed.). Edinburgh: Butterworth-Heinemann 
Peek, M., Ferguson, M., Roberson, T., \& Chin, M. (2014). Putting Theory Into Practice: A Case Study of Diabetes-Related Behavioural Change Interventions on Chicago's South Side. Health Promotion Practice, 15(2 suppl), 1-16.doi:10.1177/1524839914532292.

Pellatt, G. (2003). Ethnography and reflexivity: Emotions and feelings in fieldwork. Nurse Researcher, 10(3), 28-37. doi:10.7748/nr2003.04.10.3.28.c5894.

Pope, C., Ziebland, S., \& Mays, N. (2000). Analysing Qualitative Data. Qualitative Research in Health Care, 63-81.doi:10.1002/9780470750841.

Public Health England. (2018). Diabetes Prevention Week. Retrieved 28 October, 2018, from https://campaignresources.phe.gov.uk/resources/campaigns/72-diabetes-prevention-week

Purbafrani, A., Hashemi, G., Amirhosein, S., Bayyenat, S., Taghizade Moghaddam, H., \& Saeidi, M. (2014). The Benefits of Honey in Holy Quran. International Journal of Pediatrics, 2(3.3), 67-73.

Qidwai, W., Ashfaq, T., Khoja, T., \& Merchant, K. (2011). Equity in Healthcare: Status, Barriers, and Challenges. World Family Medicine Journal /Middle East Journal of Family Medicine, 9(6), 33-38. doi:10.5742/mejfm.2011.96035

Rabiu, M., Al Bdour, M., Abu Ameerh, M., \& Jadoon, M. (2015). Prevalence of blindness and diabetic retinopathy in northern Jordan. European journal of ophthalmology, 25(4), 320327.doi: 10.5301/ejo.5000557.

Richard, L., Gauvin, L., \& Raine, K. (2011). Ecological Models Revisited: Their Uses and Evolution in Health Promotion Over Two Decades. Annual Review of Public Health, 32(1), 307-326.doi:10.1146/annurev-publhealth-031210-101141.

Richards, T., Coulter, A., \& Wicks, P. (2015). Time to deliver patient centred care. Bmj, 350, 1-2. doi:10.1136/bmj.h530.

Rintala, T., Jaatinen, P., Paavilainen, E., \& Åstedt-Kurki, P. (2013). Interrelation between Adult Persons with Diabetes and Their Family. Journal of Family Nursing, 19(1), 328.doi:10.1177/1074840712471899.

Reeves, S., Kuper, A., \& Hodges, B. (2008). Qualitative research methodologies: ethnography. Bmj, 337, 1-2. doi:10.1136/bmj.a1020 
Rosen, C. Brown, J. Heiman, S. Leiblum, S. (2000). The Female Sexual Function Index (FSFI): A Multidimensional Self-Report Instrument for the Assessment of Female Sexual Function. Journal of Sex \& Marital Therapy, 26(2), 191-208.doi:10.1080/009262300278597.

Rosenstock, I. M. (1974). Historical Origins of the Health Belief Model. Health Education Monographs, 2(4), 328-335.doi:10.1177/109019817400200403.

Rosenstock, I., Strecher, V., \& Becker, M. (1988). Social Learning Theory and the Health Belief Model. Health Education Quarterly, 15(2), 175183.doi:10.1177/109019818801500203.

Rotter, J. B. (1954). Social learning and clinical psychology. New York: Johnson Reprint Corp.

Rotter, J. (1966). Generalized expectancies for internal versus external control of reinforcement. Psychological Monographs: General and Applied,80(1), 128.doi:10.1037/h0092976.

Royal Health Awareness Society. (2017). Healthy Schools National Accreditation. Retrieved July 11, 2018, from http://www.rhas.org.jo/Contents/Healthy_Schools_National_Accreditation.

Saad, B., Azaizeh, H., \& Said, O. (2005). Tradition and Perspectives of Arab Herbal Medicine: A Review. Evidence-Based Complementary and Alternative Medicine, 2(4), 475-479. doi:10.1093/ecam/neh133.

Saadia, Z., Rushdi, S., Alsheha, M., Saeed, H., \& Rajab, M. (2010). A study of knowledge attitude and practices of Saudi women towards diabetes mellitus. A (KAP) study in Al-Qassim region. The Internet Journal of Health, 11(2).

Sabbah, K., \& Alshehri, A. (2014). Practice and perception of self - management among diabetics in Taif, KSA: Impact of demographic factors. International Journal of Medical Science and Public Health, 3(3), 277. doi:10.5455/ijmsph.2013.141220132.

Salayta, W., \& Aleyadeh, A. (2010). The outcome of diabetic foot infection in Jordanian cohort. Rawal Medical Journal, 35(2), 214-216.

Sanz, M., Ceriello, A., Buysschaert, M., Chapple, I., Demmer, R., Graziani, F., Vegh, D. (2018). Scientific evidence on the links between periodontal diseases and diabetes: Consensus report and guidelines of the joint workshop on periodontal diseases and diabetes by the 
International diabetes Federation and the European Federation of Periodontology. Diabetes Research and Clinical Practice, 137, 231-241. doi:10.1016/j.diabres.2017.12.001.

Savage, J. (2000). Ethnography and health care. BMJ, 321(7273), 1400-1402.

Sayorwan, W. (2013). Effects of Inhaled Rosemary Oil on Subjective Feelings and Activities of the Nervous System. Scientia Pharmaceutica, 81(2), 531-542. doi:10.3797/scipharm.120905.

Schwandt, T., Lincoln, Y., \& Guba, E. (2007). Judging interpretations: But is it rigorous? Trustworthiness and authenticity in naturalistic evaluation. New Directions for Evaluation, 114, 11-25. doi:10.1002/ev.223.

Scott, J. (1990). A matter of record: Documentary sources in social research. Cambridge: Polity Press.

Seifarth, C., Schehler, B., \& Schneider, H. (2012). Effectiveness of Metformin on Weight Loss in Non-Diabetic Individuals with Obesity. Experimental and Clinical Endocrinology \& Diabetes, 121(01), 27-31.doi:10.1055/s-0032-1327734.

Shabibi, P., Zavareh, M., Sayehmiri, K., Qorbani, M., Safari, O., Rastegarimehr, B., \& Mansourian, M. (2017). Effect of educational intervention based on the Health Belief Model on promoting self-care behaviours of type-2 diabetes patients. Electronic Physician, 9(12), 5960-5968.doi: 10.19082/5960.

Shah, P., \& Mountain, D. (2007). The medical model is dead - long live the medical model. British Journal of Psychiatry, 191(05), 375-377. doi:10.1192/bjp.bp.107.037242.

Sharara, E., Akik, C., Ghattas, H., \& Obermeyer, C. (2018). Physical inactivity, gender and culture in Arab countries: A systematic assessment of the literature. BMC Public Health, 18(1), 1-19.doi:10.1186/s12889-018-5472-z.

Shuriquie, M., While, A., \& Fitzpatrick, J. (2008). Nursing work in Jordan: An example of nursing work in the Middle East. Journal of Clinical Nursing, 17(8), 999-1010. doi:10.1111/j.1365-2702.2007.01973.

Silverman, D. (2011). Interpreting qualitative data: A guide to the principles of qualitative research. ( $4^{\text {th }}$ ed.). Los Angeles: Sage. 
Singab, A., \& Youssef, F. (2014). Medicinal Plants with Potential Antidiabetic Activity and their Assessment. Medicinal \& Aromatic Plants, 03(01). doi:10.4172/2167-0412.1000151.

Skovlund, S., \& Peyrot, M. (2005). The Diabetes Attitudes, Wishes, and Needs (DAWN) program: A new approach to improving outcomes of diabetes care. Diabetes Spectrum, 18(3), 136-142.

Snelgrove, S. (2006). Factors contributing to poor concordance in health care. Medicine, 46(1), 119-29.

Steven, D., \& Ehrlich, N. (2009). Solutions Acupuncture, a private practice specializing in complementary and alternative medicine. Health, 2 (11).

Stokols, D., Pelletier, K., \& Fielding, J. (1996). The Ecology of Work and Health: Research and Policy Directions for the Promotion of Employee Health. Health Education Quarterly, 23(2), 137-158.doi:10.1177/109019819602300202.

Subhi, L., Kendall, P., Al-Shafaee, M., \& Al-Adawi, S. (2016). Health Beliefs of People with Type 2 Diabetes in Primary Health Care in Muscat, Oman: A Qualitative Approach. International Journal of Clinical Nutrition \& Dietetics, 2(1), 1-7. doi:10.15344/2456-8171/2016/106

Sukkarieh-Haraty, O., Egede, L., Kharma, J., \& Bassil, M. (2017). Predictors of Diabetes Fatalism among Arabs: A Cross-Sectional Study of Lebanese Adults with Type 2 Diabetes. Journal of Religion and Health, 57(3), 858-868.doi:10.1007/s10943-017-0430-0.

Sulaiman, N., Mahmoud, I., Hussein, A., Elbadawi, S., Abusnana, S., Zimmet, P., \& Shaw, J. (2018). Diabetes risk score in the United Arab Emirates: A screening tool for the early detection of type 2 diabetes mellitus. BMJ Open Diabetes Research \& Care, 6(1), 16.doi:10.1136/bmjdrc-2017-000489.

Takruri, H., \& Alkurd, R. (2014). Intakes of Fats, Cholesterol, and Micronutrients as Risk Factors for Cardiovascular Disease in Jordan. Jordan Journal of Biological Sciences, 7(2), 119-126.doi:10.12816/0008225.

Taylor, D., Bury, M., Campling, N., Carter, S., Garfield, S., Newbould, J., \& Rennie, T. (2006). A Review of the use of the Health Belief Model (HBM), the Theory of Reasoned Action (TRA), the Theory of Planned Behaviour (TPB) and the Trans-Theoretical Model (TTM) to study and 
predict health related behaviour change. London, UK: National Institute for Health and Clinical Excellence, 1-215.

The Higher Health Council. (2015). The National Strategy for Health Sector in Jordan 2015 2019. Retrieved September 15, 2016, from http://www.hhc.gov.jo/uploadedimages/20152019.pdf.

The University of Jordan. (2018). Department of Community Health Nursing. Retrieved September 1, 2018, from http://nursing.ju.edu.jo/Departments/CommunityHealthNursing.

Unger, J. (2012). Diabetes Management in Primary Care (2 $2^{\text {nd }}$ ed.). Philadelphia: Lippincott Williams and Wilkins.

United Nations Refugee Agency. (2018). Fact Sheet. Retrieved October 10, 2018, from https://reliefweb.int/sites/reliefweb.int/files/resources/FactSheetJordanFebru.pdf.

United Nations Relief and Works Agency. (2016). Facts \& Figures. Retrieved October 1, 2018, from https://www.unrwa.org/where-we-work/jordan.

Wakefield, M., Loken, B., \& Hornik, R. (2010). Use of mass media campaigns to change health behaviour. The Lancet, 376(9748), 1261-1271.doi:10.1016/s0140-6736(10)60809-4.

Wallace, C. \& Kenkre, J. (2015). Report: Consensus for the priorities for public health in Wales. Retrieved 20 October, 2018, from https://www.researchgate.net/publication/313599978_Consensus_for_the_Priorities_for_Publ ic_Health_in_Wales.

Wazaify, M., Afifi, F., El-Khateeb, M., \& Ajlouni, K. (2011). Complementary and alternative medicine use among Jordanian patients with diabetes. Complementary Therapies in Clinical Practice, 17(2), 71-75. doi:10.1016/j.ctcp.2011.02.002.

Wazaify, M., Alawwa, I., Yasein, N., Al-Saleh, A., \& Afifi, F. (2013). Complementary and alternative medicine (CAM) use among Jordanian patients with chronic diseases. Complementary Therapies in Clinical Practice, 19(3), 153-157. doi:10.1016/j.ctcp.2013.03.001.

Wilkinson, S. (1999). Focus groups: A feminist method. Psychology of women quarterly, 23(2), 221-244. 
Wills, J. (2014). Fundamentals of health promotion for nurses ( $2^{\text {nd }}$ ed.). Chichester: WileyBlackwell.

World Bank. (2016). The World Bank data, life expectancy at birth. Retrieved June 2, 2018, from https://data.worldbank.org/indicator/SP.DYN.LE00.IN.

World Health Organization. (1948). Constitution of the World Health Organisation. New York. Retrieved November 12, 2015, from http://apps.who.int/gb/bd/PDF/bd47/EN/constitutionen.pdf.

World Health Organization. (1978). Declaration of Alma-Ata International Conference on Primary Health Care, Alma-Ata, USSR. Retrieved July 2, 2016, from http://www.who.int/publications/almaata_declaration_en.pdf.

World Health Organization. (1996). Health promotion through Islamic lifestyles: the Amman Declaration. Alexandria: Egypt.

World Health Organization, \& Sabatâe, E. (2003). Adherence to Long-term Therapies: Evidence for Action. Geneva: World Health Organization.

World Health Organization. (2009a). Global health risks: mortality and burden of disease attributable to selected major risks. Retrieved August 2, 2017, from https://www.who.int/healthinfo/global_burden_disease/GlobalHealthRisks_report_full.pdf World Health Organization. (2009b). WHO guidelines on hand hygiene in health care. Geneva: World Health Organization.

World Health Organization. (2011). Global recommendations on physical activity for health. Geneva: World Health Organization.

World Health Organisation. (2014). WHO Traditional Medicine Strategy. Retrieved May 1, 2016, from http://apps.who.int/iris/bitstream/handle/10665/92455/9789241506090_eng.pdf.

World Health Organisation. (2015). Jordan: WHO statistical profile. Retrieved July 2, 2017, from http://www.who.int/gho/countries/jor/country_profiles/en.

World Health Organization. (2016a). Global report on diabetes. Geneva: World Health Organization.

World Health Organisation. (2016b). Jordan: Diabetes country profiles. Retrieved June 7, 2018, from https://www.who.int/diabetes/country-profiles/jor_en.pdf. 
World Health Organisation. (2018a). Health education. Retrieved March 2, 2018, from http://www.who.int/topics/health_education/en.

World Health Organisation. (2018b). Health promotion. Retrieved March 2, 2018, from http://www.who.int/topics/health_promotion/en.

World Health Organisation. (2018c). Healthy Settings. Retrieved April 12, 2018, from https://www.who.int/healthy_settings/about/en.

World Health Organisation. (2018d). Patient support groups. Retrieved May 12, 2018, from https://www.who.int/genomics/public/patientsupport/en.

X-Pert Health. (2018). X-PERT Health Services. Retrieved September 12, 2018, from https://www.xperthealth.org.uk.

Yacoub, M., Demeh, W., Darawad, M., Barr, J., Saleh, A., \& Saleh, M. (2014). An assessment of diabetes-related knowledge among registered nurses working in hospitals in Jordan. International Nursing Review, 61(2), 255-262. doi:10.1111/inr.12090.

Yasein, N., \& Masa'd, D. (2011). Metabolic syndrome in family practice in Jordan: a study of high-risk groups. Eastern Mediterranean Health Journal, 17(12), 943-948.

Zahran, Z. (2011). Nurse education in Jordan: History and development. International Nursing Review, 59(3), 380-386. doi:10.1111/j.1466-7657.2011.00947.

Zindah, M., Belbeisi, A., Walke, H., \& Mokdad, A. (2008). Obesity and diabetes in Jordan: findings from the Behavioural Risk Factors Surveillance System, 2004. Prev Chronic Dis, 5(1).

Zoughbie, D., Watson, K., Bui, N., Farraj, R., Prescott, M., \& Ding, E. (2014). Long-term bodyweight and glucose management effects of the Microclinic Social Network Health Behavioural Program in Amman, Jordan: 2-year results. The Lancet Global Health, 2, 1920.doi: 10.1016/S2214-109X(15)70041-0. 